Aus der Klinik für Kinder- und Jugendmedizin

(Prof. Dr. med. J. Gärtner)

der Medizinischen Fakultät der Universität Göttingen

\title{
Charakterisierung von positiven und negativen Prädiktoren für eine erfolgreiche \\ Adipositastherapie im Kindes- und Jugendalter nach Abschluss der Behandlung
}

\author{
INAUGURAL - DISSERTATION \\ zur Erlangung des Doktorgrades \\ der Medizinischen Fakultät der \\ Georg-August-Universität zu Göttingen
}

vorgelegt von

Lina Josr Sabih

aus Peine

Göttingen 2018 
Dekan:

I. Berichterstatter/in:

II. Berichterstatter/in:

III. Berichterstatter/in:

Tag der mündlichen Prüfung:
Prof. Dr. rer. nat. H. K. Kroemer

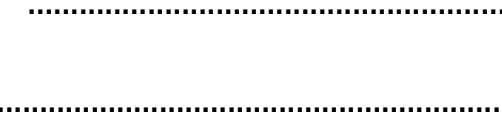


Hiermit erkläre ich, die Dissertation mit dem Titel "Charakterisierung von positiven und negativen Prädiktoren für eine erfolgreiche Adipositastherapie im Kindes- und Jugendalter nach Abschluss der Behandlung" eigenständig angefertigt und keine anderen als die von mir angegebenen Quellen und Hilfsmittel verwendet zu haben.

Göttingen, den

(Unterschrift) 


\section{Inhaltsverzeichnis}

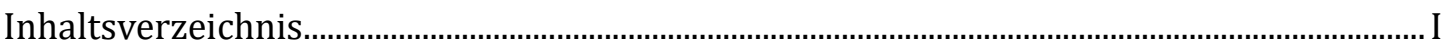

Abbildungsverzeichnis ............................................................................................................. III

Tabellenverzeichnis..............................................................................................................................

Abkürzungsverzeichnis ........................................................................................................... VII

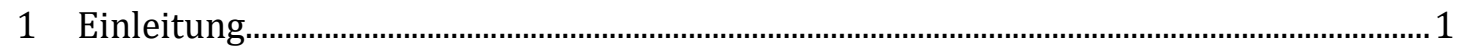

1.1 Definition von Adipositas im Kindes- und Jugendalter .............................................................. 1

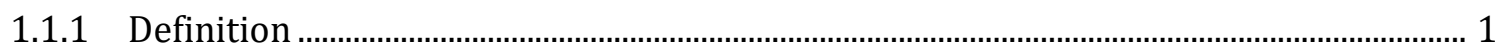

1.1.2 Methoden zur Bestimmung des Körperfettanteils .......................................................... 1

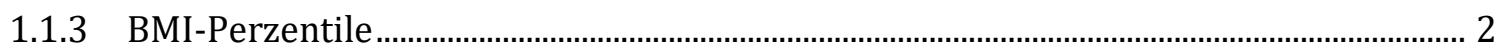

1.1.4 BMI-SDS

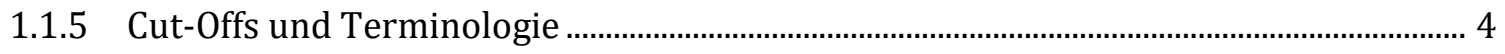

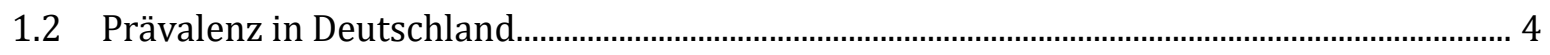

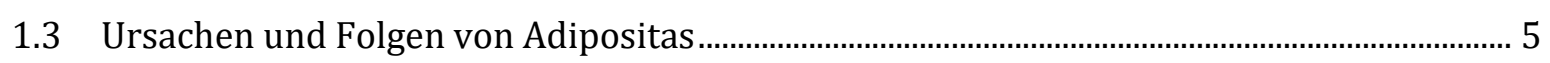

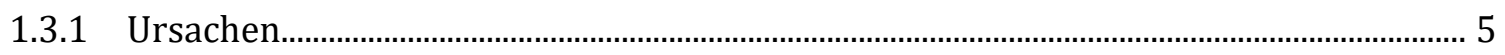

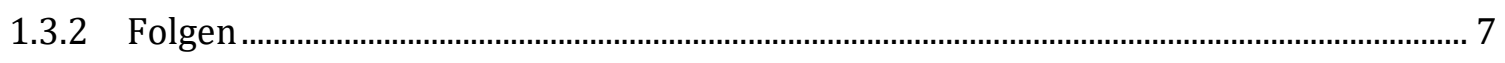

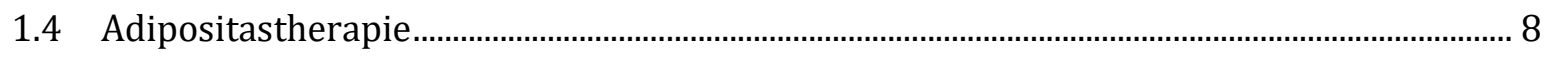

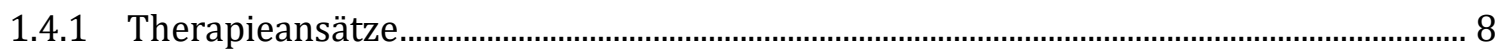

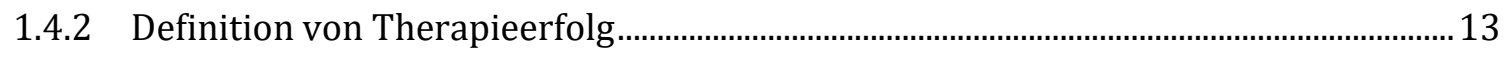

1.4.3 Aktuelle Therapieangebote .................................................................................................. 14

1.4.4 Erfolg und Langzeitverläufe von Therapieangeboten...................................................... 17

1.4.5 Prädiktoren für den Therapieerfolg ............................................................................... 19

1.5 Adipositas als chronische Erkrankung ………....................................................................... 21

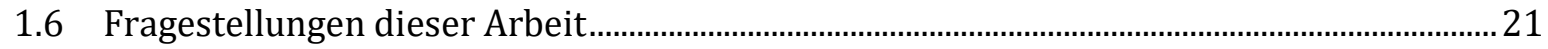

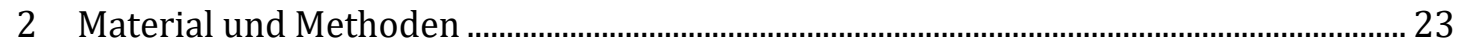

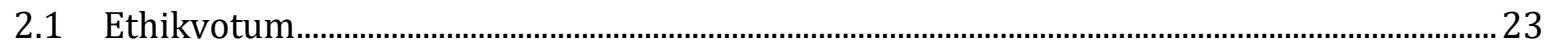

2.2 Patientenkollektiv und Studiendesign.................................................................................23

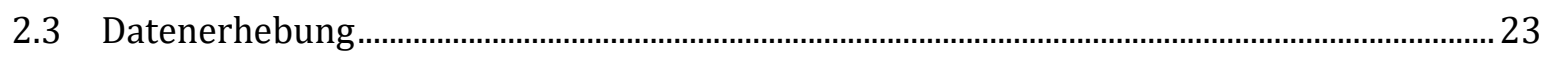

2.4 Methoden der Datenanalyse ..................................................................................................24

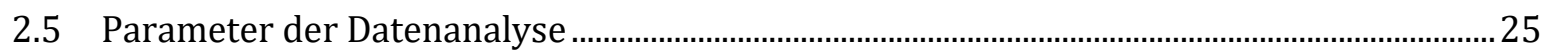

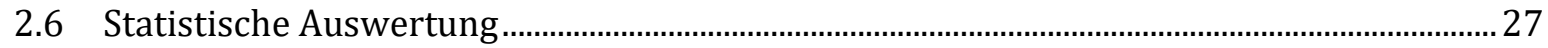

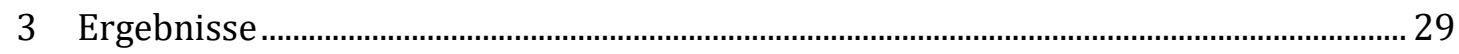

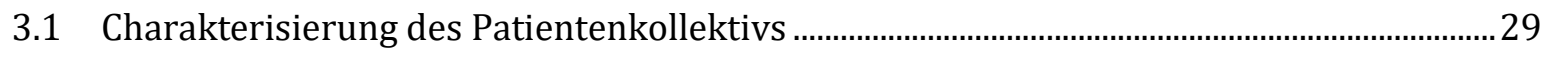

3.2 Therapieerfolg ......................................................................................................................

3.3 Untersuchung des Einflusses therapiebezogener Parameter auf die BMI-SDS-Reduktion 31

3.3.1 Therapie 


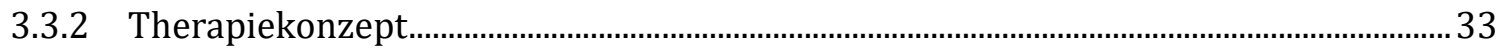

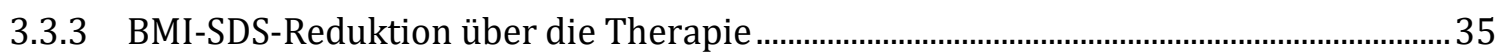

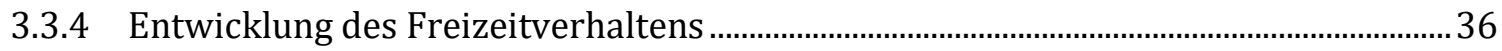

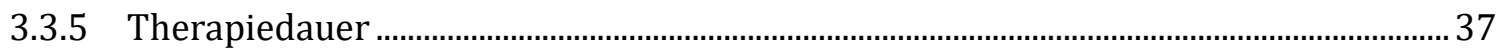

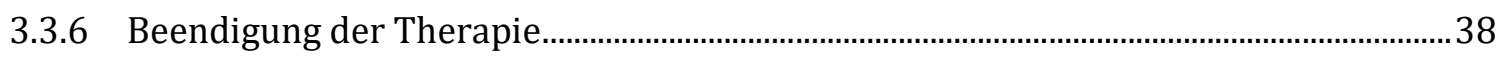

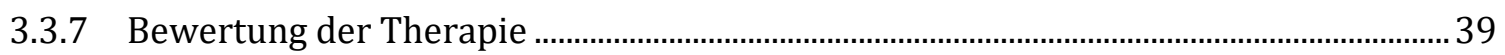

3.4 Untersuchung des Einflusses therapieunabhängiger Parameter auf die BMI-SDS-

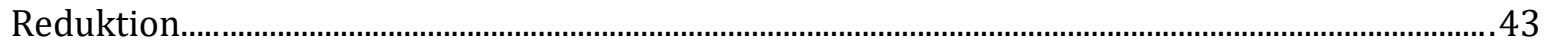

3.4.1 Motivation vor Therapiebeginn und Therapieziel............................................................. 43

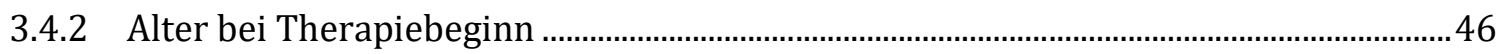

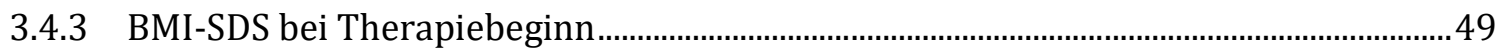

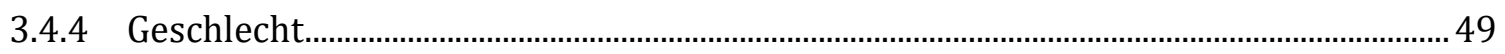

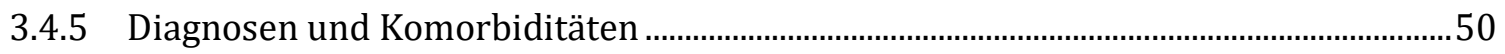

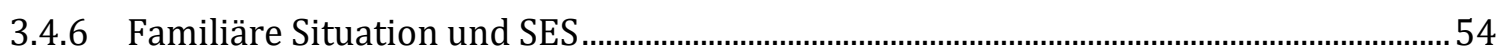

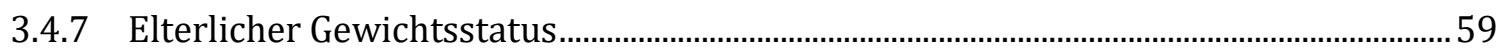

3.5 Untersuchung des Einflusses sonstiger Parameter auf die BMI-SDS-Reduktion................60

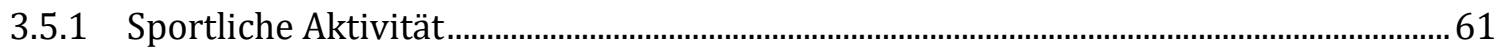

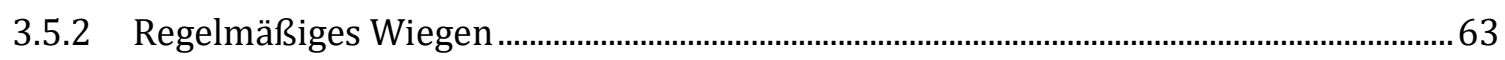

4 Diskussion 64

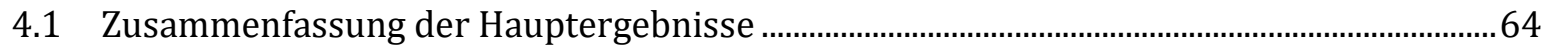

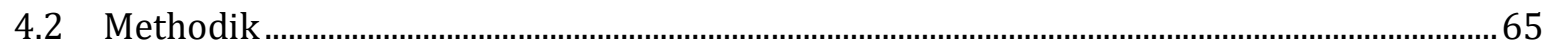

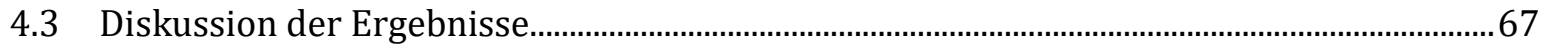

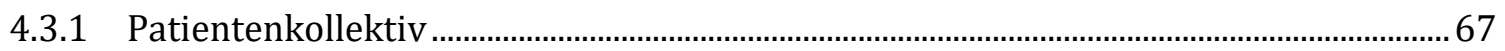

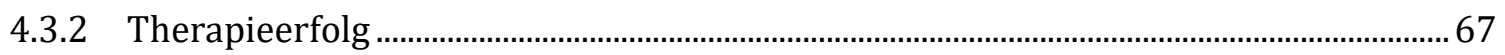

4.3.3 Untersuchung des Einflusses therapiebezogener Parameter auf die BMI-SDS-

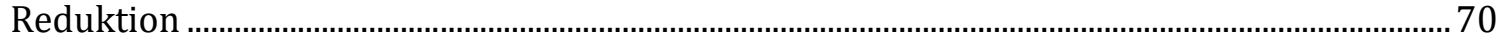

4.3.4 Untersuchung des Einflusses therapieunabhängiger Parameter auf die BMI-SDS-

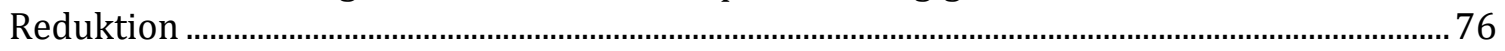

4.3.5 Untersuchung des Einflusses sonstiger Parameter auf die BMI-SDS-Reduktion........84

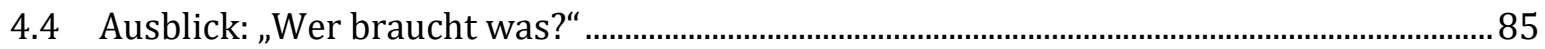

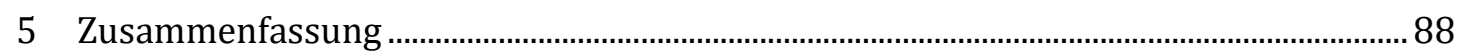

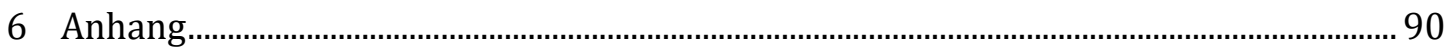

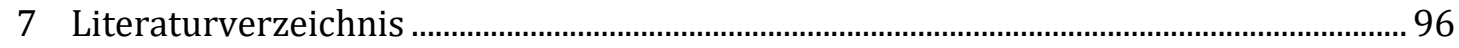

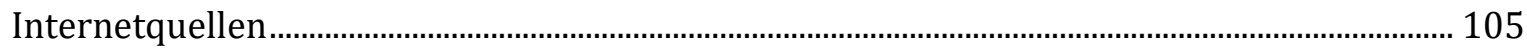




\section{Abbildungsverzeichnis}

Abbildung 1: Perzentilkurven für den Body-Mass-Index von Jungen und Mädchen im Alter von 0 - 18

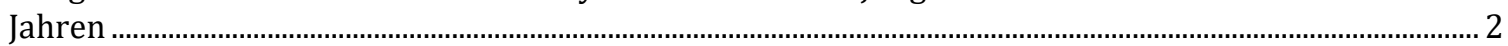

Abbildung 2: Vergleich der BMI-Perzentile für Jungen nach KiGG und Kromeyer-Hauschild ........................... 3

Abbildung 3: Vergleich der BMI-Perzentile für Mädchen nach KiGGS und Kromeyer-Hauschild ..................... 3

Abbildung 4: Flussdiagramm mit Darstellung des jeweiligen Therapieziels in Abhängigkeit vom jeweiligen Alter, BMI-Perzentil und Bestehen von Begleiterkrankungen .................................................................... 9

Abbildung 5: Kriterien zur Indikation übergewichtiger Kinder und Jugendlicher in eine Adipositasschulung (grau unterlegte Felder: Schulung indiziert) ....................................................................12

Abbildung 6: Patienteneinteilung entsprechend der BABELUGA-Methode in die Gruppen A-D anhand des erhobenen Risikoprofils mit Beschreibung des jeweiligen Therapievorgehens (aus: BABELUGA, o.J.)

Abbildung 7: Schematisches Konzept der eingesetzten Therapiemodalitäten entsprechend der Gruppeneinteilung im Rahmen der BABELUGA-Methode. Dabei ist neben den zum Einsatz kommenden Modalitäten auch die jeweilig angedachte Therapiedauer dargestellt (aus: BABELUGA, o.J.).

Abbildung 8: Flussdiagramm mit Darstellung der Patientenzahlen der in die Studie aufgenommenen Patienten, von der Teilnahme ausgeschlossenen Patienten sowie der Loss-to-Follow-up-Daten.........30

Abbildung 9: Gegenüberstellung der durchschnittlichen BMI-SDS-Reduktionen über Therapiezeit, postinterventionelle Zeit und Gesamtverlauf nach pP- und ITT-Analyse ......................................................30

Abbildung 10: Prozentuale Verteilung der Patienten über die verschiedenen Therapiekonzepte ..................32

Abbildung 11: Durchschnittliche Terminanzahl und Therapiedauer in Abhängigkeit vom Therapiekonzept 33

Abbildung 12: Durchschnittliche kurzfristige, langfristige und gesamten BMI-SDS-Reduktion in

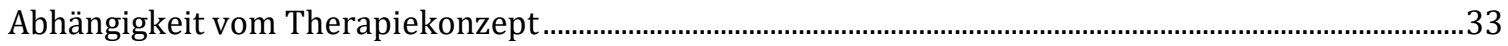

Abbildung 13: Lineare Regression mit Auftragung der BMI-SDS-Reduktion über den Therapieverlauf gegen die BMI-SDS-Reduktion über den gesamten Verlauf.........................................................................35

Abbildung 14: Lineare Regression mit Auftragung des BMI-SDS zum Zeitpunkt $\mathrm{T}_{2}$ gegen die BMI-SDSReduktion über den gesamten Therapiezeitraum ..

Abbildung 15: Kurzfristige BMI-SDS-Reduktion in Abhängigkeit von der Entwicklung von körperlicher Aktivität und Ausübung eines Vereinssports im Rahmen der Therapie

Abbildung 16: Lineare Regression mit Auftragung der Therapiedauer gegen die BMI-SDS- Reduktion über den gesamten Verlauf..

Abbildung 17: Durchschnittliche BMI-SDS-Reduktion über den Therapiezeitraum in Abhängigkeit vom Grund für die Beendigung der Therapie.

Abbildung 18: Lineare Regression mit Auftragung der kurzfristigen bzw. gesamten BMI-SDS-Reduktion gegen die Angaben zum Profit mit der ärztlichen Betreuung..

Abbildung 19: Lineare Regression mit Auftragung der kurzfristigen bzw. gesamten BMI-SDS-Reduktion gegen die Angaben zur Zufriedenheit mit der Ernährungsberatung

Abbildung 20: Lineare Regression mit Auftragung der kurzfristigen bzw. gesamten BMI-SDS-Reduktion gegen die Angaben zum Profit von der Ernährungsberatung....................................................................42

Abbildung 21: Vergleich der kurzfristigen, langfristigen und gesamten BMI-SDS-Reduktion zwischen Patienten mit dem Ziel einer Gewichtsreduktion bzw. -stabilisierung...

Abbildung 22: Durchschnittliche kurzfristige, langfristige und gesamte BMI-SDS-Reduktion der Patienten mit bzw. ohne konkrete Vorstellungen bezüglich des angestrebten Gewichtsverlusts vor Therapiebeginn..

Abbildung 23: Lineare Regression mit Auftragung der kurzfristigen BMI-SDS-Reduktion gegen das Alter bei Beginn der Therapie.

Abbildung 24: Lineare Regression mit Auftragung der langfristigen BMI-SDS-Reduktion gegen das Alter bei Beginn der Therapie.

Abbildung 25: Lineare Regression mit Auftragung der Rangkorrelation der BMI-SDS-Reduktion über den Gesamtverlauf gegen das Alter bei Therapiebeginn 
Abbildung 26: Lineare Regression mit Auftragung der kurzfristigen BMI-SDS-Reduktion gegen den BMISDS beim ersten Termin

Abbildung 27: Vergleichende Darstellung der kurzfristigen, langfristigen und gesamten BMI-SDSReduktion bei Jungen und Mädchen..

Abbildung 28: Vergleichende Darstellung der BMI-SDS-Reduktion über den Therapiezeitraum von Patienten mit bzw. ohne Vorliegen einer Insulinresistenz

Abbildung 29: Vergleichende Darstellung der durchschnittlichen kurzfristigen, langfristigen und gesamten BMI-SDS-Reduktion für Patienten mit bzw. ohne psychiatrische Diagnosen...

Abbildung 30: Durchschnittlich erreichte kurzfristige, langfristige und gesamte BMI-SDS-Reduktion von Patienten mit bzw. ohne psychiatrische Interventionen zusätzlich zur Adipositastherapie.

Abbildung 31: Vergleichende Darstellung der kurzfristigen und gesamten durchschnittlichen BMI-SDSReduktion der Patienten mit bzw. ohne Schulabschluss zum Zeitpunkt der Follow-up-Erhebung ......55

Abbildung 32: Vergleichende Darstellung der kurzfristigen und gesamten durchschnittlichen BMI-SDSReduktion von Patienten mit bzw. ohne Berufsausbildung zum Zeitpunkt der Follow-up-Erhebung 55

Abbildung 33: Vergleichende Darstellung der BMI-SDS-Reduktionen über die verschiedenen Zeiträume in Abhängigkeit von der mütterlichen Berufsausbildung.

Abbildung 34: Vergleichende Darstellung der BMI-SDS-Reduktionen über die verschiedenen Zeiträume in Abhängigkeit von der väterlichen Berufsausbildung

Abbildung 35: Lineare Regression mit Darstellung der kurzfristigen BMI-SDS-Reduktion in Abhängigkeit von der mütterlichen Wochenarbeitszeit

Abbildung 36: Lineare Regression mit Darstellung der langfristigen BMI-SDS-Reduktion in Abhängigkeit von der mütterlichen Wochenarbeitszeit

Abbildung 37: Lineare Regression mit Darstellung der BMI-SDS-Reduktion über den Gesamtverlauf in Abhängigkeit von der mütterlichen Wochenarbeitszeit .

Abbildung 38: Vergleichende Darstellung der kurzfristigen, langfristigen und gesamten BMI-SDSReduktion von in Einrichtungen lebenden Patienten im Vergleich zu Patienten in anderen Wohnsituationen.

Abbildung 39: Lineare Regression der Rangkorrelation mit Darstellung der kurzfristigen BMI-SDSReduktion in Abhängigkeit vom mütterlichen BMI.

Abbildung 40: Lineare Regression mit Auftragung der kurzfristigen BMI-SDS-Reduktion gegen den väterlichen BMI.

Abbildung 41: Durchschnittliche kurzfristige, langfristige und gesamte BMI-SDS-Reduktion von Patienten mit sportlicher Betätigung im Sportverein sowie von Patienten ohne sportliche Betätigung.

Abbildung 42: Vergleichende Darstellung des Prozentsatzes an Patienten mit Erreichen einer BMI-SDSReduktion von $\geq 0,2$ in der vorliegenden Studie sowie in der APV-Datenbank-Analyse nach Wiegand et al.

Abbildung 43: Kurzfristige, mittelfristige und langfristige BMI-SDS-Reduktion im Rahmen der EvAKuJStudie mit Darstellung des Prozentsatzes an Patienten mit BMI-SDS-Reduktion von $\geq 0,2, \geq 0,5,<0,2$ sowie der Drop-Out-Rate für ambulante und stationäre Patienten (Böhler et al. 2013)

Abbildung 44: Kurzfristiger, langfristiger und gesamter Prozentsatz an Patienten mit BMI-SDS-Reduktion $\leq 0 ;>0$, aber $<0,2 ; \geq 0,2$, aber $<0,5 ; \geq 0,5$

Abbildung 45: Behandlungskette für eine interdisziplinäre Adipositastherapie im Kindes- und Jugendalter entsprechend den Empfehlungen der KgAS (aus: Stachow et al. 2004) 


\section{Tabellenverzeichnis}

Tabelle 1: Untersuchte Parameter, die mithilfe von Patientenakten bzw. des krankenhausinternen elektronischen Patienteninformationssystem erhoben wurden

Tabelle 2: Untersuchte Parameter, die postalisch bzw. telefonisch mittels Fragebogen erfasst wurden .....26

Tabelle 3: Durchgeführte statistische Tests auf signifikante Zusammenhänge zwischen den verschiedenen Parametern und der BMI-SDS-Reduktion in Abhängigkeit von der Art des jeweiligen Datensatzes....27

Tabelle 4: Durchgeführte statistische Tests auf signifikante Zusammenhänge zwischen den verschiedenen Parametern (als unabhängige Variable) in Abhängigkeit von der Art des jeweiligen Datensatzes ......27

Tabelle 5: Durchschnittlich erreichte kurzfristige, langfristige und gesamte BMI-SDS-Reduktion der eingeschlossenen Patienten nach pP-Analyse ............................................................................................30

Tabelle 6: Durchschnittlich erreichte kurzfristige, langfristige und gesamte BMI-SDS-Reduktion der

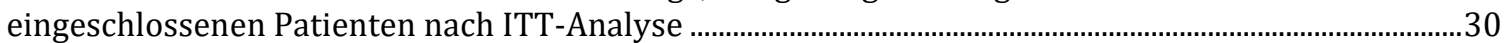

Tabelle 7: Kurzfristige, langfristige und gesamte Prozentsätze der Patienten ohne Gewichtsreduktion, mit Gewichtsreduktion, mit guter sowie sehr guter Gewichtsreduktion nach pP- und ITT-Analyse ...........31

Tabelle 8: Retrospektive Patientenangaben zur Entwicklung des Bewegungs- und Freizeitverhaltens.......31

Tabelle 9: Durchschnittliche Dauer, postinterventionelle Zeit und Terminzahl der durchgeführten Therapie unter Angabe des Konfidenzintervalls. .............................................................................................

Tabelle 10: Prozentsatz der jeweilig durchgeführten Therapie von allen Behandlungen der eingeschlossenen Patienten mit Angabe von Terminzahl und Therapiedauer sowie des entsprechenden Konfidenzintervalls

32

Tabelle 11: Darstellung der kurzfristigen, langfristig und gesamten durchschnittlichen BMI-SDSReduktion bzw. Erfolgsgruppen in Abhängigkeit vom jeweiligen Therapiekonzept.................................34

Tabelle 12: Signifikante Zusammenhänge zwischen Therapiekonzept und weiteren Parametern mit signifikanter Assoziation zur BMI-SDS-Reduktion mit Angabe des p-Werts.

Tabelle 13: Signifikante Zusammenhänge zwischen der Angabe zur Entwicklung der körperlichen Aktivität über die Therapie und weiteren ordinalen und metrischen Parametern mit signifikanter Assoziation zur BMI-SDS-Reduktion.

Tabelle 14: Signifikante Zusammenhänge zwischen der Angabe zur Entwicklung der körperlichen Aktivität durch die Therapie und weiteren nominalen Parametern mit signifikanter Assoziation zur BMI-SDS-Reduktion über mindestens einen der drei Zeiträume

Tabelle 15: Signifikante Zusammenhänge zwischen der Angabe zur Entwicklung der Aktivität im Sportverein durch die Therapie und weiteren ordinalen und metrischen Parametern mit signifikanter Assoziation zur BMI-SDS-Reduktion

Tabelle 16: Darstellung der Gründe für die jeweilige Therapie-Beendigung der zum Follow-up-Zeitpunkt befragten Patienten.

Tabelle 17: Durchschnittliche Bewertung der Abteilungen/ Therapeuten des SPZ zum Follow-upZeitpunkt mit Angabe von Zufriedenheit mit und Profit durch die einzelnen Instanzen.........................40

Tabelle 18: Positive und negative Kritik an ärztlicher Betreuung, Ernährungsberatung und psychologischer Betreuung der zum Follow-up-Zeitpunkt befragten Patienten........................................40

Tabelle 19: Signifikante Zusammenhänge zwischen den Angaben zum Profit von der ärztlichen Betreuung und weiteren ordinalen Parametern mit signifikanter Assoziation zur BMI-SDS-Reduktion.................42

Tabelle 20: Signifikante Zusammenhänge zwischen den Angaben zum Profit von der ärztlichen Betreuung und weiteren nominalen Parametern mit signifikanter Assoziation zur BMI-SDS-Reduktion................42

Tabelle 21: Signifikante Zusammenhänge zwischen den Angaben zur Zufriedenheit mit der Ernährungsberatung und weiteren Parametern mit signifikanter Assoziation zur BMI-SDS-Reduktion

Tabelle 22: Signifikante Zusammenhänge zwischen den Angaben zum Profit von der Ernährungsberatung und weiteren Parametern mit signifikanter Assoziation zur BMI-SDS-Reduktion ....................................43

Tabelle 23: Befragungsergebnisse bezüglich der Entscheidung zum Therapiebeginn ......................................44

Tabelle 24: Signifikanter Zusammenhang zwischen dem Vorhandensein eines konkreten Zielgewichts vor Therapiebeginn und dem nominalen Parameter „Therapiekonzept". 
Tabelle 25: Signifikanter Zusammenhang zwischen dem Vorhandensein eines konkreten Zielgewichts vor Therapiebeginn und dem ordinalen Parameter „Profit von ärztlicher Bewertung“ .46

Tabelle 26: Signifikante Zusammenhänge zwischen Alter bei Therapiebeginn und weiteren ordinalen und metrsichen Parametern mit signifikanter Assoziation zur BMI-SDS-Reduktion ..... 48

Tabelle 27: Signifikanter Zusammenhang zwischen Alter bei Therapiebeginn und dem nominalen Parameter „Mitgliedschaft im Sportverein“ . .48

Tabelle 28: Signifikante Zusammenhänge zwischen Patientengeschlecht und weiteren Parametern mit signifikanter Assoziation zur BMI-SDS-Reduktion

Tabelle 29: Darstellung der häufigsten Diagnosen der eingegangenen Patienten 51

Tabelle 30: Signifikante Zusammenhänge zwischen Vorliegen einer Insulinresistenz und weiteren metrischen und nominalen Parametern mit signifikanter Assoziation zur BMI-SDS-Reduktion...........52

Tabelle 31: Signifikante Zusammenhänge zwischen dem Erhalt zusätzlicher psychiatrischer Interventionen und weiteren Parametern mit signifikanter Assoziation zur BMI-SDS-Reduktion ......53

Tabelle 32: Vergleichende Darstellung der erreichten durchschnittlichen BMI-SDS-Reduktionen über die verschiedenen Zeiträume von Patienten mit bzw. ohne Ausübung einer sportlichen Aktivität um den Zeitpunkt der Therapie herum bzw. um den Follow-up-Zeitpunkt herum 61

Tabelle 33: Signifikante Zusammenhänge zwischen der Angabe einer Mitgliedschaft im Sportverein und weiteren Parametern mit signifikantem Einfluss auf die BMI-SDS-Reduktion. . .62 


\section{Abkürzungsverzeichnis}

AGA

APV

BABELUGA

Eigeniniti-

Gruppenthera

Abnehmen

BMGS

BMI

BMI-SDS

CE

DEXA

ECOG

EK

EvAKuJ „Evaluation der Adipositastherapie bei Kindern und Jugendlichen in Deutschland“" (Beobachtungsstudie)

FITOC

FKE

FM

IOTF

ITT

KFA

$\mathrm{KgAS}$

$\mathrm{KI}$

KiGGS

ME

NAFLD

NASH

NNT

PCO

$\mathrm{pP}$

RKI

SD

SES

Arbeitsgemeinschaft Adipositas im Kindes- und Jugendalter

Adipositas-Patienten-Verlaufsdokumentation

Berliner-Adipositas-Therapie-Programm für Kinder, Jugendliche und ihre Familien - Bewegung, Beratung, Begleitung - Essen und Trinken, ative - Lernen, Lebensqualität - Unterstützung der Familie pie für Kinder und Eltern - Adipositas-Diagnostik, langfristiges

Bundesministerium für Gesundheit und Soziale Sicherung

Body-Mass-Index

Body-Mass-Index Standard-Deviation-Score

Consistent Evidence

Dual-Röntgen-Absorptiometrie

European Childhood Obesity Group

Evidenzklasse

Freiburg Intervention Trial for Obese Children

Forschungsinstitut für Kinderernährung

Fettmasse

International Obesity Task Force

Intention to treat

Körperfettanteil

Konsensusgruppe Adipositasschulung im Kindes- und Jugendalter

Konfidenzintervall

Kinder- und Jugendgesundheitssurvey

Mixed evidence

Nicht-alkoholbedingte Fettlebererkrankung

Steatohepatitis

Number needed to treat

Polyzystisches Ovar-Syndrom

Per Protocol

Robert Koch-Institut

Standardabweichung

Socioeconomic Status 
SPZ

WHO
Sozialpädiatrisches Zentrum

World Health Organization 


\section{Einleitung}

Die vorliegende Arbeit beschäftigt sich mit Langzeitverläufen von Adipositastherapie im Kindesund Jugendalter. Dabei steht die Frage nach Prädiktoren im Vordergrund, die einen positiven oder negativen Einfluss auf den Therapieerfolg haben. Während eine Vielzahl an Studien sich mit dem Therapieerfolg nach kurzzeitigen Interventionen beschäftigt, ist die Zahl an Arbeiten mit dem Fokus auf Langzeitverläufen vergleichsweise gering. Das Wissen um Prädiktoren für den langfristigen Therapieerfolg ist jedoch insofern von Bedeutung, als dadurch mithilfe von modifizierten und individualisierten Therapieansätzen eine Optimierung der Therapie ermöglicht werden kann.

\subsection{Definition von Adipositas im Kindes- und Jugendalter}

\subsubsection{Definition}

Adipositas ist definiert als pathologische Erhöhung des Körperfettanteils (KFA) im Verhältnis zur Gesamtkörpermasse (Wabitsch und Kunze 2015). Im Hinblick auf diese Definition stellt sich die Frage, auf welche Art und Weise der KFA bestimmt werden kann. Es existieren verschiedene Mess- beziehungsweise Annäherungsmethoden, die im Folgenden besprochen werden.

\subsubsection{Methoden zur Bestimmung des Körperfettanteils}

Einige sehr genaue Methoden zur Bestimmung des KFA sind mit einem relativ hohen Aufwand verbunden, wodurch sie im klinischen Alltag wenig Anwendung finden. $\mathrm{Zu}$ diesen zählt beispielsweise die Hyperdensitometrie (Goulding et al. 1996; Pietrobelli und Tato 2005), bei der der Patient ${ }^{1}$ in einem Wassertank gewogen wird, um so auf die Körperdichte zu schließen. Dasselbe gilt für die Dual-Röntgen-Absorptiometrie (DEXA), bei der die Bestimmung des KFA mithilfe niedrigenergetischer Röntgenstrahlung erfolgt (Chan 1992). Weitere ScanningMethoden sind CT und MRT, wobei insbesondere die CT aufgrund hoher Strahlendosen für Kinder eher ungeeignet ist (Pietrobelli und Tato 2005). Praktikabler und kostengünstiger, wenn auch etwas weniger genau, ist die Bioelektrische Impedanzanalyse: Über mehrere Elektroden wird ein Wechselstrom durch den Körper geleitet, mit dessen Hilfe der weniger leitfähige Fettanteil des Körpers erfasst wird (McCarthy et al. 2006). Ebenfalls kostengünstig und im klinischen Alltag einsetzbar ist die anthropometrische Methode der Hautfaltendickenmessung: Mithilfe einer Körperfettzange (Caliper) werden in bestimmten Messlokalisationen (z. B. an Bizeps, Trizeps sowie subskapulär) Hautfalten gebildet und gemessen, von denen auf den KFA rückgeschlossen wird (Kromeyer-Hauschild et al. 2001; Pietrobelli und Tato 2005). Bei hohen Messfehlerraten weist diese Technik jedoch eine niedrige Reproduzierbarkeit auf (KromeyerHauschild et al. 2001).

Zusätzlich zu den direkten Messmethoden existieren Indizes zur Abschätzung des KFA. Am verbreitetsten ist in diesem Kontext der Body-Mass-Index (BMI). Er errechnet sich mithilfe der Formel Körpergewicht $(\mathrm{kg}) /$ Körperhöhe $(\mathrm{m})^{2}$. Es ist vielfach belegt, dass dieser sich als Maß zur Annäherung des KFA eignet. Ein Zusammenhang mit der Hautfaltendicke wurde ebenso nachgewiesen (Spyckerelle et al. 1988) wie eine Korrelation zwischen BMI und mittels DEXA ermitteltem prozentualem KFA (Daniels et al. 1997; Pietrobelli et al. 1998). Weiterhin existieren Studien, die eine enge positive Korrelation zwischen BMI und dem Auftreten von metabolischen Risikofaktoren nahelegen (Guillaume 1999; Zwiauer et al. 1992).

Dennoch weist auch der BMI Limitationen auf. Grund hierfür ist, dass keine direkte Messung des Fettanteils erfolgt, sodass ggf. ergänzende Untersuchungen erfolgen sollten (Rolland-Cachera

${ }^{1}$ Zu Gunsten einer besseren Lesbarkeit wird in der vorliegenden Arbeit durchgehend die männliche Form „Patient" verwendet, gemeint sind hiermit jedoch beide Geschlechter. 
2011). Ein höherer BMI bei gleicher Größe kann beispielsweise auch aus einem höheren Anteil an Muskelmasse oder Unterschieden im Körperbau resultieren (Daniels et al. 1997; KromeyerHauschild et al. 2001).

Insgesamt stellt der BMI jedoch eine simple Möglichkeit dar, die Gesamtkörperfettmasse mit nur minimalem Zeit- und Kostenaufwand einzuschätzen und ist momentan als Index zur Evaluation von Übergewicht und Adipositas allgemein anerkannt. Seine Anwendung wird von der International Obesity Task Force (IOTF), European Childhood Obesity Group (ECOG) und auf nationaler Ebene von der Arbeitsgemeinschaft Adipositas im Kindes- und Jugendalter (AGA) zur Definition von Adipositas empfohlen und ist auch der vorliegenden Arbeit zugrunde gelegt (Lobstein et al. 2005; Poskitt 1995; Wabitsch und Kunze 2015).

\subsubsection{BMI-Perzentile}

Dennoch kann der BMI nicht als alleinstehender Maßstab zur Definition von Adipositas im Kindes- und Jugendalter herangezogen werden. Grund ist, dass er in Bezug auf Geschlecht, sexuellen Reifegrad und Alter variiert (Daniels et al. 1997; Mast et al. 1998). Dementsprechend kommt es mit zunehmendem Alter zu physiologischen Schwankungen des BMI, die sich aus Veränderungen des Verhältnisses von Fettmasse zu fettfreier Masse im Rahmen von Wachstum und sexueller Entwicklung ergeben (Daniels et al. 1997; Goulding et al. 1996).

Um dieser Problematik entgegenzuwirken, vergleicht man BMI-Werte von Kindern und Jugendlichen mit denen einer Referenzpopulation. Für diese Referenzpopulationen werden Referenzkurven (Perzentile) erstellt, die den Verlauf des BMI über das Alter jeweils für beide Geschlechter darstellen. So kann eine alters- und geschlechtsspezifische Einordnung individueller BMI-Werte erfolgen. Als Beispiel sind auf der folgenden Abbildung von KromeyerHauschild et al. erstellte BMI-Perzentile dargestellt, die auch als Referenz für die vorliegende Arbeit genutzt wurden (aus: Kromeyer-Hauschild et al. 2001):
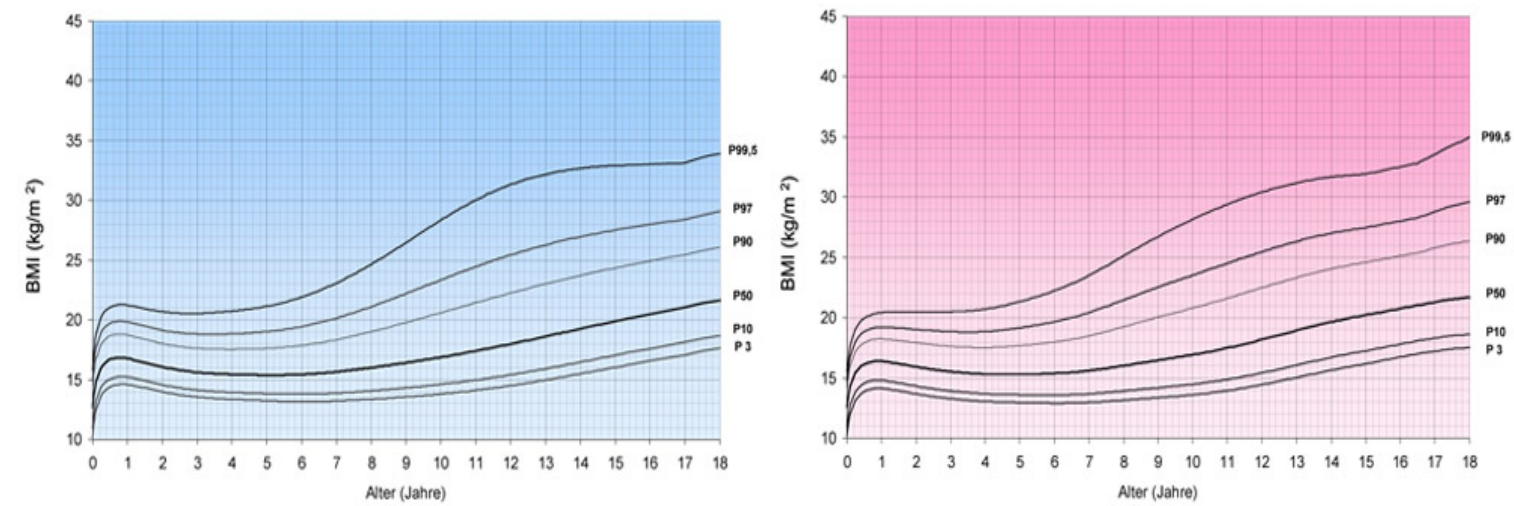

Abbildung 1: Perzentilkurven für den Body-Mass-Index von Jungen (links) und Mädchen (rechts) im Alter von 0 - 18 Jahren

Es zeigen sich ähnliche altersabhängige Veränderungen bei Jungen und bei Mädchen. Bei beiden Geschlechtern steigt der BMI nach der Geburt an und erreicht sein Maximum bei Jungen etwa im Alter von acht, bei Mädchen im Alter von neun Monaten. Daraufhin kommt es zu einem erneuten Abfall. Im Alter von fünf Jahren und einem Monat bei Jungen und vier Jahren und fünf Monaten bei Mädchen erreicht der BMI sein Minimum. Im Anschluss kommt es zu einem erneuten Anstieg, der als adiposity-rebound bezeichnet wird (Kromeyer-Hauschild et al. 2001). Ein früher adiposity- rebound ist dabei mit einem erhöhten Risiko für Adipositas im Erwachsenenalter assoziiert (Whitaker et al. 1998). Beim BMI der Mädchen kommt es etwa ab dem Alter von 15 Jahren zu einer Abflachung, der der Jungen steigt weiterhin an (Kromeyer-Hauschild et al. 2001).

Es existieren verschiedene, sowohl nationale als auch internationale Referenzkurven. Auf internationaler Ebene sind insbesondere die Perzentilkurven der IOTF und der World Health 
Organization (WHO) zu nennen: Die Kurven der IOTF beruhen auf einer Arbeit von Cole et al. aus dem Jahr 2000 und umfassen Daten aus sechs verschiedenen Ländern (Cole et al. 2000). Die aktuellen Referenzwerte der WHO basieren auf verschiedenen Referenzpopulationen: Für unter Fünfjährige wurden Daten gesunder Kinder aus ebenfalls sechs verschiedenen Ländern erfasst, die Kurven der höheren Altersklassen beruhen auf Daten US-amerikanischer Kinder und Jugendlicher (de Onis et al. 2007; WHO 2006). Internationale Daten ermöglichen die Setzung allgemeingültiger Standards und eine überregionale Vergleichbarkeit, können dabei jedoch ethnische und nationale Unterschiede übergehen.

Daneben existieren für verschiedene Länder diverse nationale Referenzen (Cole et al. 1995; Lindgren et al. 1995; Rolland-Cachera et al. 1991). In Deutschland empfiehlt die AGA in ihren Leitlinien die Anwendung der schon erwähnten Referenzwerte von Kromeyer-Hauschild et al. (Kromeyer-Hauschild et al. 2001; Wabitsch und Kunze 2015). Diese basieren auf den Ergebnissen von 17 Studien, die von 1985 bis 1999 erhoben wurden, und Daten von 17.147 Jungen und 17.275 Mädchen aus unterschiedlichen Regionen Deutschlands einschließen (Kromeyer-Hauschild et al. 2015). Im Jahr 2015 erfolgte eine Anpassung der Perzentilwerte der 15- bis 18-jährigen, sodass diese nun eine kontinuierliche Betrachtung bis ins Erwachsenenalter ermöglichen (Kromeyer-Hauschild et al. 2015).

Die Perzentile nach Kromeyer-Hauschild et al. sind jedoch aufgrund der Heterogenität der eingegangenen Datensätze nicht gänzlich repräsentativ (RKI 2013; Rosario et al. 2010). Beispielsweise beruhen die Gewichts- und Größenangaben teils auf Messungen und teils auf Selbstangaben und stammen zudem aus unterschiedlichen Zeiträumen (Rosario et al. 2010). Um diesem Mangel an repräsentativen Datensätzen zu begegnen, führte das Robert Koch-Institut (RKI) von 2003 bis 2006 die Basiserhebung der „Studie zur Gesundheit von Kindern und Jugendlichen in Deutschland“ (damals noch "Kinder- und Jugendgesundheitssurvey“ (KiGGS)) mit Einschluss von 17.641 Jungen und Mädchen aus 167 Orten Deutschlands durch (Kurth 2007). Durch Einsatz standardisierter und qualitätskontrollierter Messverfahren entstand ein repräsentativer Datensatz - die erhobenen Perzentile sind folglich denen nach KromeyerHauschild et al. prinzipiell vorzuziehen.

Vergleicht man die Kromeyer-Hauschild-Perzentile mit denen der KiGGS-Studie, zeigen die Kurven zwar einen recht ähnlichen Verlauf, die KiGGS-Perzentile weisen allerdings ab dem sechsten Lebensjahr eine Verschiebung nach oben auf (Abbildungen 2 und 3) (aus: Kurth und Schaffrath Rosario 2007):

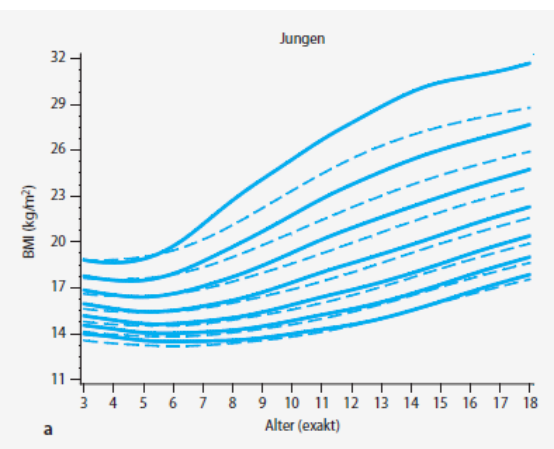

Abbildung 2: Vergleich der BMI-Perzentile für Jungen nach KiGGS (durchgezogene Linie) und KromeyerHauschild (gestrichelte Linie)

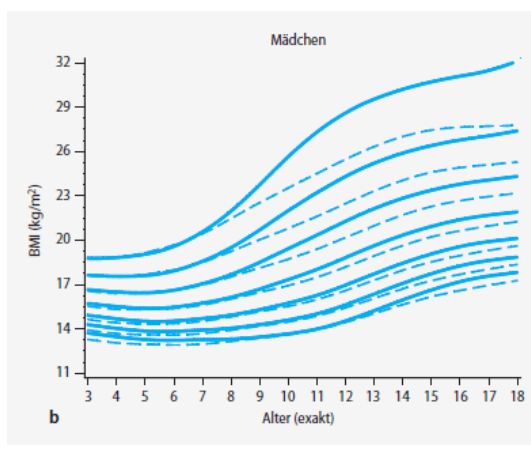

Abbildung 3: Vergleich der BMI-Perzentile für Mädchen nach KiGGS (durchgezogene Linie) und KromeyerHauschild (gestrichelte Linie)

Im Vergleich zu den Daten von Kromeyer-Hauschild et al. sind im KiGGS-Datensatz 50 \% mehr Kinder übergewichtig (RKI 2013). Würde man die Perzentile der KiGGS-Studie nun als neue Referenz verwenden, würde dies also einen offensichtlich stattgehabten Aufwärtstrend der Adipositasprävalenz verschleiern. Aus diesem Grund empfehlen sowohl die Leitlinien der AGA als auch das Robert Koch-Institut weiterhin die Verwendung der Perzentile nach Kromeyer- 
Hauschild et al. als Referenz (RKI 2013; Wabitsch und Kunze 2015). Diese sind auch der vorliegenden Arbeit zugrunde gelegt.

\subsubsection{BMI-SDS}

Die Einordnung des BMI anhand von Perzentilen ist ein geeignetes Maß, um interindividuelle Vergleiche im Bezug auf eine Referenzpopulation anzustellen (Pietrobelli et al. 1998). Allerdings eignet sich der BMI nur bedingt, um individuelle Gewichtsentwicklungen aufzuzeigen. Der Grund hierfür ist, dass nicht nur eine relative Gewichtsreduktion sondern auch eine Stagnation oder sogar leichte Gewichtszunahme in erniedrigten BMI-Perzentilen resultieren können, da die Perzentilkurven mit dem Alter ansteigen (Barlow 2007). Außerdem sind Veränderungen, die sich oberhalb der 99.9 Perzentile abspielen, nicht ersichtlich (Kromeyer-Hauschild et al. 2001). Aus diesem Grund empfiehlt sich die Anwendung von BMI-Standard-Deviation-Scores (BMISDS). Diese geben die Standardabweichung des untersuchten BMI vom alters- und geschlechtsspezifischen Median der Referenzpopulation an. So können individuelle Gewichtsverläufe sensibler dargestellt werden (Kromeyer-Hauschild et al. 2001). Eine genauere Erläuterung der Anwendung von BMI-SDS folgt im Kapitel „Material und Methoden“.

\subsubsection{Cut-Offs und Terminologie}

Im Hinblick auf die oben genannte Definition von Adipositas stellt sich nun die Frage, wie hoch der KFA (bzw. als indirektes Maß: der BMI) sein muss, um als pathologisch zu gelten. Die Festlegung eines genauen Cut-Offs gestaltet sich jedoch bei Kindern und Jugendlichen problematisch. So bestehen bei Erwachsenen klare Richtwerte, die Adipositas definieren. Übergewicht besteht ab einem BMI von mindestens 25, Adipositas ab einem BMI von mindestens 30. Eine Überschreitung dieser Grenzwerte ist mit einer Erhöhung des Risikos für Komorbiditäten und Folgeerkrankungen assoziiert (WHO 2000). Bei Kindern und Jugendlichen kommt es jedoch seltener und später zu Folgeerkrankungen durch Adipositas. Es ist folglich schwieriger, einen Grenzwert festzusetzen, ab dem von einem erhöhten Gesundheitsrisiko ausgegangen werden kann (Cole et al. 2000; Wabitsch und Kunze 2015). Aus diesem Grund werden von verschiedenen Instanzen unterschiedliche Grenzwerte zur Definition kindlicher Adipositas empfohlen. Diese werden oft in Form von BMI-Perzentilen oder Standardabweichungen (SD) angegeben und beziehen sich auf die jeweilige Referenzpopulation. Die IOTF empfiehlt beispielsweise anhand der Perzentilen von Cole et al. diejenigen Werte als Grenzwerte zu verwenden, die einen kontinuierlichen Übergang in die Grenzwerte Erwachsener beschreiben (Cole et al. 2000). Die WHO definiert Übergewicht für unter Fünfjährige als BMI von $>+2$ SD. Bei Kindern ab fünf Jahren dagegen besteht schon bei $>+1$ SD Übergewicht, bei einem BMI > +2 SD Adipositas (de Onis et al. 2007; WHO 2006, 2007).

In Deutschland liegt nach den Leitlinien der AGA Übergewicht ab einem BMI über dem 90. Perzentil vor. Ein BMI über dem 97. Perzentil ist definiert als Adipositas, über dem 99.5. Perzentil als extreme Adipositas. Da die Grenzwerte anhand der statistischen Verteilung des BMI gesetzt wurden, besteht auch hier ein beinahe gleichmäßiger Übergang zu den Werten für Erwachsene. Dabei entspricht das 90. Perzentil in etwa +1 SD, das 97. Perzentil circa +2 SD (Wabitsch und Kunze 2015). Diese Werte wurden auch für die vorliegende Arbeit genutzt.

\subsection{Prävalenz in Deutschland}

Mit der KiGGS-Studie, die Daten aus den Jahren 2003 bis 2006 beinhaltet, wurden erstmals bundesweit repräsentative Prävalenzzahlen erhoben (Kurth und Schaffrath Rosario 2007). Nach den Definitionen der AGA waren zum Zeitpunkt der Erhebung $15 \%$ der Kinder und Jugendlichen übergewichtig, davon 6,3 \% adipös. In absoluten Zahlen waren also 1,1 Millionen Kinder in Deutschland übergewichtig und weitere 800.000 adipös. Dabei ist die Prävalenz altersabhängig: Mit zunehmendem Alter steigt der Anteil übergewichtiger und adipöser Kinder und Jugendlicher an (Übergewicht: von $9 \%$ bei den 3- bis 6-jährigen auf bis zu $17 \%$ bei den 14- 
bis 17-jährigen; Adipositas: von 2,9 \% bei den 3- bis 6-jährigen auf 8,5 \% bei den 14- bis 17 jährigen) (Kurth und Schaffrath Rosario 2007).

Vergleicht man diese Daten mit solchen aus den 1980er Jahren, zeigt sich ein Anstieg der Prävalenz von Übergewicht um 50 \%, von Adipositas sogar um 100 \% (Wabitsch et al. 2013). Da auch international ähnliche Verläufe zu verzeichnen sind, spricht die WHO von einer globalen Adipositas-„Epidemie“ (WHO 2000). In Deutschland wird dieser Trend, wie bereits im Abschnitt „Perzentile“ besprochen, auch im Vergleich der Perzentile von Kromeyer-Hauschild et al. mit denen der KiGGS-Studie deutlich (Abbildung 2 und 3). Beim Vergleich der Kurven fällt auf, dass die Prävalenz bis zum Alter von sechs Jahren weitestgehend unverändert ist. Mit zunehmendem Alter findet jedoch eine stärkere Verschiebung der Kurven nach oben statt. Der Prävalenzanstieg ist außerdem perzentilabhängig: Während die unteren Perzentilkurven fast unverändert sind, findet mit steigender Perzentilklasse auch eine zunehmende Verschiebung nach oben statt. Dies bedeutet, dass vom Aufwärtstrend gerade die ohnehin schon übergewichtigen bzw. adipösen Perzentilbereiche betroffen sind (Kurth und Schaffrath Rosario 2007).

Die höchsten Prävalenzen der KiGGS-Studie für Übergewicht und Adipositas sind für Familien mit niedrigem sozioökonomischen Status (englisch: „,socioeconomic status“, SES) sowie Kindern mit Migrationshintergrund zu verzeichnen (Kurth und Schaffrath Rosario 2007). Dennoch erstreckt sich - bei unterschiedlichen Ausgangswerten - der Prävalenzanstieg über alle Bildungsniveaus und betrifft alle sozialen Schichten, wie Toschke et al. nachweisen konnten (Toschke et al. 2005).

Da seit der KiGGS-Studie keine entsprechenden Erhebungen mehr vorgenommen worden sind, liegen für aktuellere Prävalenzen keine vergleichbaren Daten vor. Allerdings untersuchten Moss et al. die Entwicklung der Daten aus Schuleingangsuntersuchungen bis zum Jahr 2009 (Moss et al. 2012; Moss et al. 2007). Da diese obligatorisch sind, liegen repräsentative Datensätze für die entsprechende Altersklasse vor. Während es für zehn Jahre bis zum Jahr 2004 zu einem stetigen Anstieg der Prävalenzen von Übergewicht und Adipositas gekommen war, stagnierten die Werte zwischen 2004 und 2008 (Moss et al. 2012; Moss et al. 2007). In manchen Bundesländern war sogar ein Abfall der Prävalenzen zu verzeichnen (Moss et al. 2012).

Eine ähnliche Entwicklung konnte auch für andere - sowohl europäische als auch nichteuropäische - Länder festgestellt werden (Lioret et al. 2009; Matthiessen et al. 2008; Ogden et al. 2008; Olds et al. 2010; Sundblom et al. 2008; Tambalis et al. 2010). Für diese Entwicklung existieren verschiedene Erklärungsansätze: Während Tambalis et al. von einem Ceiling-Effekt ausgehen (Tambalis et al. 2010) halten Moss et al. verschiedene seit den 1990er Jahren ins Leben gerufene Präventionsmaßnahmen für ursächlich.

In den Jahren 2009 bis 2012 erfolgte eine Follow-up-Erhebung der KiGGS-Studie für Kinder und Jugendliche im Alter von 11 bis 17 Jahren („KiGGS Welle 1“) (Brettschneider et al. 2015). Diese ist zwar in ihrer Methodik nicht gänzlich vergleichbar, da der Großteil der Datenerhebung im Selbstbericht erfolgte, gibt aber dennoch einen Überblick über die aktuelle Prävalenz in diesen Altersklassen. Auch hier lässt sich eine Stagnation der Prävalenz von Übergewicht im Vergleich zu den früheren KiGGS-Daten beobachten. Die Adipositasprävalenz zeigt im Gegensatz dazu allerdings bei beiden Geschlechtern einen leichten Anstieg (von 8,9\% auf $10 \%$ ). Nach Wabitsch et al. ist in diesem Zusammenhang für die nächsten Jahre trotz Stagnationsphänomenen keine Rückläufigkeit der Prävalenzen von Übergewicht und Adipositas im Kindes- und Jugendalter zu erwarten (Wabitsch et al. 2013).

\subsection{Ursachen und Folgen von Adipositas}

\subsubsection{Ursachen}

Es existiert eine Vielzahl an Ursachen, die zur Entstehung dieser hohen Prävalenzzahlen beigetragen haben. Grundsätzlich liegt der Ursprung der Adipositas im Energiestoffwechsel: Übersteigt die Energieaufnahme den Energieverbrauch, so kommt es zur Gewichtszunahme. Dies kann verschiedene Gründe haben: 
Nur in etwa ein bis zwei Prozent der Fälle kann, im Sinne einer sekundären Adipositas, eine zugrunde liegende Erkrankung ausgemacht werden (Reinehr et al. 2007; Roth et al. 2002). Hierzu gehören beispielsweise endokrine Störungen wie das Cushing-Syndrom oder das hypothalamische Syndrom. Auch genetische Syndrome und Mutationen wie das Prader-Willioder Bardet-Biedl-Syndrom sowie Mutationen im Leptin-Melanocortin-System können verantwortlich sein (Anderson und Butcher 2006; Skelton et al. 2011; Wabitsch und Kunze 2015). Nicht zuletzt können verschiedene Medikamente Adipositas hervorrufen, hierzu zählen Antipsychotika, Glukokortikoide, Antikonvulsiva und Insuline (Correll et al. 2011; Skelton et al. 2011). Auch wenn diese sekundären Ursachen selten sind, sollten sie doch immer im Rahmen einer Adipostasdiagnostik ausgeschlossen werden (Wabitsch und Kunze 2015).

Die Ätiologie der wesentlich häufigeren primären Adipositas ist multifaktoriell. Oft wird zur Erläuterung der Entstehung von Übergewicht das „Nature and Nurture“ - (AAnlage und Umwelt") Modell herangezogen (Kitsantas et al. 2016; Kmietowicz 2015).

Zwillingsstudien und verschiedene andere Untersuchungen haben ergeben, dass der BMI zu einem großen Teil genetisch bedingt ist (MacDonald und Stunkard 1990; Wabitsch et al. 2013; WHO 2000). Nach der Hypothese des „thrifty genotype“ („sparsamer Genotyp“) haben sich im Laufe der Evolution Genvarianten durchgesetzt, die ein Überleben in Zeiten von Nahrungsknappheit ermöglichen. Unter den heutigen Bedingungen in den Industrienationen resultieren diese Genotypen jedoch eher in Übergewicht (Neel 1962).

Einige Faktoren, die zu einem individuell niedrigeren Energieumsatz führen können, sind bekannt. Dazu gehören z. B. Unterschiede in der „nonexercise-activity-thermogenesis“, die eine Form von Energieverbrauch in Abwesenheit von direkter körperlicher Aktivität durch eine Vielzahl kleiner Bewegungen im Alltag darstellt. Personen mit geringerer „nonexercise-activitythermogenesis" verbrauchen also verhältnismäßig weniger Energie (Levine et al. 1999). Außerdem konnte man interindividuelle Unterschiede im Energieverbrauch durch die Mitochondrien von Muskelzellen nachweisen (Lowell und Spiegelman 2000).

Dennoch bieten diese anlagebedingten Variationen keine ausreichende Erklärung für die rasante Zunahme der Prävalenz von Adipositas in den letzten Jahrzehnten.

Dementsprechend muss der Umwelt („Nurture“) die größere Rolle zufallen (Roth et al. 2002). Man kann sagen, dass sich die Entwicklung kindlicher Adipositas auf drei große Säulen stützt: das kindliche Essverhalten, die körperliche Aktivität und die Schlafgewohnheiten. Ein Essverhalten mit übermäßigem Konsum zucker- und fetthaltiger Lebensmittel wie hochkalorischer Snacks, zuckerhaltiger Getränke und Fast Food sowie Bewegungs- und Schlafmangel können die Entstehung von Adipositas begünstigen (Spruijt-Metz 2011; Wabitsch et al. 2013).

Auf diese Verhaltensweisen haben insbesondere die Eltern in ihrer Funktion als Autoritätspersonen und Vorbild einen großen Einfluss. So konnte bereits in mehreren Studien ein Zusammenhang zwischen elterlichem - insbesondere mütterlichem - und kindlichem BMI festgestellt werden. Dabei haben Kinder adipöser Eltern selbst ein höheres Risiko, adipös zu werden (Knerr et al. 2008; Whitaker et al. 1997). Ursächlich ist ein Zusammenspiel verschiedener Faktoren. Kramer et al. wiesen einen inversen Zusammenhang zwischen Stilldauer und Adipositas-Risiko nach (Kramer 1981). Zusätzlich scheint es zu einer Übertragung elterlicher Ernährungsgewohnheiten auf ihre Kinder zu kommen. Beispielsweise konnte ein Zusammenhang zwischen elterlichem und kindlichem Konsum von Fett, Obst und Gemüse nachgewiesen werden (van der Horst et al. 2007). Zudem werden Mahlzeiten zu einem zunehmend unregelmäßigem Rhythmus immer seltener im Kreis der Familie eingenommen, was mit verhältnismäßig höheren kindlichem BMI-Werten assoziiert ist (Roth et al. 2002; Story et al. 2008).

Auch der familiäre SES scheint sich invers auf den BMI auszuwirken (Röbl et al. 2013). Ein möglicher Grund ist, dass Kindern aus Familien mit niedrigerem SES einige gesundheitsbewusstere Verhaltensweisen verwehrt bleiben, wie etwa der Zugriff auf gesündere, jedoch teurere Lebensmittel (Kersting und Clausen 2007; Story et al. 2008). Außerdem konnte eine positive Korrelation zwischen elterlichem Bildungsstand (als Komponente des SES) und kindlichem Level an körperlicher Aktivität ausgemacht werden (Gordon-Larsen et al. 2000). 
Aber auch ohne Berücksichtigung dieser Faktoren scheint ein inverser Zusammenhang zwischen SES in sich und kindlichem BMI zu bestehen (Ogden et al. 2010).

Auch die dritte Säule, das Schlafverhalten, ist zu einem gewissen Grad abhängig vom elterlichen Verhalten. Dies ist insofern von Bedeutung, als kürzere Schlafzeiten das Risiko für kindliche Adipositas signifikant erhöhen (Fatima et al. 2015; Sekine et al. 2002). Neben der Bestimmung von Schlafenszeiten haben die Eltern auch Einfluss auf das Vorhandensein von Fernsehern und ähnlicher Technologie im Kinderzimmer. Dieses kann sich negativ auf das Schlafverhalten auswirken (Li et al. 2007).

Neben dem Schlafverhalten beeinflusst der Fernsehkonsum auch das Ernährungs- und Bewegungsverhalten. So korreliert die tägliche Durchschnitts-Fernsehdauer mit adipogenem Essverhalten und übermäßiger Nahrungsaufnahme (Coon et al. 2001). Außerdem konnte eine Assoziation zwischen einem hohen $\mathrm{Ma}$ an sitzenden Tätigkeiten wie Fernsehen und Computerspielen und geringerer körperlicher Aktivität sowie einem verhältnismäßig höheren BMI nachgewiesen werden (Andersen et al. 1998; Goran und Treuth 2001).

Diese Tendenzen werden von der Nahrungsmittelindustrie begünstigt, die ein hohes Angebot an preiswerten, energiereichen und ballaststoffarmen Produkten zur Verfügung stellt und durch entsprechendes Marketing (wie TV-Werbespots) vertreibt (Anderson und Butcher 2006; Caroli et al. 2004; Roth et al. 2002)

Daneben wirkt sich auch die so genannte built environment (etwa „Vom Menschen geschaffene Umwelt") auf den kindlichen BMI aus (Spruijt-Metz 2011). Dazu zählen beispielsweise auch Schulen. Da in oder im Umkreis der Schule häufig zumindest eine Mahlzeit am Tag eingenommen wird, besteht ein wesentlicher Einfluss auf die Ernährung von Kindern und Jugendlichen (Alviola et al. 2014).

\subsubsection{Folgen}

Die genannten Ursachen haben zu den hohen Prävalenzen von kindlicher Adipositas in den Industrienationen beigetragen. Dies ist insofern von Bedeutung, als kindliche Adipositas mit diversen gesundheitlichen Risiken assoziiert ist. Dabei kann man zwischen psychosozialen und körperlichen Folgen unterscheiden.

Die psychosozialen Beeinträchtigungen basieren insbesondere auf der sozialen Diskriminierung und Stigmatisierung, denen übergewichtige und adipöse Kinder oft ausgesetzt sind. Ursächlich ist die in unserer Gesellschaft vorherrschende Assoziation eines adipösen Körperbaus mit mangelnder Fitness und Gesundheit sowie charakterlichen Schwächen (Staffieri 1967). Die Folge sind ein geringes Selbstwertgefühl - insbesondere ab Eintritt in die Pubertät - und soziale Isolation (French et al. 1995; Wabitsch 2000). Die Folgen der kindlichen Adipositas können bis ins Erwachsenenalter reichen. Hier besteht eine Assoziation zu einem verhältnismäßig niedrigem SES bei geringerem durchschnittlichen Einkommen sowie niedrigeren Heiratsquoten (Gortmaker et al. 1993).

Neben den psychosozialen ist eine Vielzahl an körperlichen Komorbiditäten bekannt. Besonders häufig sind kardiovaskuläre Auswirkungen, das Risiko hierfür ist bei Bestehen eines abdominellen Fettverteilungsmusters noch verstärkt. Eine häufige Komplikation ist die arterielle Hypertonie. Flechtner-Mors et al. konnten in einer Studie mit Daten von 63.025 Kindern und Jugendlichen bei bis zu 45 \% der übergewichtigen und adipösen Kindern erhöhte Blutdruckwerte feststellen. Bei den Normalgewichtigen lag die Rate dagegen bei $6 \%$ (FlechtnerMors et al. 2012).

Weiterhin besteht ein Zusammenhang zwischen kindlicher Adipositas und Insulinresistenz. Diese kann wiederum zu Hyperinsulinämie und gestörter Glukosetoleranz führen (Zwiauer et al. 1989). Von letzterer sind ab Pubertätsbeginn etwa ein Drittel der übergewichtigen Kinder und Jugendlichen betroffen (AGA 2011). In diesem Zusammenhang konnte auch eine Zunahme der Inzidenz von kindlichem Typ 2-Diabetes festgestellt werden (maximal $1 \%$ der übergewichtigen Kinder und Jugendlichen) (AGA 2011; Pinhas-Hamiel et al. 1996). 
Ebenfalls häufig ist eine Dyslipidämie, betroffen sind etwa ein Viertel aller übergewichtigen Kinder und Jugendlichen (AGA 2011). Dabei kommt es zu erhöhten LDL- und Triglycerid- bei gleichzeitig verminderten HDL-Spiegeln (Reinehr et al. 2005; Vanhala et al. 1998). Bei Erwachsenen ist die Dyslipidämie ein bekannter Risikofaktor für die Entstehung von Atherosklerose (Whitman et al. 1997). Außerdem konnten Reinehr et al. eine Assoziation zwischen kindlicher Adipositas und einer Erhöhung der Intima-Media-Dicke feststellen. Diese gilt als Frühform der Atherosklerose und ist entsprechend mit einem erhöhten kardiovaskulären Risiko assoziiert (Reinehr und Kiess et al. 2006).

Etwa ein Fünftel der übergewichtigen Kinder und Jugendlichen weisen außerdem eine Hyperurikämie auf (AGA 2011; Civantos Modino et al. 2012).

Eine gastrointestinale Folgeerscheinung der kindlichen Adipositas ist die Nicht-alkoholbedingte Fettlebererkrankung (NAFLD). Es sind sowohl gutartige als auch progressive Verlaufsformen der NAFLD bekannt. Als Nicht-alkoholbedingte Steatohepatitis (NASH) bezeichnet man eine aggressive Unterform der NAFLD, die zu chronischer Entzündung mit resultierender Leberfibrose führen kann (Wabitsch und Kunze 2015). Von der NASH sind etwa 7 - 10 \% der übergewichtigen Kinder und Jugendlichen betroffen (AGA 2011).

Schließlich kommt es bei etwa einem Fünftel der adipösen Mädchen nach Pubertätsbeginn zur Ausbildung eines polyzystischen Ovar-Syndroms (PCO) (AGA 2011; WHO 2000).

Den Symptomkomplex aus viszeraler (also intraabdomineller) Adipositas, arterieller Hypertonie, Insulinresistenz und Typ 2-Diabetes, Dyslipidämie sowie fakultativ NASH, Hyperurikämie und PCO bezeichnet man beim Erwachsenen als „metabolisches Syndrom“ (bei Kindern existiert noch keine einheitliche Definition). Es ist assoziiert mit einem erhöhten Risiko für Atherosklerose und Typ 2-Diabetes (Wabitsch und Kunze 2015).

Weiterhin kann es zu orthopädischen Komplikationen kommen, wobei genaue Inzidenzzahlen nicht bekannt sind (AGA 2011). Beispielsweise besteht eine Assoziation zum Genu valgum und zum Morbus Blount, einer durch Gewichtsbelastung ausgelösten aseptischen Osteochondronekrose der Tibia (Wabitsch 2000; Wabitsch et al. 2013; WHO 2000). Beide können im Verlauf in einer Gonarthrose resultieren. Ebenso entwickelt sich eine Epiphysiolysis capitis femoris überproportional häufig bei übergewichtigen Kindern und kann eine Coxarthrose zur Folge haben (Strauss 1999; Wabitsch 2000).

Auch respiratorische Komplikationen wie obstruktive und restriktive Ventilationsstörungen sowie die Entwicklung eines Schlaf-Apnoe-Syndroms sind bekannt (Wabitsch et al. 2013).

Daneben existieren noch zahlreiche weitere Komorbiditäten wie eine Herabsetzung des Immunsystems mit Infektneigung und Hautveränderungen wie Striae distensae, die nicht alle im Einzelnen aufgezählt werden (Wabitsch 2000).

Kindliche Adipositas ist außerdem mit zahlreichen Komplikationen im Erwachsenenalter assoziiert. Hierzu zählt z. B. ein erhöhtes Risiko für eine koronare Herzerkrankung und eine generell erhöhte Mortalität (Baker et al. 2008; Mossberg 1989). Allerdings gibt es Hinweise, dass diese Zusammenhänge auf einer Übertragung ins Erwachsenenalter beruhen und nicht auf der kindlichen Adipositas per se (Lloyd et al. 2010). Tatsächlich scheint eine Gewichtsnormalisierung im Kindesalter auch zu einer weitgehenden Normalisierung des Risikoprofils zu führen (Korsten-Reck et al. 1994; Wabitsch et al. 1994). In diesem Zusammenhang sind Ansätze für eine erfolgreiche Therapie bereits in der Kindheit von großer Bedeutung, sowohl in Hinblick auf die individuelle Gesundheit als auch auf mögliche ökonomische Auswirkungen auf das Gesundheitssystem.

\subsection{Adipositastherapie}

\subsubsection{Therapieansätze}

\subsubsection{Indikationsstellung}

Laut den Leitlinien der AGA sollte jedem adipösen Kind der Zugang zu einer Adipositastherapie ermöglicht werden (Wabitsch und Kunze 2015). Im Sinne einer präventiven Maßnahme sollte 
auch bei (noch) nicht adipösen Kindern ohne Komorbiditäten eine Gewichtsstabilisierung angestrebt werden. Die Beurteilung der individuellen Gewichtsentwicklung sollte mithilfe des BMI-SDS erfolgen. Die Empfehlungen zur Therapieindikationen und -zielen sind im folgendem Flussdiagramm zusammengefasst (aus: Wabitsch und Kunze 2015):

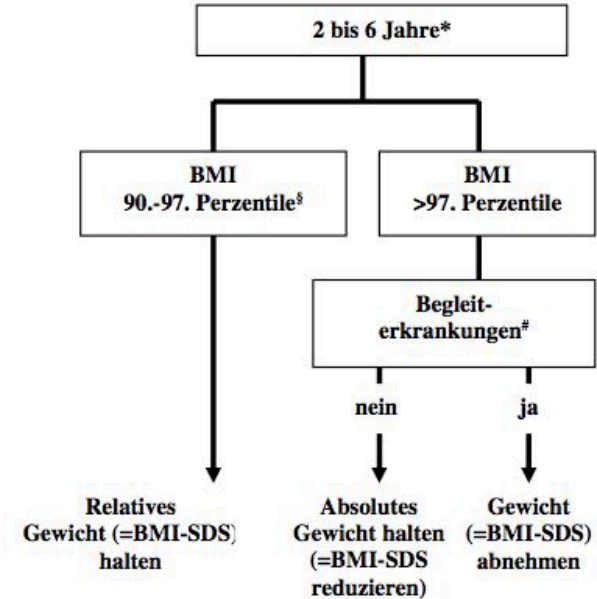

* Kinder, die jünger als 2 Jahre sind, sollten an einen Spezialisten in einem pädiatrischen Zentrum zur
Überprüfung der Indikation und zur Durchführung der Therapie verwiesen werden. Aufgrund des geringen
langfristigen Risikos sollte bei Kindern, die jünger als 4 Jahre sind und deren Eltern nicht adipös sind, zunächst
nur eine präventive Beratung durchgeführt werden.

Abbildung 4: Flussdiagramm mit Darstellung des jeweiligen Therapieziels in Abhängigkeit vom jeweiligen Alter, BMIPerzentil und Bestehen von Begleiterkrankungen

Zusätzlich ist die Indikationsstellung für eine Adipositastherapie auch abhängig vom Vorhandensein von Risikofaktoren. Hierzu zählen das Alter des Patienten und das Vorliegen einer extremen Adipositas, da höhere Grade von Adipositas zu einem höheren Lebensalter eine Übertragung ins Erwachsenenalter begünstigen (WHO 2000). Ebenfalls erfasst werden, sollten Familiensituation, Vorhandensein und Art von Komorbiditäten sowie Motivation und kognitive Fähigkeiten von Patient und Familie. Das Vorliegen einer sekundären Adipositas sollte ausgeschlossen werden. Etwaige Begleiterkrankungen werden i. d. R. nur in schwerwiegenden Ausnahmefällen therapiert, da eine relative Gewichtsabnahme meist schon eine Besserung herbeiführt (Barlow 2007; Wabitsch und Kunze 2015).

\subsubsection{Ziele und Ansätze der Adipositastherapie}

Sollte eine Adipositastherapie indiziert sein, sind dabei mehrere Ziele anzustreben. Hierzu gehören die langfristige Gewichtsreduktion mit dauerhafter Etablierung gesundheitsfördernder Verhaltensweisen sowie eine Verbesserung von Komorbiditäten, um eine normale physische und psychische Entwicklung sicherzustellen (Wabitsch und Kunze 2015).

Die Ansatzpunkte für eine solche Therapie orientieren sich an den Ursachen von Übergewicht und Adipositas, im Vordergrund stehen eine Änderung von Essverhalten und körperlicher Aktivität. Bei nicht ausreichender Studienlage sind noch nicht alle der heutigen Therapieempfehlungen evidenzbasiert. Entsprechende Empfehlungen sind deshalb gemäß der Einteilung der zugrunde liegenden Quelle gekennzeichnet: Die AGA nimmt eine Einteilung in die Evidenzklassen („EK“) I - IV vor (mit abnehmender Aussagekraft) (Wabitsch und Kunze 2015; Wabitsch und Moß 2009). Ebenfalls Grundlage dieses Abschnitts ist die Analyse eines amerikanischen Expertenkomitees, das 2005 von der American Medical Association zum Thema der Adipositastherapie ins Leben gerufen wurde (Barlow 2007). Hier wird eine Einteilung in „consistent evidence“ („CE“, verschiedene Studien kommen zum gleichen Ergebnis), „mixed evidence“ („ME“, nur ein Teil der Studien kommt zu diesem Ergebnis) vorgenommen. 
In Bezug auf das Essverhalten wird eine Reduktion der Fett- bzw. Energiezufuhr empfohlen (Wabitsch und Kunze 2015). Die Energiezufuhr muss jedoch hoch genug sein um eine normale körperliche Entwicklung nicht zu gefährden. Eine Ernährungsumstellung sollte immer durch einen qualifizierten Therapeuten betreut werden (Wabitsch und Kunze 2015; WHO 2000). Ein empfohlenes Ernährungskonzept für Kinder und Jugendliche von 1 - 18 Jahren ist die "Optimierte Mischkost". Sie wurde vom Forschungsinstitut für Kinderernährung (FKE) entwickelt und entspricht den Richtwerten für die Nährstoffzufuhr der Deutschen Gesellschaft für Ernährung (Alexy et al. 2008; Kersting und Clausen 2007). Generell sollte die Energiezufuhr zu etwa $15 \%$ aus Protein, zu $30 \%$ aus Fett und zu $55 \%$ aus Kohlenhydraten bestehen. Sie sollte sich hauptsächlich aus energiefreien Getränken und pflanzlichen, zu geringerem Anteil aus tierischen und nur zu maximal $10 \%$ aus fett- und zuckerreichen Lebensmitteln wie Süßigkeiten zusammensetzen (Wabitsch und Kunze 2015). Die Zusammensetzung der Mahlzeiten kann im Alltag mithilfe eines Ampelsystems (uneingeschränkter Konsum „grüner“, mäßiger Konsum "gelber“, nur gelegentlicher Konsum „roter" Lebensmittel) oder einer Ernährungspyramide erleichtert werden ((Wabitsch und Moß 2009), EK I). Es sollte auf einen regelmäßigen Mahlzeitenrhythmus (Wabitsch und Kunze 2015), möglichst unter Einnahme von fünf bis sechs Mahlzeiten pro Woche im Rahmen der Familie (Barlow 2007) geachtet werden. Dagegen sind Reduktionsdiäten mit sehr geringer Kalorienzufuhr (z. B. Formula-Diät) aufgrund fehlender Langzeiterfolge nur in Ausnahmefällen indiziert ((Wabitsch und Kunze 2015; Wabitsch und Moß 2009), EK II). Für Kinder von bis zu einem Jahr wird eine Ernährung durch Stillen empfohlen: bis zum Alter von sechs Monaten ausschließlich, darüber hinaus zusätzlich zu fester Nahrung ((Barlow 2007), ME).

Eine Ernährungstherapie kann die Lebensmittelauswahl positiv zu beeinflussen ((Alexy et al. 2006), EK II) und zu einem gesteigerten Obst- und Gemüsekonsum bei gleichzeitig verminderter Aufnahme fett- und zuckerreicher Lebensmittel führen ((Epstein et al. 2001), EK I). Dennoch konnten verschiedene Studien zeigen, dass eine alleinige Ernährungsumstellung langfristig nur zu geringfügigen Gewichtsreduktionen führt ((Caroli und Burniat 2006; Epstein et al. 1985),EK II). Deshalb sollten zusätzliche Therapiemaßnahmen, etwa mit dem Ziel der Steigerung der körperlichen Aktivität, angestrebt werden.

In Bezug auf letzteres wird zu einer täglichen moderaten Aktivität von mindestens 60 Minuten geraten ((Barlow 2007), ME), (Saris et al. 2003), EK IV)). Empfohlene Trainingsformen sind sowohl Kraft- als auch Ausdauertraining ((Jaeschke 2006), EK IV). Die genaue Art der körperlichen Aktivität sollte sich am Grad der Adipositas und den Vorlieben des Kindes (Wabitsch und Kunze 2015) sowie am Geschlecht orientieren ((Flores 1995), EK I). Dabei wirkt sich insbesondere ein Gruppensetting positiv auf die Gewichtsentwicklung aus ((Korsten-Reck et al. 2005), EK IV). Dennoch scheint es nicht die Teilnahme an spezifischen Sportprogrammen, sondern eher die Steigerung der Alltagsaktivität bzw. die Reduktion körperlicher Inaktivität zu sein, der die größere Bedeutung zukommt ((Wabitsch und Moß 2009), EK IV), (WHO 2000)). Programme, die auf eine Verminderung körperlicher Inaktivität hinzielen, erweisen sich sogar als erfolgreicher als solche, die eine Steigerung der körperlichen Aktivität anstreben ((Epstein et al. 1995), EK I). Ein Therapieansatz in diesem Zusammenhang ist die Begrenzung der täglichen Fernsehzeit (bzw. Zeit, die vor Computern/Spielkonsolen verbracht wird) auf maximal zwei Stunden ((Barlow 2007), CE). Ebenfalls empfiehlt sich die Entfernung eines eventuell im kindlichen Schlafzimmer befindlichen Fernsehers, um das Schlafverhalten zu verbessern (Barlow 2007).

Dennoch scheint auch eine Steigerung der körperlichen Aktivität allein nicht zu einer dauerhaften Gewichtsreduktion zu führen ((Parizkova et al. 2002), EK IV). Folglich wird auch hier die Kombination mit anderen Therapiemodulen empfohlen.

In jedem Fall wird zu einer Einbeziehung des sozialen Umfelds, insbesondere der Eltern, in die Therapie geraten, da dies einen wesentlichen Einfluss auf das kindliche Verhalten hat ((Barlow 2007), CE). Die Einbindung der Eltern in eine Ernährungstherapie ist nachweislich mit einem größeren Therapieerfolg assoziiert ((Cousins et al. 1992; Gidding et al. 2006), EK II). Gleiches gilt für Programme zur Steigerung der körperlichen Aktivität ((Epstein und Valoski et al. 1990), 
EK I). Aus diesem Grund sollten im Rahmen einer kindlichen Adipositastherapie auch immer theoretische Elternschulungen stattfinden ((Wabitsch und Kunze 2015), EK IV). Insbesondere für jüngere Kinder (etwa bis zum Alter von zwölf Jahren) ist die elterliche Einbindung von Bedeutung. Danach wird mit steigendem Lebensalter das Kind zunehmend selbst zum Hauptansprechpartner ((Barlow 2007), CE). Dies liegt nicht zuletzt daran, dass sich Jugendlichen mehr Möglichkeiten bieten, eigenverantwortlich über Nahrungsaufnahme und körperliche Betätigung zu entscheiden. Die kindliche Motivation gewinnt also bei nachlassendem elterlichen Einfluss zunehmend an Bedeutung.

Jede Adipositastherapie sollte außerdem von verhaltenstherapeutischen Maßnahmen begleitet werden, um erlernte Fähigkeiten zu festigen. In diesem Zusammenhang hat es sich als sinnvoll erwiesen, sowohl Eltern als auch Kindern schon im Vorhinein von unrealistischen Vorstellungen im Bezug auf den Gewichtsverlust abzuraten. Stattdessen sollen bereits geringe Gewichtsreduktionen als Erfolg betrachtet werden (Mühlig et al. 2014; Westenhoefer 2001).

In Bezug auf das Essverhalten sollte zur Verbesserung der langfristigen Compliance eine flexible Kontrolle angestrebt werden: Bei dieser Ernährungsform werden keine absoluten Verbote für bestimmte Nahrungsmittel ausgesprochen. Stattdessen sind nach einem „Mehr oder WenigerPrinzip“ alle Lebensmittel, zum Teil allerdings in kleineren Mengen, erlaubt ((Westenhoefer 2001), EK IV). So sollen „Heißhungeranfälle“ vermieden und damit die Langzeitcompliance unterstützt werden.

Alle erreichten Verhaltensänderungen sollten durch verschiedene Verstärkungsmechanismen unterstützt werden. Insbesondere eine Kombination verschiedener Techniken, wie Stimuluskontrolle, und Belohnung/Verstärkung, kann zu nachhaltigen Verhaltensänderungen beitragen ((Mellin et al. 1987; Yin et al. 2005), EK I). Die Verhaltenstherapie sollte sich dabei nicht nur auf die Kinder, sondern auch auf die Eltern erstrecken ((McLean et al. 2003), EK IV).

\subsubsection{Aufbau einer Adipositastherapie}

In Deutschland werden verschiedene Adipositas-Therapieprogramme für Kinder und Jugendliche angeboten. Prinzipiell kann die Behandlung sowohl ambulant als auch stationär durchgeführt werden (Wabitsch et al. 2013). Das ambulante Setting ermöglicht die Umsetzung erlernter Verhaltensweisen direkt im gewohnten (auch sozialen) Umfeld und wird deshalb empfohlen (Wabitsch und Moß 2009). Zudem ist eine ambulante Therapie vergleichsweise kostengünstiger. Das stationäre Setting bietet dagegen effizientere Kontrollmöglichkeiten für Nahrungsaufnahme und Art und Dauer von körperlicher Aktivität (Burniat et al. 2006). Aus diesem Grund führt es zu einer signifikant schnelleren Gewichtsreduktion, was einen motivationsfördernden Effekt haben kann (Reinehr und de Sousa und et al. 2006). Die AGA empfiehlt deshalb eine stationäre Therapie beim Versagen ambulanter Methoden und im Falle juveniler extremer Adipositas. Im Anschluss sollte jedoch eine ambulante Weiterbetreuung erfolgen (Wabitsch und Moß 2009).

Generell sollten Programme zur Adipositastherapie möglichst langfristig angelegt werden (Wabitsch und Kunze 2015). Sie sollten sich aus den bereits genannten Modalitäten der Ernährungs-, Bewegungs- und Verhaltenstherapie zusammensetzen und die Eltern der Patienten mit einbeziehen. Es werden zwei Therapiephasen empfohlen: Die erste Phase dient der Gewichtsreduktion, während in der zweiten Phase gelernte Verhaltensweisen gefestigt werden. Auf diese Art soll ein erneuter Gewichtsanstieg im Anschluss an die Therapie vermieden werden. Mögliche spezifische Auslöser der kindlichen Adipositas sollten zu Beginn erfasst werden (Barlow 2007). Es wird dann ein individuelles Vorgehen entwickelt. Die Therapieziele sollten in kleinen, realisierbaren Schritten gewählt werden, um die PatientenCompliance zu erhalten und Erfolgserlebnisse zu fördern (Barlow 2007).

Allerdings werden die Empfehlungen der AGA nicht von allen heutigen Therapieprogrammen umgesetzt und es bestehen zum Teil recht heterogene Vorgehensweisen (Kliche und Mann 2008). Grund hierfür ist unter anderem ein Personalmangel bzw. eine unzureichende Honorierung von Adipositastherapien im Kindes- und Jugendalter (insbesondere im ambulanten 
Bereich) (Reinehr und Wabitsch 2003). Im Jahr 2008 wurde von der AGA deshalb ein Adipositas-Schulungskonzept mit Informationen zu einem sinnvollen Aufbau von kombinierten, multidisziplinären Schulungsprogrammen vorgestellt (AGA 2008). Der Inhalt entspricht den Anforderungen des Konsensuspapiers zum Thema der Ansprüche an AdipositasSchulungsprogramme, das 2004 durch die Medizinischen Spitzenverbände und das Bundesministerium für Gesundheit und Soziale Sicherung (BMGS) herausgegeben wurde (Böhler et al. 2004). Der Zugang zu einer so konzipierten Schulung soll nach den Forderungen der Leitlinie jedem übergewichtigen oder adipösen Kind mit Komorbiditäten möglich gemacht werden (Wabitsch und Kunze 2015). Eine genaue Darstellung der Indikationsstellung findet sich in Abbildung 5 (aus: Wabitsch et al. 2013):

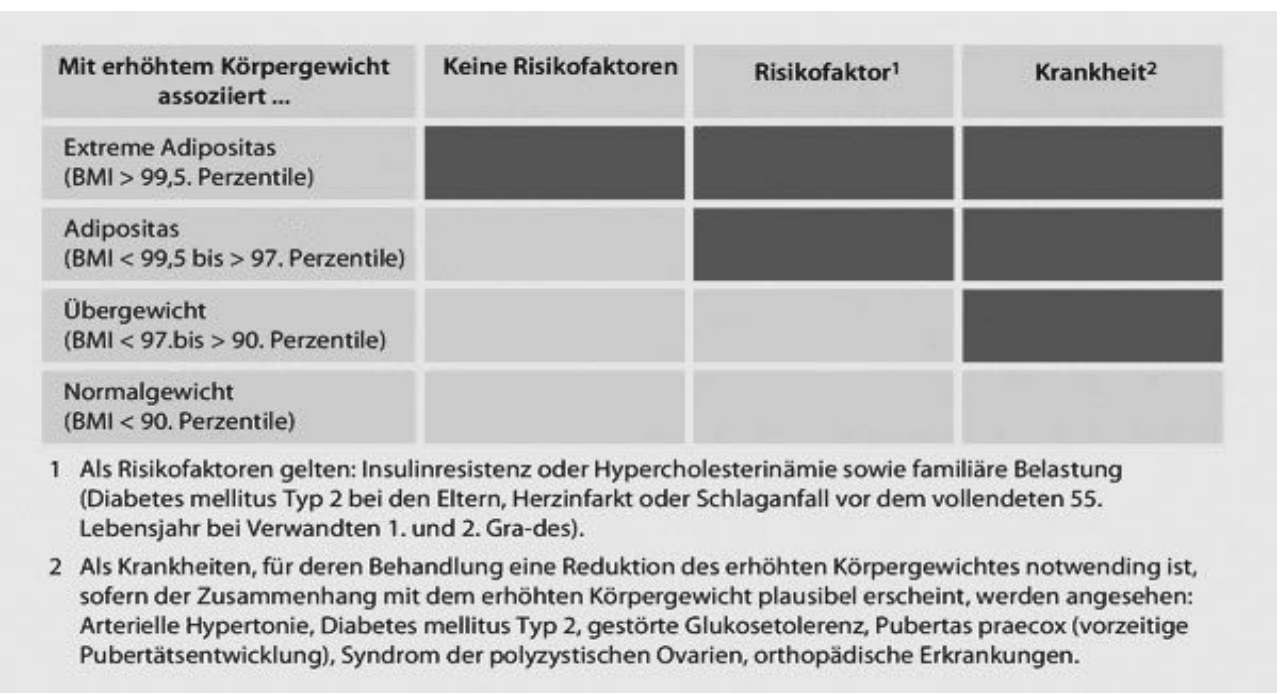

Abbildung 5: Kriterien zur Indikation übergewichtiger Kinder und Jugendlicher in eine Adipositasschulung (grau unterlegte Felder: Schulung indiziert)

Die Indikation für eine Gruppen-Schulung sollte durch den Kinder- und Jugendarzt gestellt werden. Kontraindiziert ist die Therapie bei Kindern/Familien mit mangelnder Motivation oder Gruppenfähigkeit, nicht ausreichendem Intellekt oder sekundären Adipositasformen (Wabitsch und Kunze 2015). Die Schulung erfolgt in Gruppen von Kindern möglichst ähnlichen Alters. Dabei sollen für jedes Kind individuelle Therapiepläne erstellt werden. Die Therapie muss die fünf Module „Ernährung“, „Bewegung“, „Verhaltenstherapie“, „Einbindung von Bezugspersonen“ und „Medizin“ beinhalten. Letzteres dient zur Abklärung von Ursachen und Komorbiditäten der Adipositas sowie ggf. zur Intervention (Wabitsch und Kunze 2015).

\subsubsection{Weitere Therapieoptionen}

In Einzelfällen kann auch eine medikamentöse Therapie der Adipositas in Betracht gezogen werden ((Wabitsch und Kunze 2015), EK IV). Diese sollte jedoch immer nur als adjuvante Therapie zusätzlich zu verhaltenstherapeutischen Maßnahmen etabliert werden. Mögliche Indikationen sind das Ausbleiben einer Gewichtsreduktion über neun bis zwölf Monate unter den genannten Maßnahmen sowie das Vorliegen beträchtlicher Komorbiditäten (Wabitsch et al. 2013). Insgesamt ist eine medikamentöse Therapie der Adipositas jedoch eher dem Erwachsenenalter vorbehalten und bei Kindern und Jugendlichen nicht üblich.

Sollte es trotz Einsatzes aller konservativen Therapiemethoden nicht $\mathrm{zu}$ einer Gewichtsreduktion kommen, besteht die Möglichkeit einer bariatrischen Operation ((Wabitsch und Kunze 2015), EK Ib). Aufgrund des Operationsrisikos sollte diese Option entsprechend den Empfehlungen der AGA jedoch ausschließlich in Einzelfällen bei Versagen der übrigen Therapieoptionen im Sinne einer ultima ratio-Therapie gehandelt werden (AGA 2012). Ein solcher Eingriff sollte zudem frühestens im späten Jugendalter und bei Vorliegen erheblicher Komorbiditäten in Betracht gezogen werden (Barlow 2007; Wabitsch et al. 2013). Es wird 
zwischen rein restriktiven Verfahren, die das Magenvolumen verringern, und malabsorptiven Verfahren, die die Nährstoffaufnahme im Darm reduzieren, sowie Kombinationsverfahren aus beiden Methoden unterschieden. Es existieren verschiedene Techniken, um diese Ziele umzusetzen. Beispiele für recht häufig eingesetzte restriktive Verfahren sind das Magenband ((Angrisani et al. 2005), EK III), ((O'Brien et al. 2010), EK II) sowie die Gastroplastik nach Mason (Sugerman et al. 1989). Beide bewirken eine Einengung des Mageneingangs mit konsekutiver Begrenzung der Nahrungsaufnahme. Eine Beispiel für ein Kombinationsverfahren ist die Technik des „Roux-en-Y“-Bypasses. Die Methode führt zu einer eine Umgehung eines Großteils des Magens sowie eines oralen Dünndarmabschnitts und begrenzt somit Nahrungsund Nährstoffaufnahme ((McLean et al. 2003), EK I), (Sugerman et al. 1989)).

\subsubsection{Nebenwirkungen}

Wie jede andere Behandlung kann auch eine Adipositastherapie von Nebenwirkungen begleitet sein. Zum einen besteht bei zu geringer Nährstoffaufnahme das Risiko einer Malnutrition, die die physiologische Entwicklung behindern kann (WHO 2000). Insbesondere sehr niedrigkalorische Diäten können auch mit weiteren Nebenwirkungen assoziiert sein. Beispielsweise kann es nach einer schnell erfolgten übermäßigen Gewichtsabnahme zum Auftreten eines Jojo-Effekts kommen (Wabitsch und Kunze 2015). Außerdem sind Diäten mit geringer Kalorienzufuhr mit der Entstehung von Gallensteinen assoziiert. Grund ist vermutlich eine veränderte Zusammensetzung der Gallenflüssigkeit während der Gewichtsabnahme (Johansson et al. 2014). Weiterhin besteht die Gefahr der Entwicklung von Essstörungen, wie etwa einer Binge-Eating-Problematik (Telch und Agras 1993). Schließlich kann auch die Therapie an sich durch die Konfrontation mit dem eigenen Körpergewicht zu einer psychischen Belastung führen. Dies kann sich unter anderem negativ auf das Selbstwertgefühl auswirken (Mühlig et al. 2014).

Die Möglichkeit des Bestehens solcher Nebenwirkungen sollte immer bedacht und ggf. abgeklärt werden. Sollte sich ein Verdacht bestätigen, muss eine situationsabhängige Entscheidung über die Fortführung der Therapie getroffen werden (Wabitsch und Kunze 2015).

\subsubsection{Definition von Therapieerfolg}

Es stellt sich nun die Frage, wann man eine Adipositastherapie im Kindes- und Jugendalter als erfolgreich bezeichnen kann. In entsprechenden Studien werden verschiedene Definitionen für den Therapieerfolg angewendet. Wie bereits erwähnt, sind laut Leitlinie die Ziele der Adipositastherapie eine Gewichtsstabilisierung bzw. -reduktion, eine Veränderung des Lebensstils, die Verbesserung von Komorbiditäten und die Förderung der normalen psychischen, körperlichen und sozialen Entwicklung (Wabitsch und Kunze 2015). In diesem Sinne sollte zur Beurteilung des Therapieerfolgs überprüft werden, inwieweit diese Ziele nach einer Intervention erreicht sind.

Viele Studien konzentrieren sich auf das Gewicht als Marker für den Therapieerfolg (Oude Luttikhuis et al. 2009). Als Maß zur Beurteilung der relativen Gewichtsreduktion wird der BMISDS empfohlen (Böhler et al. 2004). Im Konsensuspapier der medizinischen Spitzenverbände und des BMGS wird für Adipositasschulungen nach einem Jahr Therapie eine BMI-SDSReduktion von $\geq 0,2$ (entsprechend einer Gewichtsreduktion von $5 \%$ ) als Erfolg, von $\geq 0,5$ (entsprechend einer Gewichtsreduktion von $10 \%$ ) als sehr guter Erfolg angesehen. Dieselben Definitionen empfiehlt auch die AGA (AGA 2008). Eine BMI-SDS-Reduktion $<0,2$ wird hiernach also nicht als Erfolg betrachtet.

Eine Studie von Reinehr et al. definiert dagegen nach einem Jahr Therapie schon BMI-SDSReduktionen > 0, also jede Form der Gewichtsreduktion, als erfolgreich (Reinehr et al. 2003).

Wiegand et al. machen den Erfolg der Gewichtsreduktion zusätzlich vom weiteren Verlauf abhängig: Nach den Empfehlungen der AGA betrachten sie solche Patienten als erfolgreich, die innerhalb von bis zu einem Jahr eine BMI-SDS-Reduktion von mindestens 0,2 erreichen. Dies gilt jedoch nur unter der Voraussetzung, dass dieser BMI-SDS anschließend gehalten oder zumindest zu einem späteren Zeitpunkt (nach zwei Jahren) wieder erreicht wird (Wiegand et al. 2014). 
In den genannten Definitionen sind jedoch die übrigen von der AGA definierten Ziele der Adipositastherapie nicht inbegriffen. Man kann erwägen, dass auch eine positive Verhaltensänderung als Erfolg gewertet werden kann, selbst wenn sie nicht oder nur mit geringem Gewichtsverlust assoziiert ist (Hoffmeister et al. 2010). Auch eine Besserung von Begleiterkrankungen und kardiovaskulären Risikofaktoren kann als Erfolg angesehen werden und wird sogar als eigentlicher Nutzen der konservativen Adipositastherapie diskutiert (Holm et al. 2014; Mühlig et al. 2014). Schließlich ist auch eine Unterstützung der psychischen und sozialen Entwicklung von Bedeutung. Siegfried et al. führen beispielsweise an, dass zahlreiche Diskriminierungen bei adipösen Kindern häufig zu Schul- und Ausbildungsabbrüchen führen können. Sollte es im Rahmen der Therapie zu einer Reintegration kommen, so könnte auch dies als Therapieerfolg gewertet werden (Siegfried et al. 2006). Schließlich kann eine Verbesserung der generellen Lebensqualität erfasst und in die Therapiebewertung mit aufgenommen werden (Böhler et al. 2012).

Durch verschiedene statistische Datenanalysen werden häufig weitere Spezifizierungen des Therapieerfolgs vorgenommen, meist in Bezug auf die Gewichtsreduktion. Hierzu gehören die „Number needed to treat"- (NNT), „Intention to treat"- (ITT) und „Per Protocol“- („pP“) Analyse. Die NNT gibt an, wie viele Patienten einer Therapie zugeführt werden müssen, um bei einem zusätzlichen Patienten einen Therapieerfolg (also z. B. eine Gewichtsabnahme) herbeizuführen. Nach dem ITT-Prinzip gehen die Daten aller zuvor für die Behandlung angedachten Patienten, auch derer, die im Verlauf ausscheiden, in die Ergebnisanalyse ein. Ein „Loss-to-Follow-up“ würde so z. B. als Misserfolg gewertet werden. Im Gegensatz dazu bezieht das Per-ProtocolPrinzip für die Erfolgsanalyse nur Daten derjenigen Patienten ein, die auch die komplette angedachte Therapie erhalten haben (Böhler et al. 2013).

Anhand der zahlreichen unterschiedlichen Terminologien wird deutlich, dass momentan noch kein einheitlicher Konsens bezüglich der Definition von Therapieerfolg existiert (Böhler et al. 2004).

\subsubsection{Aktuelle Therapieangebote}

Im Folgenden sollen verschiedene aktuelle Adipositas-Therapieangebote für Kinder und Jugendliche dargestellt werden. Es existiert deutschlandweit eine Vielzahl an Schulungsprogrammen, von denen beispielhaft einige bekanntere bzw. renommiertere an dieser Stelle kurz vorgestellt werden. Bei allen folgenden Therapieangeboten handelt es sich um langfristig angelegte interdisziplinäre Gruppenschulungen.

\subsubsection{Freiburg Intervention Trial for Obese Children („FITOC“)}

Beim Programm „FITOC“ wurde 1987 in Freiburg gegründet (Korsten-Reck et al. 2002). Ziel des Programms ist eine moderate Gewichtsreduktion oder -stabilisierung, die langfristig den gewünschten Erfolg herbeiführen soll. Das Angebot richtet sich an Kinder von 8 bis 11 Jahren mit einem BMI oberhalb der 97. Perzentile. Im Rahmen einer Eingangsuntersuchung sowie weiterer Kontrolluntersuchungen erfolgt eine Erhebung von anthropometrischen, biochemischen und leistungsmedizinischen Daten. Zusätzliche Informationen werden aus Ernährungsprotokollen und Fragebögen gewonnen. Auf diese Weise wird eine regelmäßige Neuorientierung über den Therapieverlauf ermöglicht. Die Therapie selbst besteht aus regelmäßigen Sporteinheiten (dreimal wöchentlich) sowie Ernährungs- und Verhaltensschulungen. Letztere richten sich sowohl an die Patienten selbst als auch an ihre Eltern. Insgesamt finden sieben Elternabende statt, in deren Rahmen die Teilnehmer theoretische und praktische Ernährungsschulungen sowie Hintergrundinformationen zum Thema Adipositas erhalten. Weiterhin sind sieben Kinderkochnachmittage vorgesehen.

Der Therapieablauf gliedert sich in eine acht Monate andauernde Behandlungsphase sowie eine viermonatige Follow-up-Phase. 


\subsubsection{2 „Moby Dick“}

„Moby Dick“ wurde 1998 als Zielpatenschaft der Hamburger Gesundheitsbehörde mithilfe der Arbeiterwohlfart in Hamburg gegründet (Petersen und Schlesinger 2009). Das „Moby Dick“Therapieprogramm richtet sich an Kinder und Jugendliche im Alter von 8 bis 17 Jahren mit extremer Adipositas, Adipositas mit Bestehen zusätzlicher Risikofaktoren sowie Übergewicht mit Vorliegen von Begleiterkrankungen. Eine Besonderheit des Angebots ist, dass die Patienten und ihre Eltern sich im Vorhinein vertraglich zu einer regelmäßigen Teilnahme an allen vorgesehenen Stunden verpflichten. Stimmen Eltern und Patienten den Therapiebedingungen zu, erfolgt eingangs eine Erfassung der Patientendaten. Hierzu zählen Größe und Gewicht, Sozial- und Familienanamnese sowie Ess- und Freizeitverhalten.

Die Therapie beinhaltet eine theoretische und praktische Ernährungsschulung sowie ein Bewegungs- und Verhaltenstraining. Weiterhin finden Elternschulungen zu therapierelevanten Themen statt. Regelmäßige Einzel- und Gruppengespräche dienen der Reflexion des erreichten Fortschritts. Konkret ist die Therapiedauer auf 12 Monate angelegt. In dieser Zeit finden wöchentliche Gruppentreffen mit Gleichaltrigen statt. Im Rahmen dieser Treffen wird jeweils ein 1,5-stündiges Bewegungs- sowie ein ebenfalls 1,5-stündiges Verhaltens- und Ernährungstraining durchgeführt. Zusätzlich sind sechs Eltern-Kind-Nachmittage vorgesehen, in denen die Eltern ihre Kinder zu den Gruppentreffen begleiten. Daneben erhalten die Eltern verpflichtende Schulungen in Form von Fortbildungen und Elternabenden.

\subsubsection{3 „Obeldicks”}

Das Programm „Obeldicks“ wurde in Zusammenarbeit der Vestischen Kinder- und Jugendklinik Datteln mit dem Forschungsinstitut für Kinderernährung in Dortmund entwickelt (Dobe et al. 2011). Es existieren drei Untergruppen: Das Programm „Obeldicks“ richtet sich an adipöse Kinder und Jugendliche im Alter von 8 bis 16 Jahren, "Obeldicks Mini“ an adipöse Kinder im Alter von 4 bis 7 Jahren und „Obeldicks Light" an übergewichtige Kinder und Jugendliche von 8 bis 16 Jahren. Eine Besonderheit der Programme ist, dass vor Therapiebeginn eine Motivationsprüfung erfolgt und nur ausreichend motivierte Patienten auch in die Therapie eingeschlossen werden. Konkrete Voraussetzungen für eine Teilnahme sind der Ausschluss einer sekundären Adipositas, der Besuch einer Regelschule, das Ausfüllen von Fragebögen bezüglich des Ernährungs- und Bewegungsverhaltens mit Führung eines Ernährungsprotokolls über 3 Tage, der Nachweis einer sportlichen Aktivität (entweder im Rahmen der „Obeldicks“Adipositas-Sportgruppe oder im Sportverein) sowie das Vorhandensein von Komorbiditäten der Adipositas (Ausnahme: „Obeldicks Light“). Der Nachweis des Ernährungsprotokolls sowie der sportlichen Aktivität vor Therapiebeginn dient als Indikator für das Vorliegen einer ausreichenden Motivation.

An therapeutischen Disziplinen kommen eine Ernährungs-, Bewegungs- und Verhaltensschulung zum Einsatz. Zusätzlich findet eine individuelle ärztliche und psychologische Betreuung von Kindern und Eltern statt.

Der konkrete Therapieablauf gliedert sich in drei Phasen: Auf eine 3-monatige Intensivtherapie mit Gruppenschulungen in den Bereichen Ernährung und Verhalten sowie Elterninformationsveranstaltungen folgt eine 6-monatige Etablierungsphase mit individuellen psychologischen Familien- und Elterngesprächen. Zuletzt schließt sich eine 3-monatige Phase der betreuten Entlassung an. Über das komplette Behandlungsjahr besteht die Möglichkeit zur Teilnahme an wöchentlichen, 1,5-stündigen Bewegungstherapie-Einheiten. Das Programm "Obeldicks Light" unterscheidet sich von der genannten Therapie durch eine kürzere Therapiedauer von 6 Monaten, „Obeldicks Mini“ zeichnet sich durch eine Fokussierung auf die Elternschulung aus.

\subsubsection{Die „BABELUGA-Baustellen-Methode“}

Bei BABELUGA (Berliner-Adipositas-Therapie-Programm für Kinder, Jugendliche und ihre Familien - Bewegung, Beratung, Begleitung - Essen und Trinken, Eigeninitiative - Lernen, Lebensqualität - Unterstützung der Familie - Gruppentherapie für Kinder und Eltern Adipositas-Diagnostik, langfristiges Abnehmen) handelt es sich um ein Programm, das neben 
der Zertifizierung durch die AGA auch dem Konzept der Konsensusgruppe Adipositasschulung für Kinder und Jugendliche entspricht (BABELUGA, o.J.; KgAS 2011). Dieses Programm soll an dieser Stelle etwas ausführlicher vorgestellt werden, da es sich im Ansatz von den bisher abgebildeten Programmen unterscheidet. Die Besonderheit am BABELUGA-Konzept ist eine Ausrichtung der Therapie am individuellen Risiko sowie den individuellen Ressourcen eines jeden Patienten. Um dies zu ermöglichen, erfolgt zunächst eine Risikoeinschätzung anhand der Parameter „BMI“, „familiäre Situation“, „psychosoziale Problematik“, „Komorbidität der Adipositas“, „Leidensdruck“, „Veränderungsmotivation“, „Zielplanung“ und „Lebensstil“. Entsprechend den Ergebnissen werden die Patienten in vier Gruppen (A bis D) unterteilt. Jede dieser Gruppen wird nun nach einem eigenen Konzept therapiert, das an ihre jeweiligen Voraussetzungen angepasst ist. In Rahmen einer „Behandlungskette“ kommen verschiedene Therapiemodule zum Einsatz, die gleich den Gliedern einer Kette beliebig miteinander kombiniert werden können (BABELUGA o.J. ;Ernst und Wiegand 2010). Eine Darstellung der Gruppenmerkmale und der entsprechenden Therapiekonzepte findet sich in den Abbildungen 6 und 7.

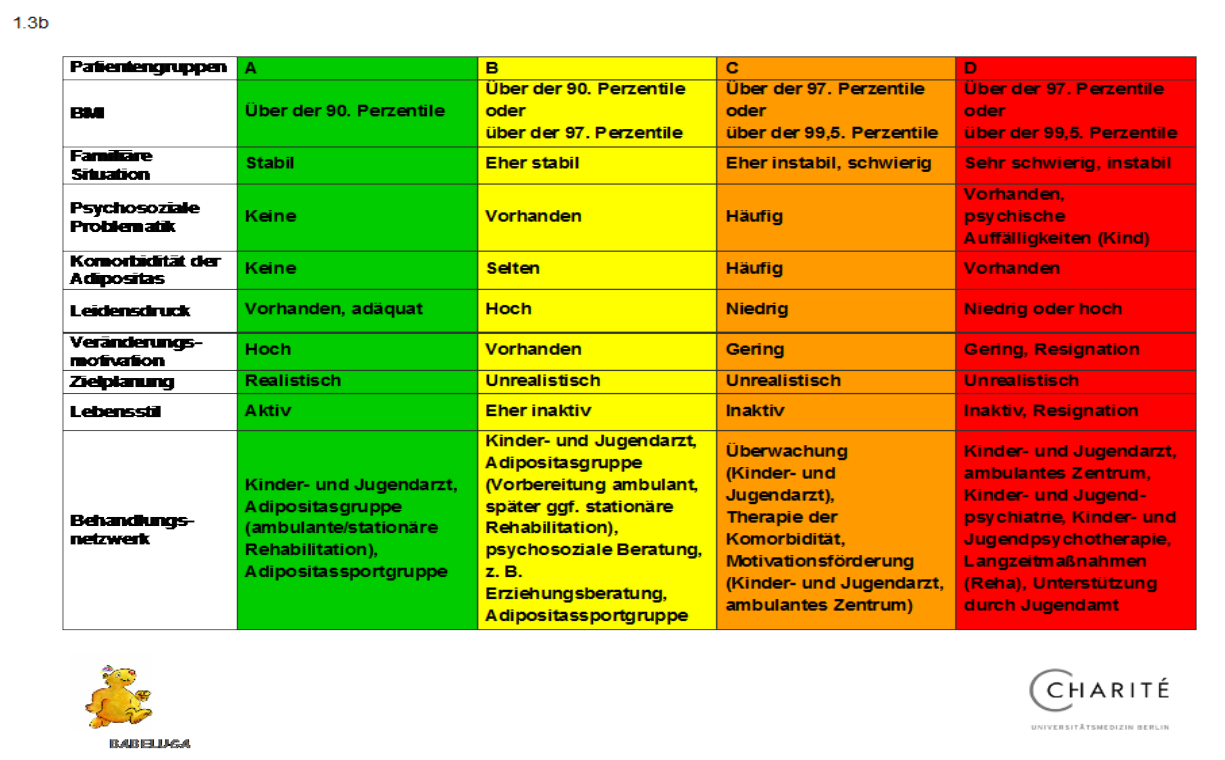

Abbildung 6: Patienteneinteilung entsprechend der BABELUGA-Methode in die Gruppen A-D anhand des erhobenen Risikoprofils mit Beschreibung des jeweiligen Therapievorgehens (aus: BABELUGA, o.J.) 


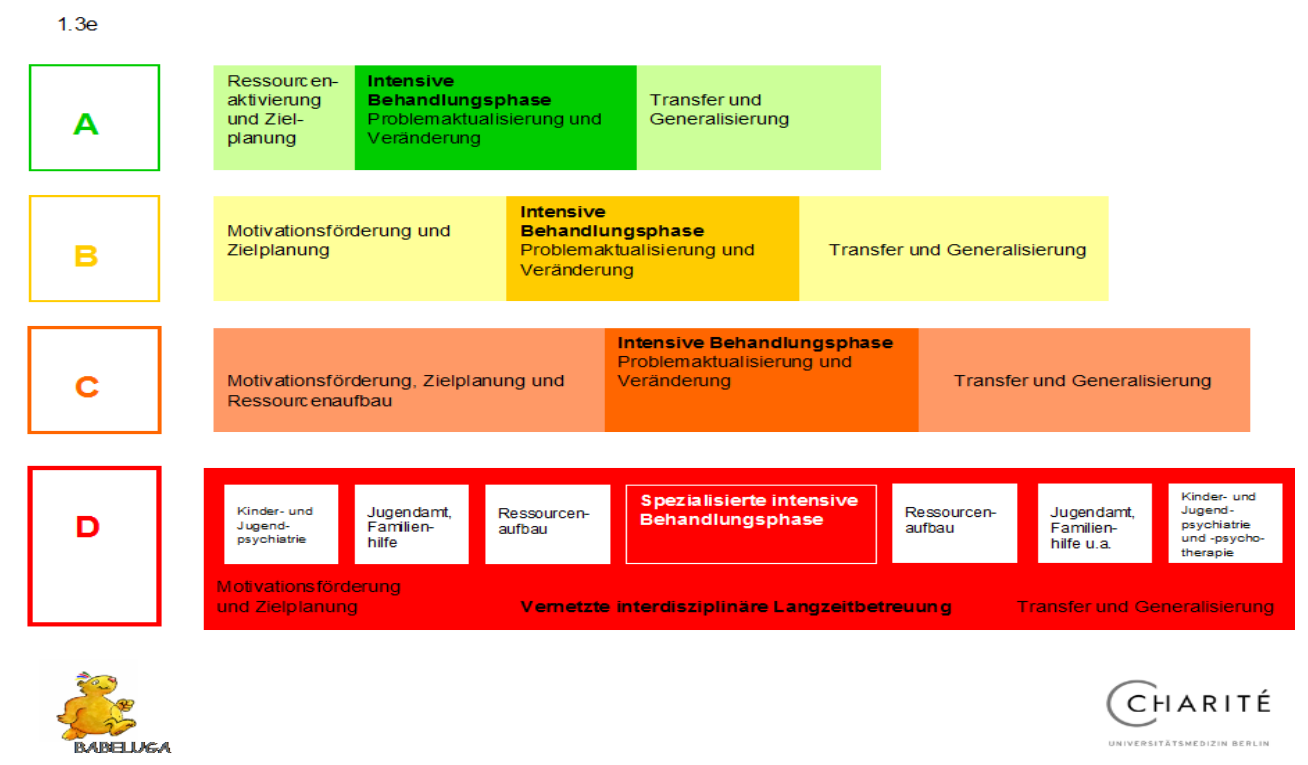

Abbildung 7: Schematisches Konzept der eingesetzten Therapiemodalitäten entsprechend der Gruppeneinteilung im Rahmen der BABELUGA-Methode. Dabei ist neben den zum Einsatz kommenden Modalitäten auch die jeweilig angedachte Therapiedauer dargestellt (aus: BABELUGA, o.J.)

Es fällt auf, dass die Zunahme ungünstiger Therapiebedingungen von Gruppe A nach Gruppe D mit einer Erhöhung der beteiligten Therapiemodule sowie einer Verlängerung der Therapiedauer einhergeht. Grundsätzlich ist die Therapie in 3 Phasen gegliedert: Die erste Phase dient der Zielplanung. Werden Patientenmotivation- und -ressourcen gering eingeschätzt, kann in dieser Phase eine entsprechende Förderung stattfinden. Als zweites schließt sich eine intensive Behandlungsphase an. Zuletzt folgt eine „Transfer- und Generalisierungsphase“ mit dem Ziel einer langfristigen Aufrechterhaltung der gelernten Fähigkeiten. Insbesondere in Gruppe D ist jedoch der Einbau zusätzlicher Module mit dem Ziel der psychosozialen Unterstützung (z. B. in Form eines Familienhelfers oder einer kinder- und jugendpsychiatrischen Betreuung) vorgesehen.

Es kommen eine ärztliche und psychologische Betreuung sowie eine Ernährungs- und Bewegungstherapie zum Einsatz (BABELUGA o.J.;Ernst und Wiegand 2010). Die Therapie innerhalb dieser Bereiche richtet sich nach dem BABELUGA-Baustellen-Prinzip. Dieses umfasst eine Evaluation von 9 Lebensbereichen (Getränke; Portionsgröße und Essmenge; Mahlzeitenhäufigkeit, Zwischendurchessen; Lebensmittelauswahl; Bewegung im Alltag; regelmäßiger Sport; Fernsehen und Computer; Süßigkeiten, Fastfood, Snacks sowie ein zusätzlicher individuell gewählter Lebensbereich). In jedem Therapiemodul erfolgt für jede dieser „Baustellen“ eine Beurteilung bezüglich der individuellen Bedeutung für den Patienten. Auf diese Art können Etappenziele festgelegt und Erfolge reflektiert werden.

\subsubsection{Erfolg und Langzeitverläufe von Therapieangeboten}

Es stellt sich nun die Frage, wie erfolgreich heutige Interventionen anhand der oben genannten Definitionen sind. $\mathrm{Zu}$ diesem Thema existieren diverse Studien. Insbesondere liegen verschiedene Metaanalysen vor, die die Erfolge unterschiedlicher Angebote zusammenfassen und vergleichen. Einige besonders repräsentative Ergebnisse werden im Folgenden besprochen. Dabei ist auch die Frage nach der Beständigkeit von Therapieerfolgen von Bedeutung: Es wird angenommen, dass viele der heutigen Angebote zwar kurzfristig, jedoch nicht auf lange Sicht effektiv sind (Oude Luttikhuis et al. 2009).

Im Jahr 2009 publizierten Luttikhuis et al. eine Cochrane-Metaanalyse unter Einschluss von 64 kontrollierten, randomisierten Studien mit einem Follow-up-Zeitraum von mindestens sechs Monaten (Oude Luttikhuis et al. 2009). Dabei wurden Studien mit Fokus auf 
verhaltenstherapeutische, medikamentöse und Maßnahmen zur Erhöhung der körperlichen Aktivität erfasst. Diese unterschieden sich sowohl im Aufbau als auch in ihrer Effektstärke. In den meisten Studien wurde eine Reduktion des Gewichts bis zum Therapieende bzw. Follow-upZeitpunkt erfasst. Unter Zusammenschau aller Ergebnisse konnte im Vergleich zu den Kontrollgruppen eine signifikante und klinisch bedeutsame Reduktion des Körpergewichts erreicht werden. Dennoch fielen die erreichten BMI-SDS-Reduktionen eher gering aus, beispielsweise mit Effektstärken von -0,04 bzw. -0,14 BMI-SDS bei Kindern bzw. Jugendlichen (Mühlig et al. 2014).

An diese Cochrane-Studie, die Daten bis Mai 2008 berücksichtigt, schlossen Mühlig et al. nahtlos eine weitere Metaanalyse mit zwischen Mai 2008 bis Dezember 2013 publizierten Studien an (Mühlig et al. 2014). Mit dem Ziel der Aktualisierung des Forschungsstandes zur Effektivität von konservativen Adipositasbehandlungen wurden 48 randomisierte, kontrollierte Studien mit insgesamt 5.025 Teilnehmern untersucht. Auch hier war ein Kriterium eine Nachbeobachtungszeit von mindestens 6 Monaten, um Aussagen zur postinterventionellen Entwicklung machen zu können. Insgesamt lag die erreichte BMI-SDS-Reduktion zwischen 0,05 und 0,42 innerhalb von 12 bis 24 Monaten nach Behandlungsbeginn. Acht Studien erfüllten alle vorgesehenen qualitativen Anforderungen (z. B. Angabe der Drop-Out-Raten, erwünschte Therapiebausteine etc.). Hier konnte nach einem Jahr eine SDS-Reduktion zwischen 0,05 und 0,39, nach zwei Jahren zwischen 0,08 und 0,42 verzeichnet werden. Eine Gewichtsnormalisierung im Rahmen einer Adipositastherapie zeigt sich anhand dieser Ergebnisse eher unrealistisch, eine Unterschreitung der Adipositasgrenze scheint nur wahrscheinlich, wenn die Ausgangswerte knapp über der Adipositasschwelle liegen. Eine Verbesserung der Komorbiditäten konnte dagegen schon bei geringer bis moderater Gewichtsreduktion erfasst werden. Vereinzelt zeigte sich ebenfalls kurzfristig eine Verbesserung der Lebensqualität.

Die Drop-Out-Raten lagen in 27 Studien bei mindestens 10 \%, in 9 bei mindestens $25 \%$. Zudem liegen sehr hohe Loss-to-Follow-up-Raten von $71 \%$ vor.

Im Jahr 2014 veröffentlichten Wiegand et al. ebenfalls eine retrospektive Studie bezüglich des Therapieerfolgs sowie dessen Prädiktoren im Rahmen von Adipositastherapieangeboten im Kindes- und Jugendalter. Bei dieser handelt es sich zwar nicht um eine Metaanalyse, aufgrund eines großen Datensatzes von 29.181 Patienten aus 157 spezialisierten Einrichtungen soll sie an dieser Stelle dennoch vorgestellt werden (Wiegand et al. 2014). Die in diese Studie eingegangenen Daten wurden aus der Adipositas-Patienten-Verlaufsdokumentation-Datenbank (APV) entnommen. In dieser Datenbank werden zu Zwecken der Qualitätssicherung Daten von Adipositastherapieprogrammen im Kindes- und Jugendalter aus Deutschland, Österreich und der Schweiz gespeichert (Reinehr et al. 2004). Wiegand et al. definierten, wie bereits im Abschnitt „Definition von Therapieerfolg“ beschrieben, solche Patienten als erfolgreich, die innerhalb von sechs bzw. zwölf Monaten eine BMI-SDS-Reduktion von mindestens 0,2 erreichten und diese bis zum Follow-up-Zeitpunkt nach zwei Jahren aufrechterhalten konnten. Ebenfalls als Erfolg wurde eine entsprechende Reduktion innerhalb von 6 Monaten gewertet, die sich zum zwei-Jahres-Follow-up wieder erfassen ließ, auch wenn es zwischenzeitlich zu einem Gewichtsanstieg gekommen war. Nach dieser Definition konnten 47,1\% der Patienten auch nach zwei Jahren noch eine ausreichende Gewichtsreduktion vorweisen. Die höchsten Reduktionswerte erreichten dabei diejenigen Patienten, die schon innerhalb von 6 Monaten eine BMI-SDS-Reduktion von 0,2 erzielen konnten. Es muss jedoch hinzugefügt werden, dass nur für 3.135 Patienten zwei-Jahres-Follow-up-Werte vorlagen, damit also sehr hohe Loss-to-Followup-Raten von $89 \%$ vorliegen.

Die Untersuchung des Erfolgs von Therapieangeboten war ebenfalls Thema der Beobachtungsstudie „Evaluation der Adipositastherapie bei Kindern und Jugendlichen in Deutschland“ („EvAKuJ“). Dabei handelt es sich um eine von der Bundeszentrale für gesundheitliche Aufklärung in Auftrag gegebene Längsschnittstudie mit dem Ziel einer Evaluation von Therapieangeboten anhand einer Kohorte übergewichtiger und adipöser Kinder 
und Jugendlicher (Böhler et al. 2012). Auch auf diese Studie soll an dieser Stelle eingegangen werden, da sie Daten von 48 Therapieeinrichtungen mit insgesamt 1916 Patienten einschließt und explizit auf postinterventionelle Verläufe eingeht. Zudem wurde auch die Entwicklung von adipogenem Verhalten, Komorbiditäten, psychischen Auffälligkeiten und Lebensqualität untersucht.

Die Patienten wurden jeweils zu Therapiebeginn, Therapieende („,kurzfristig“) und zum Followup-Zeitpunkt ein („mittelfristig“) und zwei („langfristig“) Jahre nach Therapieende untersucht.

Zum Zeitpunkt des Therapieendes ergaben sich im Rahmen einer ITT-Analyse laut Böhler et al. recht gute Effektstärken. Bei einer Erfolgsdefinition als BMI-SDS-Reduktion um mindestens 0,2 erreichten $56 \%$ der Patienten eine ausreichende Gewichtsreduktion. Dabei zeigten sich die stationären Programme mit ITT-Erfolgsraten von $80 \%$ erfolgreicher als die ambulanten mit Raten von $35 \%$. Langfristig ergab sich dagegen eine vergleichsweise niedrige Erfolgsrate: Nach einem Jahr waren im Rahmen einer ITT-Analyse nur noch $13 \%$, nach zwei Jahren $14 \%$ der Patienten erfolgreich. Dabei zeigte das stationäre Setting nach zwei Jahren ITT-Erfolgsraten von $7 \%$, während das ambulante Setting zumindest $17 \%$ erreichte. Während also kurzfristig eine stationäre Behandlung effektiver war, erzielte eine ambulante Therapie auf lange Sicht größere Erfolge. Auch bei Anwendung einer per Protocol-Analyse erreichten langfristig nur $50 \%$ der ambulanten und $37 \%$ der stationären Patienten einen ausreichenden Therapieeffekt. Diese Ergebnisse untermauern die Einschätzung, dass kurzfristige Erfolge zwar möglich sind, jedoch selten langfristig anhalten.

In Bezug auf adipogenes Verhalten zeigten sich kurzfristig positive Veränderungen. So ließ sich direkt im Anschluss an die Therapie eine Verbesserung der Lebensmittelauswahl bei $80 \%$ der Patienten nachweisen (nach ITT). Ebenso konnte zum Therapieende eine Zunahme des Sportund Bewegungsverhaltens bei $40 \%$ der Patienten (nach ITT) erfasst werden, allerdings ohne Reduktion sitzender Tätigkeiten wie TV-Konsum oder Computer-Nutzung. Dennoch konnten diese positiven Entwicklungen mittel- und langfristig nicht mehr dargestellt werden. Demnach scheint es durch die eingegangenen Therapieprogramme nicht zu einer langfristigen Verbesserung Adipositas-bezogener Verhaltensweisen zu kommen.

Bei Untersuchung der Komorbiditäten konnte kurzfristig eine deutliche Verbesserung kardiovaskulärer Risikofaktoren, besonders des Blutdrucks, festgestellt werden. Aufgrund nicht ausreichender Daten ist die langfristige Entwicklung jedoch unklar.

In Bezug auf die psychische Entwicklung konnte kurzfristig kein Effekt, nach zwei Jahren jedoch bei etwa der Hälfte der stationär behandelten und zwei Drittel der ambulant behandelten Teilnehmer eine Verbesserung von psychischen Auffälligkeiten gesehen werden (pP-Analyse). Schließlich konnte kurzfristig ebenfalls eine Verbesserung der Lebensqualität erfasst werden.

Es ist jedoch hinzuzufügen, dass die oben genannten ITT-Auswertungen aufgrund sehr hoher Loss-to-Follow-up-Raten (stationär $81 \%$, ambulant 66 \%) und einer Drop-Out-Rate von $10 \%$ nur beschränkt valide sind.

Insgesamt zeigen sich die Erfolge von Therapieprogrammen vor allem auf lange Sicht eher begrenzt. Maximal 50 \% der Patienten, in der Regel jedoch weniger, können auch langfristig eine ausreichende Gewichtsreduktion aufrechterhalten. In diesem Zusammenhang stellt sich die Frage, welche Voraussetzungen den Erfolg einer Adipositastherapie im Kindes- und Jugendalter beeinflussen und begünstigen können.

\subsubsection{Prädiktoren für den Therapieerfolg}

Warum manche Kinder von Interventionen profitieren, andere jedoch nicht, ist momentan noch weitgehend unklar. Einige Prädiktoren, die mit dem Therapieerfolg bzw. -misserfolg assoziiert sind, sind jedoch bekannt.

$\mathrm{Zu}$ diesen Prädiktoren gehören zum einen die schon genannten Therapiebausteine, die eine erfolgreiche Therapie begünstigen. Hierunter fallen der multidisziplinäre Therapieaufbau und die Einbeziehung von Eltern und sozialem Umfeld in die Therapie (Epstein und McCurley et al. 1990; Epstein et al. 1994). 
Daneben konnten jedoch noch weitere Faktoren ausgemacht werden, von denen einige allerdings im Rahmen der Therapie nicht beeinflussbar sind. Hierzu gehört beispielsweise das Geschlecht der Patienten. So konnten Wiegand et al. langfristig eine erfolgreichere Gewichtsreduktion bei Jungen im Vergleich zu Mädchen beobachten (Wiegand et al. 2014). Dieses Ergebnis ließ sich jedoch in anderen Untersuchungen nicht nachweisen (Braet 2006; Reinehr et al. 2003).

Auch das Alter bei Therapiebeginn zeigt eine Assoziationen zum Therapieerfolg. In mehreren Untersuchungen konnte eine negative Korrelation zwischen Alter und langfristiger Gewichtsreduktion dargestellt werden (Reinehr et al. 2010; Reinehr et al. 2009; Wiegand et al. 2014). Eine mögliche Erklärung für dieses Phänomen ist die leichtere Beinflussbarkeit adipogener Verhaltensweisen bei Kindern im Vergleich zu Jugendlichen (Wiegand et al. 2014). Auch das Ausgangsgewicht scheint sich auf die Gewichtsentwicklung auszuwirken. So konnten Böhler et al. beispielsweise bessere Therapieerfolge bei Patienten mit niedrigerem Ausgangsgewicht erfassen (Böhler et al. 2012).

Dennoch lassen sich die genannten Assoziationen des Therapieeffekts zu Geschlecht, Alter und Ausgangsgewicht nicht in allen Studien nachvollziehen (Braet 2006; Reinehr et al. 2003).

Neben dem Ausgangsgewicht scheint sich auch ein hoher initialer relativer Gewichtsverlust im Rahmen der Therapie positiv auf den Behandlungserfolg auszuwirken und ein Prädiktor für den Gewichtsverlust ein Jahr nach Therapiebeginn zu sein (Epstein et al. 1994). Ein Erklärungsansatz hierfür ist, dass hohe Gewichtsverluste gleich zu Beginn der Therapie mit einer Steigerung der Motivation einhergehen können (Braet 2006).

Ebenso scheinen familiäre Faktoren einen Einfluss auf die Effektstärke zu haben. Hierzu gehört zum einen das elterliche Gewicht: Ein niedrigeres Elterngewicht ist mit einem größeren kindlichen Gewichtsverlust assoziiert (Epstein et al. 1994; Fröhlich et al. 2011). Im Hinblick auf die Entwicklung kindlicher Adipositas im Rahmen eines Zusammenspiels von genetischen Faktoren und Umweltfaktoren kann ein niedrigeres Elterngewicht hinsichtlich beider Aspekte positive Auswirkungen auf die Erfolgschancen in der Gewichtsreduktion haben (Epstein und Wing 1987). Ein weiterer Faktor ist der familiäre SES. Es handelt sich dabei um einen Positivprädiktor: Ein hoher SES ist auch mit einer größeren relativen Gewichtsreduktion assoziiert (Röbl et al. 2013), wenngleich auch dies nicht für alle Untersuchungen gilt (Reinehr et al. 2003). Ein möglicher Grund ist, dass ein niedrigeres Einkommen mit einer größeren Familie assoziiert ist. Beide Faktoren können die Zeit beeinflussen, die auf das Essverhalten eines Kindes verwendet werden kann. Tatsächlich zeigte sich die Familiengröße in einer Studie von Epstein et al. als besserer Prädiktor für die Gewichtsreduktion nach einem Jahr als das Gewicht der Eltern (Epstein et al. 1988).

Weiterhin gibt es Hinweise für Korrelationen zwischen einer alleinerziehenden Familiensituation und einem geringeren Gewichtsverlust (Reinehr et al. 2003).

Auch familiäre Dysfunktionen können sich negativ auf den Therapieerfolg auswirken. Kirschenbaum et al konnten zeigen, dass sich sowohl eine besonders liberale, antiautoritäre als auch eine "chaotische", dysfunktionale Familiensituation negativ auf den Behandlungserfolg auswirken (Kirschenbaum et al. 1984). Ein möglicher Grund ist ein Mangel an Organisationsfähigkeit, welche einen wesentlichen Faktor zum Erreichen gesetzter Ziele darstellt.

Weiterhin spielen psychische Faktoren eine Rolle für die Gewichtsentwicklung. So ist in einer Studie von Epstein et al. die Entwicklung psychischer Störungen im Rahmen des Follow-up mit einer schlechteren langfristigen Gewichtskontrolle assoziiert (Epstein et al. 1994). In diesem Zusammenhang korreliert auch das Auftreten von Essstörungen mit geringeren Behandlungseffekten (Braet 2006).

Schließlich spielen auch biologische Faktoren eine Rolle. So konnte z. B. gezeigt werden, dass höhere Leptin- und niedrigere Ghrelin-Plasma-Spiegel mit einer erneuten Gewichtszunahme nach initialem Gewichtsverlust zusammenhängen. Da höhere Leptinwerte jedoch gewöhnlich ein Sättigungs- und hohe Ghrelinspiegel ein Hungergefühl verursachen, könnte diesem Phänomen eine verminderte Empfindlichkeit für beide Hormone bei den entsprechenden Patienten zugrunde liegen (Crujeiras et al. 2010). 
Nicht zuletzt stellt auch die Motivation des Patienten einen wichtigen Einflussfaktor auf den Therapieerfolg dar (Reinehr et al. 2003). Allerdings konnten bis heute noch keine zufriedenstellenden Methoden zur Erfassung der Patientenmotivation entwickelt werden. Verschiedene Ansätze sind momentan in der Diskussion. Hierzu zählen etwa die Selbstzahlung der Therapie durch den Patienten, die Anzahl vorheriger Diätversuche oder eine Erfassung im Rahmen von psychologischen Testverfahren (Reinehr et al. 2003).

\subsection{Adipositas als chronische Erkrankung}

Dass es sich bei Adipositas um eine eigenständige Erkrankung handelt, ist bereits seit mehreren Jahren allgemein anerkannt. Tatsächlich kann man Adipositas im Kindes- und Jugendalter zusätzlich auch als chronische Erkrankung betrachten (Disse und Zimmer 2014). Unbestreitbar existieren Studien, die aufzeigen, dass eine dauerhafte Gewichtsreduktion mit Beibehaltung bis ins Erwachsenenalter möglich ist (Epstein et al. 1994). Dennoch entspricht ein solcher Verlauf nicht dem Regelfall, sondern eher der Ausnahme. Insgesamt liegt die Wahrscheinlichkeit einer Übertragung der Adipositas ins Erwachsenenalter bei 25 - 50 \% (Must und Strauss 1999; Whitaker et al. 1997). Betrachtet man die kindliche Adipositas also als chronische Erkrankung, stellt sich die Frage, ob sie nicht, wie andere chronische Erkrankungen auch, eine dauerhafte Behandlung notwendig macht. Nach Disse et al. machen die eher limitierten Langzeiterfolge in der kindlichen Adipositastherapie deutlich, dass heutige Interventionen regelhaft zu kurz angelegt sind (Disse und Zimmer 2014). Auch Mühlig et al. argumentieren, dass zumindest im Hinblick auf das hohe Komorbiditätsrisiko eine dauerhafte diagnostische und therapeutische Anbindung adipöser Kinder und Jugendlicher nötig ist (Mühlig et al. 2014).

In diesem Zusammenhang beschäftigt sich eine zunehmende Zahl von Studien mit der Frage nach der Effektivität von länger als üblich angelegten Interventionskonzepten. Eine Möglichkeit hierfür ist der Anschluss spezieller Aufrechterhaltungsprogramme an die eigentliche Therapie. Ein Beispiel ist eine Studie von Wilfley et al. (Wilfley et al. 2007): Im Rahmen eines kontrollierten, randomisierten Settings wurden die Teilnehmer zunächst in Gruppen eingeteilt und eine Adipositastherapie durchgeführt. Das weitere Verfahren divergierte je nach Gruppe: In der ersten Gruppe fanden Erhaltungsinterventionen mit Fokus auf verhaltenstherapeutische Aspekte statt, in der zweiten mit Fokus auf sozialen Fähigkeiten. Die dritte Gruppe diente als Kontrolle und erhielt keine weitere Behandlung. Im Ergebnis konnten die Teilnehmer der beiden Interventionsgruppen zumindest kurzfristig einen signifikant geringeren erneuten Gewichtsanstieg im Anschluss an die Therapie verzeichnen.

Einen etwas anderen Ansatz wählten Deforche et al. (2005): Ebenfalls im Rahmen einer kontrollierten, randomisierten Studie schloss sich bei der Interventionsgruppe ein fünfmonatiges Erhaltungsprogramm an eine Adipositastherapie an. Dieses bestand aus dem Führen eines Aktivitäten-Tagebuchs, welches in zweiwöchentlichen Abständen telefonisch mit einem Therapeuten besprochen wurde. Auf diese Art konnten Erfolge und Misserfolge erörtert und neue Wochenziele gesetzt werden. Im Vergleich zur Kontrollgruppe zeigte die Interventionsgruppe insgesamt ein höheres $\mathrm{Ma}$ an körperlicher Aktivität bei gleichzeitig geringerem Wiederanstieg des relativen Gewichts.

Die Ergebnisse dieser beiden Studien könnten darauf hinweisen, dass das schlichte Beenden einer Adipositastherapie eine erneute Gewichtszunahme durch Rückfall in alte Verhaltensmuster begünstigt. Ein längerfristiger angelegtes Programm könnte sich auf diesen Aspekt günstig auswirken.

\subsection{Fragestellungen dieser Arbeit}

In einer Kohorte adipöser Patienten, die im Sozialpädiatrischen Zentrum der Universitätsmedizin Göttingen im Zeitraum von 2004 bis 2012 interdisziplinär behandelt wurden, wurden die folgenden Fragestellungen untersucht: 
1. Welche Prädiktoren für eine erfolgreiche Adipositastherapie im Kindes- und Jugendalter lassen sich für das vorliegende Kollektiv erfassen?

2. Welche dieser Prädiktoren sind im Rahmen der Therapie beeinflussbar, welche sind von der Therapie unabhängig?

3. Gibt es unterschiedliche Prädiktoren für den kurzfristigen und den langfristigen Therapieerfolg?

4. Wie lässt sich eine Adipositastherapie im Kindes- und Jugendalter mithilfe der erfassten Prädiktoren optimieren? 


\section{$2 \quad$ Material und Methoden}

\subsection{Ethikvotum}

Die im Folgenden beschriebene Studie wurde durch die Ethikkommission der Medizinischen Fakultät der Universität Göttingen genehmigt (Antragsnummer: 19/6/13).

\subsection{Patientenkollektiv und Studiendesign}

Bei der vorliegenden Arbeit handelt es sich um eine Kohortenstudie. Eingeschlossen wurden alle Patienten, die im Zeitraum 01.10.2004 bis 30.09.2012 am Adipositas-Behandlungsprogramm des Sozialpädiatrischen Zentrums (SPZ) der Universitätsmedizin Göttingen teilgenommen haben und zum Zeitpunkt des Therapiebeginns mindestens elf Jahre alt waren. Ein Therapeutenteam von Kinderärzten, Ernährungsberatern, Psychologen, Sozialpädagogen, Physiotherapeuten und Ergotherapeuten führte die Behandlung durch. Das therapeutische Angebot umfasste neben individuellen Einzeltherapien multidisziplinäre Einzeltherapien sowie multidisziplinäre Gruppentherapien. Die Therapien erfolgten gemäß den Leitlinien der Arbeitsgemeinschaft Adipositas im Kindes- und Jugendalter. Hierbei erfolgte die Wahl des Therapieangebotes in der Regel durch die Patienten und deren Erziehungsberechtigte.

Beginn der Datenerhebung war der 30.09.2013, sodass für die eingeschlossenen Patienten für mindestens 12 Monate keine Termine mehr in der Adipositas-Sprechstunde des SPZ stattgefunden hatten, um so den Langzeitverlauf der Gewichtsentwicklung der behandelten Patienten beurteilen zu können. Für Patienten, die nach dem ersten Arzttermin keine weiteren Termine mehr zur Adipositas-Sprechstunde im SPZ wahrgenommen hatten, wurde ein rein diagnostischer Ansatz angenommen. Zudem lagen für diese Patienten keine aktuelleren Gewichtsdaten mehr vor. Es war folglich keine Beurteilung der Gewichtsentwicklung möglich. Aus diesen Gründen gingen diese Patienten nicht in die Auswertung ein. Insgesamt wurden 358 der 442 Kinder und Jugendlichen, die in dieser Zeit die Sprechstunde besuchten, in die Auswertung aufgenommen.

Für dieses Kollektiv wurden, soweit möglich, Gewicht und Größe jeweils zu Beginn (Zeitpunkt $\mathrm{T}_{0}$ ) und Ende (Zeitpunkt $\mathrm{T}_{1}$ ) der Therapie sowie weitere Daten retrospektiv erfasst. Zusätzlich erfolgte eine weitere Datenerhebung im Sinne eines Follow-up zu einem dritten, aktuelleren Zeitpunkt (Zeitpunkt $\mathrm{T}_{2}$ ).

Den Patienten wurden hierfür zunächst auf dem Postweg standardisierte Informations- sowie Einwilligungsbögen zugesandt (siehe Anhang). Kam es innerhalb von zwei Wochen nach Eingang nicht zu einer Einwilligung bzw. Ablehnung durch den Patienten, erfolgte, wie im zugesandten Patienteninformationsbogen vermerkt, eine telefonische Kontaktaufnahme. Auf diese Art willigten 128 der 358 Patienten (35,8 \%) in die Teilnahme an der Studie ein, 75 der 358 Patienten (20,9 \%) lehnten eine Teilnahme ab. Bei Patienten, deren aktuelle Kontaktdaten dem SPZ wegen Verzugs nicht vorlagen oder die auch nach dreimaligem Versuch telefonisch nicht erreichbar waren, war im Sinne eines Loss-to-Follow-up keine weitere Datenerhebung möglich. Dies betrifft 135 der 358 Patienten (37,7 \%). Ausgenommen waren 32 Patienten (8,9 $\%$ ), bei denen aktuellere Gewichts- und Größenangaben aus Krankenakten erfasst werden konnten. Die BMI-Daten dieser Patienten gingen für den Zeitpunkt $\mathrm{T}_{2}$ ebenfalls in die Auswertung mit ein.

\subsection{Datenerhebung}

Für alle 358 Patienten erfolgte zunächst eine retrospektive Datenerhebung. Extrahiert wurden die Daten aus der Krankenakte sowie dem elektronischen krankenhausinternen Patienteninformationssystem, zugrunde gelegt wurden Aufnahmeuntersuchungen, Verlaufsbeschreibungen und Arztbriefe. Die auf diese Art erhobenen Daten für Gewicht und 
Größe basieren folglich auf im Rahmen der Sprechstunde durchgeführten Messungen. Die Kinder und Jugendlichen werden dabei standardmäßig in Unterwäsche gewogen, die Erfassung der Körpergröße erfolgt mithilfe eines Messstabs.

Die Datenerhebung nach Bereiterklärung zur Teilnahme an der Studie erfolgte mittels eines Fragebogens, welcher sich im Anhang der vorliegenden Arbeit wiederfindet. Dieser wurde den Patienten postalisch zugesandt und entweder auf ebendiesem Weg ausgefüllt zurückgesandt oder nach Einwilligung telefonisch abgefragt. Die Datenerhebung erstreckte sich über einen Zeitraum von 6 Monaten (10/13 - 04/14).

Wie bereits erwähnt wurde in Fällen, in denen auf diese Art zum Zeitpunkt $\mathrm{T}_{2}$ keine Erhebung der kindlichen Daten von Gewicht und Größe möglich gewesen war, zusätzlich eine Recherche der Krankenakte bzw. des krankenhausinternen digitalen Patienteninformationssystems vorgenommen. So konnten eventuell zu späteren Terminen (außerhalb der AdipositasSprechstunde) erfasste Angaben mit aufgenommen werden.

\subsection{Methoden der Datenanalyse}

Als Grundlage für die Datenanalyse der vorliegenden Studie dienten BMI-SDS.

Der BMI wurde, sofern die benötigten Daten (Gewicht und Körpergröße) vorlagen, für alle 358 Patienten zu den Zeitpunkten $\mathrm{T}_{0}, \mathrm{~T}_{1}$ und $\mathrm{T}_{2}$ berechnet.

Die Berechnung des BMI erfolgt durch folgende Formel:

$$
\mathrm{BMI}=\frac{\text { Masse }(\mathrm{kg})}{\text { Körpergröße }(\mathrm{m})^{2}}
$$

Zur Klassifikation des errechneten BMI-Wertes wurde dann eine Einordnung in Perzentile vorgenommen. Dabei wurden, wie in den Leitlinien der AGA empfohlen, die Perzentile nach Kromeyer-Hauschild et al. zugrunde gelegt (Kromeyer-Hauschild et al. 2001). Da die vorliegende Arbeit sich jedoch mit Langzeitverläufen in der Adipositastherapie beschäftigt, wurden auch Patienten in die Studie eingeschlossen, die zum Zeitpunkt $\mathrm{T}_{2}$ ein Alter von 18 Jahren bereits überschritten hatten. Die Referenzwerte nach Kromeyer-Hauschild et al. beziehen sich allerdings ausschließlich auf Kinder und Jugendliche, sodass für die erwachsenen Patienten Daten aus der Nationalen Verzehrstudie herangezogen wurden (Hemmelmann et al. 2010).

Die Definition von Übergewicht und Adipositas erfolgte ebenfalls anhand den Empfehlungen der Leitlinie: Kinder mit einem BMI über der 90. Perzentile der Referenzpopulation wurden als übergewichtig, über der 97. Perzentile als adipös und über der 99,5. Perzentile als extrem adipös eingestuft (Wabitsch und Kunze 2015).

Mit der Berechnung des BMI-SDS-Wertes kann nun weiterhin angegeben werden, um das Wievielfache einer Standardabweichung ein individueller BMI-Wert vom Median der Referenzgruppe abweicht. Dabei bedeutet ein BMI-SDS-Wert von +1 bzw. -1 ein Abweichen des BMI um eine Standardabweichung nach oben bzw. nach unten. Bei einem Wert von +1 liegt der BMI also im 84. Perzentil, bei einem Wert von -1 im 16. Perzentil der Referenzgruppe. Eine Abweichung um zwei Standardabweichungen nach oben bzw. unten entspricht einem BMI-SDS von +2 bzw. -2 und damit dem 97,7. bzw. 2,3. Perzentil der Referenzgruppe (KromeyerHauschild et al. 2001).

Der BMI-SDS errechnet sich mithilfe der Formel:

$$
\mathrm{SDS}_{\mathrm{LMS}}=\frac{[\mathrm{BMI} / \mathrm{M}(\mathrm{t})]^{\mathrm{L}(\mathrm{t})}-1}{\mathrm{~L}(\mathrm{t}) \mathrm{S}(\mathrm{t})}
$$

Dabei steht L (Lambda) für die Box-cox-power-Transformation, diese dient bei der Berechnung zur Stabilisierung der Varianz und damit der Standardabweichung. M (My) steht für den Median der Referenzpopulation, S (Sigma) für den Variationskoeffizenten, t für das Alter der Patienten. Die Parameter L, M und S ermöglichen nach der Methode von Cole (Cole 1990) eine Berechnung der Perzentilen mit Beschreibung der Verteilung des BMI über das Alter. Die Daten für L,M und S 
wurden wie die übrigen Referenzwerte entweder von Kromeyer-Hauschild et al. oder aus der Nationalen Verzehrstudie herangezogen (Hemmelmann et al. 2010; Kromeyer-Hauschild et al. 2001).

Es wurden, soweit Gewicht und Größe zu den jeweiligen Zeitpunkten für die jeweiligen Patienten vorlagen, die BMI-SDS-Werte zu den 3 Zeitpunkten $T_{0}, T_{1}$ und $T_{2}$ berechnet. Durch Subtraktion der jeweiligen SDS-Werte zu den verschiedenen Zeitpunkten voneinander wurden die BMI-SDS-Reduktionen als Indikator für den Therapieerfolg über drei Zeiträume errechnet. Davon erstreckt sich der erste über die Zeit der Adipositastherapie im SPZ, der zweite über die Zeit zwischen Therapieende und aktueller Kontaktaufnahme (dies entspricht dem Langzeitverlauf), der dritte über den gesamten Verlauf vom Beginn der Therapie bis zum Zeitpunkt der erneuten Kontaktaufnahme.

Für diese drei Gruppen wurde daraufhin untersucht, ob ein statistisch signifikanter Zusammenhang zu verschiedenen Parametern bestand. Der Großteil der Datenanalyse erfolgte in Form einer pP-Analyse, wobei nur diejenigen Patienten in die Auswertung eingingen, die auch die vorgesehene Therapie erhalten hatten. Der Erfolg der Gewichtsreduktion ist jedoch ebenfalls als ITT-Analyse angegeben, wobei auch zum Follow-up-Zeitpunkt nicht mehr erfasste Patienten (Loss-to-Follow-up) als Therapie-Misserfolg in die Auswertung eingehen.

\subsection{Parameter der Datenanalyse}

Wie bereits erwähnt wurde ein Teil der untersuchten Daten aus den Patientenakten bzw. dem digitalen Patienteninformationssystem entnommen. Eine Zusammenfassung der auf diese Weise erhobenen Parameter findet sich in Tabelle 1.

Tabelle 1: Untersuchte Parameter, die mithilfe von Patientenakten bzw. des krankenhausinternen elektronischen Patienteninformationssystem erhoben wurden

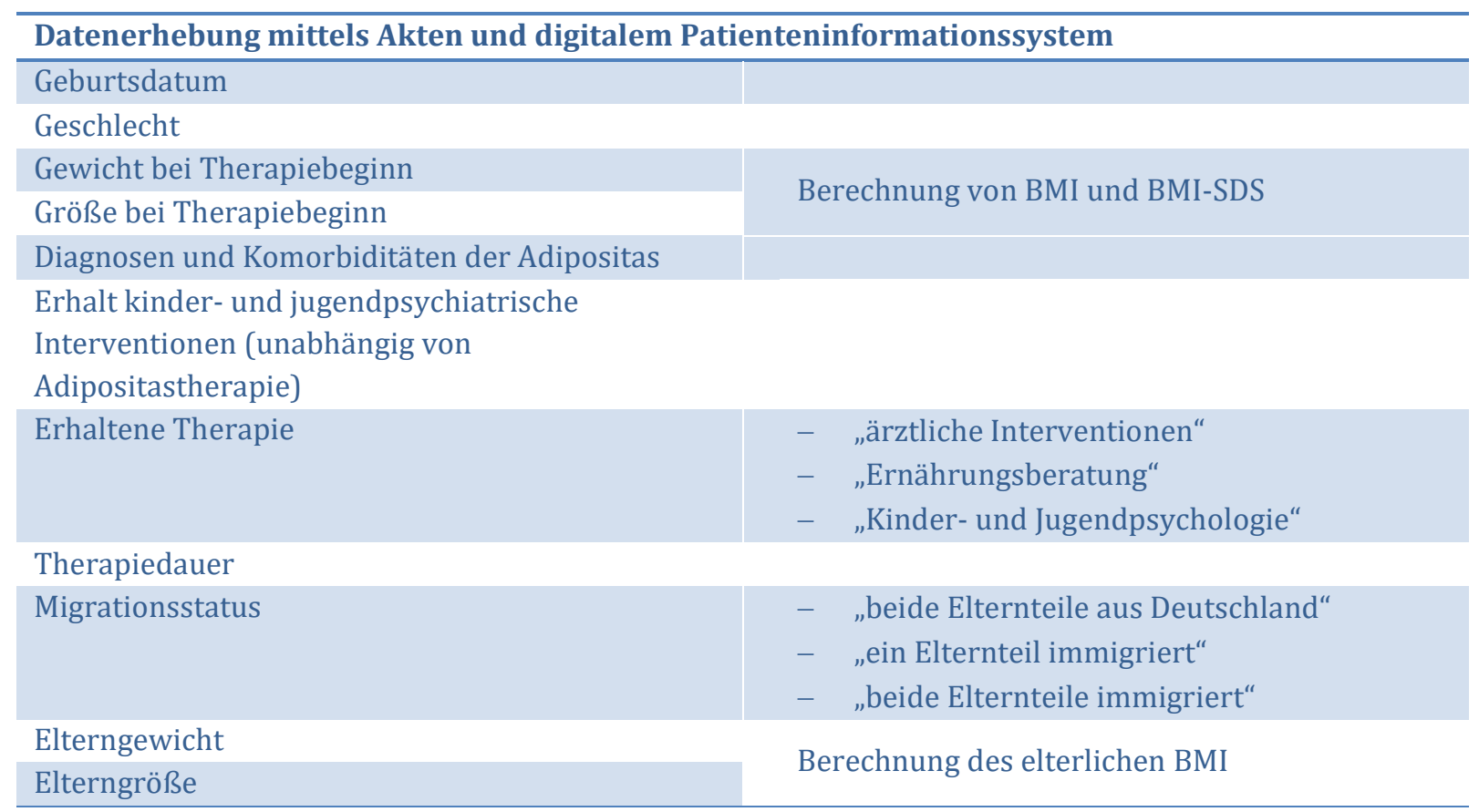

Bezüglich der Therapie wurde ein Erhalt aller drei Therapiebausteine („ärztliche Interventionen“, „Ernährungsberatung“, „Kinder- und Jugendpsychologie“) in die Kategorie „multiprofessionelle Therapie“ eingeordnet. Fanden dagegen zusätzlich zu den Arztterminen Interventionen aus nur einem weiteren Baustein statt, so wurde dies als „individuelle Therapie“ eingestuft. 
Die Datenerhebung für den Follow-up-Zeitpunkt erfolgte mithilfe des zugesandten Fragebogens (siehe Anhang) bzw. telefonisch. Die auf diese Art erhobenen Daten finden sich in Tabelle 2.

Tabelle 2: Untersuchte Parameter, die postalisch bzw. telefonisch mittels Fragebogen erfasst wurden.

\begin{tabular}{|c|c|}
\hline \multicolumn{2}{|l|}{ Datenerhebung mittels Fragebogen } \\
\hline Aktuelles Gewicht & \multirow{2}{*}{ Berechnung des aktuellen BMI } \\
\hline Aktuelle Größe & \\
\hline \multirow{3}{*}{$\begin{array}{l}\text { Entscheidung zur } \\
\text { Adipositastherapie }\end{array}$} & - $\quad$ Art, auf die Familie von Sprechstunde erfahren hat \\
\hline & - Motivator der Therapie (Mutter/Vater/Kind/extern) \\
\hline & - Erwartungen an Sprechstunde \\
\hline \multirow{9}{*}{ Bewertung der Therapie im SPZ } & - Bewertung von \\
\hline & • „Anmeldung“ \\
\hline & • „Wartezeit“ \\
\hline & • „ärztlicher Betreuung“ \\
\hline & • „Ernährungsberatung“ \\
\hline & • „psychologischer Unterstützung“ \\
\hline & - $\quad$ skalarische Bewertung von Profit und Zufriedenheit \\
\hline & (von 1 (sehr gut) bis 5 (sehr schlecht)) \\
\hline & - $\quad$ Äußerung freier Kritik \\
\hline Therapieende & Grund für Beendigung der Therapie \\
\hline Änderungen des & Steigerung/Verringerung von \\
\hline \multirow[t]{3}{*}{ Freizeitverhaltens durch Therapie } & - Medienkonsum \\
\hline & - Körperlicher Aktivität \\
\hline & - $\quad$ Aktivität mit Freunden und Familie \\
\hline \multirow[t]{2}{*}{ Adipogene Verhaltensweisen } & $\begin{array}{l}\text { - regelmäßige sportliche Betätigung (zum Therapiezeitpunkt } \\
\text { bzw. aktuell) }\end{array}$ \\
\hline & - regelmäßiges Wiegen \\
\hline \multirow{3}{*}{$\begin{array}{l}\text { Lebenssituation und } \\
\text { sozioökonomische Daten }\end{array}$} & - Lebens-/ Wohnsituation \\
\hline & $\begin{array}{l}\text { - } \quad \text { Schul- und Berufsausbildung, aktuelle berufliche Situation } \\
\text { (ehemaliger Patient) }\end{array}$ \\
\hline & $\begin{array}{l}\text { - Schul- und Berufsausbildung, aktuelle berufliche Situation } \\
\text { (Eltern) }\end{array}$ \\
\hline
\end{tabular}

Für diese Parameter wurde als jeweils unabhängige Variable geprüft, ob sich ein Zusammenhang zu den BMI-SDS-Reduktionen der Patienten zu den drei Zeitpunkten $\mathrm{T}_{0}, \mathrm{~T}_{1}$ und $\mathrm{T}_{2}$ nachweisen ließ. Ziel dieser Maßnahme ist die Darstellung von Faktoren, die mit einer höheren (im Sinne von Positivprädiktoren) bzw. einer niedrigeren (im Sinne von Negativprädiktoren) BMI-SDS-Reduktion einhergehen. 


\subsection{Statistische Auswertung}

Die statistische Auswertung der vorliegenden Arbeit erfolgte mithilfe der Programme Microsoft Excel (Version 14.5.7) sowie den Statistikprogrammen Graphpad Prism (Version 6.0) und Statistica (Version 10).

Um statistisch signifikante Zusammenhänge zwischen den drei Gruppen der BMI-SDS-Reduktion über die verschiedenen Zeiträume und den zu prüfenden Parametern darzustellen, wurden je nach Art der Variablen verschiedene Tests durchgeführt, die in folgender Tabelle aufgeführt sind:

Tabelle 3: Durchgeführte statistische Tests auf signifikante Zusammenhänge zwischen den verschiedenen Parametern (als unabhängige Variable) und der BMI-SDS-Reduktion (als abhängige Variable) in Abhängigkeit von der Art des jeweiligen Datensatzes

\begin{tabular}{ll}
\hline Unabhängige Variable & Statistischer Test \\
\hline Metrisch skaliert & $\begin{array}{l}\text { Untersuchung auf Korrelation mittels Korrelationskoeffizient } \\
\text { Spearman's Rho }\end{array}$ \\
\hline Nominal skaliert & $-\quad$ dichotom: t-Test (ungepaart) \\
Ordinal skaliert & $-\quad$ nicht dichotom: einfaktorielle Varianzanalyse (ANOVA) \\
& $\begin{array}{l}\text { Untersuchung auf Korrelation mittels Korrelationskoeffizient } \\
\text { Kendall's Tau }\end{array}$ \\
\hline
\end{tabular}

Bei allen oben genannten Tests wurde auf signifikante Zusammenhänge mit den BMI-SDSReduktionen der drei Gruppen über die verschiedenen Zeiträume als abhängige Variablen untersucht. Ziel war die Ermittlung von Parametern, die mit einer langfristig erfolgreichen Gewichtsabnahme in Zusammenhang stehen.

Für statistisch signifikante Parameter sowie das Therapiekonzept erfolgte weiterhin eine Überprüfung auf signifikante Zusammenhänge untereinander (Tabelle 4). Dies diente der Aufdeckung eventueller, durch Vermittlung über einen weiteren Faktor lediglich vorgetäuschter Zusammenhänge zur BMI-SDS-Reduktion. Die Aufnahme des Therapiekonzepts trotz fehlendem signifikanten Einfluss auf die BMI-SDS-Reduktion begründet sich in der Bedeutsamkeit dieses Parameters hinsichtlich des Ziels der vorliegenden Arbeit: der Optimierung von Therapieangeboten durch Erfassung von Prädiktoren für eine erfolgreiche Adipositastherapie im Kindes- und Jugendalter.

Tabelle 4: Durchgeführte statistische Tests auf signifikante Zusammenhänge zwischen den verschiedenen Parametern (als unabhängige Variable) in Abhängigkeit von der Art des jeweiligen Datensatzes

\begin{tabular}{|c|c|c|c|}
\hline 1. Parameter & 2. Parameter & \multicolumn{2}{|l|}{ Statistischer Test } \\
\hline Metrisch skaliert & Metrisch skaliert & \multirow{4}{*}{\multicolumn{2}{|c|}{$\begin{array}{l}\text { Untersuchung auf Korrelation mittels Korrelationskoeffizient } \\
\text { Spearman's Rho }\end{array}$}} \\
\hline Metrisch skaliert & Ordinal skaliert & & \\
\hline Nominal skaliert & Ordinal skaliert & & \\
\hline Ordinal skaliert & Ordinal skaliert & & \\
\hline Metrisch skaliert & Nominal skaliert & $\begin{array}{l}\text { - nominaler Datensatz dichotom: } \\
\text { - nominaler Datensatz nicht dichotom: }\end{array}$ & $\begin{array}{l}\text { t-Test (ungepaart) } \\
\text { einfaktorielle Vari- } \\
\text { anzanalyse (ANOVA) }\end{array}$ \\
\hline Nominal skaliert & Nominal skaliert & $\begin{array}{l}\text { - } \quad \text { beide Datensätze dichotom: } \\
\text { - nicht beide Datensätze dichotom: }\end{array}$ & $\begin{array}{l}\text { Exakter Fisher-Test } \\
\text { Chi-Quadrat-Test }\end{array}$ \\
\hline
\end{tabular}


Für alle Tests wurde ein Signifikanzniveau von $\alpha=5$ Prozent festgelegt, wobei ein $p$-Wert $<$ 0,05 als signifikant betrachtet wurde. 


\section{Ergebnisse}

\subsection{Charakterisierung des Patientenkollektivs}

Im eingeschlossenen Zeitraum besuchten 442 Kinder und Jugendliche die AdipositasSprechstunde des SPZ (Abbildung 8). Von diesen wurden 358 Patienten in die Auswertung eingeschlossen. Davon waren 192 männlich (53,6 \%) und 166 weiblich (46,4 \%). Das durchschnittliche Alter bei Therapiebeginn lag bei 13,6 Jahren (KI: $\pm 3,5$ ). Das jüngste eingeschlossene Kind war 11,01 das älteste 17,95 Jahre alt. Der durchschnittliche BMI bei Therapiebeginn betrug 31,8 (KI: $\pm 11,6$ ) und lag im Durchschnitt auf dem 98,5. Perzentil (KI: \pm $6,0)$. Der durchschnittliche BMI-SDS lag bei 2,5 (KI: $\pm 1,1)$. Der größte Teil der Patienten war bei Therapiebeginn adipös $(43,8 \%)$ oder extrem adipös $(43,6 \%)$, darauf folgten übergewichtige Patienten (12,2\%).

Es konnten 128 Patienten durch Beantwortung des Fragebogens in die Follow-up-Erhebung aufgenommen werden. Von diesen gaben 117 aktuelle Gewichts- und Größendaten an. Für zusätzliche 32 Patienten konnten entsprechende Daten aus dem krankenhausinternen Patienteninformationssystem extrahiert werden. Damit konnten für insgesamt 149 der 358 Patienten (41,6 \%) Daten für den Follow-up-Zeitpunkt $\mathrm{T}_{2}$ erhoben werden, die Loss-to-Followup-Rate lag bei $58,4 \%$.

Von den 149 Patienten waren 87 männlich (58,4 \%) und 62 weiblich (41,6 \%). Das Durchschnittsalter zum Follow-up-Zeitpunkt lag bei 19,3 Jahren. Die durchschnittliche Therapiezeit lag bei 12,1 Monaten (KI: $\pm 23,4$ ), die durchschnittliche postinterventionelle Zeit bei 4,1 Jahren (KI: $\pm 4,8)$.

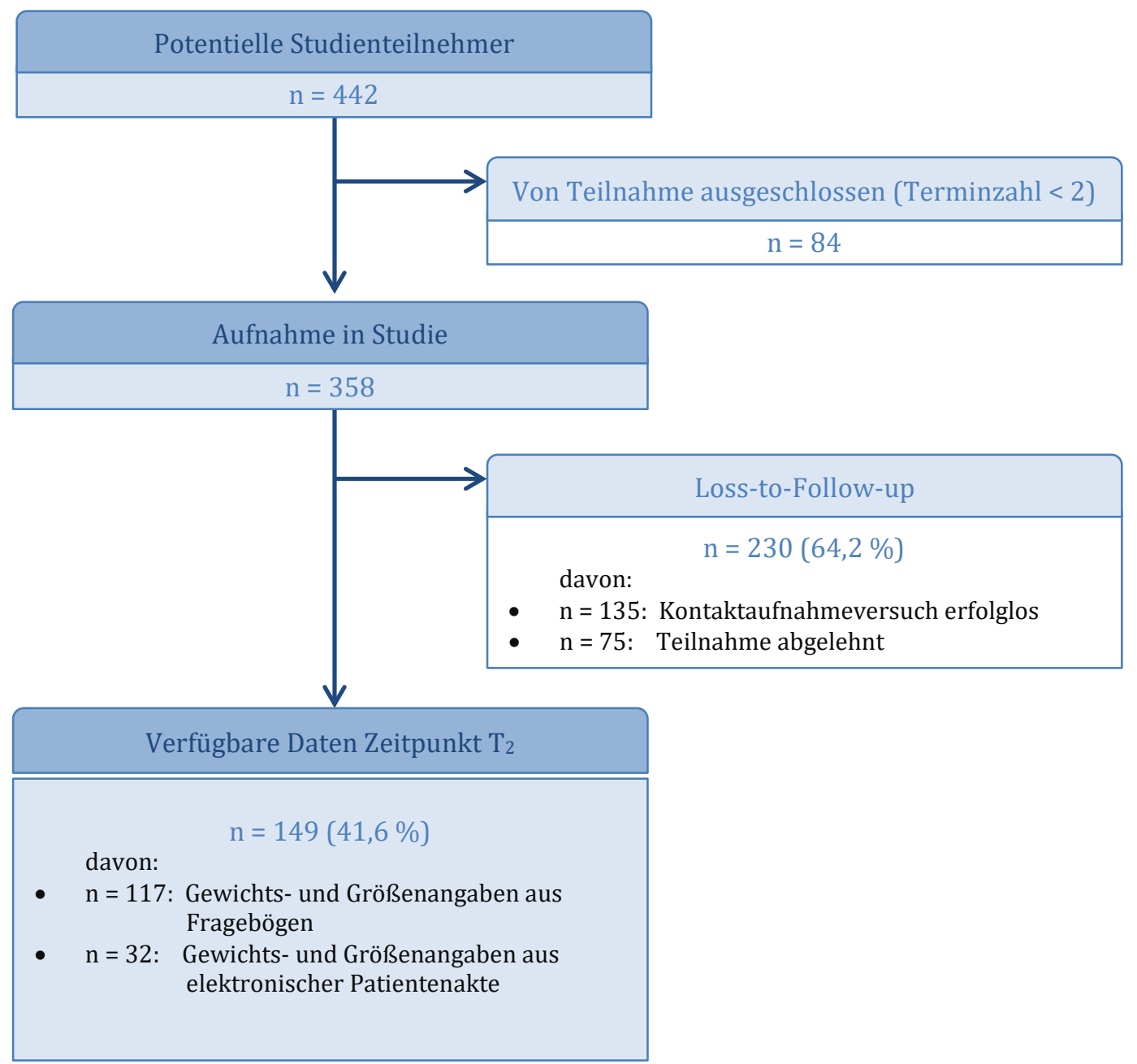


Abbildung 8: Flussdiagramm mit Darstellung der Patientenzahlen der in die Studie aufgenommenen Patienten, von der Teilnahme ausgeschlossenen Patienten sowie der Loss-to-Follow-up-Daten

\subsection{Therapieerfolg}

Bei der Erfassung des Therapieerfolgs wurde zum einen die Gewichtsreduktion über die verschiedenen Zeiträume beurteilt. Hier wurde zwischen einer kurzfristigen Entwicklung über den Zeitraum der Therapie (Differenz der Messwerte von Zeitpunkt $\mathrm{T}_{0}$ und $\mathrm{T}_{1}$ ) und einer langfristigen Entwicklung von Therapieende bis zum Follow-up-Zeitpunkt (Differenz der Messwerte zwischen $\mathrm{T}_{1}$ und $\mathrm{T}_{2}$ ) unterschieden. Für alle drei Zeiträume erfolgte jeweils eine Analyse nach pP- (ohne Einschluss des Loss-to-Follow-up; Tabelle 5) sowie nach ITT-Prinzip (unter Wertung eines Loss-to-Follow-up als Misserfolg mit Annahme einer BMI-SDS-Reduktion von 0; Tabelle 6). Abbildung 9 zeigt eine Auftragung der jeweiligen Durchschnittsreduktionen nach pP- bzw. ITT-Analyse gegeneinander.

Tabelle 5: Durchschnittlich erreichte kurzfristige, langfristige und gesamte BMI-SDS-Reduktion der eingeschlossenen Patienten nach pP-Analyse

\begin{tabular}{lcc}
\hline & BMI-SDS-Reduktion & Konfidenzintervall \\
\hline Kurzfristig $\left(\mathrm{T}_{0}-\mathrm{T}_{1}\right)$ & 0,07 & $\pm 0,08$ \\
Langfristig $\left(\mathrm{T}_{1}-\mathrm{T}_{2}\right)$ & 0,47 & $\pm 1,36$ \\
Gesamt $\left(\mathrm{T}_{0}-\mathrm{T}_{2}\right)$ & 0,56 & $\pm 1,55$ \\
\hline
\end{tabular}

Tabelle 6: Durchschnittlich erreichte kurzfristige, langfristige und gesamte BMI-SDS-Reduktion der eingeschlossenen Patienten nach ITT-Analyse

\begin{tabular}{lcc}
\hline & BMI-SDS-Reduktion & Konfidenzintervall \\
\hline Kurzfristig $\left(\mathrm{T}_{0}-\mathrm{T}_{1}\right)$ & 0,07 & $\pm 0,08$ \\
Langfristig $\left(\mathrm{T}_{1}-\mathrm{T}_{2}\right)$ & 0,17 & $\pm 0,97$ \\
Gesamt $\left(\mathrm{T}_{0}-\mathrm{T}_{2}\right)$ & 0,21 & $\pm 1,12$ \\
\hline
\end{tabular}

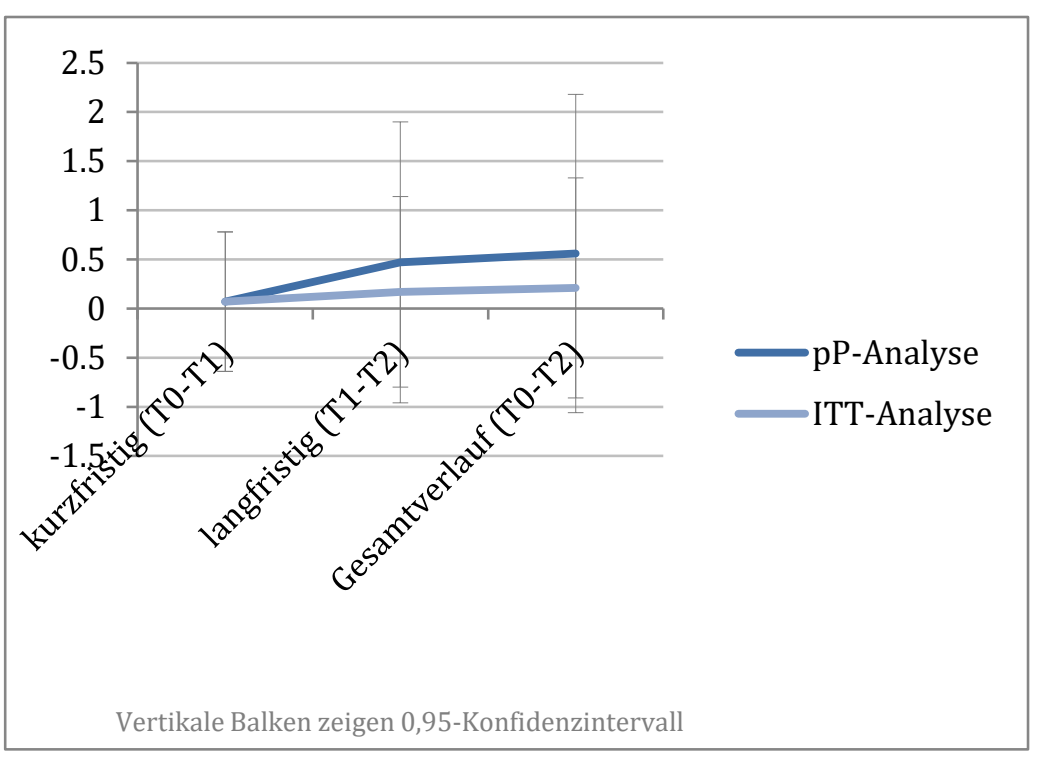

Abbildung 9: Gegenüberstellung der durchschnittlichen BMI-SDSReduktionen über Therapiezeit, postinterventionelle Zeit und Gesamtverlauf nach pP- und ITTAnalyse 
In Tabelle 7 sind die Prozentsätze der Patienten nach den Erfolgsdefinitionen der AGA aufgeführt. Danach entspricht eine BMI-SDS-Reduktion um mindestens 0,2 einem guten, um mindestens 0,5 einem sehr guten Therapieerfolg. Zusätzlich erfolgte eine Darstellung derjenigen Patienten, die überhaupt eine bzw. keinerlei Reduktion verzeichnen konnten. Die Analyse erfolgte nach pP- sowie ITT-Prinzip. In die ITT-Analyse gingen dabei Patienten, deren Daten zum Zeitpunkt $\mathrm{T}_{2}$ nicht erhoben werden konnten (entsprechend einem Loss-to-Follow-up), als nicht erfolgreich ein. Es wurde für diese Patienten eine BMI-SDS-Reduktion von 0 angenommen. Auffällig ist, dass der Prozentsatz an Patienten, die überhaupt eine Gewichtsreduktion erreichen konnten, nach pP-Analyse langfristig und gesamt deutlich ansteigt, während er sich nach ITTAnalyse verringert. Dagegen vergrößert sich der Anteil an Patienten mit guten bzw. sehr guten Erfolgen langfristig und über den Gesamtverlauf nach beiden Analyseformen im Vergleich zu den kurzfristigen Ergebnissen.

Tabelle 7: Kurzfristige, langfristige und gesamte Prozentsätze der Patienten ohne Gewichtsreduktion (BMI-SDSReduktion $\leq 0)$, mit Gewichtsreduktion (BMI-SDS-Reduktion >0), mit guter (BMI-SDS-Reduktion $\geq 0,2)$ sowie sehr guter (BMI-SDS-Reduktion $\geq 0,5$ ) nach pP- und ITT-Analyse

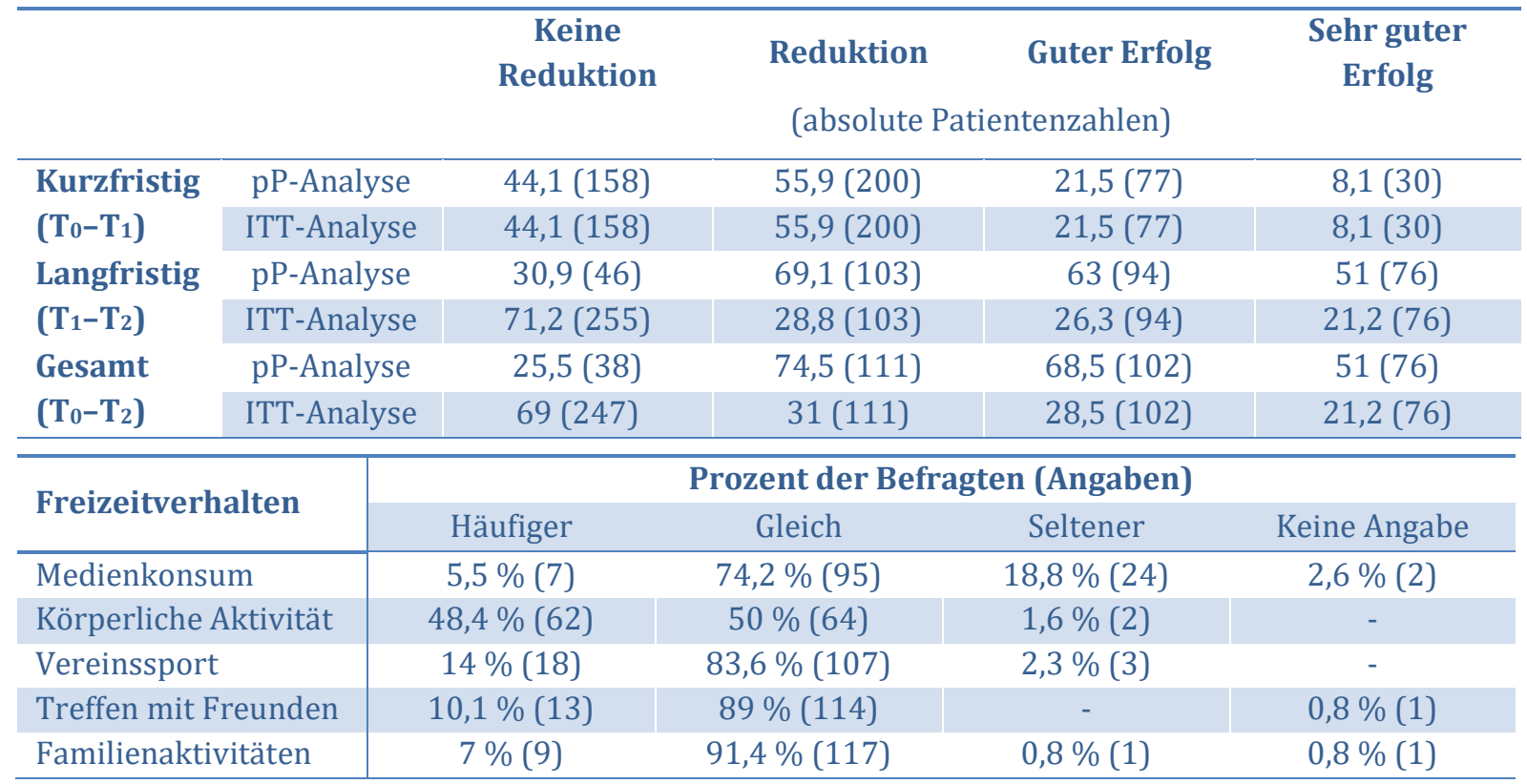

Zur weiteren Untersuchung des Therapieerfolgs wurde auch die Entwicklung des Bewegungsverhaltens erfasst. Hierzu wurde nach Veränderungen des Freizeitverhaltens im Rahmen der Therapie gefragt. Insgesamt konnte unter Betrachtung aller Parameter für bis zu $50,78 \%$ der Patienten eine positive Entwicklung (mit aktiverer Freizeitgestaltung) und für bis zu 8,59 \% eine negative Entwicklung des Freizeitverhaltens festgestellt werden. Eine detaillierte Darstellung findet sich in Tabelle 8.

\subsection{Untersuchung des Einflusses therapiebezogener Parameter auf die BMI-SDS-Reduktion}

\subsubsection{Therapie}

Tabellen 9 und 10 geben einen Überblick über die Behandlungen, die die eingeschlossenen Patienten erhalten haben. Bezüglich der Therapiekonzepte wurde in eine „multiprofessionelle Therapie“ mit Erhalt der drei Therapiebausteine „ärztliche Betreuung“, „Ernährungsberatung“ 
und „psychologische Betreuung“ und eine „individuelle Therapie“ mit Erhalt einer ärztlichen Betreuung und nur eines weiteren Bausteins („Ernährungsberatung“ oder „psychologische Betreuung") unterteilt. Der besseren Anschaulichkeit halber sind die Informationen aus Tabelle 10 in den Abbildungen 10 und 11 noch einmal grafisch dargestellt.

Tabelle 9: Durchschnittliche Dauer, postinterventionelle Zeit und Terminzahl der durchgeführten Therapie unter Angabe des Konfidenzintervalls.

\begin{tabular}{lcc}
\hline & Durchschnitt & Konfidenzintervall \\
\hline Therapiedauer & 12,1 Monate & $\pm 23,5$ \\
& (kürzeste: 2 Tage; längste: 4,8 Jahre) & \\
Postinterventionelle Zeit & 4,1 Jahre & \\
& (kürzeste: 1,0; längste 9,0 Jahre) & \\
Terminzahl/Therapie & 10,8 & $\pm 18,6$ \\
Ernährungsberatung & 4,3 & $\pm 9,0$ \\
Arzt & 3,4 & $\pm 5,2$ \\
Psychologie & 3,3 & $\pm 8,8$
\end{tabular}

Tabelle 10: Prozentsatz der jeweilig durchgeführten Therapie von allen Behandlungen der eingeschlossenen Patienten mit Angabe von Terminzahl und Therapiedauer sowie des entsprechenden Konfidenzintervalls (KI) (multiprofessionelle Therapie: Erhalt von ärztlicher Betreuung, Ernährungsberatung und psychologischer Betreuung; Einzeltherapie: Erhalt von ärztlicher Betreuung und Ernährungsberatung oder psychologischer Betreuung)

\begin{tabular}{|c|c|c|c|}
\hline Therapiekonzept & $\begin{array}{c}\text { Prozentsatz der } \\
\text { durchgeführten } \\
\text { Behandlungen }\end{array}$ & $\begin{array}{c}\text { Durchschnittlich } \\
\text { e Terminzahl } \\
\text { (KI) }\end{array}$ & $\begin{array}{c}\text { Therapiedauer } \\
\text { [Jahre] (KI) }\end{array}$ \\
\hline Multiprofessionelle Gruppentherapie & 8,7 & $23,5( \pm 30,1)$ & $1,5( \pm 2,4)$ \\
\hline Multiprofessionelle Einzeltherapie & 57,6 & $11,2( \pm 16,2)$ & $1,0( \pm 2,0)$ \\
\hline Individuelle Einzeltherapie, davon: & 27,9 & $5,5( \pm 7,2)$ & $0,7( \pm 1,8)$ \\
\hline Arzt und Ernährungsberatung & 79,0 & - & \\
\hline Arzt und Psychologie & 21,0 & - & \\
\hline Ausschließlich Arzttermine & 5,3 & $2,6( \pm 2,4)$ & $0,8( \pm 1,6)$ \\
\hline
\end{tabular}

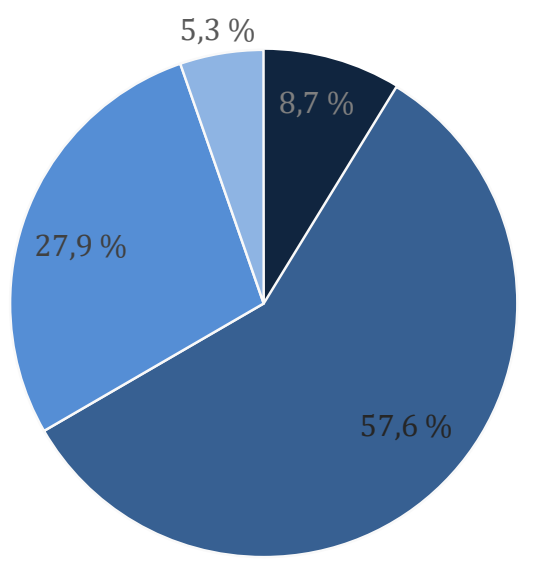

Abbildung 10: Prozentuale Verteilung der Patienten über die verschiedenen Therapiekonzepte 


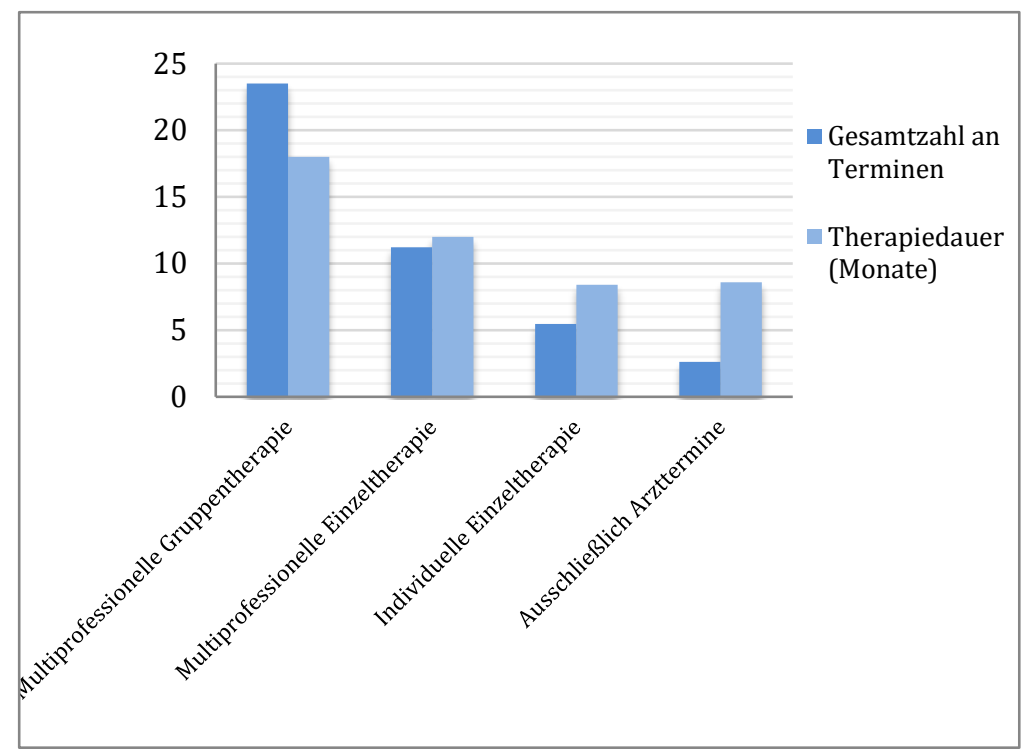

Abbildung 11: Durchschnittliche

Terminanzahl und Therapiedauer in Abhängigkeit vom Therapiekonzept

\subsubsection{Therapiekonzept}

Es konnten Unterschiede zwischen den durchschnittlichen BMI-SDS-Reduktionen in Abhängigkeit vom jeweiligen Therapiekonzept gesehen werden. Eine Darstellung der durchschnittlichen BMI-SDS-Reduktionen sowie der Erfolgsgruppen "Gewichtsreduktion", "guter Erfolg" (entspricht BMI-SDS-Reduktion von mindestens 0,2) und „sehr guter Erfolg“ (entspricht BMI-SDS-Reduktion von mindestens 0,5) der verschiedenen Therapiekonzepte findet sich in Abbildung 12 sowie Tabelle 11. Kurzfristig konnte die höchste durchschnittliche BMI-SDS-Reduktion von 0,10 (KI: $\pm 0,96$ ) von Patienten erreicht werden, die eine multiprofessionelle Gruppentherapie erhalten hatten. Langfristig fanden sich die höchsten Reduktionen von durchschnittlich 0,80 (KI: $\pm 0,66$ ) bei den 6 Patienten, die ausschließlich ärztliche Termine erhalten hatten. Über den Gesamtverlauf wurden die höchsten Reduktionen von durchschnittlich 0,71 (KI: $\pm 4,58$ ) ebenfalls von den Patienten mit multiprofessioneller

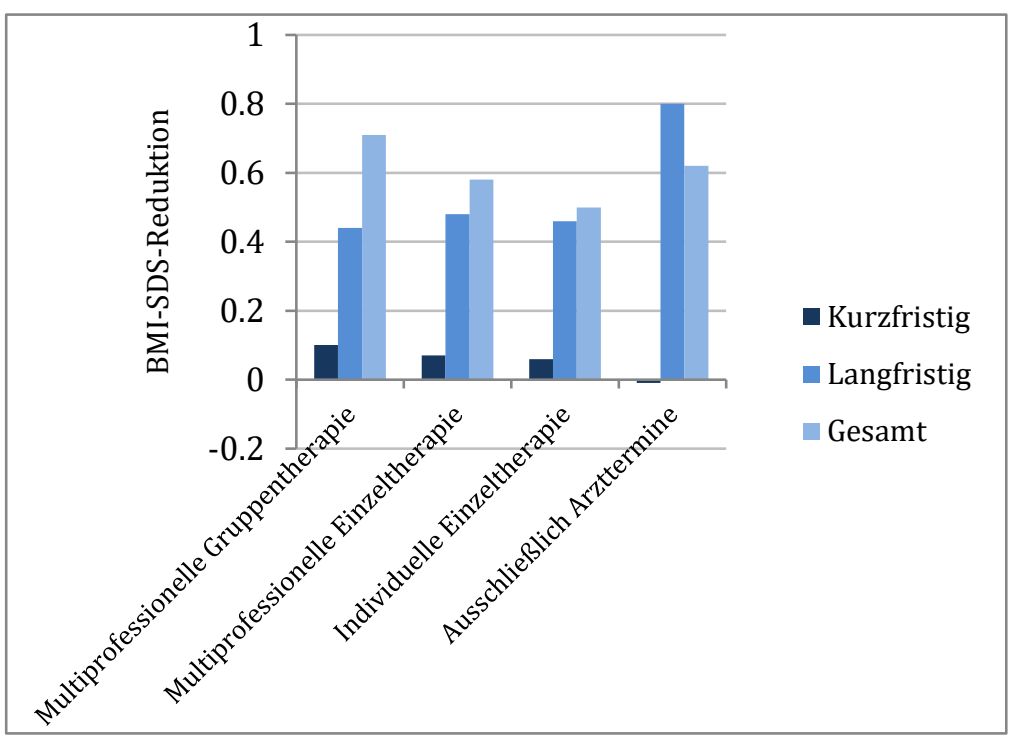

Gruppentherapie erzielt. Zwischen den Gruppen besteht bei p-Werten $>0,05$ jedoch kein signifikanter Unterschied.

Abbildung 12: Durchschnittliche kurzfristige, langfristige und gesamten BMI-SDS-Reduktion in Abhängigkeit vom Therapiekonzept 
Tabelle 11: Darstellung der kurzfristigen, langfristigen und gesamten durchschnittlichen BMI-SDS-Reduktion (unter Angabe des Konfidenzintervalls (KI)) bzw. der Erfolgsgruppen in Abhängigkeit vom jeweiligen Therapiekonzept

\begin{tabular}{lccccc}
\hline Therapie-konzept & $\begin{array}{c}\text { Patientenza } \\
\text { hl }\end{array}$ & $\begin{array}{c}\text { BMI-SDS- } \\
\text { Veränderung }\end{array}$ & $\begin{array}{c}\text { Gewichts- } \\
\text { reduktion }\end{array}$ & Guter Erfolg & $\begin{array}{c}\text { Sehr guter } \\
\text { Erfolg }\end{array}$ \\
\hline \multicolumn{7}{l}{ (KI) } & Prozentsatz (Absolute Zahl) \\
\hline \multicolumn{2}{l}{ Multiprofessionelle Gruppentherapie } \\
\hline Kurzfristig $\left(\mathrm{T}_{0}-\mathrm{T}_{1}\right)$ & 30 & $0,10( \pm 0,96)$ & $56,7(17)$ & $26,7(8)$ & $10,0(3)$ \\
Langfristig $\left(\mathrm{T}_{1}-\mathrm{T}_{2}\right)$ & 9 & $0,44( \pm 1,60)$ & $55,6(5)$ & $55,6(5)$ & $55,6(5)$ \\
Gesamt $\left(\mathrm{T}_{0}-\mathrm{T}_{2}\right)$ & 10 & $0,71( \pm 2,29)$ & $50,0(5)$ & $50,0(5)$ & $50,0(5)$
\end{tabular}

Multiprofessionelles Einzeltherapie

\begin{tabular}{|l|c|c|c|c|c|}
\hline Kurzfristig $\left(\mathrm{T}_{0}-\mathrm{T}_{1}\right)$ & 203 & $0,07( \pm 0,70)$ & $59,1(120)$ & $21,2(43)$ & $7,9(16)$ \\
\hline Langfristig $\left(\mathrm{T}_{1}-\mathrm{T}_{2}\right)$ & 81 & $0,48( \pm 1,52)$ & $70,4(57)$ & $64,2(52)$ & $50,6(41)$ \\
\hline Gesamt $\left(\mathrm{T}_{0}-\mathrm{T}_{2}\right)$ & 82 & $0,58( \pm 1,64)$ & $78,0(64)$ & $72,0(59)$ & $51,2(42)$ \\
\hline
\end{tabular}

Individuelle Einzeltherapie

\begin{tabular}{|l|l|l|l|c|c|}
\hline Kurzfristig $\left(\mathrm{T}_{0}-\mathrm{T}_{1}\right)$ & 97 & $0,06( \pm 0,84)$ & $55,7(54)$ & $21,6(21)$ & $9,3(9)$ \\
\hline Langfristig $\left(\mathrm{T}_{1}-\mathrm{T}_{2}\right)$ & 32 & $0,46( \pm 1,28)$ & $78,1(25)$ & $65,6(21)$ & $50,0(16)$ \\
\hline Gesamt $\left(\mathrm{T}_{0}-\mathrm{T}_{2}\right)$ & 32 & $0,50( \pm 1,36)$ & $75,0(24)$ & $71,8(23)$ & $56,3(18)$ \\
\hline
\end{tabular}

Ausschließlich Arzttermine

\begin{tabular}{l|c|c|c|c|c|}
\hline Kurzfristig $\left(\mathrm{T}_{0}-\mathrm{T}_{1}\right)$ & 17 & $-0,01( \pm 1,30)$ & $52,9(9)$ & $29,4(5)$ & $11,8(2)$ \\
\hline Langfristig $\left(\mathrm{T}_{1}-\mathrm{T}_{2}\right)$ & 6 & $0,80(0,66)$ & $100,0(6)$ & $100(6)$ & $100,0(6)$ \\
\hline Gesamt $\left(\mathrm{T}_{0}-\mathrm{T}_{2}\right)$ & 7 & $0,62(2,00)$ & $100,0(7)$ & $100(7)$ & $100,0(7)$ \\
\hline
\end{tabular}

Es erfolgte eine Untersuchung des Zusammenhangs zwischen Therapiekonzept und Parametern mit signifikanter Assoziation zur BMI-SDS-Reduktion über mindestens einen der drei Zeiträume (Tabelle 12). Dabei fiel ein signifikanter Zusammenhang zum Vorhandensein eines konkreten Zielgewichts vor Therapiebeginn auf: Patienten mit konkretem Zielgewicht erhielten signifikant intensivere Therapien (dabei wird von einer Zunahme der Therapieintensität in folgender Reihenfolge ausgegangen: ausschließlich ärztliche Termine - individuelle Einzeltherapie multiprofessionelle Einzeltherapie - multiprofessionelle Gruppentherapie). Gleiches galt für das Vorliegen einer Insulinresistenz.

Tabelle 12: Signifikante Zusammenhänge zwischen Therapiekonzept und weiteren Parametern mit signifikanter Assoziation zur BMI-SDS-Reduktion mit Angabe des $p$-Werts

\begin{tabular}{lc}
\hline Parameter & p \\
\hline Vorhandensein eines konkreten Zielgewichts & 0,001 \\
Vorliegen einer Insulinresistenz & 0,003 \\
\hline
\end{tabular}

Zu den Parametern „Steigerung der körperlichen Aktivität“, „Steigerung der sportlichen Aktivität im Verein“, „Profit von ärztlicher Betreuung“, „Zufriedenheit mit Ernährungsberatung“, „Profit von Ernährungsberatung“, „Alter bei Therapiebeginn“, „BMI-SDS bei Therapiebeginn“, „Geschlecht“, „Erhalt zusätzlicher kinder- und jugendpsychiatrischer Therapie“, „Abgeschlossene Berufsausbildung" sowie "Mitgliedschaft im Sportverein“ konnte kein signifikanter Zusammenhang festgestellt werden. 


\subsubsection{BMI-SDS-Reduktion über die Therapie}

Es wurden ebenfalls die Korrelation der BMI-SDS-Reduktionen über die verschiedenen Zeiträume untereinander untersucht. Dabei zeigte sich eine deutliche, hochsignifikante Korrelation $\left(\mathrm{r}^{2}=0,42 ; \mathrm{p}<0,0001\right)$ zwischen den Werten für die BMI-SDS-Reduktionen über den Therapiezeitraum ( $\mathrm{T}_{0}$ bis $\mathrm{T}_{1}$ ) und über den Gesamtzeitraum ( $\mathrm{T}_{0}$ bis $\mathrm{T}_{2}$ ) (Abbildung 13). Folglich sind höhere Gewichtsreduktionen im Rahmen der Therapie auch mit höheren Reduktionen über den Gesamtverlauf verknüpft. Zudem konnte eine ebenfalls deutliche und hochsignifikante Negativkorrelation $\left(\mathrm{r}^{2}=-0,35 ; \mathrm{p}<0,0001\right)$ zwischen den BMI-SDS-Werten zum Follow-up-Zeitpunkt $\mathrm{T}_{2}$ und der BMI-SDS-Reduktion über den Therapieverlauf festgestellt werden (Abbildung 14). Dementsprechend ist eine höhere Gewichtsreduktion im Rahmen der Therapie mit einem niedrigeren Gewicht zum Follow-up-Zeitpunkt assoziiert.

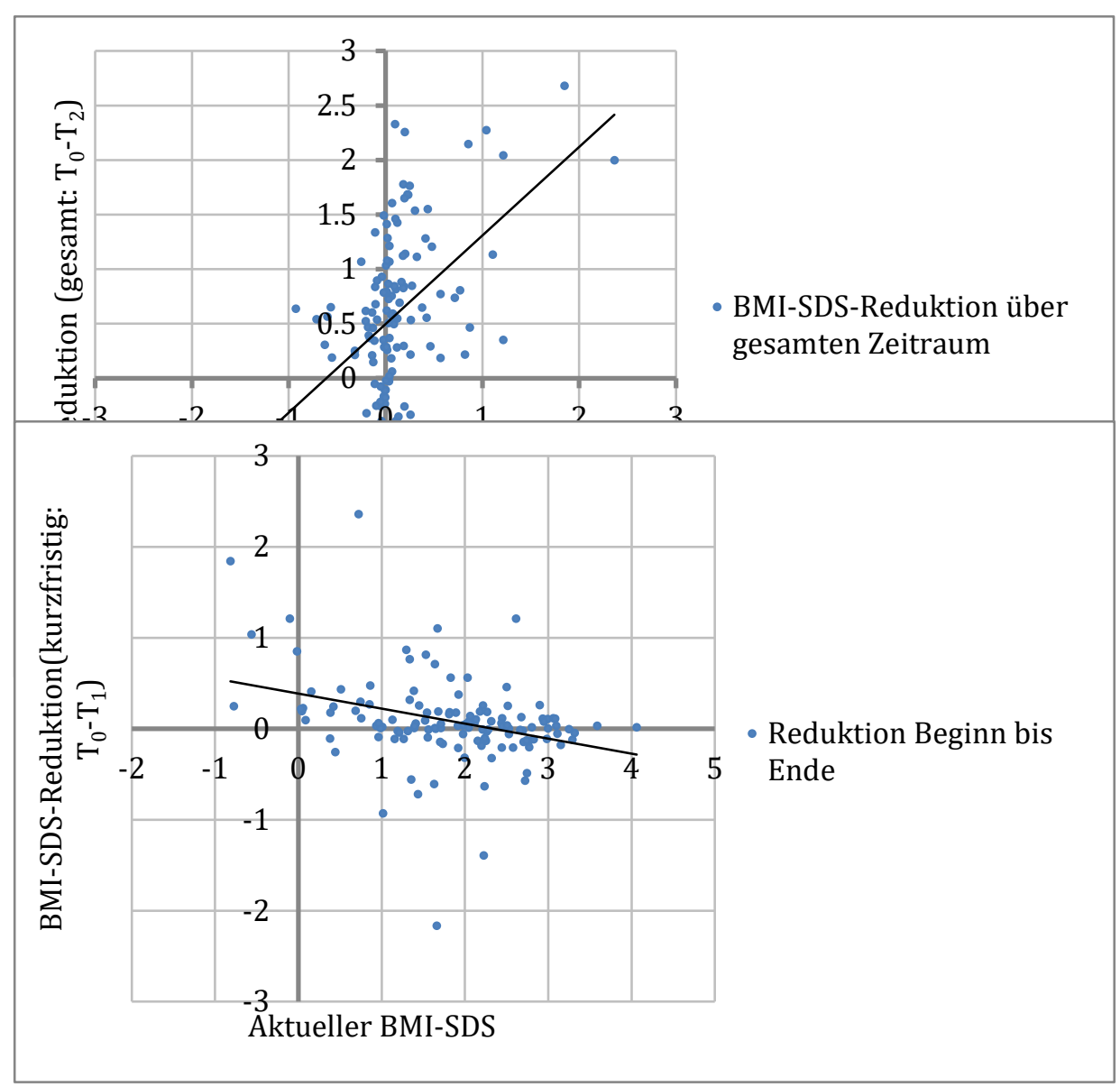

Abbildung 13: Lineare Regression mit Auftragung der BMISDS-Reduktion über den Therapieverlaufgegen die BMI-SDS-Reduktion über den gesamten Verlauf

Abbildung 14: Lineare Regression mit Auftragung des BMI-SDS zum Zeitpunkt $T_{2}$ gegen die BMI-SDS-Reduktion über den gesamten Therapiezeitraum

Auch zwischen den BMI-SDS-Reduktionen über den postinterventionellen Verlauf ( $T_{1}$ bis $\left.T_{2}\right)$ und den Gesamtverlauf ließ sich eine hochsignifikante Korrelation feststellen $\left(r^{2}=0,86, p<\right.$ 0,0001). Dagegen war kein Zusammenhang zwischen den Reduktionen über den Therapieverlauf und denen über die postinterventionelle Zeit darstellbar. 


\subsubsection{Entwicklung des Freizeitverhaltens}

Weiterhin konnten Zusammenhänge zwischen den BMI-SDS-Reduktionen über die verschiedenen Zeiträume sowie der Veränderung des Freizeitverhaltens im Rahmen der Therapie dargestellt werden (siehe Tabelle 8). Es zeigte sich ein signifikanter Zusammenhang ( $p$ $<0,05)$ zwischen der kurzfristigen BMI-SDS-Reduktion und einer Steigerung der körperlichen Aktivität im Allgemeinen $(\tau=0,18)$ sowie einer Steigerung der körperlichen Aktivität im Sportverein $(\tau=0,18)$ (Abbildung 15). Eine Steigerung der körperlichen Aktivität war folglich mit einer höheren Gewichtsreduktion assoziiert. Für die sportliche Betätigung im Verein konnten geringere langfristige $(\tau=0,05)$ und gesamte $(\tau=0,09)$ Korrelationen zur Gewichtsreduktion erfasst werden. Diese Ergebnisse liegen jedoch nicht im Rahmen des

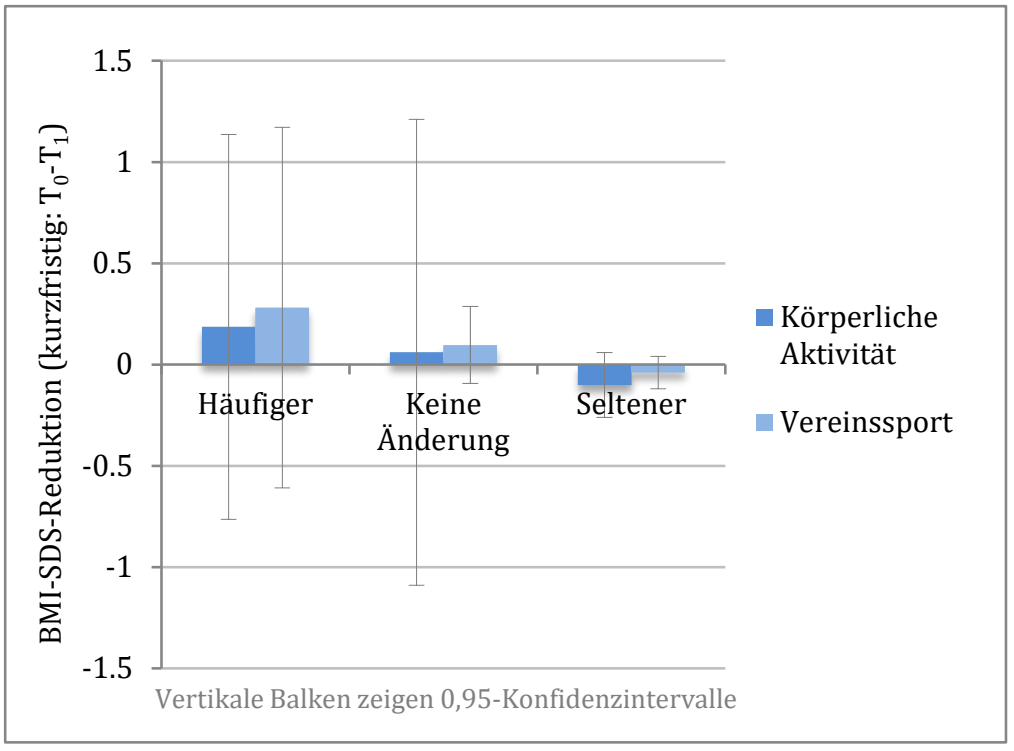

festgesetzten Signifikanzniveaus $(p>0,05)$. Für eine generelle Steigerung der körperlichen Aktivität war langfristig kein Zusammenhang mit einer höheren Gewichtsreduktion darstellbar. Für den Unterpunkt „Medienkonsum“ wurde kurzfristig $(\tau=0,08)$ und über den Gesamtverlauf $(\tau=0,04)$ eine Positivkorrelation erfasst. Diese Ergebnisse waren jedoch statistisch nicht signifikant $(\mathrm{p}>$ $0,05)$. Langfristig zeigte sich auch an dieser Stelle kein Zusammenhang. Für die Parameter „Aktivitäten mit Freunden" und „Familienaktivitäten“ waren ebenfalls keine signifikanten Zusammenhänge zur Gewichtsentwicklung nachweisbar.

Abbildung 15: Kurzfristige BMI-SDS-Reduktion in Abhängigkeit von der Entwicklung von körperlicher Aktivität und Ausübung eines Vereinssports im Rahmen der Therapie

Weiterhin wurden die Zusammenhänge zwischen der Steigerung der körperlichen Aktivität und anderen Parametern mit Assoziation zur BMI-SDS-Reduktion untersucht. Die Parameter, für die ein signifikanter Zusammenhang zur Aktivitätssteigerung erfasst werden konnte, sind in den Tabellen 13 bis 15 dargestellt. Patienten mit höherem Grad an körperlicher/sportlicher Aktivität nahmen insgesamt eine bessere Bewertung von Ernährungsberatung und Profit von der ärztlichen Betreuung vor. Ein höheres Alter bei Therapiebeginn sowie der Erhalt zusätzlicher psychiatrischer Interventionen (unabhängig von der psychiatrischen Betreuung im Rahmen der Adipositastherapie) waren dagegen mit einer geringeren Aktivitätssteigerung assoziiert. 
Für die Parameter „Zufriedenheit mit der ärztlichen Betreuung“, „BMI-SDS bei Therapiebeginn“ „Geschlecht", „Vorliegen einer Insulinresistenz" und „abgeschlossene Berufsausbildung“ konnte kein signifikanter Zusammenhang mit der Steigerung der körperlichen Aktivität festgestellt werden.

Es konnte weiterhin keine signifikante Assoziation zwischen der Steigerung der sportlichen Aktivität im Verein und den Parametern „Therapiekonzept“, „Profit von ärztlicher Betreuung“, "Zufriedenheit mit Ernährungsberatung“, „Vorhanden sein eines konkreten Zielgewichts vor Therapiebeginn“, „Alter bei Therapiebeginn“, „BMI-SDS bei Therapiebeginn“, „Geschlecht“, „Vorliegen einer Insulinresistenz“, „Erhalt zusätzlicher kinder- und jugendpsychiatrischer Therapie“ sowie „Abgeschlossene Berufsausbildung“ festgestellt werden.

Tabelle 13: Signifikante Zusammenhänge zwischen der Angabe zur Entwicklung der körperlichen Aktivität über die Therapie und weiteren ordinalen und metrischen Parametern mit signifikanter Assoziation zur BMI-SDS-Reduktion über mindestens einen der drei Zeiträume mit Angabe des jeweiligen Korrelationskoeffzienten $\left(r^{2}\right)$ sowie des $p$-Werts

\begin{tabular}{lcc}
\hline Parameter & $\mathrm{r}^{2}$ & $\mathrm{p}$ \\
\hline Steigerung Vereinssport & 0,45 & $9,25 \mathrm{e}^{-8}$ \\
\hline Profit von ärztlicher Betreuung & 0,30 & 0,0008 \\
Zufriedenheit mit Ernährungsberatung & 0,19 & 0,04 \\
\hline Profit von Ernährungsberatung & 0,33 & 0,0002 \\
\hline Alter bei Therapiebeginn & 0,3 & 0,0004 \\
\hline
\end{tabular}

Tabelle 14: Signifikante Zusammenhänge zwischen der Angabe zur Entwicklung der körperlichen Aktivität durch die Therapie und weiteren nominalen Parametern mit signifikanter Assoziation zur BMI-SDS-Reduktion über mindestens einen der drei Zeiträume. Dargestellt sind jeweils die durchschnittlichen Patientenangaben bei Vorliegen bzw. nichtVorliegen des Parameters und die entsprechenden $p$-Werte.

\begin{tabular}{l|c|c|c}
\hline Parameter & \multicolumn{2}{c}{$\begin{array}{c}\text { Durchschnittliche Angabe } \\
\text { bezüglich körperlicher Aktivität }\end{array}$} & p \\
& ja & nein & \\
\hline Zusätzliche psychiatrische Interventionen & "gleich“ & "häufiger“ & 0,04 \\
\hline Mitgliedschaft im Sportverein & „häufiger" & "gleich“ & 0,04 \\
\hline
\end{tabular}

Tabelle 15: Signifikante Zusammenhänge zwischen der Angabe zur Entwicklung der Aktivität im Sportverein durch die Therapie und weiteren ordinalen und metrischen Parametern mit signifikanter Assoziation zur BMI-SDS-Reduktion über mindestens einen der drei Zeiträume mit Angabe des jeweiligen Korrelationskoeffzienten $\left(r^{2}\right)$ sowie des $p$-Werts

\begin{tabular}{lcc}
\hline Parameter & $\mathbf{r}^{2}$ & $\mathbf{p}$ \\
\hline Steigerung körperlicher Aktivität & 0,45 & $9,25 \mathrm{e}^{-8}$ \\
Profit von Ernährungsberatung & 0,23 & 0,01 \\
\hline
\end{tabular}

\subsubsection{Therapiedauer}


Für die Dauer der Therapie konnte über den Therapiezeitraum eine mäßige Positivkorrelation der Werte mit der erreichten Gewichtsreduktion erfasst werden $\left(r^{2}=0,05 ; p=0,37\right)$. Langfristig ergaben sich ähnliche Ergebnisse $\left(r^{2}=0,04 ; p=0,6\right)$. Diese dargestellten Korrelationen sind bei recht hohen p-Werten wenig aussagekräftig. Über den Gesamtverlauf zeigte sich dagegen ein etwas deutlicherer Zusammenhang $\left(r^{2}=0,09 ; p=0,29\right.$, Abbildung 16). Damit besteht laut den Gesamtergebnissen eine Assoziation zwischen einer längeren Therapiedauer und einer größeren Gewichtsreduktion. Bei p-Werten $>0,05$ ist diese jedoch nicht statistisch gesichert.

Abbildung 16: Lineare Regression mit Auftragung der Therapiedauer gegen die BMI-SDS-Reduktion über den gesamten Verlauf

\subsubsection{Beendigung der Therapie}

Die Gründe für eine Beendigung der Therapie waren vielfältig (Tabelle 16). Der weitaus größte Teil der Patienten (53 Angaben unter Berücksichtigung von Mehrfachnennungen) gab an, dass

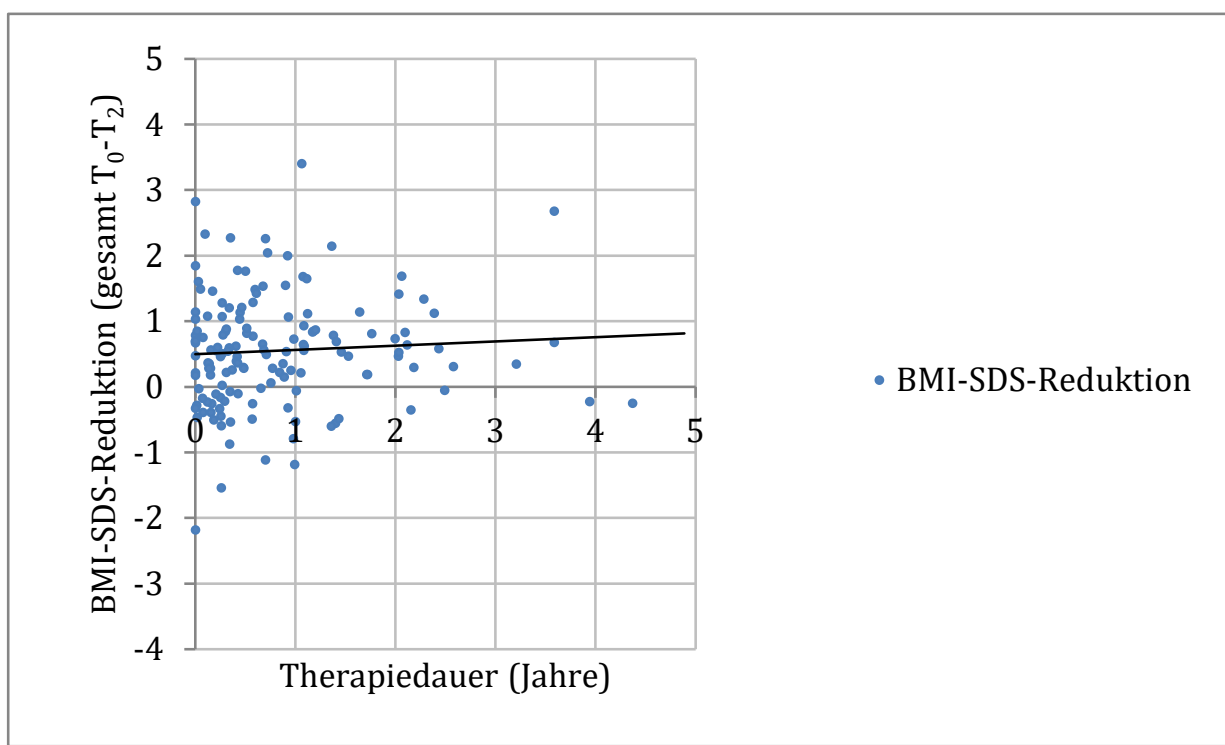

aufgrund eines Therapieerfolgs nicht fortgeführt zu haben. der Patient selbst keine Fortführung der Therapie wünschte. Damit spielte dieser Faktor bei $41,4 \%$ der beteiligten Familien eine Rolle für die Beendigung der Therapie. Auch mangelnder Erfolg hatte mit 17 Angaben bei 13,3\% der Familien einen Einfluss. Nur 11 der befragten Patienten (8,6 \%) gaben an, die Behandlung

Tabelle 16: Darstellung der Gründe für die jeweilige Therapie-Beendigung der zum Follow-up-Zeitpunkt befragten Patienten (Mehrfachnennungen möglich; Darstellung in absteigender Häufigkeit)

\begin{tabular}{lc}
\hline Grund für Beendigung der Therapie & Prozentzahlen (Zahl der Angaben) \\
\hline Fortführung von Patient nicht gewünscht & $41,4 \%(53)$ \\
\hline Mangelnder Erfolg & $13,3 \%(17)$ \\
Anfahrt zu weit & $11,7 \%(15)$
\end{tabular}




\begin{tabular}{l|c} 
Disharmonisches Verhältnis zu Therapeuten & $9,4 \%(12)$ \\
\hline Therapieerfolg & $8,6 \%(11)$ \\
\hline Zeitmangel der Eltern & $5,5 \%(7)$ \\
\hline Umsetzung zu schwierig & $4,7 \%(6)$ \\
\hline Zeitmangel des Kindes & $3,9 \%(5)$ \\
\hline Lange Wartezeiten & $0,8 \%(1)$ \\
Wiedervorstellungstermin vergessen & - \\
\hline Andere & $21,8 \%(28)$ \\
\hline
\end{tabular}

Es bestand für die BMI-SDS-Reduktionen über den Zeitraum der Therapie ein signifikanter Unterschied zwischen den Mittelwerten dieser verschiedenen Gruppen. Eine entsprechende Darstellung findet sich in Abbildung 17. Langfristig und über den gesamten Zeitraum ließ sich kein signifikanter Unterschied mehr nachweisen.

Die höchsten Reduktionen konnten auch bei denjenigen Patienten festgestellt werden, bei denen die Therapie aufgrund eines Therapieerfolgs beendet worden war. Patienten, bei denen die Therapie auf eigenen Wunsch oder aufgrund von Zeitmangel des Patienten selbst abgebrochen wurde, wiesen im Schnitt eine BMI-SDS-Zunahme auf. Gleiches gilt für diejenigen Patienten, die die Therapie aufgrund mangelnden Erfolges beendeten.

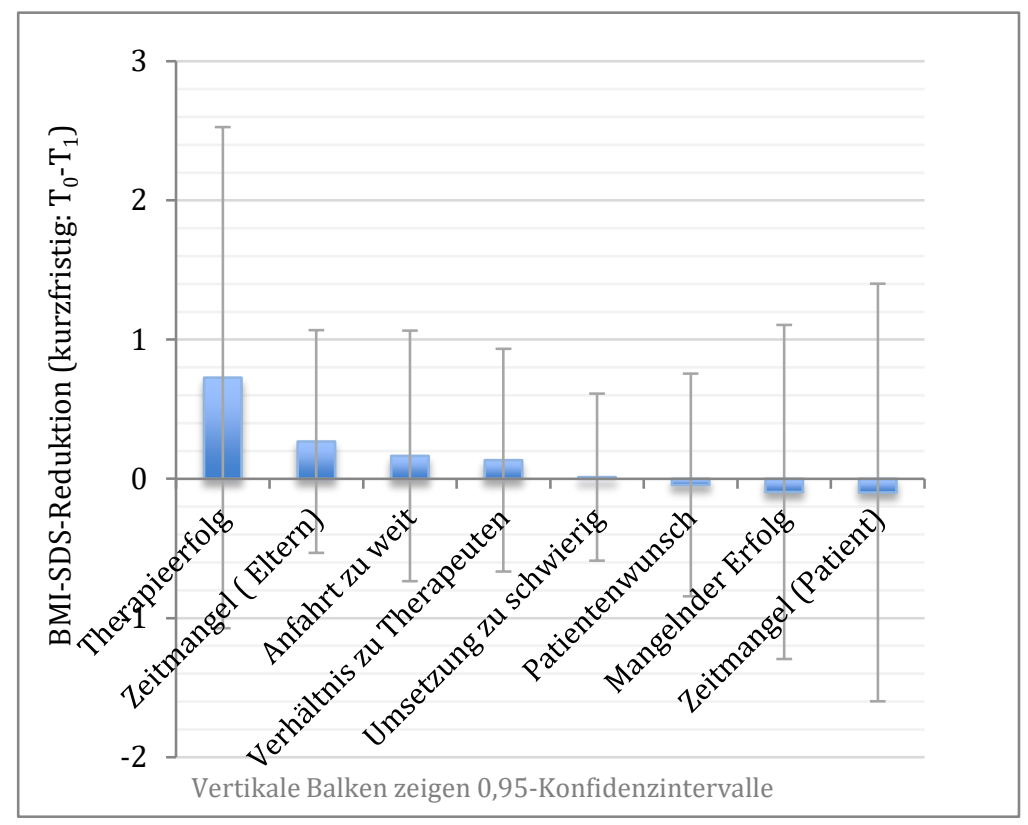

Abbildung 17: Durchschnittliche BMISDS-Reduktion über den

Therapiezeitraum in Abhängigkeit vom Grund für die Beendigung der Therapie

Ein Prozentsatz von 74,2 der Befragten (95 Patienten) gab an, dass er erneut ein Unterstützungsangebot des SPZ wahrnehmen würde. Etwa ein Viertel der Patienten (25,7 \%; 33 Patienten) lehnte dies ab.

\subsubsection{Bewertung der Therapie}

Zur Beurteilung der Arbeit des Teams für die Adipositasbehandlung im SPZ bzw. der einzelnen Therapeuten wurde eine skalarische Bewertung (1 (positiv) bis 5 (negativ)) vorgenommen (Tabelle 17). Unter Einschluss aller Faktoren wurde so für die Zufriedenheit mit der Therapie im SPZ ein Durchschnittswert von 2,4 (KI: $\pm 1,0$ ) erreicht, für den Profit von der Therapie ein Durchschnittswert von 3,39 (KI: $\pm 1,2$ ). Daneben bestand die Möglichkeit, freie Kritik zu äußern. Die verschiedenen positiven und negativen Bewertungen wurden verschiedenen Gruppen zugeordnet (Tabelle 18). 
Tabelle 17: Durchschnittliche Bewertung (von 1 (positiv) bis 5 (negativ)) der Abteilungen/ Therapeuten des SPZ zum Follow-up-Zeitpunkt mit Angabe von Zufriedenheit mit und Profit durch die einzelnen Instanzen unter Angabe des Konfidenzintervalls (KI)

\begin{tabular}{lcc}
\hline Bewertete Instanz & Zufriedenheit & Profit \\
\hline & \multicolumn{1}{c}{ Bewertung: 1 (KI) [Anzahl der Angaben] } \\
\hline Anmeldung & $1,98( \pm 1,80)[122]$ & - \\
Wartezeit & $2,42( \pm 2,02)[128]$ & - \\
Ärztliche Betreuung & $2,16( \pm 2,27)[128]$ & $3,17( \pm 2,78)[127]$ \\
Ernährungsberatung & $2,14( \pm 2,26)[125]$ & $2,88( \pm 2,79)[126]$ \\
Psychologische Betreuung & $2,44( \pm 2,53)[85]$ & $3,29( \pm 2,75)[84]$ \\
\hline
\end{tabular}

Tabelle 18: Positive und negative Kritik an ärztlicher Betreuung, Ernährungsberatung und psychologischer Betreuung der zum Follow-up-Zeitpunkt befragten Patienten

\begin{tabular}{lc}
\hline Kritik an ärztlicher Betreuung & \\
\hline Positiv & Angaben \\
\hline Medizinische Ab- und Aufklärung & 6 \\
\hline Freundlichkeit & 5 \\
\hline Individualität & 4 \\
\hline Motivation & 3 \\
\hline Sonstiges & 6
\end{tabular}

\begin{tabular}{lc}
\hline Negativ & Angaben \\
\hline Mangelnde Empathie/Freundlichkeit & 11 \\
\hline Mangelnde Einbeziehung des Kindes & 5 \\
\hline Übermäßiger Druck & 5 \\
\hline Seltenheit der Termine & 4 \\
\hline Mangel an Änderungsvorschlägen & 3 \\
\hline Mangelnde medizinische Abklärung & 3 \\
\hline Sonstiges & 12
\end{tabular}

\begin{tabular}{lc}
\hline \multicolumn{2}{l}{ Kritik an Ernährungsberatung } \\
\hline Positiv & Angaben \\
\hline Faustregeln für den Alltag & 19 \\
\hline Kindgerechtigkeit & 8 \\
\hline Rezepte & 7 \\
Praktische Anteile & 3 \\
Einbeziehung von Eltern und Kind & 3 \\
Sonstiges & 11
\end{tabular}

\begin{tabular}{lc}
\hline Negativ & Angaben \\
\hline Mangel an neuen Erkenntnissen & 13 \\
Mangel an praktischen Anteilen & 10 \\
\hline Unrealistische Zielsetzung & 7 \\
Therapie nicht intensiv genug & 3 \\
Sonstiges & 5
\end{tabular}

\begin{tabular}{lc}
\hline \multicolumn{2}{l}{ Kritik an psychologischer Betreuung } \\
\hline Positiv & Angaben \\
\hline Einbeziehung der Eltern & 2 \\
Freundlichkeit / Empathie & 2 \\
Kindgerechtigkeit & 2 \\
Sonstiges & 3
\end{tabular}

\begin{tabular}{lc}
\hline Negativ & Angaben \\
\hline Mangelnder Zugang zum Kind & 11 \\
\hline Therapie nicht intensiv genug & 8 \\
\hline Keine Hilfe für Gewichtsreduktion & 7 \\
Mangelnde Rücksicht auf Elternmeinung & 5 \\
Geringe inhaltliche Variabilität & 3 \\
\hline Sonstiges & 12
\end{tabular}

Es konnte ein signifikanter Zusammenhang zwischen dem angegebenen Profit von der ärztlichen Betreuung und der erreichten BMI-SDS-Reduktion über die Therapiezeit $(\tau=0,14)$ und den Gesamtverlauf ( $\tau=0,17$ ) festgestellt werden (Abbildung 18). Dabei war eine höhere Angabe für den Profit auch mit einer größeren Gewichtsabnahme assoziiert. Für die Zufriedenheit mit der ärztlichen Betreuung konnten dagegen keine signifikanten Zusammenhänge sowie nur geringere Korrelationen $(\tau=0,04)$ dargestellt werden.

In Hinblick auf die Ernährungsberatung zeigten sich sowohl für die Zufriedenheit mit der Behandlung als auch für den angegebenen Profit signifikante Korrelationen mit der Gewichtsreduktion. Für die Zufriedenheit konnten kurzfristig $(\tau=0,15)$ und über den gesamten Verlauf $(\tau=0,14)$ Zusammenhänge festgestellt werden $(p<0,05)$ (Abbildung 19). Langfristig 
zeigten sich etwas geringere Korrelationen $(\tau=0,09)$, die jedoch oberhalb des Signifikanzniveaus lagen $(p>0,05)$. Für die Angaben zum Profit von der Ernährungsberatung ergaben sich sogar noch deutlichere und ebenfalls signifikante Korrelationen für den Therapiezeitraum $(\tau=0,16)$ sowie über den Gesamtverlauf $(\tau=0,16 ; p<0,05)$ (Abbildung 20). Auch hier zeigte sich langfristig ein geringerer und nicht signifikanter Zusammenhang $(\tau=0,9$; $\mathrm{p}>0,05)$. Damit waren über den Therapiezeitraum und den Gesamtverlauf höhere Angaben für Zufriedenheit mit und Profit von der Ernährungsberatung mit einer größeren Gewichtsreduktion assoziiert.

Für die psychologische Unterstützung wurden sowohl für die Angaben zur Zufriedenheit als auch für die Angaben zum Profit vergleichsweise geringere Korrelationen festgestellt $(\tau=0,08$, jeweils höchster erreichter Wert, jeweils für den Gesamtverlauf). Diese lagen jedoch über dem festgelegten Signifikanzniveau.

Abbildung 18: Lineare Regression mit Auftragung der kurzfristigen (A) bzw. gesamten (B) BMI-SDS-Reduktion gegen die Angaben zum Profit mit der ärztlichen Betreuung

Abbildung 19: Lineare Regression mit Auftragung der kurzfristigen (A) bzw. gesamten (B) BMI-SDS-Reduktion gegen die
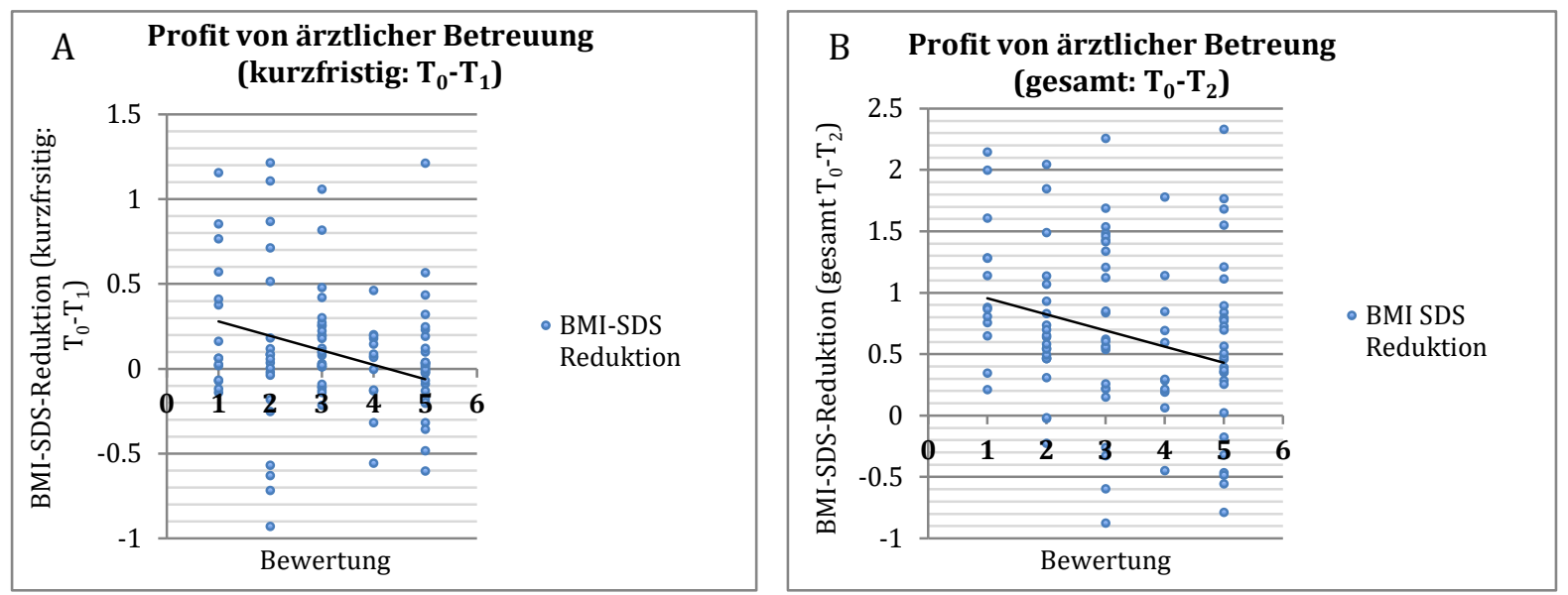

Angaben zur Zufriedenheit mit der Ernährungsberatung
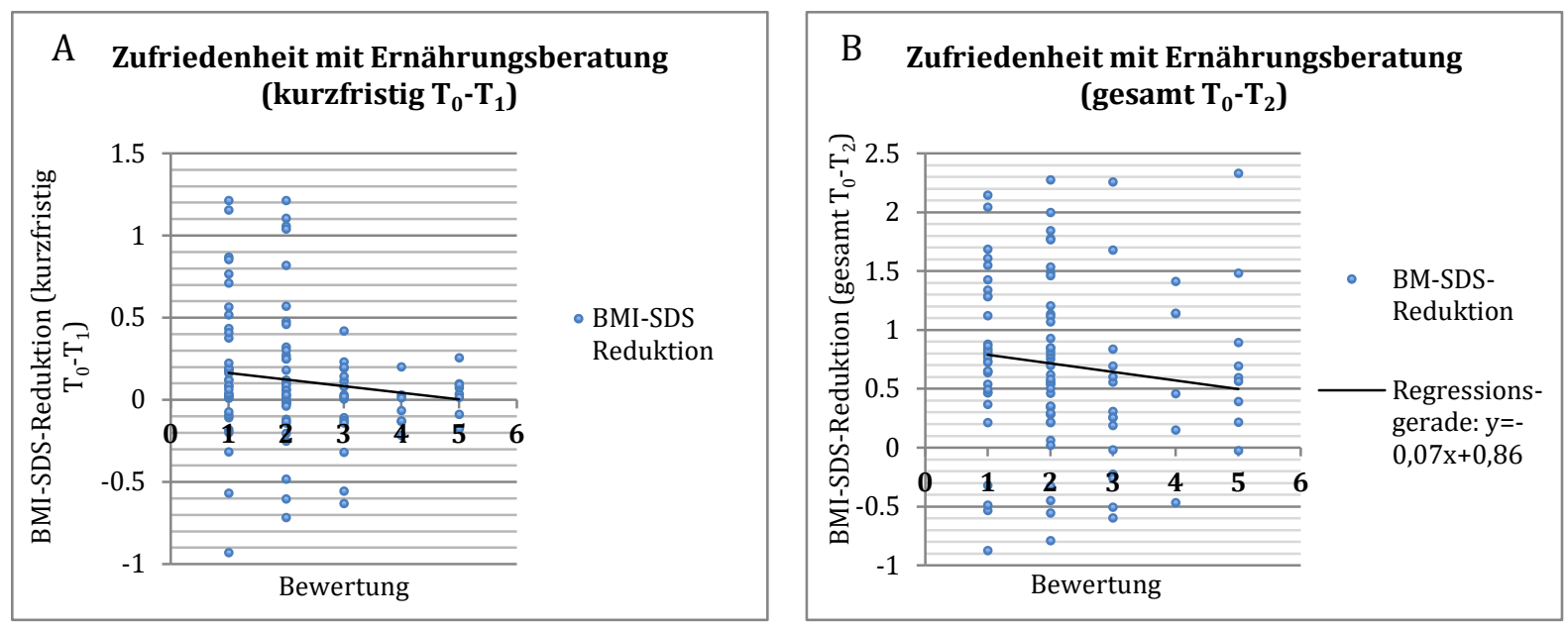

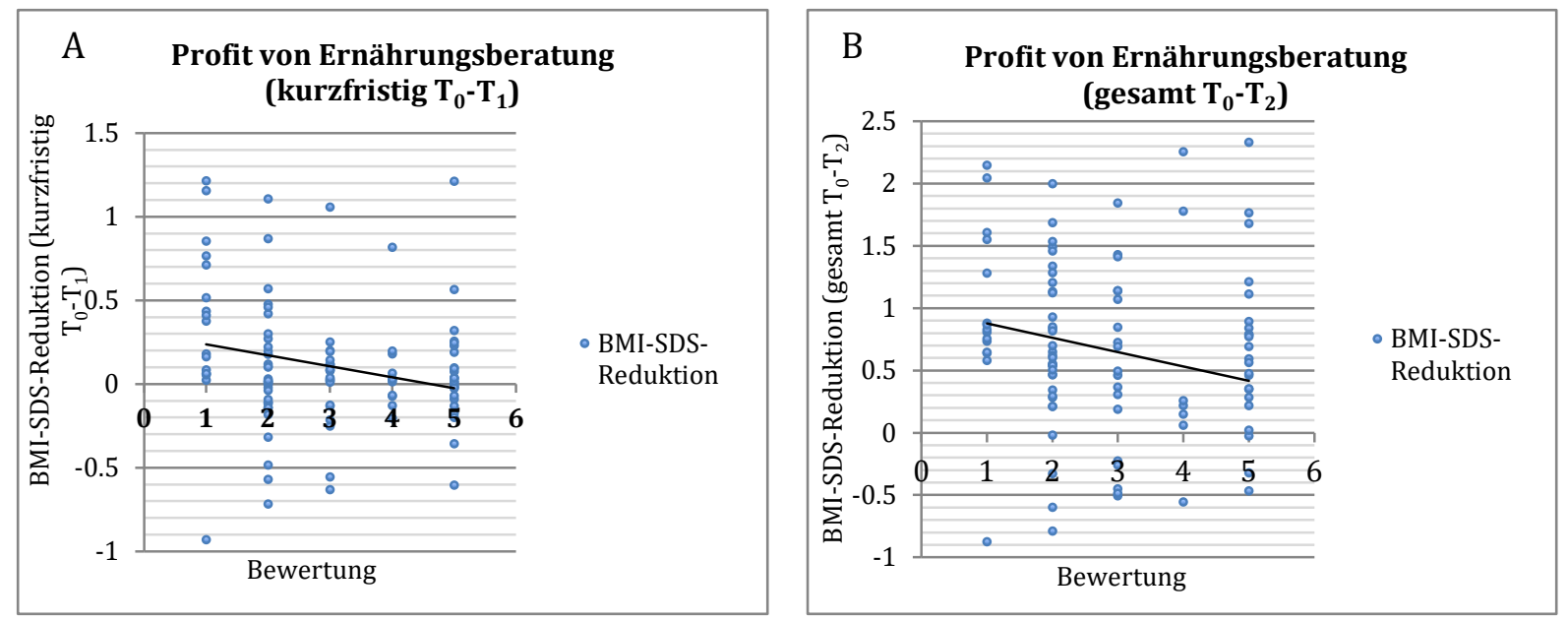

Abbildung 20: Lineare Regression mit Auftragung der kurzfristigen (A) bzw. gesamten (B) BMI-SDS-Reduktion gegen die Angaben zum Profit von der Ernährungsberatung

Zusätzlich erfolgte eine Untersuchung des Zusammenhangs zu anderen Parametern mit Assoziation zur BMI-SDS-Reduktion über mindestens einen der drei Zeiträume (Tabellen 19 22). Die Angaben zum „Profit durch die ärztliche Betreuung“, „Zufriedenheit mit der Ernährungsberatung" und „Profit durch die Ernährungsberatung" waren jeweils positiv miteinander assoziiert. Damit gingen positive Bewertungen der Ernährungsberatung auch mit positiven Bewertungen der ärztlichen Termine einher, eine höhere Angabe zur Zufriedenheit mit der Ernährungsberatung auch mit einem höheren Profit von dieser. Bessere Therapiebewertungen waren weiterhin mit einer stärkeren Steigerung der körperlichen Aktivität assoziiert.

Tabelle 19: Signifikante Zusammenhänge zwischen den Angaben zum Profit von der ärztlichen Betreuung und weiteren ordinalen Parametern mit signifikanter Assoziation zur BMI-SDS-Reduktion über mindestens einen der drei Zeiträume mit Angabe von Korrelationskoeffizient $\left(r^{2}\right)$ und $p$-Wert.

\begin{tabular}{lcc}
\hline Parameter & $\mathbf{r}^{2}$ & $\mathbf{p}$ \\
\hline Zufriedenheit mit Ernährungsberatung & 0,23 & 0,01 \\
\hline Profit von Ernährungsberatung & 0,66 & $0,06 \mathrm{e}^{-7}$ \\
\hline Steigerung körperlicher Aktivität & 0,30 & 0,0008 \\
\hline
\end{tabular}

Tabelle 20: Signifikante Zusammenhänge zwischen den Angaben zum Profit von der ärztlichen Betreuung und weiteren nominalen Parametern mit signifikanter Assoziation zur BMI-SDS-Reduktion über mindestens einen der drei Zeiträume mit Angabe von Korrelationskoeffizient ( $\left.r^{2}\right)$ und $p$-Wert.

\begin{tabular}{|c|c|c|c|}
\hline Parameter & $\begin{array}{r}\text { Durschni } \\
\left.\text { gut }^{\prime \prime}\right)\end{array}$ & $\begin{array}{l}\text { von („sehr } \\
\text { hlecht“) }\end{array}$ & $\mathbf{p}$ \\
\hline \multirow{2}{*}{ Vorhandensein eines konkreten Zielgewichts } & ja & nein & \\
\hline & 2 & 3 & 0,02 \\
\hline
\end{tabular}

Für den Profit von der ärztlichen Betreuung zeigte sich keine signifikante Assoziation zu den Parametern „Therapiekonzept", „Steigerung der Aktivität im Sportverein“, „Alter bei Therapiebeginn“, „BMI-SDS bei Therapiebeginn“, „Geschlecht", „Vorliegen einer Insulinresistenz", „Erhalt zusätzlicher psychiatrischer Interventionen“, „Abgeschlossene Berufsausbildung" und „Mitgliedschaft im Sportverein“. 
Tabelle 21: Signifikante Zusammenhänge zwischen den Angaben zur Zufriedenheit mit der Ernährungsberatung und weiteren Parametern mit signifikanter Assoziation zur BMI-SDS-Reduktion über mindestens einen der drei Zeiträume mit Angabe von Korrelationskoeffizient $\left(r^{2}\right)$ und $p$-Wert.

\begin{tabular}{lcc}
\hline Parameter & $\mathbf{r}^{2}$ & $\mathbf{p}$ \\
\hline Profit von ärztlicher Betreuung & 0,23 & 0,01 \\
Profit von Ernährungsberatung & 0,53 & $2,28 \mathrm{e}^{-10}$ \\
Steigerung körperlicher Aktivität & 0,19 & 0,04 \\
\hline
\end{tabular}

Für die Zufriedenheit mit der Ernährungsberatung zeigte sich kein signifikanter Zusammenhang zu den Parametern „Therapiekonzept", „Steigerung der Aktivität im Sportverein“, „Vorhandensein eines konkreten Zielgewichts vor Therapiebeginn“, „Alter bei Therapiebeginn“, „BMI-SDS bei Therapiebeginn“, „Geschlecht“, „Vorliegen einer Insulinresistenz", „Erhalt zusätzlicher psychiatrische Behandlung“, „Abgeschlossen Berufsausbildung“ und „Mitgliedschaft im Sportverein“.

Tabelle 22: Signifikante Zusammenhänge zwischen den Angaben zum Profit von der Ernährungsberatung und weiteren Parametern mit signifikanter Assoziation zur BMI-SDS-Reduktion über mindestens einen der drei Zeiträume mit Angabe von Korrelationskoeffizient $\left(r^{2}\right)$ und $p$-Wert.

\begin{tabular}{lcc}
\hline Parameter & $\mathbf{r}^{2}$ & $\mathbf{p}$ \\
\hline Profit von ärztlicher Betreuung & 0,66 & $0,06 \mathrm{e}^{-17}$ \\
Zufriedenheit mit Ernährungsberatung & 0,53 & $2,28 \mathrm{e}^{-10}$ \\
Steigerung körperlicher Aktivität & 0,33 & 0,0002 \\
\hline Steigerung des Vereinssports & 0,23 & 0,01 \\
\hline
\end{tabular}

Es zeigte sich für den Profit von der Ernährungsberatung kein Zusammenhang zu den Parametern „Therapiekonzept“, „Vorhandensein eines konkreten Zielgewichts vor Therapiebeginn“, „Alter bei Therapiebeginn“, „BMI-SDS bei Therapiebeginn“, „Geschlecht“, „Vorliegen einer Insulinresistenz", „Erhalt zusätzlicher psychiatrische Behandlung“, „Abgeschlossen Berufsausbildung“ und „Mitgliedschaft im Sportverein“.

\subsection{Untersuchung des Einflusses therapieunabhängiger Parameter auf die BMI-SDS-Reduktion}

\subsubsection{Motivation vor Therapiebeginn und Therapieziel}

Der Großteil der befragten Patienten hatte vom Kinderarzt oder Hausarzt bzw. aus der Kinderklinik von der Adipositas-Sprechstunde im SPZ erfahren. Die Motivation, eine entsprechende Therapie zu beginnen, ging dabei in der Regel von der Mutter des Patienten aus. Unter Einbezug von Mehrfachnennungen gaben 89 der 128 befragten Patienten die Mutter als Motivator an. Nur in 38 Fällen wurde der Patient selbst genannt, danach folgte der Vater des Patienten (28 Angaben) und schließlich externe Instanzen wie Kinderarzt/Hausarzt oder die Kinderklinik (16 Angaben).

Von der Therapie erhoffte sich die Mehrzahl der Patienten (ebenfalls unter Berücksichtigung von Mehrfachnennungen) eine Ernährungsschulung (45 Angaben), aber häufig auch die Abklärung von Ursachen und Folgeerkrankungen (34 Angaben) und eine psychologische Unterstützung (9 Angaben). Bezüglich des Gewichts strebte eine Majorität von 91,4 \% (117 Angaben) der Patienten einen Gewichtsverlust an. Von diesen 117 Patienten hatten 12 (10,2 \%) eine konkrete Vorstellung bezüglich ihres Zielgewichts. Die Vorstellungen reichten von BMIReduktionen von 1,9 bis zu Reduktionen $15,6 \mathrm{~kg} / \mathrm{m}^{2}$, im Schnitt wurde eine BMI-Reduktion von $6,7 \mathrm{~kg} / \mathrm{m}^{2}$ (KI: $\pm 8,5 \mathrm{~kg} / \mathrm{m}^{2}$ ) angestrebt. Das Zielgewicht lag in jedem einzelnen Fall mindestens eine Kategorie (extreme Adipositas - Adipositas - Übergewicht - Normalgewicht) unter dem 
Ausgangsgewicht. In drei Fällen war sogar eine Reduktion um zwei Kategorien beabsichtigt, außerdem lag in 3 Fällen der angestrebte BMI unterhalb des 50. Perzentils.

Eine detaillierte Zusammenfassung der Ergebnisse hinsichtlich Motivation und Therapieziel findet sich in Tabelle 23.

Tabelle 23: Befragungsergebnisse bezüglich der Entscheidung zum Therapiebeginn

\begin{tabular}{|c|c|}
\hline Kenntnis von Sprechstunde durch & Prozent der Befragten (Zahl der Angaben) \\
\hline Kinderarzt/Hausarzt & $57 \%(73)$ \\
\hline Kinderklinik & $15,6 \%(20)$ \\
\hline Freunde/Verwandte & $7 \%(9)$ \\
\hline Medien (Internet etc.) & $5,5 \%(7)$ \\
\hline Andere & $11,7 \%(15)$ \\
\hline Keine Angabe & $3,1 \%(4)$ \\
\hline \multicolumn{2}{|l|}{ Motivation durch } \\
\hline Ausschließlich Mutter & $42,2 \%(54)$ \\
\hline Ausschließlich Patient & $14 \%(18)$ \\
\hline Ausschließlich Vater & $5,5 \%(7)$ \\
\hline Ausschließlich extern: & $10,2 \%(13)$ \\
\hline Kinderklinik & $5,5 \%(7)$ \\
\hline Hausarzt/Kinderarzt & $4,7 \%(6)$ \\
\hline Mutter und Vater & $10,2 \%(13)$ \\
\hline Mutter und Patient & $10,2 \%(13)$ \\
\hline Eltern und Patient & $4,7 \%(6)$ \\
\hline Mutter und Hausarzt/Kinderarzt & $1,8 \%(2)$ \\
\hline Vater und Hausarzt/Kinderarzt & $0,8 \%(1)$ \\
\hline Eltern und Hausarzt/Kinderarzt & $0,8 \%(1)$ \\
\hline \multicolumn{2}{|l|}{ Vorstellung von Sprechstunde } \\
\hline Keine konkrete Vorstellung & $57,8 \%(74)$ \\
\hline Konkrete Vorstellung (Mehrfachnennungen): & $42,2 \%(54)$ \\
\hline Ernährungsschulung & $35,1 \%(45)$ \\
\hline $\begin{array}{l}\text { Abklärung medizinischer Grund- und } \\
\text { Folgeerkrankungen }\end{array}$ & $18 \%(23)$ \\
\hline Abklärung anderer Ursachen & $8,6 \%(11)$ \\
\hline Psychologische Unterstützung & $7 \%(9)$ \\
\hline Gruppentherapie & $2,3 \%(3)$ \\
\hline Einzeltherapie & $1,8 \%(2)$ \\
\hline Andere & $3,9 \%(5)$ \\
\hline
\end{tabular}

Angestrebte Gewichtsentwicklung

\begin{tabular}{lc} 
Gewichtsreduktion: & $91,4 \%(117)$ \\
\hline Keine konkrete Vorstellung von Zielgewicht & $82,0 \%(105)$ \\
\hline Konkrete Vorstellung von Zielgewicht & $9,4 \%(12)$ \\
Gewicht halten & $2,4 \%(3)$ \\
Kein bestimmtes Ziel & $3,9 \%(5)$ \\
Keine Angabe & $2,3 \%(3)$ \\
\hline
\end{tabular}

Es ließ sich kein signifikanter Zusammenhang zwischen der Art, auf die die Patienten von der Sprechstunde erfahren hatten, und der Gewichtsreduktion feststellen. Ebenso ließ sich kein Unterschied für die Gewichtsreduktion in Abhängigkeit von der Person oder Instanz (Mutter, 
Vater, Patient oder extern), von der die Therapie motiviert worden war, feststellen. Dagegen zeigte sich in Bezug auf das Zielgewicht ein Trend in der Gewichtsreduktion. Im Schnitt konnten die 91,4 \% der Patienten, die eine Gewichtsreduktion anstrebten, eine höhere BMI-SDSReduktion erreichen, als die 2,4 \%, die lediglich auf eine Gewichtsstabilisierung abzielten (Abbildung 21).

Dabei war die BMI-SDS-Reduktion für letztere über den Therapiezeitraum durchschnittlich um 0,21 (KI: $\pm 0,70 ; p=0,56$ ) niedriger, langfristig wurde eine um 0,75 (KI: $\pm 0,90 ; p=0,26$ ) niedrigere Reduktion erreicht. Über den Gesamtverlauf war die durchschnittliche BMI-SDS-

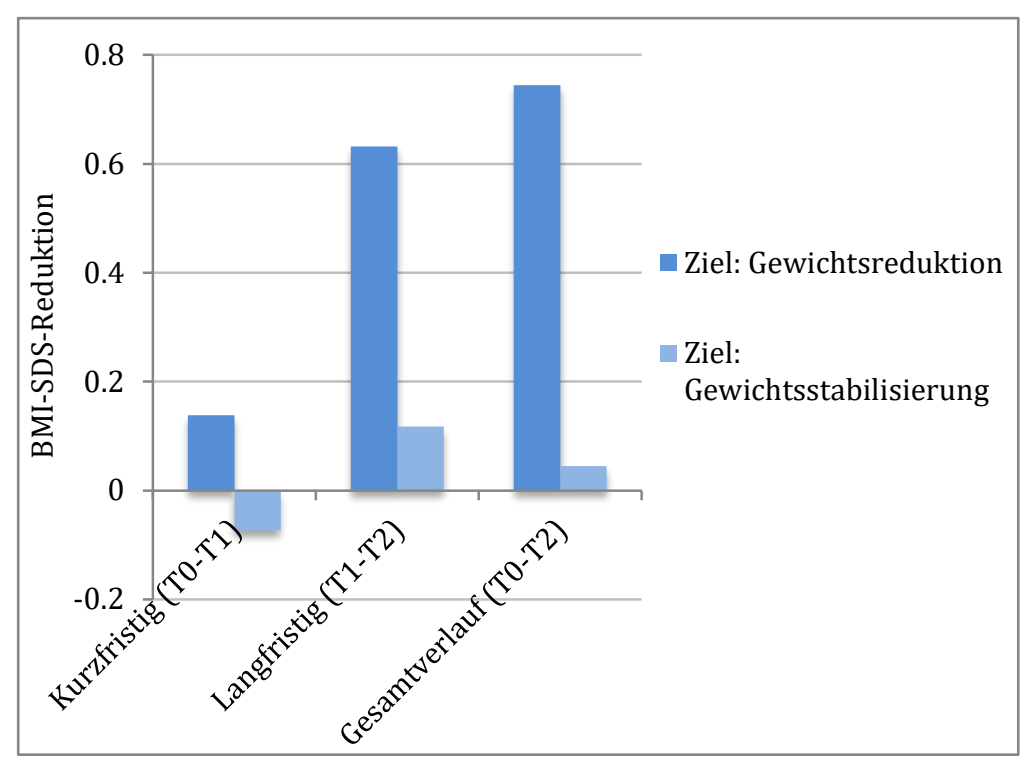

Reduktion der Patienten mit dem Ziel einer Gewichtsstabilisierung im Vergleich um 0,79 SDS-Punkte geringer (KI: $\pm 1,02 ; p=0,17)$. Diese Ergebnisse waren allerdings bei einem p-Wert > 0,05 nicht signifikant.

Abbildung 21: Vergleich der kurzfristigen, langfristigen und gesamten BMI-SDS-Reduktion zwischen Patienten mit dem Ziel einer Gewichtsreduktion bzw. -stabilisierung

Weiterhin bestand ein signifikanter Unterschied zwischen der Reduktion der zwölf Patienten mit konkreten Vorstellungen bezüglich ihres Zielgewichts im Gegensatz zu den Patienten ohne konkrete Vorstellungen (Abbildung 22). Langfristig lag dabei die durchschnittlich erreichte Reduktion um 0,82 (KI: $\pm 0,46 ; p=0,0005$ ), insgesamt um 0,71 (KI: $\pm 0,52 ; p=0,007$ ) über der von Patienten ohne konkretes Gewichtsziel. Kurzfristig ließ sich dieser Zusammenhang mit einer BMI-SDS-Differenz von 0,08 (KI: \pm 0,34) ebenfalls darstellen, dieses Ergebnis lag jedoch über dem Signifikanzniveau ( $p$

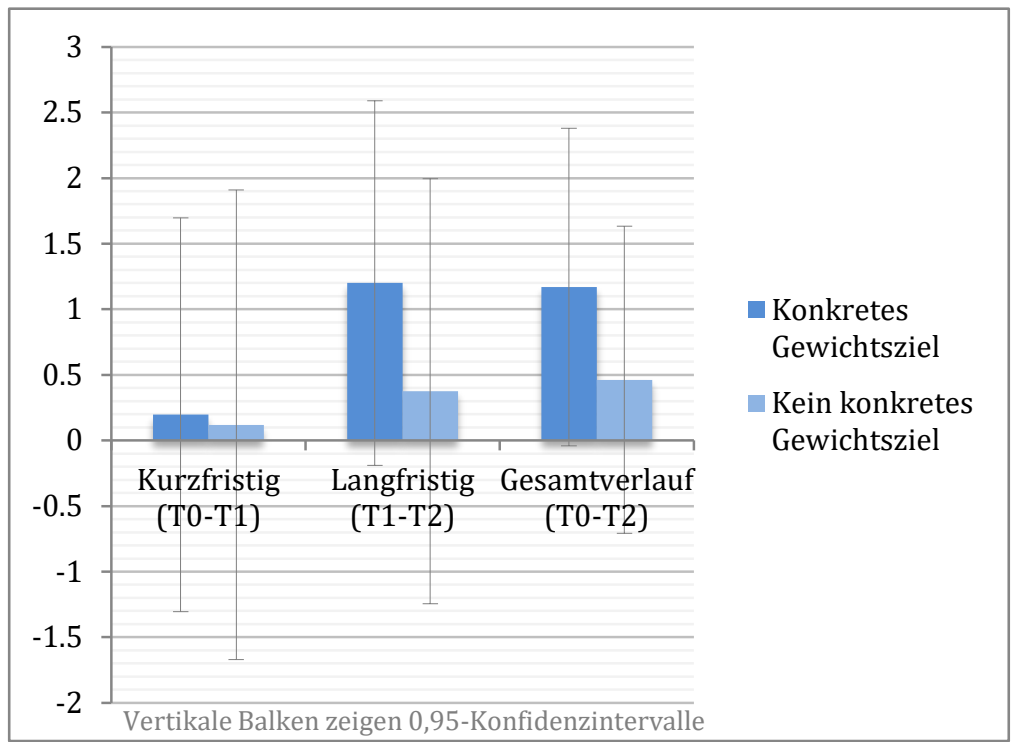
$=0,66)$.

Abbildung 22: Durchschnittliche kurzfristige, langfristige und gesamte BMI-SDS-Reduktion der Patienten mit bzw. ohne konkrete Vorstellungen bezüglich des angestrebten Gewichtsverlusts vor Therapiebeginn 
Bei der Untersuchung des Zusammenhangs zu weiteren für die BMI-SDS-Reduktion relevanten Parametern fiel ein Zusammenhang zu den Faktoren „Therapiekonzept" und "Profit von ärztlicher Betreuung" auf (Tabellen 24 und 25). Dabei war das Vorhandensein eines konkreten Zielgewichts vor Therapiebeginn mit einer signifikanten Zunahme der an der Therapie beteiligten Professionen assoziiert. Weiterhin war das Vorhandensein eines solchen Ziels mit einer signifikant besseren Bewertung des Profits von der ärztlichen Betreuung verknüpft.

Tabelle 24: Signifikanter Zusammenhang zwischen dem Vorhandensein eines konkreten Zielgewichts vor Therapiebeginn und dem nominalen Parameter „Therapiekonzept"

\begin{tabular}{lc}
\hline Parameter & $\mathbf{p}$ \\
\hline Therapiekonzept & 0,001 \\
\hline
\end{tabular}

Tabelle 25: Signifikanter Zusammenhang zwischen dem Vorhandensein eines konkreten Zielgewichts vor Therapiebeginn und dem ordinalen Parameter "Profit von ärztlicher Bewertung". Angegeben ist die jeweilige Durchschnittsbewertung der Therapie (von 1 („sehr gut) bis 5 (,sehr schlecht“)).

\begin{tabular}{l|c|c|c}
\hline Parameter & Durchschnittsbewertung & p \\
\hline Vorhandensein eines konkreten Zielgewichts & ja & nein & 0,02 \\
\hline
\end{tabular}

Es konnte kein signifikanter Zusammenhang zwischen dem Vorhandensein eines solchen Zielgewichts und den Parametern „Steigerung der körperlichen Aktivität“, „Steigerung der Aktivität im Sportverein“, „Zufriedenheit mit Ernährungsberatung“, „Profit durch Ernährungsberatung“, „Alter bei Therapiebeginn“, „BMI-SDS bei Therapiebeginn“, „Geschlecht“, „Vorliegen einer Insulinresistenz", „Erhalt zusätzlicher psychiatrische Behandlung“, „Abgeschlossen Berufsausbildung“ und „Mitgliedschaft im Sportverein“ erfasst werden.

\subsubsection{Alter bei Therapiebeginn}

Auch für das Alter der Patienten konnten signifikante Zusammenhänge mit dem Therapieerfolg festgestellt werden. So wurde eine signifikante Negativkorrelation zwischen dem Alter bei Therapiebeginn und der kurzfristigen BMI-SDS-Reduktion erfasst $\left(r^{2}=-0,11 ; p=0,04\right.$; Abbildung 23). Damit war ein höheres Alter mit einer geringeren Gewichtsreduktion assoziiert. 
Abbildung 23: Lineare Regression mit Auftragung der kurzfristigen BMI-SDS-Reduktion gegen das Alter bei Beginn der Therapie

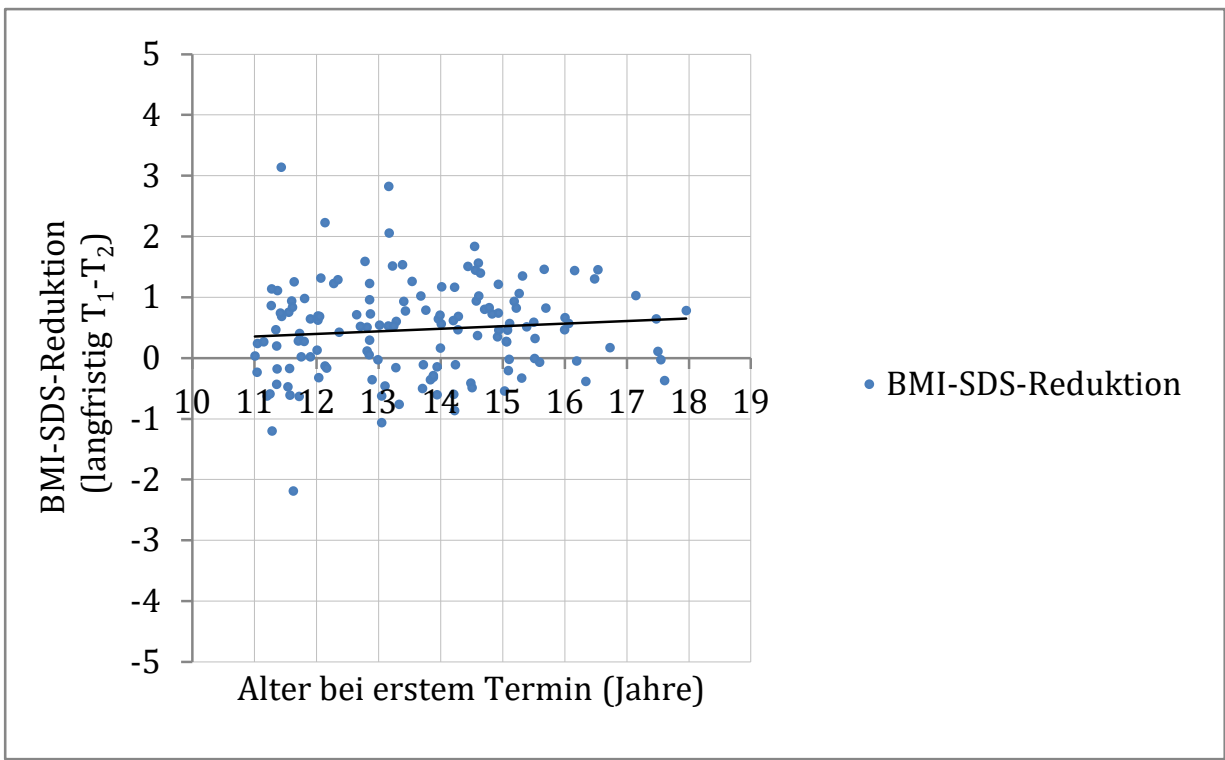

Dagegen zeigte sich sowohl für den langfristigen $\left(\mathrm{r}^{2}=\right.$ 0,$12 ; \quad p=0,17$ Abbildung 24) wie auch für den Gesamtverlauf $\left(\mathrm{r}^{2}\right.$ $=0,09 ; \mathrm{p}=0,3)$

eine signifikante Positivkorrelation. Da letzterer Zusammenhang in einer linearen Regression (entsprechend einer Korrelation nach Bravais und Pearson) nicht darstellbar war, zeigt Abbildung 25 die entsprechende lineare Regression der Rangkorrelation über den Gesamtverlauf.

Während also für den Therapiezeitraum selbst ein jüngeres Alter mit einer größeren Gewichtsreduktion assoziiert war, kam es langfristig zu einer Umkehrung und höhere Altersgruppen zeigten sich erfolgreicher.

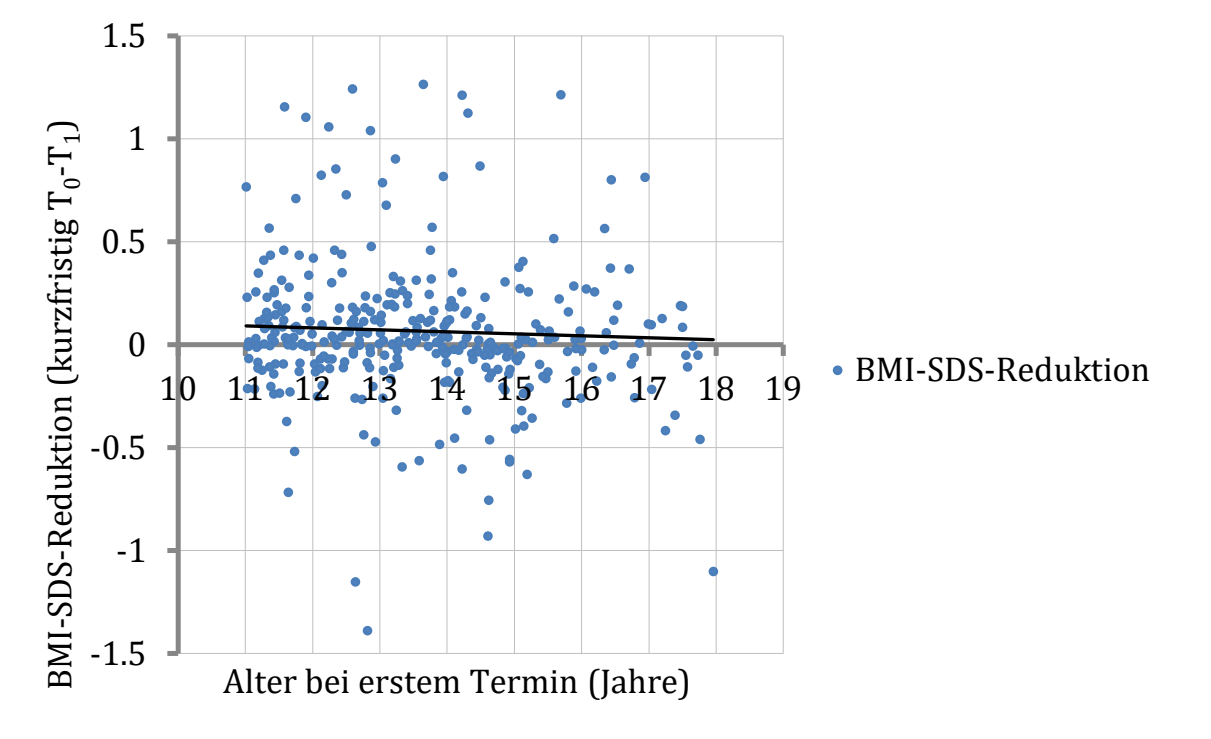

Abbildung 24: Lineare

Regression mit Auftragung der langfristigen BMI-SDSReduktion gegen das Alter bei Beginn der Therapie 
Abbildung 25: Lineare Regression mit Auftragung der Rangkorrelation der BMI-SDS-Reduktion über den Gesamtverlauf gegen das Alter bei Therapiebeginn

Weiterhin erfolgte eine Untersuchung des Zusammenhangs zwischen dem Alter bei Therapiebeginn und anderen für die BMI-SDS-Reduktion relevanten Parametern (Tabellen 26 und 27). Ein höheres Alter bei Therapiebeginn ist dabei mit einem höheren BMI-SDS bei Therapiebeginn sowie einer geringeren Steigerung der körperlichen Aktivität assoziiert.

Für die Parameter „Therapiekonzept“, „Steigerung der Aktivität im Sportverein“, „Profit von ärztlicher Betreuung“, „Zufriedenheit mit Ernährungsberatung“, „Profit durch Ernährungsberatung", „Vorhandensein eines konkreten Zielgewichts vor Therapiebeginn“, „Geschlecht“, „Vorliegen einer Insulinresistenz“, „Erhalt zusätzlicher kinder- und jugendpsychiatrischer Therapie“ sowie „abgeschlossene Berufsausbildung“ konnte kein Zusammenhang zum Alter bei Therapiebeginn erhoben werden.

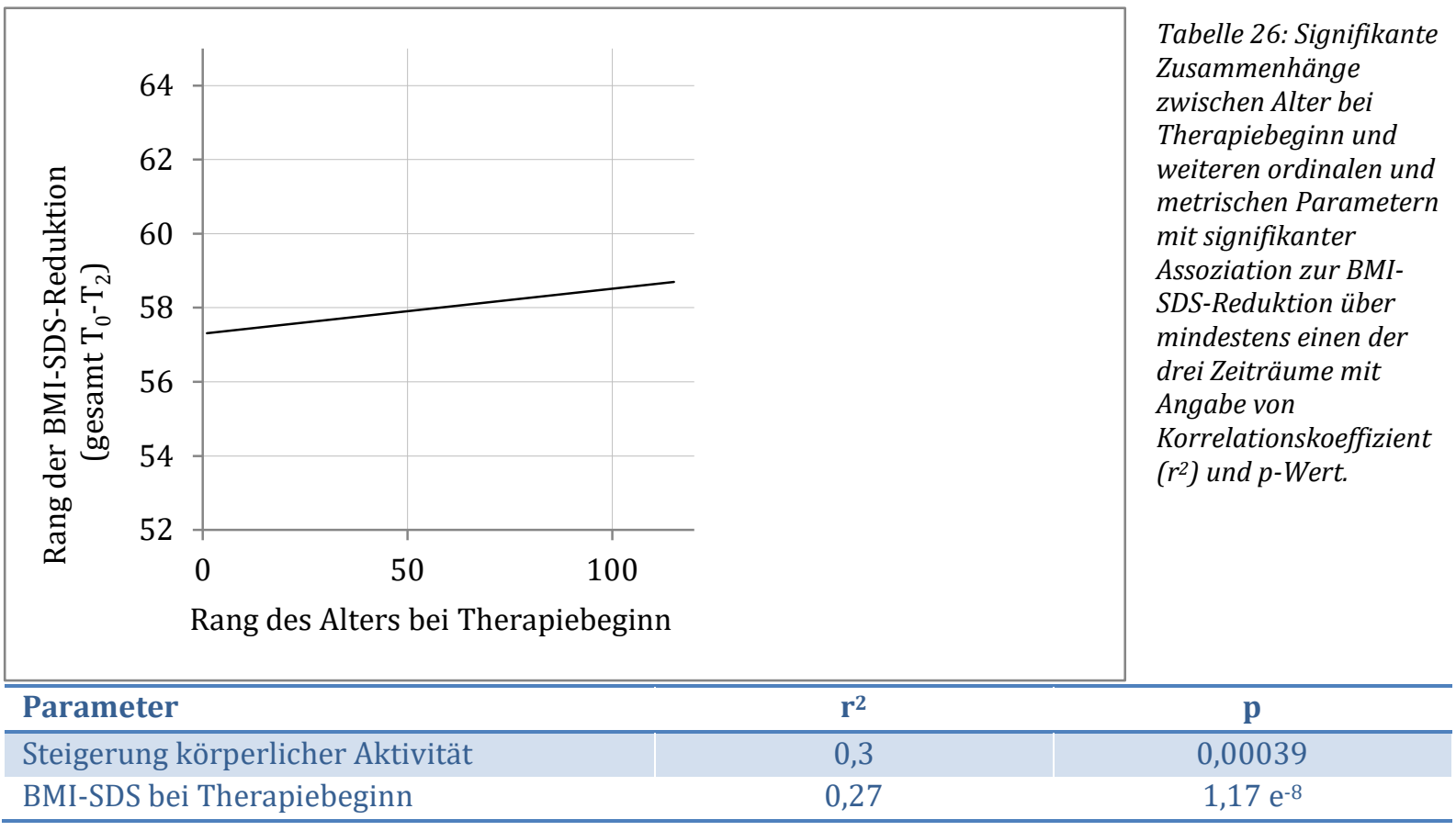

Tabelle 27: Signifikanter Zusammenhang zwischen Alter bei Therapiebeginn und dem nominalen Parameter „Mitgliedschaft im Sportverein“ mit Angabe des jeweiligen Durchschnittsalters sowie des entsprechenden p-Werts.

\begin{tabular}{c|c|c|c}
\hline Parameter & \multicolumn{2}{c}{ Durschnittsalter \pm SD } & p \\
\hline Mitgliedschaft im Sportverein & ja & nein & \\
\hline
\end{tabular}




\subsubsection{BMI-SDS bei Therapiebeginn}

Weiterhin konnten Zusammenhänge zwischen den BMI-SDS-Werten zum Zeitpunkt des Therapiebeginns und der Gewichtsreduktion über die verschiedenen Zeiträume ermittelt werden.

Über den Therapiezeitraum bestand hierbei eine signifikante Negativkorrelation $\left(r^{2}=-0,11 ; p\right.$ $<0,05$; Abbildung 26). Ein höherer BMI-SDS zu Therapiebeginn war also mit einer geringeren Gewichtsreduktion assoziiert. Dieser Trend ließ sich langfristig in geringerem Umfang ebenfalls darstellen $\left(r^{2}=0,07\right)$. Über den gesamten Zeitraum konnte dagegen nur eine sehr geringe Korrelation erfasst werden $\left(\mathrm{r}^{2}=0,02\right)$. Die Ergebnisse für die Zusammenhänge mit der langfristigen und gesamten BMI-SDS-Reduktion waren jedoch bei p-Werte $>0,05$ nicht signifikant.

Abbildung 26: Lineare Regression mit Auftragung der kurzfristigen BMI-SDS-Reduktion gegen den BMI-SDS beim ersten Termin

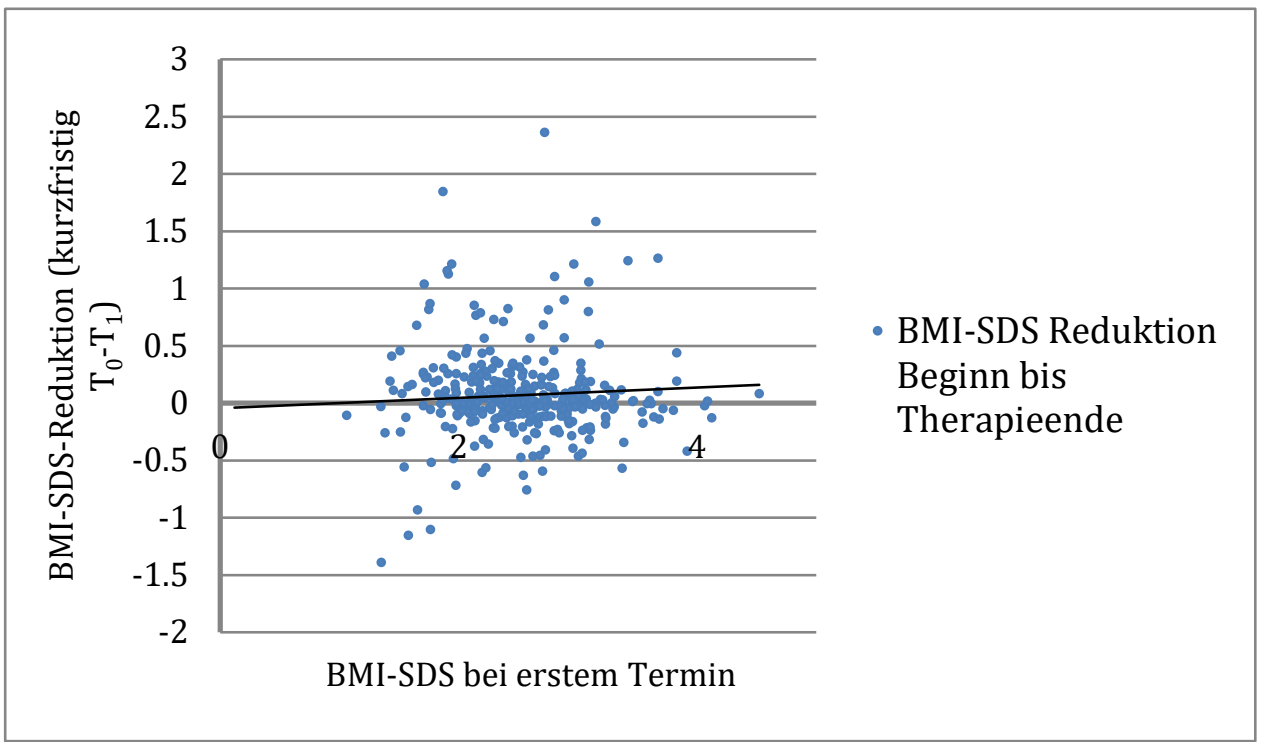

Bei der Untersuchung des BMI-SDS zu Therapiebeginn im Zusammenhang mit den übrigen für die BMI-SDSReduktion relevanten Parametern fiel ein

Zusammenhang zum Alter beim ersten Termin auf (siehe Kapitel

"Alter bei Therapiebeginn“; $r^{2}=0,27 ; p=1,17 \mathrm{e}^{-8}$ ). $\mathrm{Zu}$ den Parametern „Steigerung der körperlichen Aktivität“, „Steigerung der Aktivität im Sportverein“, „Zufriedenheit mit ärztlicher Betreuung“, „Zufriedenheit mit Ernährungsberatung“, „Profit durch Ernährungsberatung“, „Geschlecht", „Vorliegen einer Insulinresistenz“, „Erhalt zusätzlicher kinder- und jugendpsychiatrischer Therapie“, „Abgeschlossene Berufsausbildung“ sowie „Mitgliedschaft im Sportverein" konnte keine signifikante Assoziation dargestellt werden.

\subsubsection{Geschlecht}

Für das Geschlecht der Patienten konnte über alle drei Zeiträume eine signifikant bessere Gewichtsreduktion für Jungen im Vergleich zu Mädchen festgestellt werden (Abbildung 27). 
Über den Therapiezeitraum erreichten Jungen eine um 0,10 (KI: $\pm 0,08$ ) größere BMI-SDSReduktion als Mädchen $(p=0,02)$. Langfristig liegt die Differenz bei 0,09 (KI: $\pm 0,26 ; p=0,49)$ und über den gesamten Verlauf bei 0,21 (KI: $\pm 0,28 ; p=0,13$ ).

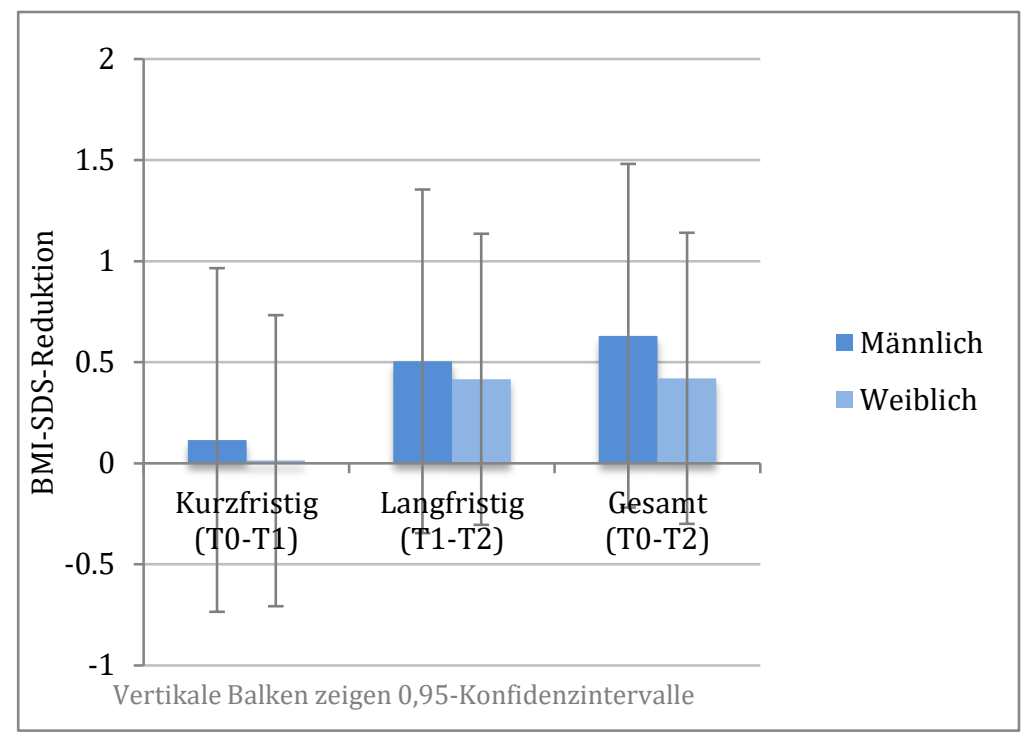

Abbildung 27: Vergleichende Darstellung der kurzfristigen, langfristigen und gesamten BMI-SDS-Reduktion bei Jungen und Mädchen

Auch an dieser Stelle erfolgte eine Überprüfung der Zusammenhänge des Geschlechts zu den übrigen Parametern mit Assoziation zur BMI-SDS-Reduktion über mindestens einen der drei Zeiträume (Tabelle 28). Es fällt auf, dass männliche Patienten im Vergleich zu weiblichen bei Therapiebeginn signifikant jünger waren sowie einen signifikant niedrigeren BMI aufwiesen. Weiterhin lag bei Jungen im Vergleich zu Mädchen seltener eine Insulinresistenz vor.

Tabelle 28: Signifikante Zusammenhänge zwischen Patientengeschlecht und weiteren Parametern mit signifikanter Assoziation zur BMI-SDS-Reduktion über mindestens einen der drei Zeiträume unter Angabe des p-Werts.

\begin{tabular}{l|c|c|c}
\hline Parameter & Männlich & Weiblich & p \\
\hline Alter bei Therapiebeginn & \multicolumn{3}{c}{ Durchschnittswert \pm KI } \\
\hline BMI-SDS bei Therapiebeginn & $13,40 \pm 0,22$ & $13,94 \pm 0,26$ & 0,001 \\
\hline Vorliegen einer Insulinresistenz: & $2,34 \pm 0,06$ & $2,27 \pm 0,08$ & $<0,0001$ \\
\hline Ja & \multicolumn{3}{c}{ Anzahl (n) } \\
\hline Nein & 22 & 43 & 0,0004 \\
\hline
\end{tabular}

Es ließ sich kein Zusammenhang des Geschlechts zu den Parametern „Therapiekonzept", „Steigerung der körperlichen Aktivität", "Steigerung der Aktivität im Sportverein“, „Zufriedenheit mit ärztlicher Betreuung“, „Zufriedenheit mit Ernährungsberatung“, „Profit durch Ernährungsberatung“, „Vorhandensein eines konkreten Zielgewichts vor Therapiebeginn“, „Erhalt zusätzlicher kinder- und jugendpsychiatrischer Therapie“, „Abgeschlossene Berufsausbildung“ sowie „Mitgliedschaft im Sportverein“ erheben.

\subsubsection{Diagnosen und Komorbiditäten}

Tabelle 29 gibt eine Übersicht über die häufigsten erfassten Diagnosen der in die Untersuchung eingegangenen Patienten. Diese Diagnosen wurden auf Zusammenhänge mit der BMI-SDSReduktion über die verschiedenen Zeiträume untersucht. 
Insgesamt lagen bei $74 \%$ (265) der Patienten adipositasbezogene Komorbiditäten vor. Psychiatrische Diagnosen wurden hierbei nicht mit eingerechnet, da die jeweiligen Diagnosen keine Rückschlüsse darüber zuließen, ob die Erkrankung tatsächlich auf die Adipositas zurückzuführen war.

Tabelle 29: Darstellung der häufigsten Diagnosen der eingegangenen Patienten

\begin{tabular}{lc}
\hline Metabolische Veränderungen & $\begin{array}{c}\text { Prozent der Patienten } \\
\text { (absolute Zahlen) }\end{array}$ \\
\hline Hyperurikämie & $16,8 \%(60)$ \\
\hline Insulinresistenz und pathologische Glukosetoleranz & $9,8 \%(35)$ \\
\hline Dyslipidämie & $9,5 \%(34)$ \\
\hline Arterieller Hypertonus & $9,2 \%(33)$ \\
\hline Psychiatrische Diagnosen & \\
\hline Emotionale Störung & $14 \%(50)$ \\
\hline Psychologische Faktoren bei andernorts & $5,6 \%(20)$ \\
klassifizierten Erkrankungen & \\
\hline Störung des Sozialverhaltens & $4,2 \%(15)$ \\
\hline ADS / ADHS & $3,9 \%(14)$ \\
\hline Depression / depressive Episode & $3,9 \%(14)$ \\
\hline Belastungsreaktion & $3,4 \%(12)$ \\
\hline Anpassungsstörung & $2,8 \%(10)$ \\
\hline Sonstige Diagnosen & \\
\hline Eingeschränkte Lern- und Leistungsmöglichkeiten & $3,9 \%(32)$ \\
\hline Genu valgum & $5,3 \%(19)$ \\
\hline Asthma bronchiale & $3,9 \%(14)$ \\
\hline Hypothyreose & \\
\hline
\end{tabular}

Bei den häufigsten Diagnosen handelte es sich mit 38,5 \% (136 Patienten) um metabolische Veränderungen. Hierzu zählt unter anderem die Insulinresistenz bzw. pathologische Glukosetoleranz (35 Patienten, 9,8 \%). Für das Vorliegen einer Insulinresistenz konnte dabei ein Zusammenhang mit dem Erfolg in der Gewichtsreduktion festgestellt werden (Abbildung 28). So erreichten über den Therapieverlauf Patienten ohne Insulinresistenz eine im Schnitt um 0,16 (KI: $\pm 0,12 ; p=0,005$ ) signifikant höhere BMI-SDS-Reduktion. Langfristig ließ sich dieser Zusammenhang jedoch nicht mehr darstellen.

Es erfolgte ebenfalls eine Untersuchung auf Zusammenhänge zwischen dem Vorliegen einer Insulinresistenz und weiteren Parametern mit Assoziation zur BMI-SDS-Reduktion (Tabelle 30). Eine Insulinresistenz hing dabei mit einem höheren Ausgangs-BMI zusammen und lag signifikant häufiger bei weiblichen als bei männlichen Patienten vor. Weiterhin erhielten Patienten mit Vorliegen einer Insulinresistenz ein signifikant intensiveres Therapiekonzept.

Für die Parameter „Steigerung der körperlichen Aktivität“, „Steigerung der Aktivität im Sportverein“, „Zufriedenheit mit ärztlicher Betreuung“, „Zufriedenheit mit Ernährungsberatung“, „Profit durch Ernährungsberatung", „Vorhandensein eines konkreten Zielgewichts vor Therapiebeginn“, „Alter bei Therapiebeginn“, „Erhalt zusätzlicher kinder- und jugendpsychiatrischer Therapie“, „Abgeschlossene Berufsausbildung“ sowie „Mitgliedschaft im Sportverein" konnte keine signifikante Assoziation zum Vorliegen einer Insulinresistenz festgestellt werden.

Abbildung 28: Vergleichende Darstellung der BMI-SDS-Reduktion über den Therapiezeitraum von Patienten mit bzw. ohne Vorliegen einer Insulinresistenz 


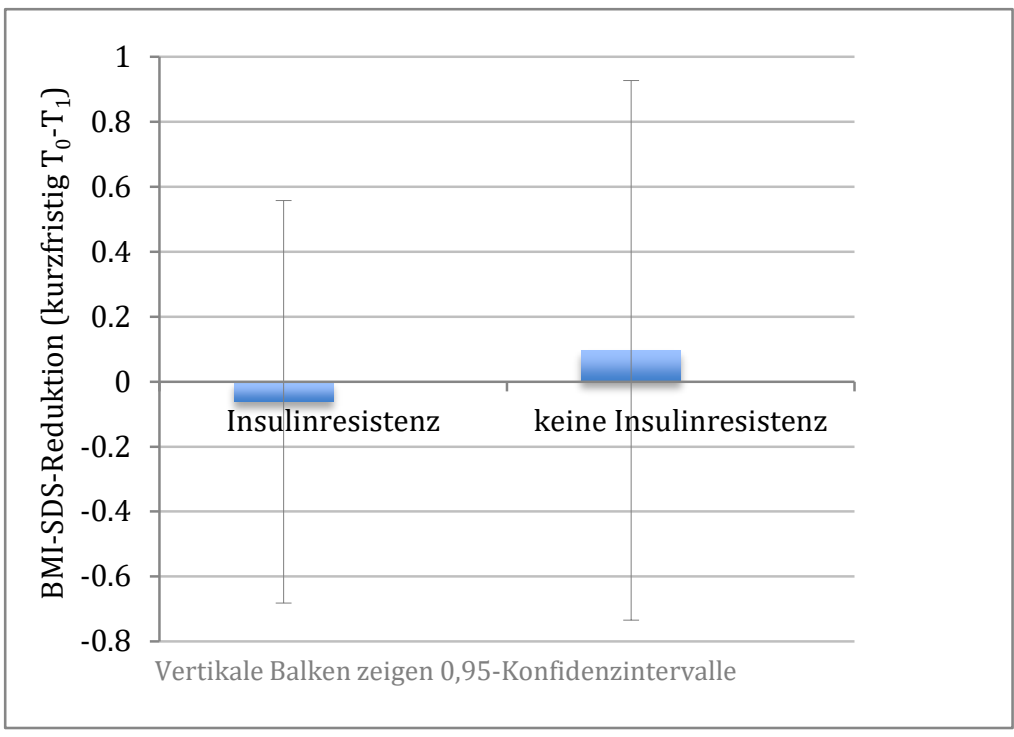

Tabelle 30: Signifikante Zusammenhänge zwischen Vorliegen einer Insulinresistenz und weiteren metrischen und nominalen Parametern mit signifikanter Assoziation zur BMI-SDSReduktion über mindestens einen der drei Zeiträume unter Angabe des p-Werts.

\begin{tabular}{|c|c|c|c|}
\hline Parameter & $\begin{array}{c}\text { Insulinresisten } \\
\mathrm{z}\end{array}$ & $\begin{array}{c}\text { Keine } \\
\text { Insulinresisten } \\
\mathrm{z}\end{array}$ & $\mathbf{p}$ \\
\hline & \multicolumn{2}{|c|}{ Durchschnittswert \pm SD } & \\
\hline BMI-SDS bei Therapiebeginn & $2,82 \pm 0,12$ & $2,43 \pm 0,06$ & $<0,0001$ \\
\hline Therapiekonzept & \multicolumn{2}{|c|}{-} & 0,003 \\
\hline Geschlecht: & \multicolumn{2}{|c|}{ Anzahl (n) } & \\
\hline Männlich & 22 & 212 & \multirow{2}{*}{0,0004} \\
\hline Weiblich & 43 & 155 & \\
\hline
\end{tabular}

Die insgesamt zweithäufigste Diagnose bei $14 \%$ (50) der Patienten war eine emotionale Störung im Kindes- und Jugendalter. Auch generell lagen bei einem großen Teil der Patienten (36 \%, 129 Patienten) psychiatrische Diagnosen vor. Das Vorliegen einer psychiatrischen Diagnose war mit einer vergleichsweise geringeren Gewichtsreduktion assoziiert (Abbildung 29). Kurzfristig erreichten Kinder und Jugendliche ohne psychiatrische Diagnose eine nur minimal bessere BMI-SDS-Reduktion (Unterschied 0,01; KI: \pm 0,08; $\mathrm{p}=0,8$ ), dieses Ergebnis ist jedoch insbesondere mit Rücksicht auf den hohen p-Wert wenig aussagekräftig. Langfristig wurde jedoch eine im Schnitt um 0,24 (KI: $\pm 0,28 ; p=0,09$ ) höhere BMI-SDS-Reduktion erreicht. Über den gesamten Therapiezeitraum konnte durch diese Patienten eine um 0,05 (KI: $\pm 0,08 ; p=0,28$ ) höhere BMI-SDS-Reduktion erreicht werden. Dennoch liegt auch hier der pWert über dem Signifikanzniveau von 0,05, sodass kein signifikanter Zusammenhang vorliegt.

Ein Viertel der Kinder (86 Patienten) erhielten neben der psychologischen Unterstützung im Rahmen der Adipositastherapie zusätzliche kinder- und jugendpsychiatrische Interventionen. Es konnte ein Zusammenhang zwischen dem Erhalt anderweitiger psychiatrischer Interventionen und der BMI-SDS-Reduktion festgestellt werden (Abbildung 30). So zeigte sich über den Therapieverlauf eine signifikant bessere Abnahme mit einem Unterschied von 0,11 (KI: $\pm 0,10 ; p$ $=0,02$ ) bei Kindern und Jugendlichen, die keine weitere psychiatrische Therapie erhalten hatten. Dieser Trend ließ sich jedoch langfristig und auch über den gesamten Untersuchungsverlauf nicht mehr darstellen. 

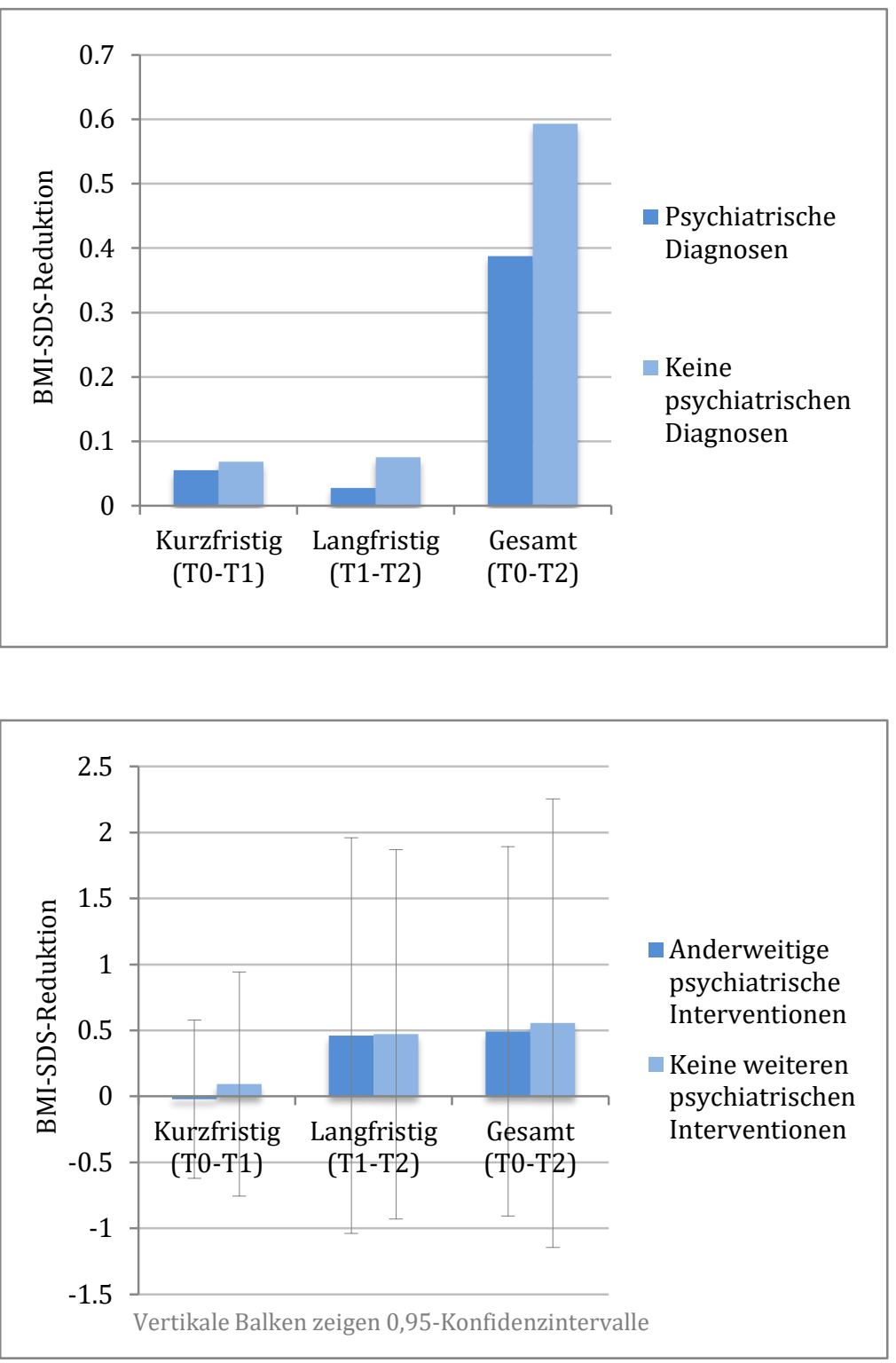

Abbildung 29: Vergleichende Darstellung der durchschnittlichen kurzfristigen, langfristigen und gesamten BMI-SDSReduktion für Patienten mit bzw. ohne psychiatrische Diagnosen

Abbildung 30: Durchschnittlich erreichte kurzfristige, langfristige und gesamte BMI-SDS-Reduktion von Patienten mit bzw. ohne psychiatrische Interventionen zusätzlich zur Adipositastherapie

Auch an dieser Stelle erfolgte eine Überprüfung auf Zusammenhänge zwischen dem Erhalt zusätzlicher psychiatrischer Interventionen und den übrigen Parametern mit Assoziation zur BMI-SDS-Reduktion (Tabelle 31). Dabei fiel auf, dass Patienten mit zusätzlichem Erhalt einer psychiatrischen Therapie bei Therapiebeginn signifikant älter waren, höhere BMI-SDS aufwiesen und im Rahmen der Therapie ihre körperliche Aktivität nur in geringerem Ausmaß steigerten.

Tabelle 31: Signifikante Zusammenhänge zwischen dem Erhalt zusätzlicher psychiatrischer Interventionen und weiteren Parametern mit signifikanter Assoziation zur BMI-SDS-Reduktion über mindestens einen der drei Zeiträume unter Angabe des p-Werts. Die Angaben "gleich" und "häufiger" beziehen sich auf die durchschnittlichen Patientenangaben bezüglich der Entwicklung ihrer körperlichen Aktivität durch die Therapie.

\begin{tabular}{|c|c|c|c|}
\hline Parameter & $\begin{array}{c}\text { Psychiatrische } \\
\text { Therapie }\end{array}$ & $\begin{array}{c}\text { Keine } \\
\text { psychiatrische } \\
\text { Therapie }\end{array}$ & p \\
\hline & \multicolumn{3}{|c|}{ Durchschnittswert \pm SD } \\
\hline Steigerung körperliche Aktivität & „gleich" & „häufiger“ & 0,04 \\
\hline Alter bei Therapiebeginn & $14,05 \pm 0,19$ & $13,47 \pm 0,93$ & 0,003 \\
\hline
\end{tabular}


Es konnte kein Zusammenhang zwischen dem Erhalt zusätzlicher psychiatrischer Interventionen und den Parametern „Therapiekonzept", „Steigerung der Aktivität im Sportverein“, „Zufriedenheit mit ärztlicher Betreuung“, „Zufriedenheit mit Ernährungsberatung“, „Profit durch Ernährungsberatung“, „Vorhandensein eines konkreten Zielgewichts vor Therapiebeginn", "Geschlecht", „Vorliegen einer Insulinresistenz", „Abgeschlossene Berufsausbildung“ sowie „Mitgliedschaft im Sportverein“ erhoben werden.

Für die übrigen Diagnosen ließ sich keine Assoziation zur BMI-SDS-Reduktion erfassen.

\subsubsection{Familiäre Situation und SES}

\subsubsection{Schulbildung und berufliche Situation des Patienten}

Auch in Bezug auf die Familiensituation und den SES der Patienten konnten Zusammenhänge zur BMI-SDS-Reduktion über die verschiedenen Zeiträume festgestellt werden.

Zum einen konnte eine Assoziation zwischen der erreichten Gewichtsreduktion und der Schulbildung des Patienten selbst festgestellt werden (Abbildung 31). Zum Follow-up-Zeitpunkt gaben 3,1 \% der befragten Patienten an, keinen Schulabschluss zu haben (ausgenommen sind hierbei Patienten, die sich zum Zeitpunkt der Befragung noch in der Schulausbildung befanden). Weitere 8,6 \% der Patienten gaben einen Hauptschulabschluss, 24,2 \% einen Realschulabschluss (davon 22,6 \% mit Fachhochschulreife) und 5,5 \% Abitur als Schulabschluss an. Ein Anteil von 43,8 \% der Patienten hatte zum Zeitpunkt der Befragung die Schulausbildung noch nicht beendet, 14,8 \% machten diesbezüglich keine Angaben.

Über den Therapiezeitraum erreichten Patienten, die keinen Schulabschluss angaben, im Vergleich eine um 0,62 (KI: $\pm 0,64 ; p=0,06$ ) geringere BMI-SDS-Reduktion. Bei einem p-Wert von 0,06 liegen die Ergebnisse knapp über dem festgelegten Signifikanzniveau. Auch über den gesamten Zeitraum lässt sich ein Zusammenhang darstellen, wobei Patienten ohne Schulabschluss eine im Schnitt um 0,22 (KI: \pm 0,92; $\mathrm{p}=0,63$ ) geringere BMI-SDS-Reduktion erreichen. Langfristig konnte kein Zusammenhang zwischen einem fehlenden Schulabschluss und einer geringeren Gewichtsreduktion festgestellt werden.

Ähnliche Ergebnisse ergaben sich in Bezug auf die Berufsausbildung des Patienten (Abbildung 32). Zum Zeitpunkt der Follow-Up-Erhebung gaben 3,1 \% der Patienten an, keinen Beruf erlernt zu haben (nicht eingeschlossen sind hierbei Patienten, die sich zum Befragungszeitpunkt noch in der Schul- bzw. Berufsausbildung befanden). Ein Prozentsatz von 1,6 gab eine Lehre, von 28,1 eine Ausbildung und von 6,3 ein Studium an. Die übrigen 17,1 \% der Patienten machten diesbezüglich keine Angaben.

Patienten ohne Berufsausbildung erzielten im Vergleich über den Therapiezeitraum eine um 0,64 (KI: $\pm 0,60 ; p=0,03$ ) signifikant niedrigere BMI-SDS-Reduktion. Über den Gesamtverlauf erreichten Patienten ohne Berufsausbildung eine um 0,61 (KI: $\pm 0,94 ; p=0,2$ ) geringere BMISDS-Reduktion. Langfristig lässt sich nur ein minimaler Unterschied zwischen den beiden Gruppen feststellen, wobei Patienten ohne Schulabschluss eine um 0,12 (KI: \pm 0,92) bessere Reduktion erzielen, dieser Wert liegt jedoch bei einem p-Wert von 0,79 weit über dem Signifikanzniveau.

Eine Überprüfung auf Zusammenhänge zwischen dem Vorliegen einer abgeschlossenen Berufsausbildung und den übrigen Paramatern mit Assoziation zur BMI-SDS-Reduktion ergab keinerlei signifikante Ergebnisse. Damit besteht kein Zusammenhang zu den Parametern „Therapiekonzept“, „Steigerung der körperlichen Aktivität“, „Steigerung der Aktivität im Sportverein“, „Zufriedenheit mit ärztlicher Betreuung“, „Zufriedenheit mit Ernährungsberatung“, „Profit durch Ernährungsberatung“, „Vorhandensein eines konkreten Zielgewichts vor Therapiebeginn“, „Alter bei Therapiebeginn“, „BMI-SDS bei Therapiebeginn“, „Geschlecht”, „Vorliegen einer Insulinresistenz", „Erhalt zusätzlicher kinder- und jugendpsychiatrischer Therapie“, „Abgeschlossene Berufsausbildung“ sowie „Mitgliedschaft im Sportverein“. 


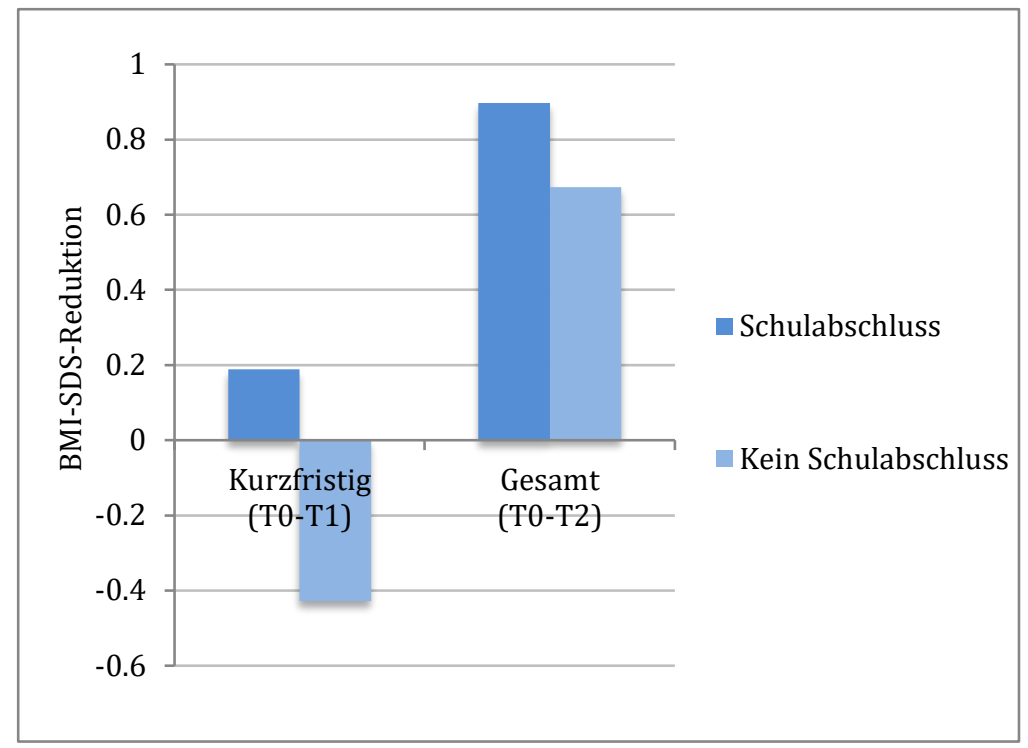

Abbildung 31: Vergleichende Darstellung der kurzfristigen und gesamten durchschnittlichen BMI-SDS-Reduktion der Patienten mit bzw. ohne Schulabschluss zum Zeitpunkt der Follow-up-Erhebung

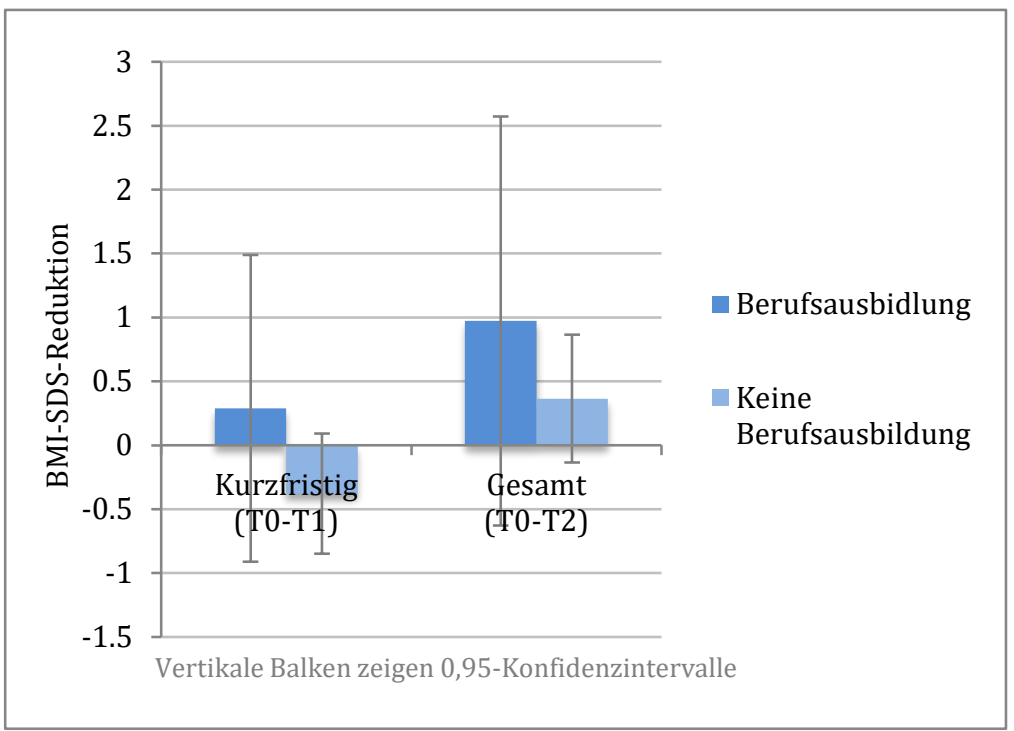

Abbildung 32: Vergleichende Darstellung der kurzfristigen und gesamten durchschnittlichen BMI-SDS-Reduktion von Patienten mit bzw. ohne Berufsausbildung zum Zeitpunkt der Follow-up-Erhebung

\subsubsection{Schulbildung und berufliche Situation der Eltern}

Weiterhin konnten Zusammenhänge zwischen kindlicher Gewichtsreduktion und elterlicher Schul- bzw. Berufsausbildung festgestellt werden.

Von den Müttern der eingegangenen Patienten erreichten 4,7 \% keinen Schulabschluss, $18 \%$ einen Haupt-, 48,4 \% einen Realschulabschluss und $14 \%$ Abitur; für 14,9 \% der Mütter lagen keine Informationen zum Schulabschluss vor. Betrachtet man die Väter der eingegangenen Patienten, so hatten 0,8 \% keine abgeschlossene Schulausbildung, $32 \%$ einen Haupt-, 34,4 \% einen Realschulabschluss und 11,7\% Abitur.

Unter den Müttern schlossen 7,8 \% keine Berufsausbildung ab, 3,1\% eine Lehre, $57 \%$ eine Ausbildung und $7 \%$ ein Studium, für 25,1 \% sind keine Informationen zur Berufsausbildung bekannt. Ein Prozentsatz von 2,3 der Väter der eingegangenen Patienten hatte keine abgeschlossene Berufsausbildung, für 8,6 \% wurde eine Lehre, für 49,2 \% eine Ausbildung und für 7,8 \% ein Studium erfasst. Für 32,1 \% der Väter lagen keine entsprechenden Angaben vor.

Während kein Zusammenhang zwischen elterlichem Schulabschluss und kindlicher Gewichtsreduktion dargestellt werden konnte, zeigten sich Assoziationen zwischen der elterlichen Berufsausbildung und der kindlichen BMI-SDS-Reduktion: Dabei erreichten Patienten, deren Mütter keine Berufsausbildung abgeschlossen hatten, über den 
Therapiezeitraum eine um 0,27 (KI: $\pm 0,38 ; \mathrm{p}=0,16$ ) geringere BMI-SDS-Reduktion. Langfristig konnten ähnliche Zusammenhänge festgestellt werden, der Unterschied zwischen den Gruppen lag hier bei 0,34 (KI: $\pm 0,62 ; p=0,28$ ). Auch über den Gesamtverlauf war eine nicht abgeschlossene mütterliche Berufsausbildung mit einer durchschnittlich um 0,54 geringeren BMI-SDS-Reduktion assoziiert (KI: $\pm 0,70 ; p=0,12$ ) (Abbildung 33). Alle drei Ergebnisse liegen jedoch über dem Signifikanzniveau von 0,05.

Für die Väter der Patienten stellte sich ein ähnlicher Zusammenhang dar (Abbildung 34). Über den Therapiezeitraum erreichten Kinder von Vätern ohne abgeschlossene Berufsausbildung eine um durchschnittlich 0,37 (KI: $\pm 0,35 ; \mathrm{p}=0,29$ ) geringere BMI-SDS-Reduktion. Langfristig wurde eine um 0,3 (KI: $\pm 0,89 ; \mathrm{p}=0,5$ ) niedrigere Reduktion erreicht. Über den Gesamtverlauf lag die BMI-SDS-Reduktion der Kinder von Vätern ohne abgeschlossene Berufsausbildung im

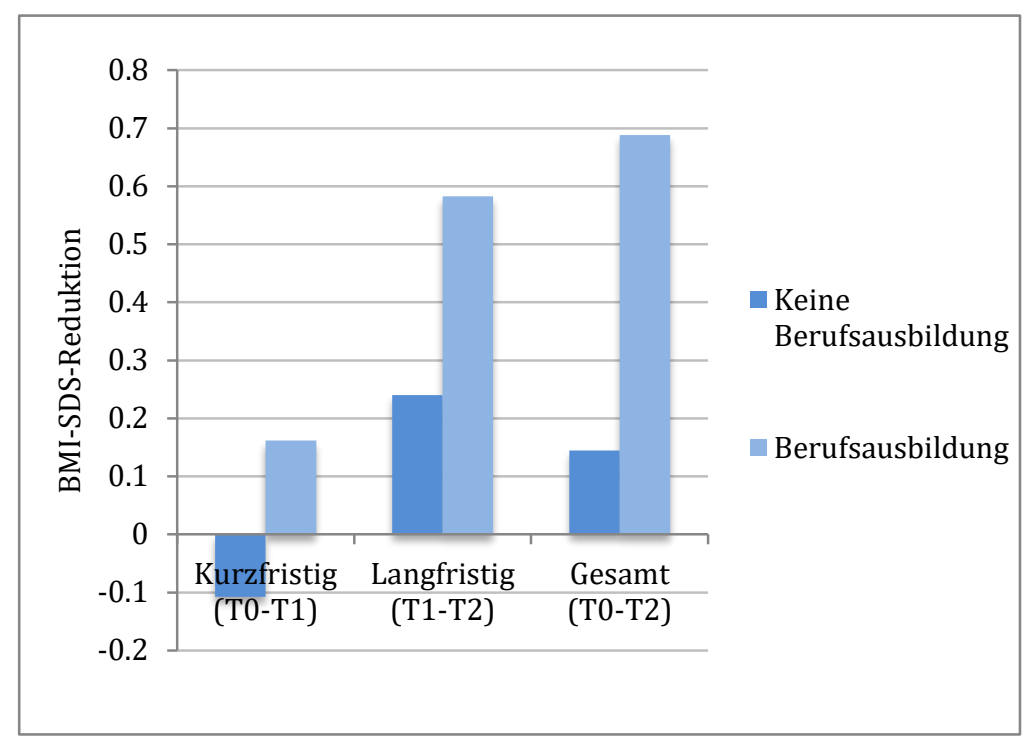

Durchschnitt um 0,65 (KI: \pm $2,04 ; \quad \mathrm{p}=0,2)$ unter der Reduktion der Kinder von Väter mit abgeschlossener Berufsausbildung. Auch diese Ergebnisse liegen jedoch über dem festgelegten Signifikanzniveau.

Abbildung 33: Vergleichende Darstellung der BMI-SDS-Reduktionen über die verschiedenen Zeiträume in Abhängigkeit von der mütterlichen Berufsausbildung

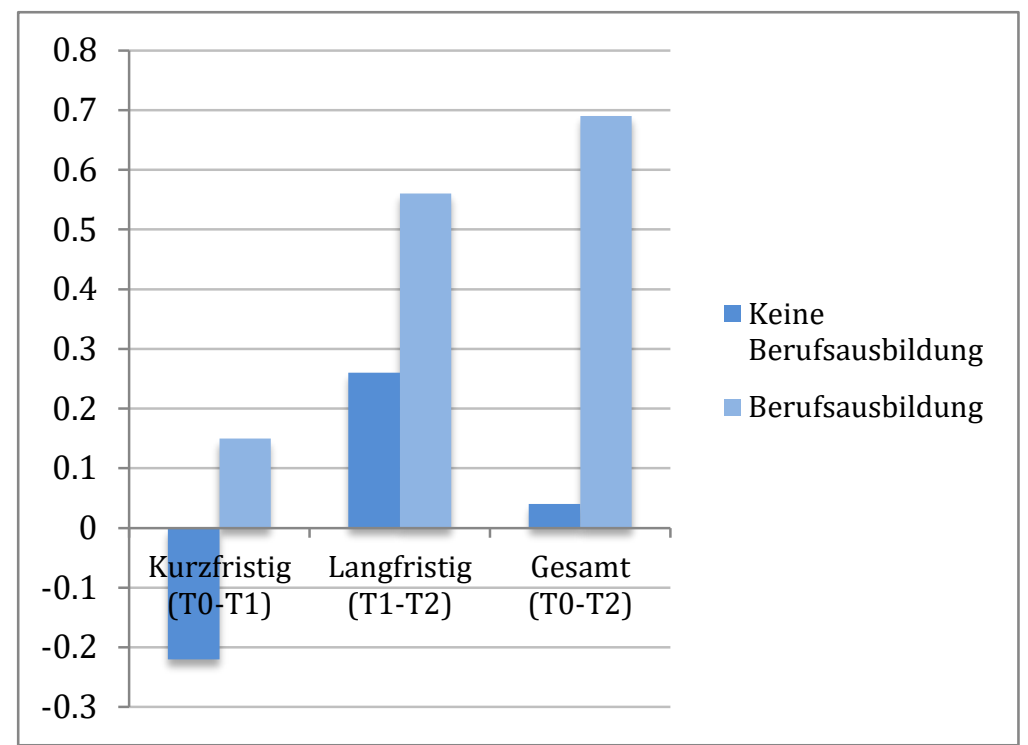

Abbildung 34: Vergleichende Darstellung der BMI-SDS-Reduktionen über die verschiedenen Zeiträume in Abhängigkeit von der väterlichen Berufsausbildung 

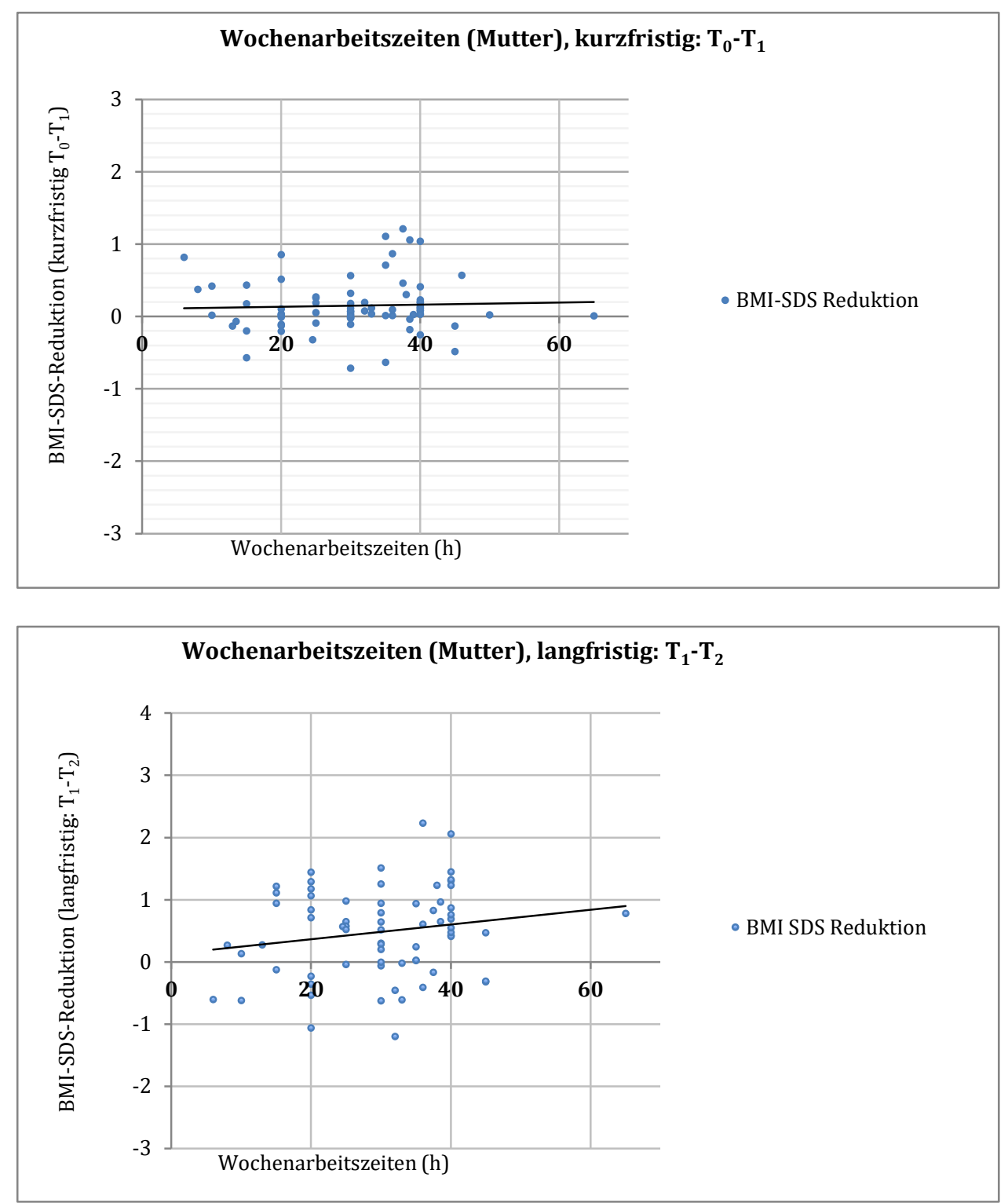

Weiterhin konnte ein Zusammenhang der BMI-SDSReduktion $\mathrm{zu}$ den mütterlichen Wochenarbeitsze iten festgestellt werden

(Abbildungen 35 - 37). Für 63,28 $\%$ der zum Follow-upZeitpunkt befragten Mütter waren die

Wochenarbeitszeiten bekannt. Es ließ sich kurzfristig $\left(r^{2}=0,1 ; p=0,39\right)$, langfristig $\left(r^{2}=0,16\right.$; $\mathrm{p}=0,19)$ und über den Gesamtverlauf $\left(\mathrm{r}^{2}=0,16 ; \mathrm{p}=0,2\right)$ eine Positivkorrelation zwischen der mütterlichen Wochenarbeitszeit und der erreichten Gewichtsreduktion feststellen. Damit sind über alle drei Zeiträume höhere mütterliche Wochenarbeitszeiten mit höheren Gewichtsreduktionen assoziiert, allerdings sind diese Ergebnisse bei p-Werten $>0,05$ nicht signifikant.

Abbildung 35: Lineare Regression mit Darstellung der kurzfristigen BMI-SDS-Reduktion in Abhängigkeit von der mütterlichen Wochenarbeitszeit 
Abbildung 37: Lineare Regression mit Darstellung der BMI-SDS-Reduktion über den Gesamtverlauf in Abhängigkeit von der mütterlichen Wochenarbeitszeit

Für 69,5 \% der Väter der Patienten lagen Angaben bezüglich der Wochenarbeitszeiten vor. Hier ließen sich ähnliche Zusammenhänge, allerdings in geringerem Ausmaß und nur über den Therapiezeitraum $\left(r^{2}=0,06 ; p=0,59\right)$ sowie den Gesamtverlauf $\left(r^{2}=0,08 ; p=0,5\right)$ darstellen. $\mathrm{Zu}$ beachten ist allerdings die recht geringe Aussagekraft dieser Ergebnisse bei relativ hohen pWerten.

\subsubsection{Migrationshintergrund}

Schließlich wurde der Migrationshintergrund als beeinflussender Parameter auf den Erfolg der Gewichtsreduktion untersucht. Es lag für 4,2 \% der Patienten ein einseitiger und für 11,5\% der Patienten ein beidseitiger Migrationshintergrund vor. Damit lag der Gesamtprozentsatz der Patienten mit Migrationshintergrund bei 15,7 \%. Es konnten jedoch für keinen Zeitraum Zusammenhänge oder signifikante Unterschiede für den Erfolg der BMI-SDS-Reduktion von

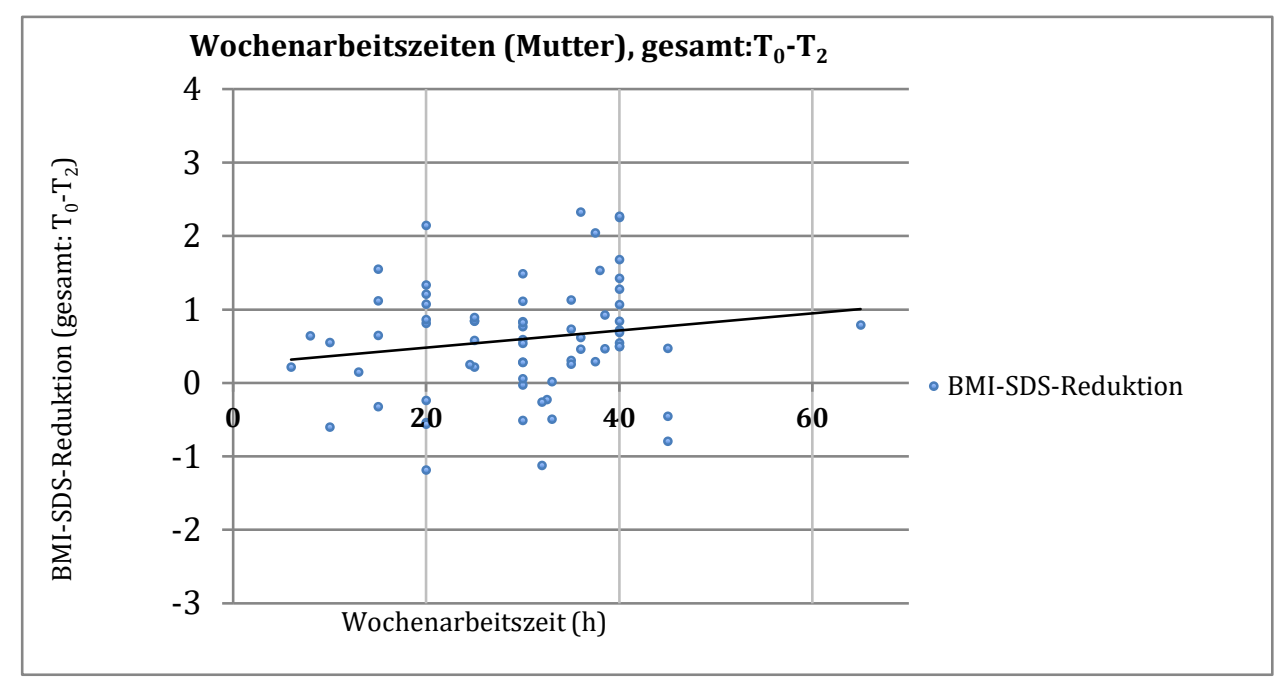

Patienten mit bzw. ohne Migrationshinter grund festgestellt werden.

\subsubsection{Familien \\ - und}

Wohnsituation

Von den

befragten

Patienten

machten 110

Angaben

bezüglich ihrer

Lebens- bzw.

familiären Umstände. In absteigender Häufigkeit lebten die Patienten zusammen mit beiden Eltern (61,8 \%; 68 Angaben), zusammen mit der Mutter (13,6 \%; 15 Angaben), allein (12,7 \%; 14 Angaben), zusammen mit ihrem Vater bzw. in einer Patchwork-Familie (jeweils 4,5\%; 5 Angaben) und in einer Einrichtung (2,7 \%; 3 Angaben). 
Es konnte ein Zusammenhang zwischen der BMI-SDS-Reduktion und den jeweiligen Lebensumständen der Patienten erfasst werden. So erreichten über alle drei Zeiträume Patienten, die in einer Einrichtung (wie z. B. einem Heim) lebten, im Schnitt eine geringere Gewichtsreduktion als Patienten in anderen Wohnsituationen (Abbildung 38). Hierzu zählten „Zusammenleben mit beiden Eltern“, „Zusammenleben mit dem Vater“, „Zusammenleben mit der Mutter“, „Patchwork-Familie“, „Zusammenleben mit Pflegeeltern“ und „alleinlebend“. Über den Therapiezeitraum lag die erreichte BMI-SDS-Reduktion der in Einrichtungen lebenden Patienten um 0,39 (KI: $\pm 0,74 ; p=0,30$ ) unter der Reduktion der Patienten in anderen Wohnsituationen. Langfristig lag der Unterschied bei 0,37 (KI: $\pm 0,88 ; \mathrm{p}=0,40$ ), über den Gesamtverlauf bei 0,67 (KI: $\pm 0,99 ; \mathrm{p}=0,18$ ). Die Ergebnisse sind jedoch bei $\mathrm{p}$-Werten $>0,05$ nicht signifikant.

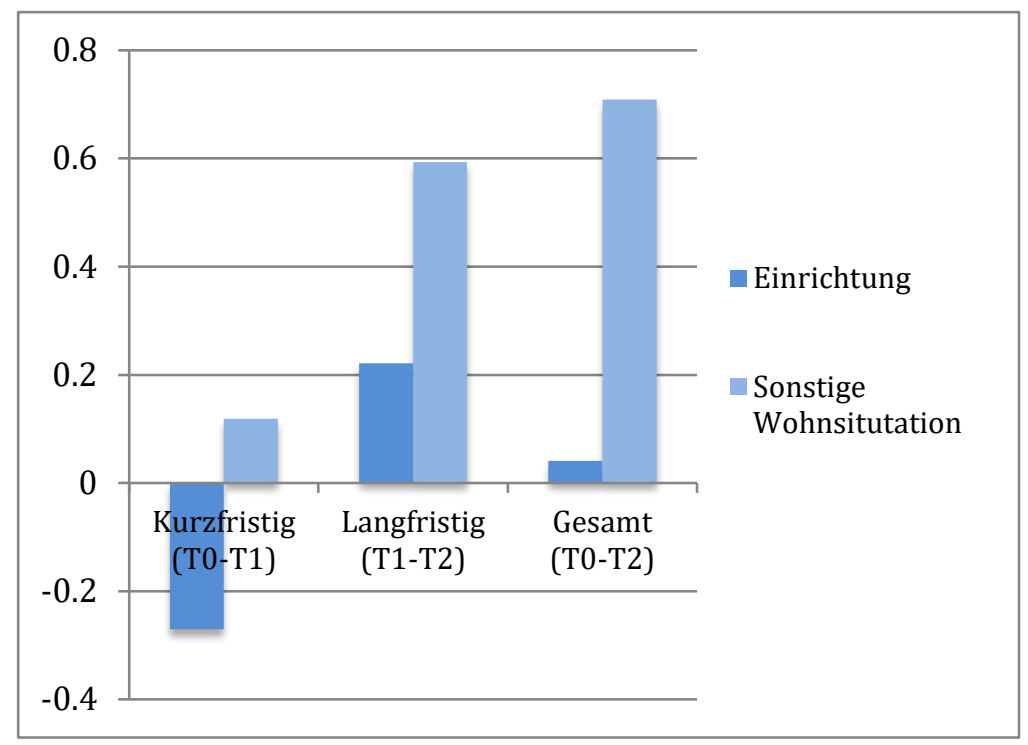

Abbildung 38: Vergleichende Darstellung der kurzfristigen, langfristigen und gesamten BMI-SDS-Reduktion von in Einrichtungen lebenden Patienten im Vergleich zu Patienten in anderen Wohnsituationen

\subsubsection{Elterlicher Gewichtsstatus}

Für 68,7 \% der Mütter der eingegangenen Patienten konnten aus den entsprechenden Akten Gewichts- und Größendaten erhoben werden. Davon waren 19,0 \% normalgewichtig, 51,7 \% übergewichtig und $29,3 \%$ adipös.

Bei den Vätern der Patienten konnten für $62 \%$ Daten für Gewicht und Größe erhoben werden. Von diesen waren 28,0 \% normalgewichtig, 46,9 \% übergewichtig und 25,1\% adipös.

Für den mütterlichen BMI konnte über den Therapiezeitraum ein mäßiger Zusammenhang zur BMI-SDS-Reduktion dargestellt werden $\left(r^{2}=-0,04\right)$. Es handelt sich um eine Negativkorrelation, demnach war ein höheres mütterliches Gewicht mit einer geringeren BMI-SDS-Reduktion assoziiert. Da sich dieser Zusammenhang im Rahmen einer linearen Regression der Werte nicht darstellen ließ, stellt Abbildung 39 eine lineare Regression der entsprechenden Rangkorrelation dar. Bei einem p-Wert von 0,51 liegt dieses Ergebnis jedoch deutlich über dem Signifikanzniveau. Langfristig und über den Gesamtverlauf ließ sich kein Zusammenhang mehr nachweisen. 
Auch für den väterlichen BMI war ein ähnlicher Verlauf darstellbar: Über den Therapiezeitraum bestand eine Negativkorrelation von väterlichen BMI-Werten und BMI-SDS-Reduktion $\left(\mathrm{r}^{2}=\right.$ 0,11; $\mathrm{p}=0,11$; Abbildung 40). Dieses Ergebnis ist jedoch ebenfalls statistisch nicht signifikant. Langfristig und über den Gesamtverlauf konnte keine solche Assoziation mehr dargestellt werden.

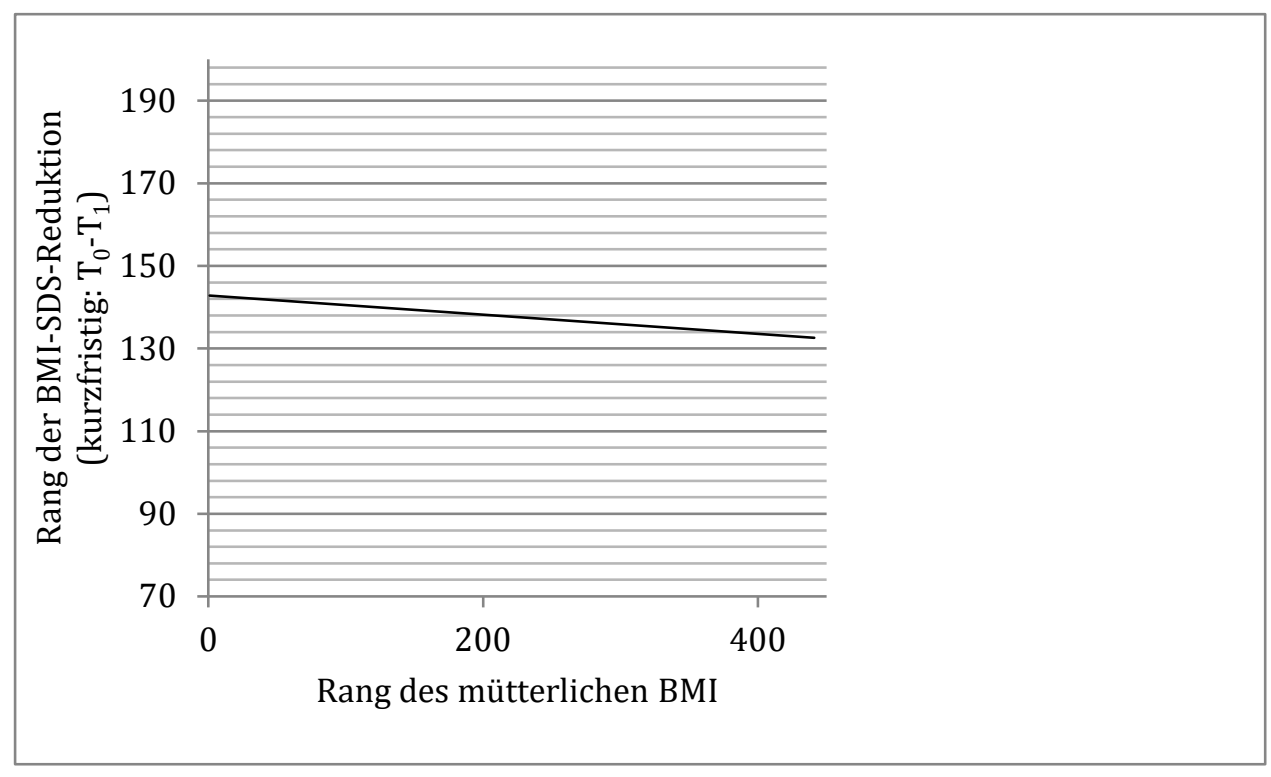

Abbildung 39: Lineare Regression der Rangkorrelation mit Darstellung der kurzfristigen BMISDS-Reduktion in Abhängigkeit vom mütterlichen BMI

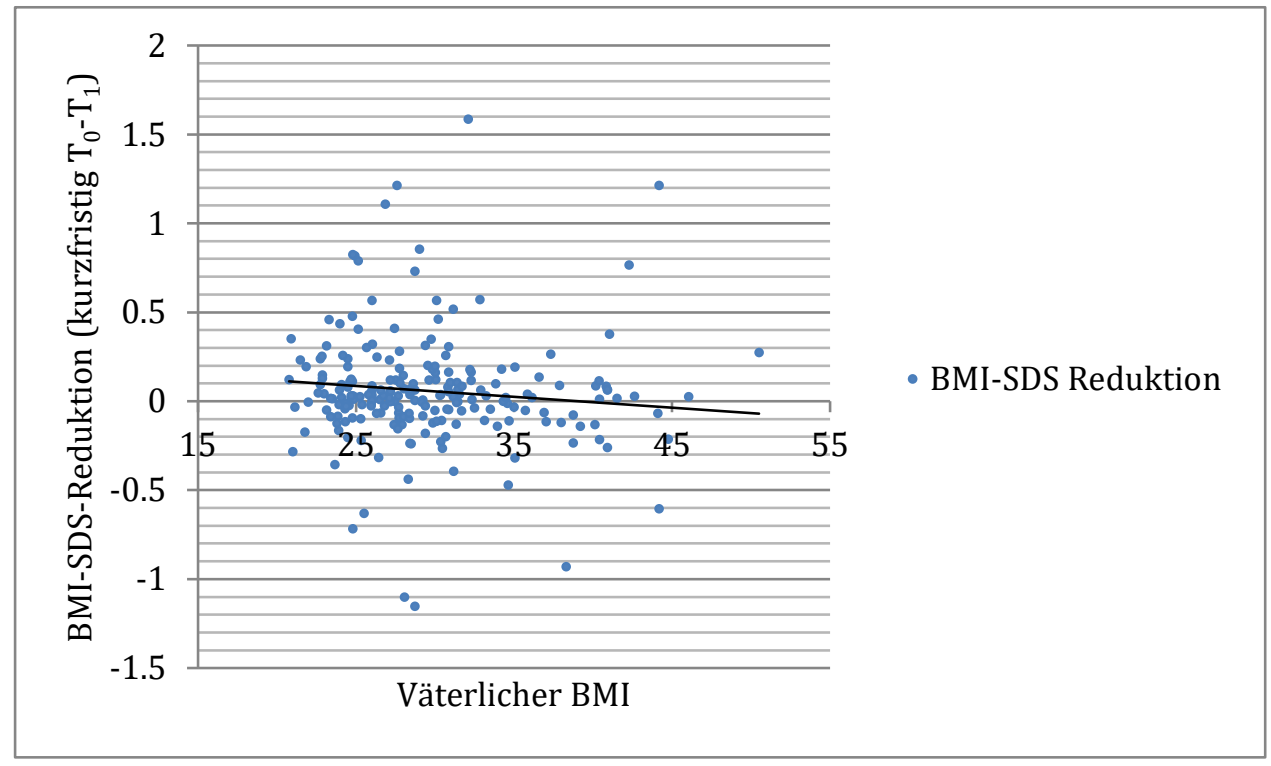

\subsection{Untersuchung des Einflusses sonstiger Parameter auf die BMI-SDS- Reduktion}

Die Parameter „sportliche Aktivität" und „regelmäßiges Wiegen“ ließen sich weder eindeutig der Gruppe der therapieabhängigen noch der der therapieunabhängigen Parameter zuordnen. Grund ist, dass den Patienten im Rahmen der Therapie zwar beide Verhaltensweisen nahegelegt werden, es aber gleichwohl möglich ist, dass sowohl eine sportliche Aktivität als auch ein regelmäßiges Wiegen bereits vor Therapiebeginn (und damit therapieunabhängig) 
vorgenommen wurden. In diesem Sinne werden die Parameter an dieser Stelle separat besprochen.

\subsubsection{Sportliche Aktivität}

Bei Erfassung der sportlichen Aktivität gaben 44,5 \% der Patienten an, um den Therapiezeitraum herum einen Vereinssport ausgeübt zu haben. Im Schnitt wurde die sportliche Aktivität laut Angaben für 8,2 (KI: $\pm 8,8$ ) Stunden pro Monat ausgeübt. Im Vergleich der BMISDS-Reduktionen im Vereinssport tätiger Patienten mit denen von Patienten ohne sportliche Betätigung erreichten über den Gesamtzeitraum erstere eine um 0,39 (KI: $\pm 0,38$ ) signifikant höhere Reduktion ( $\mathrm{p}=0,02$; Abbildung 41). Für die übrigen Zeiträume konnten keine signifikanten Zusammenhänge erhoben werden (siehe Tabelle 32). Die Korrelation der Zeit der Ausübung pro Monat gegen die BMI-SDS-Reduktionen ergab kurzfristig $\left(\mathrm{r}^{2}=0,17\right)$ und über den Gesamtverlauf $\left(\mathrm{r}^{2}=0,16\right)$ eine deutliche Positivkorrelation. Bei einem p-Wert von 0,26 bzw. 0,24 lagen diese Werte jedoch außerhalb des signifikanten Bereichs. Langfristig ließ sich keine Assoziation zur Stundenzahl feststellen.

Ein Satz von 19,5 \% der Patienten gab an, zum Follow-up-Zeitpunkt durchschnittlich für 7,5 (KI: \pm 8 ,9) Stunden pro Monat einen Vereinssport auszuüben. Auch hier ließ sich jedoch kein Zusammenhang zur Gewichtsreduktion sowie zur Stundenzahl der Ausübung darstellen.

Insgesamt 33,6 \% der befragten Patienten führten an, um den Therapiezeitraum herum außerhalb eines Vereins sportlich aktiv gewesen zu sein. Die durchschnittliche Aktivitätsdauer lag bei 9,5 (KI: $\pm 26,2$ ) Stunden im Monat. Zum Follow-up-Zeitpunkt gaben 33,6\% an, eine sportliche Aktivität außerhalb eines Sportvereins mit durchschnittlich 10,4 (KI: $\pm 14,8$ ) Stunden im Monat auszuüben. Für beide Zeiträume ließen sich jedoch keine Zusammenhänge zur kurzfristigen, langfristigen und gesamten BMI-SDS-Reduktion ermitteln. Auch hier konnte keine Assoziation zur Stundenzahl pro Monat dargestellt werden.

Tabelle 32 vergleicht die durchschnittlichen BMI-SDS-Reduktionen der verschiedenen Zeitpunkte der Patienten mit bzw. ohne Ausübung einer sportlichen Aktivität. Auffällig ist, dass Patienten mit Ausübung eines Vereinssports zu beinahe jedem Zeitpunkt (Ausnahme: Ausübung eines Vereinssports zum Follow-up-Zeitpunkt mit Vergleich der langfristigen Reduktionen) höhere durchschnittliche BMI-SDS-Reduktionen aufwiesen als Patienten ohne sportliche Aktivität. Für die Ausübung eines Sports außerhalb eines Vereins ließen sich diese Tendenzen nicht darstellen.

Tabelle 32: Vergleichende Darstellung der erreichten durchschnittlichen BMI-SDS-Reduktionen (unter Angabe des Konfidenzintervalls (KI)) über die verschiedenen Zeiträume von Patienten mit bzw. ohne Ausübung einer sportlichen Aktivität um den Zeitpunkt der Therapie herum bzw. um den Follow-up-Zeitpunkt herum (signifikanter Zusammenhang fett gedruckt)

\begin{tabular}{|c|c|c|c|}
\hline & $\begin{array}{l}\text { BMI-SDS-Reduktion [KI]: } \\
\text { Vereinssport (Therapie) }\end{array}$ & $\begin{array}{l}\text { BMI-SDS-Reduktion [KI]: } \\
\text { kein Sport (Therapie) }\end{array}$ & p-Wert \\
\hline Kurzfristig $\left(\mathrm{T}_{0}-\mathrm{T}_{1}\right)$ & $0,16[ \pm 0,60]$ & $0,12[ \pm 0,76]$ & 0,6 \\
\hline Langfristig $\left(\mathrm{T}_{1}-\mathrm{T}_{2}\right)$ & $0,64[ \pm 1,76]$ & $0,30[ \pm 0,18]$ & 0,06 \\
\hline \multirow[t]{2}{*}{ Gesamt $\left(\mathrm{T}_{0}-\mathrm{T}_{2}\right)$} & $0,75[ \pm 1,86]$ & $0,36[ \pm 1,88]$ & 0,02 \\
\hline & $\begin{array}{l}\text { BMI-SDS-Reduktion [KI]: } \\
\text { Vereinssport (Follow-up) }\end{array}$ & $\begin{array}{l}\text { BMI-SDS-Reduktion [KI]: } \\
\text { kein Sport (Follow-up) }\end{array}$ & p-Wert \\
\hline Kurzfristig $\left(\mathrm{T}_{0}-\mathrm{T}_{1}\right)$ & $0,13[ \pm 0,84]$ & $0,08[ \pm 0,86]$ & 0,6 \\
\hline Langfristig $\left(\mathrm{T}_{1}-\mathrm{T}_{2}\right)$ & $0,35[ \pm 2,34]$ & $0,67[ \pm 1,52]$ & 0,19 \\
\hline Gesamt $\left(\mathrm{T}_{0}-\mathrm{T}_{2}\right)$ & $0,71[ \pm 2,42]$ & $0,45[ \pm 1,78]$ & 0,12 \\
\hline & BMI-SDS-Reduktion [KI]: & BMI-SDS-Reduktion [KI]: & p-Wert \\
\hline
\end{tabular}




\begin{tabular}{|c|c|c|c|}
\hline & Sonstiger Sport (Therapie) & kein Sport (Therapie) & \\
\hline Kurzfristig $\left(\mathrm{T}_{0}-\mathrm{T}_{1}\right)$ & $0,02[ \pm 1,50]$ & $0,12[ \pm 0,76]$ & 0,43 \\
\hline Langfristig $\left(\mathrm{T}_{1}-\mathrm{T}_{2}\right)$ & $0,52[ \pm 1,22]$ & $0,30[ \pm 0,18]$ & 0,29 \\
\hline Gesamt $\left(\mathrm{T}_{0}-\mathrm{T}_{2}\right)$ & $0,61[ \pm 1,72]$ & $0,36[ \pm 1,88]$ & 0,26 \\
\hline
\end{tabular}

\begin{tabular}{lccc} 
& $\begin{array}{c}\text { BMI-SDS-Reduktion [KI]: } \\
\text { Sonstiger Sport (Follow-up) }\end{array}$ & $\begin{array}{c}\text { BMI-SDS-Reduktion [KI]: } \\
\text { kein Sport (Follow-up) }\end{array}$ & p-Wert \\
\hline Kurzfristig $\left(\mathrm{T}_{0}-\mathrm{T}_{1}\right)$ & $0,07[ \pm 0,80]$ & $0,08[ \pm 0,86]$ & 0,88 \\
Langfristig $\left(\mathrm{T}_{1}-\mathrm{T}_{2}\right)$ & $0,66[ \pm 1,44]$ & $0,67[ \pm 1,52]$ & 0,97 \\
Gesamt $\left(\mathrm{T}_{0}-\mathrm{T}_{2}\right)$ & $0,46[ \pm 1,56]$ & $0,45[ \pm 1,78]$ & 0,95 \\
\hline
\end{tabular}

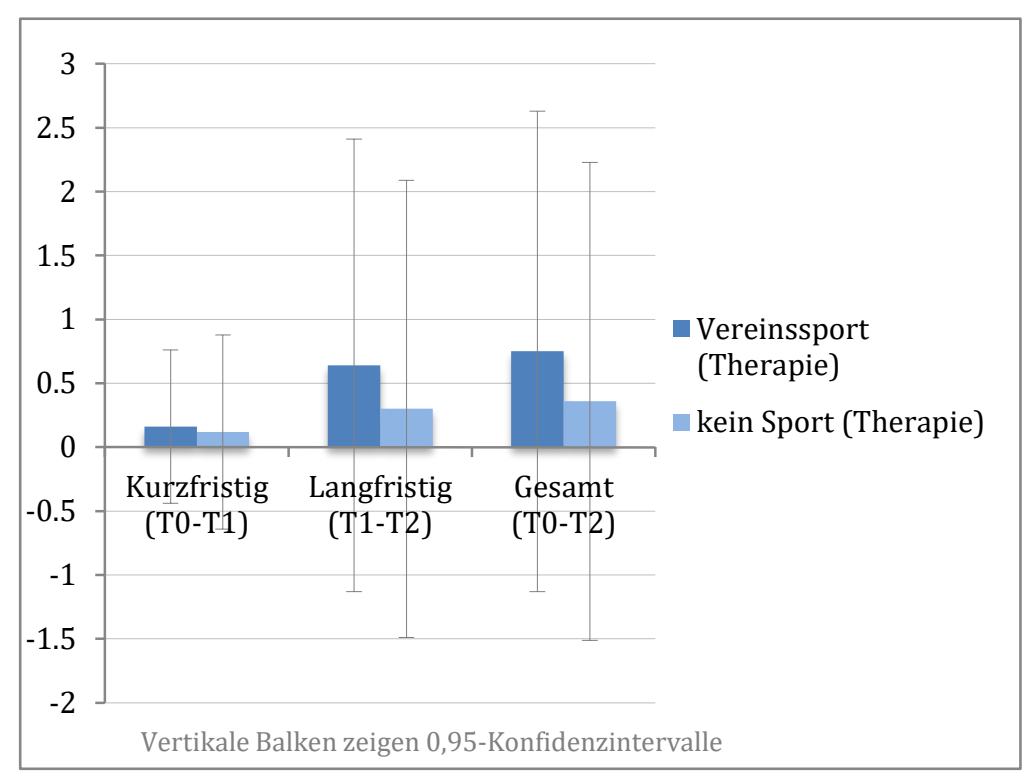

Abbildung 41: Durchschnittliche kurzfristige, langfristige und gesamte BMI-SDS-Reduktion von Patienten mit sportlicher Betätigung im Sportverein sowie von Patienten ohne sportliche Betätigung

Schließlich erfolgte auch an dieser Stelle eine Untersuchung auf Zusammenhänge zu anderen Parametern mit Assoziation zur BMI-SDS-Reduktion (Tabelle 33). Eine Mitgliedschaft im Sportverein war mit einer stärkeren Steigerung der sportlichen Aktivität sowie einem geringeren Alter bei Therapiebeginn assoziiert.

Tabelle 33: Signifikante Zusammenhänge zwischen der Angabe einer Mitgliedschaft im Sportverein und weiteren Parametern mit signifikantem Einfluss auf die BMI-SDS-Reduktion mit Darstellung der entsprechenden Durchschnittssowie $p$-Werte.

\begin{tabular}{|c|c|c|c|}
\hline Parameter & $\begin{array}{l}\text { Mitgliedschaft } \\
\text { Sportverein }\end{array}$ & $\begin{array}{c}\text { Keine } \\
\text { Mitgliedschaft } \\
\text { Sportverein }\end{array}$ & $\mathbf{p}$ \\
\hline & \multicolumn{2}{|c|}{ Durchschnittswert (KI) } & \\
\hline Steigerung körperliche Aktivität & „Häufiger“ & „Gleich“ & 0,04 \\
\hline Steigerung Aktivität im Sportverein & „Häufiger" & „Gleich“ & $<0,0001$ \\
\hline Alter bei Therapiebeginn & $13,13( \pm 0,40)$ & $13,79( \pm 0,44)$ & 0,03 \\
\hline
\end{tabular}

Es bestand kein signifikanter Zusammenhang zu den Parametern „Therapiekonzept“, „Zufriedenheit mit ärztlicher Betreuung“, „Zufriedenheit mit Ernährungsberatung“, „Profit durch 
Ernährungsberatung“, „BMI-SDS bei Therapiebeginn“, „Vorhandensein eines konkreten Zielgewichts vor Therapiebeginn“, „Geschlecht“, „Vorliegen einer Insulinresistenz“, „Erhalt zusätzlicher kinder- und jugendpsychiatrischer Therapie“, „Abgeschlossene Berufsausbildung“ sowie „Mitgliedschaft im Sportverein“ erhoben werden.

\subsubsection{Regelmäßiges Wiegen}

Es gaben 57 (44,5 \%) der zum Follow-up-Zeitpunkt befragten Patienten an, sich regelmäßig zu wiegen. Von diesen gaben 6 ein tägliches (4,7\% der gesamten 128 Patienten), 38 ein wöchentliches (29,7 \%) und 10 ein monatliches (7,8 \%) Intervall an; 3 Patienten machten keine Angaben bezüglich des Wiegeintervalls. Insgesamt führten 66 Patienten $(51,6 \%)$ an, sich nicht regelmäßig zu wiegen, 5 Patienten (3,9\%) machten diesbezüglich keine Angaben.

Es ließ sich über keinen der drei Zeiträume ein Zusammenhang zwischen der Höhe der erreichten Gewichtsreduktion und den Wiegegewohnheiten der Patienten darstellen. 


\section{Diskussion}

\subsection{Zusammenfassung der Hauptergebnisse}

Ziel der vorliegenden Arbeit ist die Erfassung möglicher Einflussfaktoren auf den langfristigen Therapieerfolg einer Adipositastherapie im Kindes- und Jugendalter. Die Zahl der Arbeiten zum Thema der Prädiktoren für erfolgreiche Interventionen ist momentan noch unzureichend. Die Erfassung solcher Faktoren ist jedoch insofern von praktischer Bedeutung, als das Wissen um Prädiktoren für den Therapieerfolg eine „maßgeschneiderte“ Therapie ermöglichen könnte. So kann, anstatt jedem übergewichtigen und adipösen Kind die gleiche Therapie zukommen zu lassen, ein möglichst individueller Ansatz gewählt werden. Auf diese Weise könnten individuelle BMI-SDS-Reduktionen und Therapieerfolge maximiert werden.

Zu diesem Zweck wurden 358 Kinder und Jugendliche, die im Zeitraum von 2004 bis 2012 eine Adipositastherapie im SPZ der Universitätsmedizin Göttingen erhalten hatten, in eine Kohortenstudie eingeschlossen. Für 149 dieser Patienten wurden auch zum Follow-upZeitpunkt Daten erfasst. Es wurde für verschiedene Parameter ein Zusammenhang zwischen der kurzfristigen BMI-SDS-Reduktion dieser Patienten über den Therapiezeitraum sowie der langfristigen, postinterventionellen Reduktion sowie der Reduktion über den gesamten Verlauf ermittelt. Die durchschnittlich erreichte BMI-SDS-Reduktion nach pP-Analyse lag kurzfristig bei 0,07, langfristig bei 0,47 und über den Gesamtverlauf bei 0,56 (nach ITT: kurzfristig: 0,07; langfristig 0,17; gesamt: 0,21). Dabei ging ein größerer Erfolg in der Gewichtsabnahme über den Therapiezeitraum auch mit niedrigeren BMI-SDS-Werten zum Follow-up-Zeitpunkt einher. Ein Anteil von 50,78 \% der Patienten gab weiterhin eine aktivere Freizeitgestaltung durch die Therapie an.

Verschiedene Parameter waren für mindestens einen der drei Zeiträume signifikant mit der BMI-SDS-Reduktion assoziiert. Hierzu zählen:

Parameter mit Assoziation zu einer höheren BMI-SDS-Reduktion:

Therapiebezogen:

- Höhere BMI-SDS-Reduktion über die Therapie (Assoziation mit besseren Gesamterfolgen)

- Steigerung der körperlichen Aktivität (generell sowie im Sportverein)

- Bessere Bewertungen von ärztlicher Betreuung und Ernährungsberatung

Therapieunabhängig:

- Konkretes Ziel (kg) in der Gewichtsreduktion vor Therapiebeginn

- Niedrigeres (kurzfristig) bzw. höheres (langfristig, Gesamtverlauf) Alter bei Therapiebeginn

- $\quad$ Niedrigerer BMI-SDS bei Therapiebeginn

- Männliches Geschlecht

Sonstige:

- Mitgliedschaft im Sportverein

Parameter mit Assoziation zu einer niedrigeren BMI-SDS-Reduktion:

Therapieunabhängig:

- Vorliegen einer Insulinresistenz

- Therapieunabhängige kinder- und jugendpsychiatrische Behandlung

- Fehlende Berufsausbildung

Für folgende Parameter konnte kein signifikanter Zusammenhang zur BMI-SDS-Reduktion erhoben werden:

- Therapiekonzept 
- Entwicklung der Freizeitaktivitäten „Medienkonsum“, „Aktivitäten mit Freunden“ und „Familienaktivitäten“

- Therapiedauer

- Bewertung der psychologischen Betreuung

- Therapiemotivation

- Kindlicher Schulabschluss

- Elterlicher Schulabschluss und elterliche Berufssituation

- Familien- und Wohnsituation

- Migrationsintergrund

- Elterlicher Gewichtsstatus

- Sportliche Aktivität außerhalb eines Sportvereins

- Regelmäßiges Wiegen

Die Aussagekraft dieser Ergebnisse bezüglich der verschiedenen Parameter wird im Kapitel „Diskussion der Ergebnisse“ erörtert.

\subsection{Methodik}

\subsubsection{Patientenkollektiv und Studiendesign}

Eingeschlossen wurden alle Kinder und Jugendlichen, die vom 01.10.2004 bis zum 30.09.2012 eine Adipositastherapie im SPZ des Universitätsklinikums Göttingen erhalten hatten und bei Therapiebeginn mindestens elf Jahre alt waren. Bis zum festgesetzten zeitlichen Endpunkt erfolgte also eine uneingeschränkte Eingliederung aller Patienten, die diese Kriterien erfüllten. Dies ermöglichte die Erfassung einer relativ großen und für die Therapie möglichst repräsentativen Kohorte. Dieses Studiendesign ließ sich allerdings nicht mit der Option einer Kontrollgruppe vereinbaren, sodass keine Vergleichsmöglichkeit für die ermittelten Ergebnisse vorliegt. Insgesamt ähnelt der nicht-kontrollierte Aufbau dem verschiedener anderer Studien zum Thema der Langzeiterfolge von Therapieangeboten (Böhler et al. 2012; Wiegand et al. 2014). Dennoch wäre das Hinzufügen einer Kontrollgruppe, wie es in einigen anderen Arbeiten vorgenommen wurde, wünschenswert gewesen (Mühlig et al. 2014; Oude Luttikhuis et al. 2009). Weiterhin ermöglicht ein prospektiver Aufbau, wie er etwa für die EvAKuJ-Studie gewählt wurde, eine vergleichsweise einheitlichere Datenerhebung (Böhler et al. 2012). Zur Sicherung der erhobenen Ergebnisse wären daher zukünftige weitere Untersuchungen in einem prospektiv und kontrolliert angelegten Setting sinnvoll.

Im Vergleich mit anderen Studien, die sich mit dem Alter bei Therapiebeginn als Prädiktor für den Therapieerfolg beschäftigen, sind die Patienten der vorliegenden Studie älter. Grund ist die Begrenzung des Kollektivs auf mindestens 11 Jahre mit Einschluss von Patienten im Alter von 11 bis 17,95 Jahren. In der APV-Datenbankanalyse von Wiegand et al. wurde eine breitere Altersspanne von 5 bis 25 Jahren bei Therapiebeginn gewählt (Wiegand et al. 2014). Reinehr et al. schlossen Patienten im Alter von 4 bis 16, Braet et al. Patienten von 7 bis 17 Jahren und die EvAKuJ-Studie Altersgruppen von acht bis 16,9 in ihre Studien ein (Böhler et al. 2012; Braet 2006; Reinehr et al. 2010). Diese breiteren Spannen sind auch mit einem größeren Informationsgewinn bezüglich der Entwicklung jüngerer Kinder verbunden. Dennoch waren es in vorangegangenen Studien gerade die älteren Kinder, die häufig nur geringere Erfolge in der Gewichtsreduktion erzielten (Reinehr et al. 2010; Wiegand et al. 2014). Gerade hier ist also eine Erfassung von Prädiktoren mit dem Ziel einer zukünftigen Verbesserung von Langzeiterfolgen relevant. Zusätzlich wurde für diese Altersklassen durch den Nachbeobachtungszeitraum von durchschnittlich vier Jahren für einen großen Teil der Kinder eine Untersuchung der Entwicklung über die Pubertät hinaus und bis ins Erwachsenenalter hinein ermöglicht.

\subsubsection{Datenerhebung}

Die Datenerhebung zu Beginn und Ende der Therapie erfolgte aus den Patientenakten. Den Informationen für Körpergewicht- und -größe lagen die im SPZ Göttingen üblichen Messbedingungen zu Grunde, dies beinhaltet eine Körpergrößenbestimmung mithilfe eines 
Messstabs sowie das Wiegen der Kinder bzw. Jugendlichen in Unterwäsche. Es erfolgte demnach für die Zeitpunkte $\mathrm{T}_{0}$ und $\mathrm{T}_{1}$ eine einheitliche Datenerhebung, was eine gute Vergleichbarkeit der beiden Datensätze ermöglicht. Grundsätzlich wäre dem gewählten Konzept jedoch eine standardisierte und prospektive Erhebung, wie sie etwa in der EvAKuJ-Studie vorgenommen wurde, vorzuziehen (Böhler et al. 2012).

Die Erhebung des Gewichtsstatus zum Follow-up-Zeitpunkt erfolgte dagegen hauptsächlich (zu 78,5\%) aus Selbstangaben der ehemaligen Patienten/-innen. Die Angaben erfolgten durch Ausfüllung bzw. telefonisches Abfragen des zugesandten Fragebogens. Für die übrigen 21,5 \% erfolgte eine Erfassung aktuellerer Gewichts- und Größendaten aus dem krankenhausinternen Patienteninformationsprogramm. Selbstangaben von Gewichts- und Größendaten sind allgemein mit einer geringen Fehlerquote behaftet (Galan et al. 2001). Gerade in den höheren BMI-Klassen kann es jedoch zu einer Unter-, in den niedrigeren BMI-Klassen zu einer geringen Überschätzung kommen (Galan et al. 2001; Sherry et al. 2007). Da der BMI-Status der Studienteilnehmer jedoch erwartungsgemäß eher in den oberen BMI-Kategorien einzuordnen ist, ist eine gewisse Verzerrung der Ergebnisse anzunehmen. Insgesamt konnten die meisten signifikanten Ergebnisse - vermutlich nicht zuletzt aufgrund der beinahe doppelt so großen Datenzahl von Zeitpunkt $\mathrm{T}_{1}$ im Vergleich zu $\mathrm{T}_{2}$ - für die Gewichtsentwicklung über den (repräsentativeren) Therapiezeitraum erhoben werden. Mithilfe der Follow-up-Daten ist eine Nachverfolgung dieser Zusammenhänge möglich. Die Daten zum Follow-up-Zeitpunkt ermöglichen also, selbst wenn keine Objektivität der Angaben vorausgesetzt wird, eine Darstellung von Trends in der langfristigen Gewichtsentwicklung.

Auch in einige andere Studien gehen Selbstangaben für Gewicht und Größe mit ein, hierzu zählen die Referenzwerte für die BMI-Perzentile von Kindern und Jugendlichen nach KromeyerHauschild und die KiGGS-Welle 1 (Brettschneider et al. 2015; Kromeyer-Hauschild et al. 2001). Prinzipiell ist dem jedoch auch an dieser Stelle eine standardisierte Messung zu allen Untersuchungszeitpunkten vorzuziehen, um repräsentative und vergleichbare Messwerte zu erzielen (RKI 2013).

Neben dem Gewicht basieren auch weitere Informationen, wie etwa Angaben zum Freizeitverhalten der Patienten, auf Selbstangaben. Damit ist auch in diesem Fall aufgrund der Subjektivität der Angaben mit einer Verzerrung zu rechnen (Pettee Gabriel et al. 2012). Es muss von einer Anpassung der Angaben an die soziale Erwünschtheit mit Überschätzung der eigenen sportlichen Betätigung ausgegangen werden (Kahlert und Brand 2011).

Daneben ist auch die retrospektive Erfassung von Therapiemotivation und -ziel sowie Zufriedenheit mit und Profit von den Therapiemodalitäten kritisch zu betrachten. So kann man vermuten, dass die Angaben zu Therapiemotivation und -ziel durch die Therapieerfahrungen beeinflusst worden sind. Auch ist anzunehmen, dass sich die Bewertung der einzelnen Modalitäten durch die Patienten an der erreichten Gewichtsreduktion orientiert. Prinzipiell wäre eine Abfrage vor Behandlungsbeginn aussagekräftiger, wie sie auch im Rahmen verschiedener Therapiekonzepte vorgenommen wird (Dobe et al. 2011; Ernst und Wiegand 2010; Petersen und Schlesinger 2009).

\subsubsection{Methoden und Parameter der Datenanalyse}

Der Gewichtsvergleich für die einzelnen Patienten erfolgte mithilfe der Errechnung der BMISDS-Reduktionen über die verschiedenen Zeiträume. Diese Methode ermöglicht eine sensible Darstellung individueller Gewichtsveränderungen, insbesondere auch in Bereichen oberhalb der 99,9. Perzentile (Kromeyer-Hauschild et al. 2001). Aus diesem Grund findet sie in Studien mit ähnlicher Thematik regelmäßig Anwendung (Böhler et al. 2012; Mühlig et al. 2014; Reinehr et al. 2007; Wiegand et al. 2014). Mithilfe verschiedener statistischer Tests wurde überprüft, ob ein Zusammenhang zur BMI-SDS-Reduktion über die Therapiezeit, die postinterventionelle Zeit oder den gesamten Verlauf bestand. Eine ähnliche Vorgehensweise findet sich auch in der EvAKuJ-Studie (hier wurden neben der Reduktion bei Therapieende allerdings zwei Follow-upZeitpunkte ein und zwei Jahre nach Therapieende gewählt) (Böhler et al. 2012). Der Vorteil dieser separaten Betrachtung der verschiedenen Zeiträume liegt in der Möglichkeit einer 
Differenzierung von kurzfristigen und langfristigen Erfolgen. Ebenso kann zwischen Prädiktoren für den kurzfristigen und Prädiktoren für den langfristigen Therapieerfolg unterschieden werden. Eine ausführliche Diskussion der erfassten Parameter findet sich im nachfolgenden Kapitel.

\subsection{Diskussion der Ergebnisse}

\subsubsection{Patientenkollektiv}

Es wurden 358 den Kriterien entsprechende Patienten in die Studie eingegliedert. Vergleicht man diese Zahl mit dem Kollektiv der APV-Datenbank-Auswertung von Wiegand et al. mit 29.181 untersuchten Patienten, ist sie gleichwohl statistisch wenig repräsentativ. Dennoch konnten signifikante Ergebnisse erhoben werden und im Vergleich zu den meisten anderen Arbeiten ähnlicher Thematik ist die Zahl der rekrutierten Patienten hoch. So umfasste eine Studie von Braet et al. beispielsweise 122 (Braet 2006), eine Analyse von Siegfried et al. 85 (Siegfried et al. 2006) und eine Untersuchung von Reinehr et al. ein Kollektiv von 75 Patienten (Reinehr et al. 2003). Damit kann die relativ hohe Patientenzahl nichtsdestotrotz als verhältnismäßige Stärke der vorliegenden Studie betrachtet werden.

Die Loss-to-Follow-up-Rate der vorliegenden Arbeit lag bei 64,2\%. Dieser Wert stellt sich folglich recht hoch dar. Dies ist für die statistische Auswertung insofern problematisch, als große Datenmengen für die Auswertung verloren gehen und damit die erhobenen Ergebnisse an Repräsentativität verlieren. Zusätzlich besteht insbesondere für das gewählte Studiendesign die Gefahr eines Bias für die Follow-up-Daten-Erhebung. Grund hierfür ist, dass neben fehlender Erreichbarkeit von Patienten, beispielsweise aufgrund von geänderten Kontaktdaten, auch eine Ablehnung zur Teilnahme an der Studie zu einem Loss-to-Follow-up führte. Dies war für 20,9 \% der Patienten der Fall. Es ist jedoch nicht auszuschließen, dass es gerade diejenigen Patienten mit geringeren Therapieerfolgen und schlechteren Erfahrungen mit der Therapie waren, die eine Teilnahme ablehnten. Diese Möglichkeit ist insbesondere insofern zu berücksichtigen, als die durchschnittliche kurzfristige BMI-SDS-Reduktion von 0,07 sich im Vergleich zur langfristigen Reduktion von 0,47 recht gering darstellt. Dieser Unterschied ist möglicherweise auf eine Kombination aus den Effekten einer Selektion zum Follow-up-Zeitpunkt wie auch der BMI-Unterschätzung im Rahmen der Selbstangabe zurückzuführen.

Vergleicht man diese Werte mit den Daten der in der Einleitung vorgestellten Studien, so liegt die Loss-to-Follow-up-Rate von 58,4 \% etwa im Durchschnitt. Die höchsten Loss-to-Follow-upAngaben finden sich dabei in der APV-Analyse nach Wiegand et al. mit $89 \%$. Darauf folgen Mühlig et al. mit $71 \%$ sowie die EvAKuJ-Studie mit 32,1 \% bis 55,2 \%.

Hohe Loss-to-Follow-up-Raten scheinen ein generelles Problem dieser Patientengruppe zur Beurteilung von Langzeitverläufen in der Adipositastherapie darzustellen.

\subsubsection{Therapieerfolg}

Zur Beurteilung des Therapieerfolgs wurden die erreichte BMI-SDS-Reduktion sowie die Entwicklung der Freizeitgestaltung der Patienten beurteilt. Für ersteren Punkt wurde in der vorliegenden Arbeit nach ITT-Analyse kurzfristig eine BMI-SDS-Reduktion von 0,07, langfristig von 0,17 und insgesamt von 0,21 erfasst. Diese Werte liegen im Rahmen des in der Metaanalyse von Mühlig et al. erhobenen Reduktionsbereichs mit Werten zwischen 0,05 bis 0,42 innerhalb von 12 bis 24 Monaten nach Behandlungsbeginn in den untersuchten Studien (Mühlig et al. 2014). Für die acht untersuchten Studien mit den höchsten qualitativen Standards (u.a. einer Analyse nach dem ITT-Prinzip) wurde nach einem Jahr eine Reduktion von 0,05 bis 0,39; nach zwei Jahren eine Reduktion zwischen 0,08 und 0,42 verzeichnet. Diese auf kontrollierten, randomisierten Studien basierenden Daten lassen sich aufgrund ihrer Repräsentativität gut als Vergleich heranziehen. Die Ergebnisse nach einem Jahr sind dabei in zeitlicher Hinsicht annähernd vergleichbar mit denen zum Therapieende (BMI-SDS-Reduktion 0,07), da in der vorliegenden Studie die Therapie im Durchschnitt 12,1 Monate dauerte. Einschränkend muss 
jedoch hinzugefügt werden, dass in den von Mühlig et al. untersuchten Studien die Therapie zu diesem Zeitpunkt in der Regel schon abgeschlossen war.

Beim Vergleich der Ergebnisse liegt die erreichte Reduktion kurzfristig im unteren Bereich der Spanne, während die langfristigen und gesamten Erfolgsquoten etwa im Mittelfeld der Ergebnisse angesiedelt sind. Insgesamt lässt sich dennoch feststellen, dass wie auch in den von Mühlig et al. untersuchten Studien eine Gewichtsnormalisierung im Therapieverlauf nur dann möglich scheint, wenn das Ausgangsgewicht der Patienten bereits nur knapp über der Grenze zum Normalgewicht lag.

Im Hinblick auf die Erfolgsdefinition der AGA konnten nach pP-Analyse in der vorliegenden Studie kurzfristig 21,5\%, langfristig $63 \%$ und über den Gesamtverlauf 68,5 \% der Patienten eine gute Reduktion um mindestens 0,2 erzielen. Auch Wiegand et al. nahmen eine solche Einteilung vor, wobei 2 Jahre nach Therapiebeginn noch 47,1\% der Patienten (ebenfalls nach pP-Analyse) eine BMI-SDS-Reduktion von mindestens 0,2 im Vergleich zum Ausgangsgewicht vorweisen konnten (Wiegand et al. 2014).

Im Vergleich mit dem Gesamtverlauf der vorliegenden Studie erreichten die von Wiegand et al. untersuchten Patienten insgesamt also geringere Reduktionen (siehe Abbildung 42). Die Vergleichbarkeit der Werte ist jedoch auch an dieser Stelle eingeschränkt, da die Erhebung in der vorliegenden Studie im Durchschnitt zu einem wesentlich späteren Zeitpunkt erfolgte. Zudem ist ein Vergleich der Werte bei Therapieende nicht möglich.

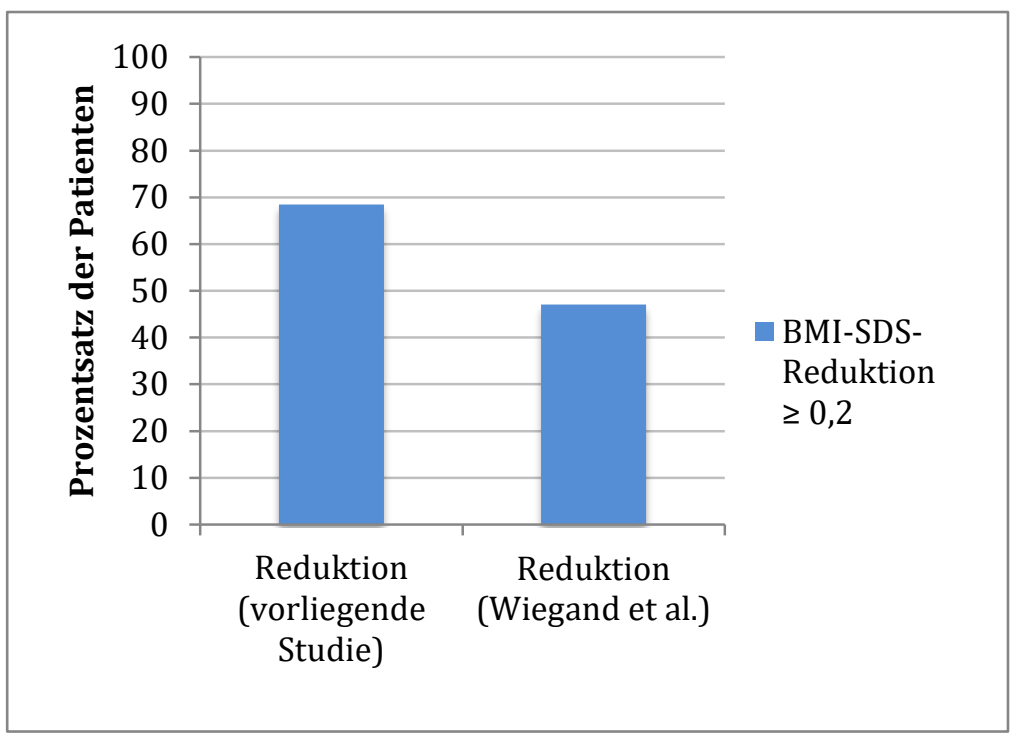

Abbildung 42: Vergleichende Darstellung des Prozentsatzes an Patienten mit Erreichen einer BMI-SDS-Reduktion von $\geq$ 0,2 in der vorliegenden Studie (über den Gesamtzeitraum: $T_{0}-T_{2}$ ) sowie in der APV-Datenbank-Analyse nach Wiegand et al. (jeweils nach pP-Analyse)

In diesem Aspekt besteht eine bessere Vergleichbarkeit mit den Ergebnissen der EvAKuJ-Studie: Nach ITT-Analyse konnten in der vorliegenden Studie kurzfristig 21,5\%, langfristig 26,3 \% und über den Gesamtverlauf 28,5 \% der Patienten gute Erfolge (entsprechend einer BMI-SDSReduktion von mindestens 0,2) erzielen. Nach Böhler et al. konnten im Rahmen der EvAKuJStudie (ebenfalls nach ITT-Analyse) kurzfristig $56 \%$ der Patienten eine BMI-SDS-Reduktion von mindestens 0,2 \% erreichen. Langfristig stellten sich die Erfolge jedoch deutlich geringer dar, wobei zum Follow-up-Zeitpunkt nach einem Jahr nur noch $13 \%$ und nach zwei Jahren $14 \%$ entsprechende Reduktionen erreichten (Böhler et al. 2012). Damit liegt der Prozentsatz mit Erreichen eines guten Erfolgs über die Therapie in der vorliegenden Studie deutlich unter dem der EvAKuJ-Studie. Die Langzeitergebnisse stellen sich dagegen erfolgreicher dar: Der in der EvAKuJ-Studie zu beobachtende Trend einer erneuten Gewichtszunahme nach Beendigung der Therapie ließ sich anhand dieser Werte für die vorliegende Studie nicht darstellen, im Gegenteil erhöhte sich sogar der Prozentsatz mit guten BMI-SDS-Reduktionen. Schließt man allerdings die Patienten, die überhaupt eine Gewichtsreduktion erreichen konnten, in die Betrachtung mit ein, so verringern sich auch in der vorliegenden Studie langfristig die Effektstärken: Während nach 
ITT-Analyse kurzfristig noch 55,9 \% der Patienten ihren BMI-SDS reduzieren konnten, waren es langfristig 28,8 \%, über den Gesamtverlauf $31 \%$. Damit wäre es auch in der vorliegenden Studie einem Großteil der Patienten nicht möglich, auf lange Sicht eine Gewichtsreduktion zu erreichen bzw. aufrecht zu erhalten.

Auch nach pP-Analyse ließ sich dieser Trend in der EvAKuJ-Studie darstellen: Nach Böhler et al. erreichten kurzfristig $60 \%$, mittelfristig (nach einem Jahr) $45 \%$ und langfristig (nach 2 Jahren) $50 \%$ der Patienten eine BMI-SDS-Reduktion um mindestens 0,2 (Böhler et al. 2012). In der vorliegenden Studie erreichten nach pP-Analyse kurzfristig 21,5\%, langfristig $63 \%$ und insgesamt 68,5 \% der Patienten gute Erfolge. Auch an dieser Stelle liegen also die kurzfristigen Ergebnisse unter, die langfristigen über denen der EvAKuJ-Studie. Auch bei Betrachtung der Patienten, die überhaupt eine Reduktion erreichten, übersteigen nach pP-Analyse die langfristigen und gesamten Prozentsätze den kurzfristigen: Während kurzfristig 55,9\% der Patienten ihren BMI-SDS reduzieren konnten, waren es langfristig 69,1 \% und über den Gesamtverlauf sogar 74,5 \%. Nach dieser Betrachtung würde die Adipositastherapie folglich auch auf lange Sicht bei einem Großteil der Patienten zu zufriedenstellenden Erfolgen führen.

Sowohl die ITT- als auch die pP-Analyse sind jedoch kritisch zu betrachten: Im Rahmen der ITTAnalyse ist durch Wertung eines Loss-to-Follow-up als Misserfolg eine Unterschätzung des tatsächlichen Erfolgs zu erwarten. Die pP-Analyse geht dagegen durch das selektierte Patientengut am ehesten mit einer Überschätzung der Ergebnisse einher.

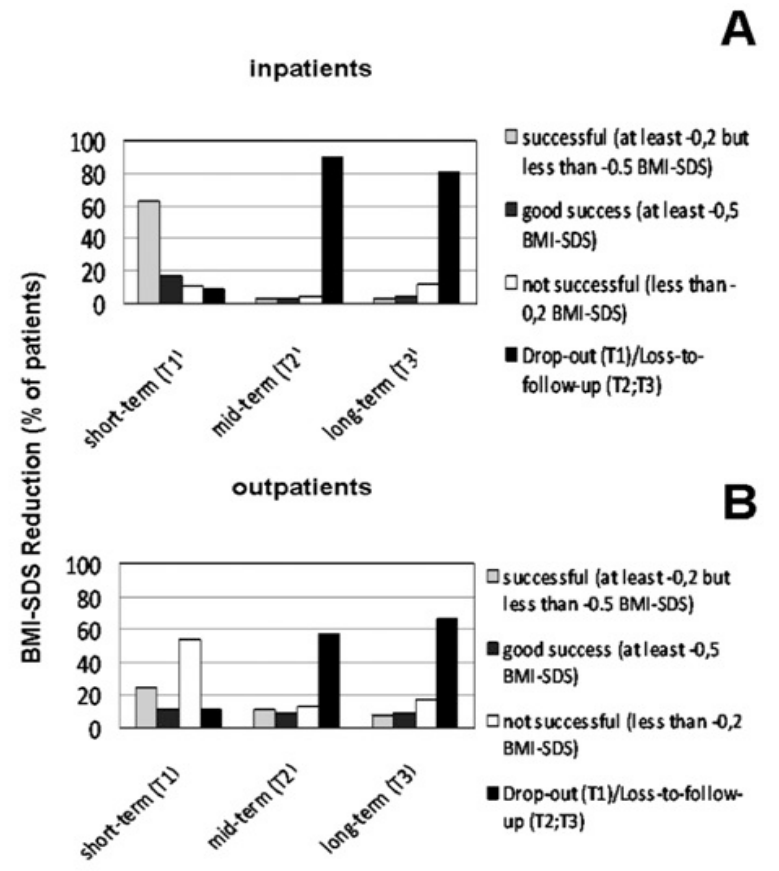

Abbildung 43: Kurzfristige, mittelfristige und langfristige BMISDS-Reduktion im Rahmen der EvAKuJ-Studie mit Darstellung des Prozentsatzes an Patienten mit einer BMI-SDSReduktion von $\geq 0,2, \geq 0,5,<0,2$ sowie der Drop-Out-Rate für ambulante und stationäre Patienten nach Böhler et al. (Böhler et al. 2013)

\section{A}

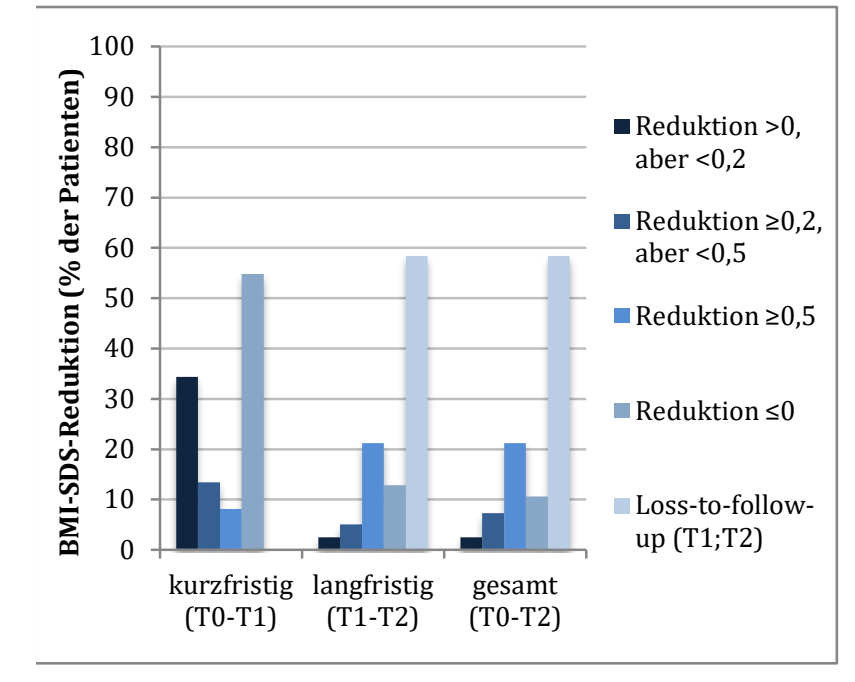

Abbildung 44: Kurzfristiger, langfristiger und gesamter Prozentsatz an Patienten mit BMI-SDS-Reduktion $\leq 0$ (kein Erfolg); >0, aber <0,2 (Gewichtsreduktion); $\geq 0,2$, aber $<0,5$ (guter Erfolg); $\geq 0,5$ (sehr guter Erfolg) nach ITT-Analyse

Neben der BMI-SDS-Entwicklung erfolgte auch eine Untersuchung des Freizeitverhaltens im Rahmen der Therapie. Zum einen wurde hier die körperliche Aktivität betrachtet. Insgesamt gaben 50,78 \% der Patienten eine Steigerung ihrer körperlichen Aktivität durch die Therapie an, $14 \%$ steigerten ihre Aktivität im Sportverein. In 8,59 \% der Fälle wurde eine Verringerung der körperlichen Aktivität bzw. der Aktivität im Sportverein angegeben. Die Entwicklung der körperlichen Aktivität wurde auch im Rahmen der EvAKuJ-Studie untersucht (Böhler et al. 
2012). Es erfolgte eine Erfassung der Tage mit mindestens einer Stunde körperlicher Anstrengung innerhalb einer Woche. Kurzfristig wurde nach pP-Analyse eine Aktivitätssteigerung bei 59,5 \% der Patienten, also um etwa zehn Prozent höher als in der vorliegenden Studie, festgestellt. Allerdings lag die Zahl der Patienten mit Verringerung ihrer körperlichen Aktivität mit 21,8 \% deutlich über den von uns erhobenen Ergebnissen. Diese Ergebnisse sind bei unterschiedlicher Fragestellung jedoch nicht gänzlich vergleichbar.

Im Rahmen des Freizeitverhaltens wurde auch die Entwicklung des Medienkonsums erfasst. 18,8 \% gaben eine Verringerung, 5,5 \% eine Steigerung für diesen Parameter an. In der EvAKuJStudie erfolgte eine weitere Unterteilung in Fernseh- und Videokonsum sowie Computernutzung. Für ersteres kam es nach pP-Analyse für 20,2 \% der Patienten kurzfristig zu einer Verringerung, für 37,3 \% zu einer Steigerung. Bezüglich der Computernutzung kam es bei $14 \%$ der Patienten zu einer Verringerung, während 44,4\% ihre Computernutzung noch steigerten.

Damit konnte im Vergleich in der EvAKuJ-Studie zwar für einen größeren Patientenanteil eine Verringerung des Medienkonsums festgestellt werden, allerdings überstieg dort, im Gegensatz zur vorliegenden Studie, der Prozentsatz der Steigerung den der Verringerung. Unter Zusammenschau der Ergebnisse für Zunahme und Reduktion des Medienkonsums konnten also in der vorliegenden Studie insgesamt höhere Effektstärken erfasst werden.

Es muss jedoch hinzugefügt werden, dass die EvAKuJ-Studie, wie bereits im Kapitel Datenerhebung angesprochen, durch den prospektiven Aufbau mit mehrfachen Datenerhebung zu den verschiedenen Zeitpunkten eine zuverlässigere Erhebungsmethode vornimmt. Dies lässt im Vergleich zu einer retrospektiven Befragung eine bessere Beurteilung zu.

Neben Bewegungsverhalten und Medienkonsum wurde weiterhin auf eine Veränderung der Parameter „Treffen mit Freunden“ und „Familienaktivitäten“ untersucht. Für ersteres gaben $10,1 \%$ eine Steigerung, keiner der Patienten eine Verringerung an. $7 \%$ steigerten laut Selbstangabe ihre Familienaktivitäten, während es bei 0,8 \% zu einer Verringerung kam. Es sind uns momentan keine weiteren Studien bekannt, die aktiv eine Steigerung der mit Freunden bzw. Familie verbrachten Zeit im Rahmen einer Adipositastherapie im Kindes- und Jugendalter untersucht haben. Nach Eberding et al. ist die Anzahl gemeinsamer Aktivität innerhalb von Familien mit adipösen Kindern jedoch begrenzt (Eberding und Lemme 2006). Außerdem weisen diese Familien nach Bosch et al. vergleichsweise dysfunktionale Verhältnisse mit einem relativ geringen emotionalen Zusammenhalt auf (Bosch et al. 2004). Weiterhin kamen verschiedene Untersuchungen $\mathrm{zu}$ dem Ergebnis, dass adipöse Kinder innerhalb ihrer Altersgruppen mit erheblichen Vorurteilen belastet sind, was die soziale Integration erschweren kann (Hansson et al. 2009; Hill und Silver 1995).

Damit ist - auch ohne Vergleichsmöglichkeiten mit den Verläufen anderer Studien - eine Steigerung von mit Familien und Freunden unternommenen Aktivitäten als positiv zu bewerten.

Insgesamt ließen sich laut Patientenangaben sowohl Bewegungsverhalten als auch Medienkonsum sowie Aktivitäten mit Freunden und Familie im Rahmen der Therapie steigern. Insbesondere die Zunahme des Bewegungsverhaltens bei 48,4\% der Patienten ist dabei erfreulich, während die positiven Veränderungen in Bezug auf die anderen Parameter geringer ausfielen.

Die Zusammenhänge zwischen der Entwicklung der Freizeitgestaltung und der Entwicklung der BMI-SDS werden im nächsten Kapitel ausführlich beleuchtet.

\subsubsection{Untersuchung des Einflusses therapiebezogener Parameter auf die BMI- SDS-Reduktion}

\subsubsection{Therapiekonzept}

Betrachtet man das Therapiekonzept, so zeigten sich kurzfristig die 8,3\% der Patienten, die eine multiprofessionelle Gruppentherapie erhalten hatten, am erfolgreichsten. Die erreichte 
durchschnittliche BMI-SDS-Reduktion lag bei 0,1. Langfristig erreichten dagegen die Patienten mit ausschließlich ärztlichen Terminen (4,7 \%) mit einer BMI-SDS-Reduktion von 0,8 die größten Erfolge. Über den Gesamtverlauf erzielten erneut Patienten mit multiprofessioneller Behandlung in der Gruppe (7,8 \%) mit durchschnittlich 0,7 die höchste BMI-SDS-Reduktion. Dennoch sind diese Ergebnisse nicht signifikant und insbesondere durch die niedrigen Patientenzahlen mit ausschließlich ärztlichen Terminen wenig repräsentativ. Weiterhin stehen die Ergebnisse in Bezug auf eine rein ärztliche Behandlung nicht im Einklang mit den Ergebnissen bisheriger Untersuchungen. So haben sich laut den Leitlinien der AGA mit Bezug auf eine umfangreiche Studienlage die Anwendung einer Ernährungsschulung, Verhaltenstherapie, körperliche Aktivität sowie die Einbindung einer Bezugsperson in der kindlichen Adipositastherapie bewährt (Wabitsch und Kunze 2015). Insbesondere ist auch eine Kombination der verschiedenen Bausteine mit einem größerem Therapieerfolg assoziiert (Caroli und Burniat 2006; Parizkova et al. 2002). Ärztliche Termine sind folglich für die Diagnostik, Therapie und Aufklärung über mögliche Ursachen bzw. Folgen der Adipositas unerlässlich, höhere BMI-SDS-Reduktionen sind jedoch generell beim Zusatz weiterer Bausteine zu erwarten. Es ist ebenfalls zu erwägen, ob für Patienten mit dem Erhalt ausschließlich ärztlicher Termine von vornherein ein geringer Therapiebedarf bestand und sie auch in diesem Zusammenhang bessere Ergebnisse erzielen konnten.

In Anbetracht dieser Umstände scheint gerade die Betrachtung der kurzfristigen Ergebnisse repräsentativer, da diese auf dem verlässlichsten und größten Datensatz der Studie beruhen. Wie bereits erwähnt, zeigte sich für diesen Zeitraum die multiprofessionelle Gruppentherapie mit Einbau der Bausteine Ernährung, Medizin und psychologische Betreuung am erfolgreichsten (BMI-SDS-Reduktion 0,1). Darauf folgt die multiprofessionelle Einzeltherapie (Medizin, Ernährungsschulung, psychologische Betreuung) mit einer Reduktion von 0,07, an dritter Stelle die individuelle Einzeltherapie (Medizin und Ernährungsberatung / psychologische Betreuung) mit einer Reduktion von 0,06 und erst an letzter Stelle die rein ärztliche Betreuung mit einer SDS-Zunahme um 0,01. Trotz fehlender Signifikanz scheinen diese Ergebnisse im Hinblick auf die aktuelle Studienlage recht realistisch. Zusätzlich ist für diesen Zeitraum eine Vermittlung des Erfolgs in der BMI-SDS-Reduktion über einen anderen signifikanten Parameter unwahrscheinlich. Grund ist, dass bei Betrachtung der Parameter mit signifikanter Assoziation zur BMI-SDS-Reduktion lediglich ein Zusammenhang zum Vorhandensein eines konkreten Zielgewichts vor Therapiebeginn und dem Vorliegen einer Insulinresistenz erfasst wurde. Beide Parameter waren mit einem intensiveren Therapiekonzept assoziiert. Zwar hing das Vorhandensein eines Zielgewichts mit einer signifikant höheren Gewichtsreduktion zusammen, allerdings galt dies nur für den langfristigen und gesamten Zeitraum, sodass kurzfristig keine Einflussnahme anzunehmen ist. Das Vorliegen einer Insulinresistenz war dagegen kurzfristig sogar mit einer geringeren Gewichtsreduktion assoziiert, sodass diese Assoziation ebenfalls die guten Therapieerfolge intensiver Therapiekonzepte nicht erklären kann.

Nach diesen Ergebnissen scheint es prinzipiell also sinnvoll, jedem übergewichtigen und adipösen Kind den Zugang zu einer multiprofessionellen Gruppentherapie zu ermöglichen. Diese ist jedoch im Vergleich zu den anderen Therapiekonzepten kosten- und zeitaufwändiger. So wurden hier die höchsten Zahlen in Bezug auf Terminanzahl $(23,5)$ und Therapiedauer $(1,5$ Jahre) erreicht. Dies würde folglich enorme Kapazitäten in Anspruch nehmen, wobei insgesamt nur eine geringere Zahl übergewichtiger bzw. adipöser Kinder und Jugendlicher überhaupt einer Therapie zugeführt werden könnte. Dementsprechend empfiehlt es sich, die Patienten zunächst in ein Vorprogramm aufzunehmen und ihre Motivation sowie die medizinisch indizierten Therapiedisziplinen für Patient und Eltern zu prüfen. Auf diese Weise können die knappen Ressourcen sinnvoll eingesetzt werden.

\subsubsection{BMI-SDS-Reduktion über die Therapie}

Interessanterweise zeigte sich ein hochsignifikanter Zusammenhang der BMI-SDS-Reduktion über den Therapieverlauf und der BMI-SDS-Reduktion über den Gesamtverlauf. Folglich konnten Patienten mit hohen Reduktionen über den Therapiezeitraum auch insgesamt höhere Reduktionen erreichen. Es bestand ebenfalls eine signifikante Negativkorrelation zwischen dem 
aktuelleren BMI-SDS zu Zeitpunkt $\mathrm{T}_{2}$ und der Gewichtsreduktion über den Therapiezeitraum. Damit sind höhere BMI-SDS Reduktionen über die Therapie auch zum Follow-up-Zeitpunkt noch mit einem niedrigeren BMI-SDS Gewichtsstatus assoziiert. Im Gegensatz dazu ließ sich keine signifikante Korrelation zwischen den Werten der interventionellen und postinterventionellen BMI-SDS Reduktionen ermitteln. Demzufolge waren die Patienten, die langfristig eine Gewichtsreduktion erreichen konnten, nicht regelhaft dieselben Patienten, die über den Therapieverlauf ihr Gewicht reduzieren konnten. Dennoch legt die Korrelation zwischen kurzfristiger Gewichtsreduktion und BMI-SDS zum Follow-up-Zeitpunkt nahe, dass die im Rahmen der Therapie erfolgreichen Patienten im Schnitt das erreichte niedrigere Gewichtsniveau halten und also eine Stabilisierung ihres Gewichts erreichen konnten. Im Rahmen therapeutischer Interventionen sind generell nicht nur kurz- sondern auch langfristige Erfolge anzustreben, um die Entwicklung von Komorbiditäten und eine Übertragung ins Erwachsenenalter zu vermeiden (Lloyd et al. 2010). Sieht man in diesem Zusammenhang auch die Gewichtsstabilisierung der im Rahmen der Therapie erreichten Reduktion als Erfolg an, so ist in der vorliegenden Studie eine erfolgreiche kurzfristige Gewichtsabnahme ebenfalls als Prädiktor für langfristigen Therapieerfolg zu betrachten. In verschiedenen Fällen konnten Faktoren identifiziert werden, die signifikant mit den kurzfristigen, jedoch nicht mehr mit den langfristigen Reduktionen assoziiert waren. Dies ist vermutlich zumindest teilweise auf das um fast $50 \%$ kleinere Kollektiv zum Follow-up-Zeitpunkt zurückzuführen. Grund hierfür ist, dass Unterschiede im Vergleich kleinerer Gruppen zum Erreichen des Signifikanzniveaus eine höhere Ausprägung aufweisen müssen.

Ähnliche Ergebnisse konnten auch in anderen Studie erhoben werden, so etwa in einer Untersuchung der Ergebnisse des Obeldicks-Therapiekonzepts nach Reinehr et al.: Hier war die BMI-SDS-Reduktion ein Jahr nach Therapiebeginn ein starker Prädiktor der langfristigen BMISDS-Reduktion zwei Jahre nach Therapiebeginn (Reinehr et al. 2010).

Auch in der Zehn-Jahres-Follow-up-Studie nach Epstein et al. ließ sich ein ähnlicher Zusammenhang beobachten: In dieser Untersuchung war der beste Prädiktor für die Gewichtsveränderung nach zehn Jahren die Veränderung innerhalb der ersten 5 Jahre.

Aus diesen Zusammenhängen lässt sich schließen, dass Prädiktoren, die mit hohen Gewichtsreduktionen über den Therapieverlauf verknüpft sind, sich auch positiv auf die langfristige Gewichtsentwicklung auswirken können. Der Untersuchung dieser Prädiktoren kommt folglich weiterhin eine große Bedeutung $\mathrm{zu}$, da sie indirekt als Prädiktoren des Therapieerfolgs auf lange Sicht betrachtet werden können. Im Einzelfall sind jedoch spezifische Untersuchungen der langfristigen Auswirkungen der jeweiligen Parameter notwendig.

\subsubsection{Entwicklung des Freizeitverhaltens}

Bezüglich des Adipositas-fördernden Verhaltens wurde zum einen das Sport- und Bewegungsverhalten der Patienten untersucht. Dabei war kurzfristig eine Steigerung der sportlichen Aktivität innerhalb wie auch außerhalb eines Sportvereins signifikant und deutlich mit der Gewichtsreduktion assoziiert. Für eine Aktivität im Sportverein ließ sich auch langfristig und über den Gesamtverlauf noch eine mäßige, wenn auch nicht signifikante Korrelation feststellen. Dagegen stellte sich über diese Zeiträume für eine Steigerung der sportlichen Aktivität außerhalb eines Vereins kein Zusammenhang mehr dar. Auch in Bezug auf eine sportliche Aktivität per se (siehe Kapitel „Sportliche Aktivität“) ließ sich ein größerer Zusammenhang zwischen Gewichtsreduktion und vereinssportlicher Aktivität als einer anderweitigen sportlichen Betätigung feststellen.

Insgesamt gehört eine Steigerung der körperlichen Aktivität, insbesondere bei Ausübung eines Vereinssports über den Therapiezeitraum, zu den wichtigsten Prädiktoren für eine Gewichtsreduktion in der vorliegenden Studie. Dennoch müssen auch diese Ergebnisse in Anbetracht der retrospektiven Erhebung sowie der Selbstangaben mit Vorsicht behandelt werden.

$\mathrm{Zu}$ berücksichtigen ist in diesem Zusammenhang ebenfalls, dass eine Steigerung der körperlichen Aktivität bzw. Aktivität im Sportverein auch mit höheren Profitangaben von der Ernährungsberatung sowie einem jüngeren Alter bei Therapiebeginn einherging. Beide Parameter waren ebenfalls mit einer signifikant höheren BMI-SDS-Reduktion über den 
Therapiezeitraum verknüpft. Es stellt sich die Frage, ob sich tatsächlich alle drei Parameter kausal auf den Erfolg in der Gewichtsentwicklung auswirken oder ob ein Zusammenhang lediglich durch einen der anderen Parameter vermittelt wird. Letzteres ist für die körperliche Aktivität zwar nicht auszuschließen, die positiven Auswirkungen einer Aktivitätssteigerung auf die Gewichtsentwicklung wurden jedoch bereits vielfach nachgewiesen, sodass eine Kausalität zumindest angenommen werden kann (Atlantis et al. 2006; Epstein und Valoski und et al. 1990; Flores 1995). Interessant ist jedoch auch die Frage, welche Art von körperlicher Aktivität sich für die Adipositastherapie am ehesten eignet. So zeigten sich in einer Zehn-Jahres-Follow-upStudie von Epstein et al., dass Patienten, die an unterschiedlichen sportlichen Interventionen teilnahmen, auch unterschiedlich erfolgreich waren (Epstein et al. 1994). Zwei von drei untersuchten Gruppen wurden Sportprogrammen mit einem Energieverbrauch entsprechend einer Gehstrecke von 6,4 Kilometern täglich bzw. dem Verbrauch von 2800 Kalorien wöchentlich (bezogen auf eine Person mit einem Gewicht von 68kg) zugeführt. Die dritte Gruppe wurde mit Informationen $\mathrm{zu}$ verschiedenen Freiübungen ausgestattet. Die Gewichtsreduktion der beiden ersten Gruppen überstieg dabei signifikant die der dritten Gruppe. Eine Stärke dieser Studie ist dabei zum einen das prospektive und kontrolliert angelegte Setting und zum anderen der lange Follow-up-Zeitraum.

Es lassen sich Parallelen zu den Ergebnissen der vorliegenden Arbeit ziehen: In beiden Untersuchungen zeigten sich diejenigen Patienten insgesamt erfolgreicher, deren sportliche Betätigung regelmäßig in einem extern kontrollierten Rahmen stattfand. Es lässt sich vermuten, dass Patienten, die einen Vereinssport ausüben, eine konstantere und womöglich auch intensivere sportliche Aktivität betreiben, als solche, die außerhalb eines Vereins trainieren. Generell sind die meisten Formen der sportlichen Aktivität im Rahmen einer Adipositastherapie zu unterstützen. Dennoch legen die Ergebnisse insgesamt nahe, dass sich ein kontrolliertes und regelmäßiges Interventionssetting noch besser auf die Gewichtsreduktion auswirkt.

Auch bei der Untersuchung des Medienkonsums zeigte sich kurzfristig eine relativ hohe Korrelation zwischen einer Reduktion und einer höheren Gewichtsabnahme. Auf niedrigerem Niveau zeigte sich dies auch für den Gesamtverlauf. Dennoch sind die Ergebnisse statistisch nicht signifikant, ein Zusammenhang kann daher nur vermutet werden.

Ähnliche Ergebnisse erzielten Epstein et al. 1995: Anhand 16 Jugendlicher zwischen 12 und 16 Jahren wurde der Zusammenhang zwischen einer Reduktion sitzender Tätigkeiten wie Fernsehen und das Spielen von Computer- und Videospielen und der Energieaufnahme untersucht. Eine Reduktion der sitzenden Tätigkeit ging auch mit einer signifikanten Reduktion der Energieaufnahme einher. Wünschenswert wäre allerdings die Untersuchung eines direkten Zusammenhangs zwischen sitzender Tätigkeit und Gewicht gewesen. Weiterhin ist die Selbstangabe der Energieaufnahme, wie auch die Angabe bezüglich der Reduktion des Medienkonsums in der vorliegenden Studie, kritisch zu betrachten. Eine Beschränkung des Medienkonsums im Rahmen einer Adipositastherapie im Kindes- und Jugendalter scheint insgesamt dennoch sinnvoll.

Schließlich erfolgte eine Untersuchung der Entwicklung von Aktivitäten mit Freunden und Familie. Es konnte kein Zusammenhang zwischen einer Steigerung der genannten Parameter und der BMI-SDS-Reduktion über die verschiedenen Zeiträume festgestellt werden. Dabei bleibt fraglich, ob der Zusammenhang im Rahmen des statistisch gesehen recht kleinen Kollektivs nicht darstellbar war oder ob tatsächlich kein Zusammenhang vorliegt.

Bei der Untersuchung von Familien mit mindestens einem adipösen Kind fällt auf, dass nur wenige Aktivitäten im Rahmen der Familien unternommen werden (Eberding und Lemme 2006). Wie bereits im Kapitel „Therapieerfolg“ erwähnt sind die Kontakte innerhalb der Familien durch vergleichsweise dysfunktionale Faktoren wie ein Überwiegen negativer Interaktionen und einen geringen emotionalen Zusammenhalt geprägt. Verschiedene Studien konnten jedoch positive Einflüsse der Einbindung des sozialen Umfelds (Barlow 2007; Cousins et al. 1992; Gidding et al. 2006)) auf die Gewichtsentwicklung aufzeigen. Eine Förderung von Familienaktivitäten könnte insofern mit einer Steigerung der familiären Funktionalität und einer Erhöhung der Ressourcen zur Unterstützung der kindlichen Therapie einhergehen. 


\subsubsection{Therapiedauer und postinterventioneller Zeitraum}

Eine Stärke der vorliegenden Studie ist die Länge der untersuchten Zeiträume. Zum einen betrifft dies den Zeitraum der Therapie selbst. Der durchschnittliche Therapiezeitraum umfasste 12,1 Monate, der längste sogar 4,8 Jahre. Diese Zeiträume liegen über den in diversen Studien gewählten Mindest-Therapiedauern. So lag die zur Eingliederung mindeste Therapiezeit in der Metaanalyse von Oude-Luttikhuis et al. bei sechs Monaten, bei Mühlig et al. bei 9,3 Monaten (bezogen auf die acht methodisch besten eingegangenen Arbeiten) und in der EvAKuJ-Studie bei 11,4 Monaten. Es ist jedoch zu bedenken, dass durch die Einschlusskriterien der vorliegenden Arbeit auch Patienten mit nur sehr geringen Therapiezeiträumen in die Auswertung eingingen, insofern steht sie den anderen Untersuchungen in diesem Punkt nach. Da jedoch die Werte der BMI-SDS-Reduktionen gegen die der Therapiezeit korreliert wurden, ermöglichte dies eine Untersuchung des Einflusses sehr unterschiedlicher Interventionsdauern auf die Gewichtsreduktion.

Es zeigte sich für keinen der drei Zeiträume ein signifikanter Zusammenhang zwischen Therapiedauer und BMI-SDS-Reduktionen. Für die kurzfristige und langfristige Reduktion lagen nur geringe Korrelationen bei relativ hohen p-Werten vor. Allerdings konnte für den gesamten Zeitraum eine recht hoher Zusammenhang mit der BMI-SDS-Reduktion festgestellt werden $\left(r^{2}=0,88\right)$. Bei einem p-Wert von 0,29 ist diese Assoziation statistisch zwar nicht gesichert, lässt sich jedoch zumindest vermuten.

Im Rahmen der EvAKuJ-Studie war eine längere Behandlungsdauer ebenfalls mit einem größeren Therapieerfolg assoziiert, dies galt insbesondere für die ambulante Therapie (Böhler et al. 2012). Auch hier fiel die Korrelation insgesamt jedoch relativ gering aus.

Es wird verschiedentlich vermutet, dass eine längere Therapiedauer durch Festigung der erlernten Verhaltensweisen mit größeren Erfolgen einhergeht (Disse und Zimmer 2014; Mühlig et al. 2014). Auch die Betrachtung der Adipositas im Kindes- und Jugendalter als chronische Erkrankung, wie sie Disse et al. diskutieren, impliziert die Notwendigkeit einer langfristigen Therapie (Disse und Zimmer 2014). Die Ergebnisse der Studien von Wilfley et al. und Deforche et al. unterstützen diese Theorie: In beiden Studien wurde in einem kontrollierten Setting ein besserer Erfolg durch Patienten erreicht, die im Anschluss an die Therapie an einem Aufrechterhaltungsprogramm teilnahmen (Deforche et al. 2005; Wilfley et al. 2007).

Die Ergebnisse der vorliegenden Studie wie auch die der EvAKuJ-Studie stützen diese Theorie dennoch nur in geringem Maße. Eine Assoziation zwischen längerer Therapiedauer und höherer Gewichtsreduktion lässt sich zwar vermuten, sollte jedoch in zukünftigen Studien gesichert werden. Sollten sich die Vermutungen bestätigen, empfiehlt sich auf lange Sicht der Ersatz kurzfristig angelegter Programme durch langfristige, die ggf. mit speziellen Aufrechterhaltungsprogrammen einhergehen.

Neben der Therapiedauer ist auch die Länge der postinterventionellen Zeit eine wesentliche Stärke der vorliegenden Studie. Grund hierfür ist, dass sich die vorliegende Arbeit mit der Entwicklung von Langzeitverläufen in der Adipositastherapie beschäftigt. Über die langfristige Entwicklung noch Jahre nach therapeutischen Interventionen ist dabei heute noch verhältnismäßig wenig bekannt. Die durchschnittliche postinterventionelle Zeit dieser Studie lag bei 4,1 Jahren, das Durchschnittsalter zum Follow-up-Zeitpunkt T2 bei 19,3 Jahren. Der kleinste postinterventionelle Zeitraum betrug 1,0 Jahre, der längste 9,0 Jahre. Im Vergleich liegt der gewählte mindeste Nachbeobachtungszeitraum in der Cochrane-Analyse nach Oude Luttikhuis sowie der Metaanlyse nach Mühlig et al. bei 6 Monaten und in der EvAKuJ-Studie sowie der Analyse von Wiegand et al. bei 2 Jahren. Der vorliegenden Arbeit vergleichbare Follow-up-Zeiten finden sich in einer Studie von Reinehr et al. mit einer Nachbeobachtungszeit von 4 Jahren (Reinehr et al. 2010). Die längsten uns bekannten Nachbeobachtungszeiten finden sich in einer Analyse von Epstein et al. mit einem Zeitraum von etwa 9 Jahren (Epstein und Valoski und et al. 1990). Längere Nachbeobachtungszeiten sind auch insofern von Bedeutung, als diese, wie in der vorliegenden Studie, den Übergang ins Erwachsenenalter miteinbeziehen können. Grund hierfür ist, dass kindliche Adipositas - im Vergleich zur Adipositas Erwachsener - wesentlich seltener mit Komorbiditäten assoziiert ist (Cole et al. 2000). Dagegen kommt es häufig zu einer Übertragung ins Erwachsenenalter mit entsprechenden Konsequenzen (Lloyd et al. 2010). 
Gerade um diese Entwicklung zu verhindern, ist die Kenntnis von Prädiktoren, die mit besseren Erfolgen assoziiert sind, folglich von großer Bedeutung.

\subsubsection{Beendigung der Therapie}

Der häufigste Grund für die Beendigung der Therapie war, dass der Patient selbst keine Fortführung wünschte $(41,4 \%$ der Patienten). An zweiter Stelle folgte ein mangelnder Therapieerfolg (13,3 \%), darauf ein zu weiter Anfahrtsweg (11,7 \%) und darauf ein disharmonisches Verhältnis zu den betreuenden Therapeuten (9,4 \%).

Letztere Angaben finden sich auch in einer Untersuchung von Barlow et al. wieder: Ausgewertet wurde eine retrospektive Elternbefragung von 43 Familien, die eine Adipositastherapie vorzeitig beendet hatten (Barlow und Ohlemeyer 2006). Bei der Frage nach dem Grund für das Therapieende gaben $37 \%$ an, Therapie und Therapeuten entsprächen nicht ihren Vorstellungen. $23 \%$ nannten eine zu große Entfernung vom Heimatort. In diese Untersuchung gingen dabei nur Patienten ein, für die die Teilnahme an einem Therapieprogramm angedacht war, die jedoch maximal zwei Termine wahrgenommen hatten. Insofern ist die Angabe einer Beendigung aufgrund eines mangelnden Therapieerfolgs (ohne Erhalt einer adäquaten Therapie) hier nicht zu erwarten.

Skelton et al. untersuchten im Rahmen einer Metaanalyse ebenfalls die Gründe für das Ausscheiden von Patienten aus Therapieprogrammen (Skelton und Beech 2011). Es zeigte sich, dass gerade Patienten mit höhergradiger Adipositas sowie Vorliegen von Komorbiditäten und Verhaltensauffälligkeiten dazu neigten, eine Therapie vorzeitig zu beenden.

In der vorliegenden Studie zeigte sich weiterhin ein signifikanter Unterschied in der Gewichtsreduktion zwischen den Gruppen: Wie zu erwarten zeigten Patienten, die die Therapie aufgrund eines Therapieerfolgs beendeten hatten, die höchsten Reduktionen. Patienten, die die Therapie auf eigenen Wunsch, aufgrund von Zeitmangel seitens des Patienten selbst sowie aufgrund von mangelndem Erfolg abbrachen, nahmen im Durchschnitt sogar an Gewicht zu. Damit scheint der Patientenwunsch einen großen Einfluss auf die Limitierung wie auch den Erfolg der Therapie zu haben. Im Vergleich konnten Patienten, die die Therapie aufgrund von Zeitmangel seitens der Eltern beendeten, die zweithöchsten Erfolge erzielen. Diese Ergebnisse implizieren, dass eine hohe kindliche Motivation für den Therapieerfolg von größerer Bedeutung ist als eine hohe elterliche Motivation. Betrachtet man das Alter der Patienten bei Beendigung der Therapie, so liegt dies im Schnitt bei 14,7 Jahren. Es ist gut vorstellbar, dass gerade für diese jugendliche Altersklasse, die von zunehmender Selbstbestimmtheit in Bezug auf Ess- und Bewegungsverhalten geprägt ist, die Patientenmotivation im Verhältnis zur Elternmotivation an Bedeutung gewinnt (Barlow 2007). Accurso et al. kamen ebenso in einer Untersuchung von 50 acht- bis zwölfjährigen Patienten zu dem Ergebnis, dass die kindliche Motivation für die erreichte Gewichtsreduktion von größerer Bedeutung ist als die elterliche (Accurso et al. 2014).

Insgesamt scheinen für eine Fortführung der Therapie die Patientenmotivation, organisatorische Aspekte sowie das Verhältnis zu den Therapeuten eine große Rolle zu spielen. Ein möglicher Ansatz zum Umgang mit diesen Faktoren ist eine regelmäßige Erfassung von Patientenmotivation und -zufriedenheit (vlg. Kapitel „Bewertung der Therapie“). Bei etwaigen (auch organisatorischen) Unstimmigkeiten oder einer nicht ausreichenden Motivation sollte in Interaktion mit dem Patienten und seiner Familie der Versuch einer gemeinsamen Problemadressierung und Lösungsfindung unternommen werden. Skelton et al. schlagen beispielsweise als mögliche zukünftige Option zur Umgehung langer Anfahrtswege die Einführung von Internet-basierten Video-Terminen vor (Skelton und Beech 2011). Zusammenfassend könnte durch individuelle und situationsbezogene Maßnahmen eine Optimierung der Therapieergebnisse mit einer Verringerung der Zahl frühzeitig ausscheidender Patienten erreicht werden. 


\subsubsection{Bewertung der Therapie}

Die Patienten hatten die Möglichkeit, freie positive und negative Kritik bezüglich der einzelnen Therapiemodalitäten zu äußern. Die geäußerten Kritikpunkte variierten jedoch stark, sodass keine repräsentativen Ergebnisse erhoben werden konnten.

Dagegen zeigten sich signifikante Zusammenhänge für die kurzfristige und gesamte skalarische Bewertung (1 (positiv) bis 5 (negativ)) der einzelnen Disziplinen: Ein höherer Profit von ärztlicher Betreuung und Ernährungsberatung sowie eine höhere Zufriedenheit mit der Ernährungsberatung waren mit einem größeren Gewichtsverlust assoziiert. Für die Zufriedenheit mit der ärztlichen Betreuung konnte ebenfalls ein - jedoch nicht signifikanter Zusammenhang dargestellt werden. Es muss jedoch hinzugefügt werden, dass Patienten, die bessere Bewertungen der ärztlichen Betreuung und Ernährungsberatung vornahmen auch signifikant häufiger ihre körperliche bzw. vereinssportliche Aktivität steigerten. Es bleibt dabei zu diskutieren, ob die höheren Erfolge in der Gewichtsreduktion möglicherweise ursächlich auf diese körperliche Aktivität zurückzuführen ist. Dagegen spricht jedoch, dass eine Aktivitätssteigerung nur kurzfristig mit einer signifikant höheren Gewichtsreduktion assoziiert war, während dies für die Bewertung der Therapie auch über den Gesamtverlauf galt.

Für die psychologische Betreuung konnten in dieser Hinsicht keine signifikanten Zusammenhänge erfasst werden. Es muss jedoch bedacht werden, dass nur 72,1 \% der eingeschlossenen Patienten eine psychologische Therapie erhalten hatten. Damit liegt auch nur ein geringerer Datensatz mit einer geringeren Wahrscheinlichkeit statistisch aussagekräftiger Ergebnisse vor.

In einer Studie von Togashi et al. wurde ebenfalls die Patientenzufriedenheit in Abhängigkeit vom Therapieerfolg erfasst (Togashi et al. 2002). Für 276 Patienten, die zwischen 1976 und 1992 eine Adipositastherapie im Kindes- und Jugendalter erhalten hatten, erfolgte eine erneute Datenerhebung im Jahr 1998. Die Gewichts- und Größendaten zum Follow-up-Zeitpunkt beruhen dabei auf Selbstangaben. Im Gegensatz zur vorliegenden Studie wurden keine separaten Bewertungen für die verschiedenen Therapiedisziplinen erhoben. Auch in dieser Studie zeigten sich Patienten, die eine höhere Zufriedenheit mit der Therapie angaben, auch signifikant erfolgreicher.

Eine höhere Zufriedenheit mit der Therapie scheint also zu höheren Gewichtsreduktionen zu führen. Im klinischen Alltag könnte man diesen Ergebnissen durch ein regelmäßiges Abfragen der Patientenzufriedenheit durch die betreuenden Therapeuten begegnen. Auf eventuelle Unzufriedenheiten könnte - in einem realistischen Rahmen - mit einer gemeinsamen Lösungsfindung reagiert werden. Dennoch müssen die Ergebnisse der vorliegenden Studie wie auch der Untersuchung von Togashi et al. (wie bereits im Kapitel „Datenerhebung“ besprochen) aufgrund der Selbstangaben kritisch betrachtet werden.

\subsubsection{Untersuchung des Einflusses therapieunabhängiger Parameter auf die BMI- SDS-Reduktion}

\subsubsection{Motivation vor Therapiebeginn und Therapieziel}

Es ist auffällig, dass die Motivation zur Therapie mit 42,2 \% in der Mehrzahl der Fälle von der Mutter der Patienten ausging, während der Vater nur in 5,5 \% der Fälle involviert war. Ein möglicher Grund hierfür ist das auch heute noch präsente Rollenkonzept der sozialen Mutterschaft mit geringerer Einbeziehung des Vaters in Kinderernährung und -erziehung (Blossfeld 1995; Craig 2006). In weiteren 14 \% ging die Motivation vom Kind selbst aus.

Es zeigte sich jedoch kein signifikanter Unterschied in den Gewichtsreduktionen abhängig von der Person oder Instanz, die die Therapie motivierte. Es bleibt fraglich, ob es für die Gewichtsreduktion tatsächlich nicht von Bedeutung ist, von welcher Person die Therapie motiviert wird, oder ob in der vorliegenden Studie ein Zusammenhang lediglich nicht erfasst werden konnte. 
Die Ergebnisse des vorherigen Absatzes „Beendigung der Therapie“ implizieren, dass gerade die kindliche Motivation sehr wohl einen Einfluss auf das Therapieergebnis nimmt. Accuros et al. kamen in ihrer Untersuchung jedoch zu dem Schluss, dass die vor Therapiebeginn erfasste Motivation weniger Bedeutung für den Therapieerfolg hat als die im Therapieverlauf erfasste Motivation (Accurso et al. 2014). In einer Untersuchung von Kirschenbaum et al. erreichten sogar diejenigen Kinder, die im Vorhinein die höchsten Motivationswerte angaben, die geringsten Gewichtsreduktionen (Kirschenbaum et al. 1984).

Es liegen noch keine einheitlichen Methoden zur Erfassung der Motivation von Patient und Familie vor. Im Rahmen des „Obeldicks“-Therapieprogramms zeigte sich ein besserer Therapieerfolg für Patienten, die bereits vor Therapiebeginn ein achtwöchiges Sportprogramm absolvierten sowie ein Ernährungsprotokoll über drei Tage führten (Dobe et al. 2011; Reinehr et al. 2003). Dies wird im Rahmen des Programms als Indikator für eine ausreichende Therapiemotivation gewertet, da die Familien bereits an dieser Stelle demonstrieren, dass sie den nötigen organisatorischen und zeitlichen Aufwand für die Durchführung einer Adipositastherapie in Kauf nehmen. Dies scheint eine sinnvolle, wenngleich relativ aufwändige Methode zur Motivationserfassung zur sein. Insgesamt sind für die adäquate Erfassung dieses Parameters zukünftig noch weitere Untersuchungen nötig.

Bezüglich der Erwartungen an die Therapie stand mit 45 Angaben (35,1\% der Patienten) die Ernährungsschulung im Vordergrund. Erst an zweiter Stelle folgte mit 23 Angaben (18\%) die Abklärung medizinischer Ursachen bzw. Folgen. Bedenkt man, dass die Hauptintention hinter der angestrebten Gewichtsreduktion im Rahmen einer Adipositastherapie die Verringerung von psychischen und physischem Komorbiditäten ist, erscheint dieser Prozentsatz recht gering (Wabitsch und Kunze 2015).

Gleichzeitig strebten in Bezug auf das Gewicht 94,1 \% der Patienten eine Gewichtsreduktion an, 2,3\% eine Stabilisierung. Zwölf Patienten hatten dabei bereits im Vorhinein konkrete Vorstellungen bezüglich des angestrebten Gewichtsverlusts. Insgesamt erscheinen diese Vorstellungen im Vergleich mit den durchschnittlich erreichten Reduktionen wenig realistisch. So wurde häufig eine Gewichtsnormalisierung, in drei Fällen sogar ein Gewicht unterhalb der 50. Perzentile angestrebt.

In diesem Zusammenhang fiel auf, dass Patienten mit dem Ziel einer Gewichtsreduktion im Gegensatz zu einer Gewichtsstabilisierung insgesamt im Durchschnitt eine um bis zu 0,79 höhere BMI-SDS-Reduktion erreichten. Der durchschnittliche BMI-SDS bei Therapiebeginn lag für erstere Patienten bei 2,37, für letztere bei 2,67. Auch in diesem Fall lässt sich eine Assoziation mit einer höheren Motivation und in der Folge höheren Gewichtsreduktionen vermuten. Ein Zusammenhang kann bei fehlender Signifikanz jedoch lediglich angenommen werden. Dagegen konnten die zwölf Patienten mit konkreter Vorstellung von der angestrebten Gewichtsreduktion insgesamt um 0,71 höhere BMI-SDS-Reduktionen erreichen. Dieses Ergebnis ist im Hinblick auf die unrealistischen Zielvorstellungen überraschend. Zu bedenken ist auch, dass diese Patienten auch ein signifikant intensiveres Therapiekonzept erhalten haben sowie signifikant höhere Profitangaben von der ärztlichen Behandlung machten. Zusammenhänge der Therapieerfolge zum Therapiekonzept sind unwahrscheinlich (siehe Kapitel „Therapiekonzept“). Für Profitangaben und Vorhandensein eines Zielgewichts bleibt dagegen zu diskutieren, ob der höhere Therapieerfolg des einen Parameters womöglich lediglich über den jeweils anderen Parameter vermittelt wird.

Das Thema der Patientenerwartungen an die Gewichtsreduktion ist für Erwachsene bereits gut untersucht. Dabei scheinen zu hoch gesteckte Ziele ein häufiges Phänomen zu sein (Fabricatore et al. 2007; Foster et al. 1997; Linde et al. 2004). Nach der Theorie des „Falsche-HoffnungSyndroms" sollte jedoch zwischen erreichbaren und nicht erreichbaren Zielen unterschieden werden (Polivy 2001). Das Anstreben letzterer ist dabei zu vermeiden, um dem vorprogrammierten Versagen mit konsekutiver Enttäuschung vorzubeugen. Überträgt man diesen Gedanken auf die Adipositastherapie, empfiehlt sich die Wahl eines möglichst realistischen und erreichbaren Zielgewichts. Die Ergebnisse einiger Untersuchungen stützen diese These, so konnte zum Beispiel eine Assoziation zwischen unrealistisch hohen Erwartungen an die Gewichtsreduktion und einem erneuten Gewichtsanstieg nach primärer Abnahme 
beobachtet werden (Byrne et al. 2003; Olson et al. 2012). Dennoch existieren diverse Untersuchungen, die keinerlei negativen Einfluss unrealistisch niedriger Zielgewichte auf Gewichtsentwicklung oder Psyche der Patienten erfassen konnten (Fabricatore et al. 2007; Jeffrey et al. 1998). Wie auch in der vorliegenden Studie ergaben sich in einer Untersuchung von 302 erwachsenen Frauen nach Linde et al. langfristig sogar höhere Gewichtsreduktionen für Teilnehmerinnen mit unrealistisch hochgesteckten Zielen (Linde et al. 2004). Damit ist die weitläufige Meinung, Patienten solle von überhöhten Vorstellungen ab- und zu kleinen, erreichbaren Etappenzielen zugeraten werden (Barlow 2007; Mühlig et al. 2014; Westenhoefer 2001), zumindest zu überdenken. Eine abschließende Klärung dieses Aspekts gestaltet sich jedoch im Rahmen der aktuellen Studienlage, die sich insbesondere auf Erwachsene konzentriert, schwierig.

\subsubsection{Alter bei Therapiebeginn}

In die vorliegende Studie wurden Kinder im Alter von 11 bis 17 Jahren eingeschlossen (Durchschnittsalter: 13,6 Jahre). Durch Korrelation der Werte für das Alter bei Therapiebeginn und die BMI-SDS-Reduktion über den Therapiezeitraum wurde eine signifikant bessere Reduktion für jüngere Kinder festgestellt. Es ist in diesem Zusammenhang jedoch zu bedenken, dass ein jüngeres Alter in der vorliegenden Studie mit einer stärkeren Steigerung der körperlichen Aktivität sowie einem niedrigeren BMI-SDS bei Therapiebeginn einherging. Beide Faktoren waren ebenfalls mit einer höheren Gewichtsreduktion über den Therapiezeitraum verknüpft. Es bleibt zu diskutieren, inwiefern die Parameter sich gegenseitig sowie den Erfolg der Gewichtreduktion beeinflussen.

In ihrer APV-Datenbank-Analyse untersuchten Wiegand et al. den Einfluss des Alters bei Therapiebeginn für einen Zeitraum von zwei Jahren nach Behandlungsbeginn (Wiegand et al. 2014). Auch hier ließ sich eine signifikant bessere Gewichtsabnahme von 5- bis 11-jährigen im Vergleich zu 14- bis 15-jährigen Patienten feststellen. Zu bedenken ist allerdings, dass letztere Gruppe sich dabei über beinahe den gesamten Therapiezeitraum im durchschnittlichen Alter der Pubertät bewegt (SE Anderson und Must 2005). Für diese Phase sind generell schwierigere Therapiebedingungen zu erwarten, was ein Erklärungsansatz für die geringeren Therapieerfolge sein könnte (Barlow 2007; Reinehr et al. 2010). Auch Reinehr et al. kamen in einer Fünf-JahresFollow-up-Studie zu ähnlichen Ergebnissen: Bei Einschluss von 4- bis 16-jährigen Kindern und Jugendlichen waren die höchsten Reduktionen nach fünf Jahren für Kinder $<8$ Jahren, die niedrigsten für Kinder $>13$ Jahren zu finden (Reinehr et al. 2010). Schließlich ließ sich in der EvAKuJ-Studie mit Einschluss 8- bis 16-jähriger Patienten (Durchschnittsalter: 12,2) für den im

Durchschnitt 5,8 Monate dauernden Therapiezeitraum ebenfalls eine bessere Gewichtsreduktion für jüngere Patienten verzeichnen (Böhler et al. 2012).

Ein möglicher Erklärungsansatz für dieses Phänomen ist zum einen ein größerer elterlicher Einfluss mit möglicher positiver Auswirkung auf den Therapierfolg in jüngeren Altersgruppen (Barlow 2007; Epstein und Wing 1987; Reinehr et al. 2010). Ein weiterer möglicher Grund ist eine höhere Beeinflussbarkeit jüngerer Kinder bezüglich gesundheitsbezogener Verhaltensweisen mit leichterer Festigung neuer Gewohnheiten. (Epstein und Wing 1987; Wiegand et al. 2014). Im Hinblick auf die Ergebnisse der vorliegenden Studie könnte insofern die höhere sportliche Aktivität jüngerer Patienten gerade auf das jüngere Alter zurückzuführen sein. Dagegen ist vor allem bei Eintritt in die Pubertät mit einer Verringerung der Compliance und des elterlichen Einflusses zu rechnen (Barlow 2007; Reinehr et al. 2010).

Die postinterventionellen Ergebnisse sowie die Ergebnisse über den Gesamtverlauf stellen sich in der vorliegenden Studie genau invers dar: Während es über den Therapieverlauf jüngere Patienten waren, waren es langfristig und insgesamt die bei Therapiebeginn älteren Patienten, die eine signifikant höhere Gewichtsreduktion erreichten.

Tatsächlich konnten auch für diesen Zusammenhang durch Reinehr et al. ähnliche Ergebnisse aufgestellt werden: Während Kinder in der Altersgruppe von 8 bis 10 Jahren über den Therapieverlauf erfolgreicher waren als Kinder im Alter von 11 bis 12, kam es langfristig zu einer Umkehr: Auf lange Sicht erreichten die 11- bis 12-jährigen im Vergleich die höheren Gewichtsreduktionen (Reinehr et al. 2010). Dennoch waren es die < 8-jährigen, die über den gesamten Zeitraum die höchsten Reduktionen erzielten. Auch in einer Untersuchung von Braet 
et al. mit Einschluss von Kindern im Alter von 7 bis 17 Jahren (Durchschnittsalter: 12,7) waren ältere Kinder erfolgreicher: Kinder und Jugendliche $>12$ Jahren konnten höhere Gewichtsreduktionen erreichen als < 12-jährige (Braet 2006). In der EvAKuJ-Studie ließ sich dagegen auf lange Sicht nach ITT-Analyse (bis zum Follow-up-Zeitpunkt nach zwei Jahren) kein Zusammenhang zwischen dem Alter zu Beginn der Therapie und der Gewichtsreduktion feststellen (Böhler et al. 2012).

Braet et al. vermuten, dass sich ein besseres Abschneiden älterer Kinder durch mit dem Alter zunehmende Kontrollmechanismen erklären lässt (Braet 2006). In der vorliegenden Studie kommt hinzu, dass die Patienten zum Follow-up-Zeitpunkt bereits durchschnittlich 19 Jahre alt waren und somit im Schnitt ein Übertritt ins Erwachsenenalter mit Ausklang der Pubertät stattgefunden hat. Dies stellt womöglich einen zusätzlichen unterstützenden Faktor für ein eigenverantwortliches und gesundheitsorientiertes Handeln dar. Dagegen sprechen wiederum die bereits genannten Ergebnisse von Reinehr et al., nach denen bei Therapiebeginn $>13$ jährige Patienten schlechter Abschneiden als bei Therapiebeginn $<8$-jährige (Reinehr et al. 2010). Zum Follow-up-Zeitpunkt (vier Jahre nach Therapiebeginn) ist erstere Gruppe folglich > 17 Jahre alt und entspricht damit im Alter in etwa dem Kollektiv der vorliegenden Studie, trotz der genannten Überlegungen schneidet jedoch die jüngere Gruppe besser ab.

Insgesamt scheint sich über den Therapieverlauf ein jüngeres Alter positiv auf den Therapieerfolg auszuwirken. Auch langfristig scheinen gerade sehr junge Altersgruppen (ca. unter acht Jahren, vgl. Untersuchungen von Wiegand et al. und Reinehr et al.) die höchsten Erfolge erzielen. Vergleiche mit den Daten der vorliegenden Arbeit sind bei fehlendem Einschluss dieser Altersklasse allerdings nicht möglich.

Weiterhin lassen sich langfristig aber auch für ein steigendes Alter mit Ausklang der Pubertät positive Effekte auf Kontrollmechanismen und Therapieerfolg vermuten.

In der Gesamtheit sind die Ergebnisse folglich noch etwas inkonsistent und bedürfen weiterer Forschung, wünschenswert wäre auch eine begleitende Erfassung der Pubertätsentwicklung.

\subsubsection{BMI-SDS bei Therapiebeginn}

Für den BMI-SDS zu Therapiebeginn konnte in der vorliegenden Studie ein signifikanter Zusammenhang mit der kurzfristigen BMI-SDS-Reduktion erfasst werden. Dabei erreichten Kinder und Jugendliche mit höherem Ausgangsgewicht über den Therapieverlauf geringere Gewichtsreduktionen. Auch langfristig ließ sich dieser Trend noch nachvollziehen, über den Langzeitverlauf zeigte sich ebenfalls noch eine geringe Korrelation. Bei fehlender Signifikanz ist ein langfristiger und gesamter Zusammenhang statistisch jedoch nicht gesichert. Zudem ist zu berücksichtigen, dass Kinder mit einem höheren BMI-SDS bei Therapiebeginn auch signifikant älter waren. Da ein höheres Alter bei Therapiebeginn kurzfristig ebenfalls mit einer geringeren Gewichtsreduktion assoziiert war, stellt sich die Frage, ob ein Zusammenhang für einen der Parameter - bei gleichzeitigem bestehen des jeweils anderen - lediglich vermittelt wird. Das weiterbestehen des Trends über den langfristigen und gesamten Verlauf lässt einen Zusammenhang zwischen BMI-SDS bei Therapiebeginn und Gewichtsreduktion jedoch zumindest vermuten.

Die Ergebnisse der vorliegenden Studie entsprechen denen der EvAKuJ-Studie: Auch hier zeigten sich Patienten mit niedrigerem Ausgangsgewicht ambulant kurz- und mittelfristig und stationär kurzfristig erfolgreicher. Langfristig konnten dagegen keine signifikanten Ergebnisse erhoben werden (Böhler et al. 2012).

In zwei weiteren Studien war dagegen auf lange Sicht ein höheres Ausgangsgewicht sogar mit höheren Reduktionen assoziiert: Zum einen traf dies für die Ergebnisse von Braet et al. zum Follow-up-Zeitpunkt zwei Jahre nach Therapiebeginn zu (Braet 2006). Weiterhin konnten auch Epstein et al. in ihrer Zehn-Jahres-Follow-up-Erhebung eine insgesamt höhere Gewichtsreduktion von Patienten mit höherem Ausgangsgewicht feststellen (Epstein et al. 1994). $\mathrm{Zu}$ berücksichtigen ist jedoch, dass die Größe des Patientenkollektivs der beiden letztgenannten Studien mit 122 (Braet 2006) bzw. 158 (Epstein et al. 1994) weit hinter denen der EvAKuJ-Studie mit 1916 (und in geringerem Ausmaß auch hinter dem der vorliegenden 
Arbeit mit 358) eingeschlossenen Patienten zurückliegt. Aus rein statistischer Sicht sind die erhobenen Ergebnisse damit vergleichsweise weniger repräsentativ.

Insgesamt scheint zumindest kurzfristig ein höheres Ausgangsgewicht mit einer geringeren Gewichtsreduktion assoziiert zu sein. Hieraus könnte man schließen, dass bereits bei geringerer Ausprägung stärkere Therapiemaßnahmen ergriffen werden sollten. Böhler et al. argumentieren jedoch, dass weniger übergewichtige Kinder und Jugendliche auch zu einem günstigeren Spontanverlauf neigen (Böhler et al. 2012).

Die Ergebnisse bezüglich der langfristigen Entwicklung sind dagegen noch relativ inkonsistent. Weitere Untersuchungen dieses Zusammenhangs in Zukunft wären wünschenswert.

\subsubsection{Diagnosen und Komorbiditäten}

Insgesamt lagen für $74 \%$ der Patienten adipositasbezogene Komorbiditäten bzw. Risikofaktoren vor. Zum größten Teil waren dies metabolische Veränderungen. Auch in der EvAKuJ-Studie wurde das Vorliegen von Komorbiditäten erfasst (Böhler et al. 2012). Dabei lag der Anteil von Patienten mit Hypertonie und Dyslipidämie jedoch deutlich höher, mit $32 \%$ bzw. $31 \%$ in der EvAKuJ- und 9 \% bzw. 9,5 \% in der vorliegenden Studie. Der durchschnittliche BMI zu Therapiebeginn lag dabei in der EvAKuJ-Studie bei 29,98, in der vorliegenden Studie bei 31,8. Die Vergleichbarkeit der Daten ist weiterhin eingeschränkt, da die Erhebung im Rahmen der EvAKuJ-Studie ausschließlich bei Therapiebeginn erfolgte (wobei die Erhebung aus Patientenakten und Arztbriefen in der vorliegenden Studie den kompletten Therapiezeitraum umfasst) und zudem nur für $73 \%$ der Patienten eine Blutdruck- und für $67 \%$ eine Lipidmessung erfolgte (Böhler et al. 2012).

Insgesamt liegt ein sehr hoher Prozentsatz an Patienten mit adipositasbezogenen Komorbiditäten vor. Die laut AGA empfohlene Indikation zur Adipositasschulung für jedes übergewichtige oder adipöse Kind mit adipositasbezogenen Komorbiditäten bedürfte folglich enormer Ressourcen (Wabitsch und Kunze 2015). Es ist jedoch zu berücksichtigen, dass das im SPZ behandelte Patientengut bereits einer gewissen Selektion hin zur extremen Adipositas unterliegt, sodass ein vergleichsweise höherer durchschnittlicher BMI-SDS mit einem folglich ebenfalls erhöhten Anteil an Patienten mit Komorbiditäten zu erwarten ist. Bei nicht ausreichenden Ressourcen könnte eine Orientierung des Therapiekonzepts an der Schwere der vorliegenden Komorbidität dennoch hilfreich sein (bspw. intensivere Therapie bei Vorliegen eines manifesten Diabetes im Vergleich zu einer Insulinresistenz).

Darüber hinaus wurde in der vorliegenden Studie beim Vorliegen einer Insulinresistenz über den Therapiezeitraum eine signifikant geringere Gewichtsreduktion erreicht. Ein möglicher Erklärungsansatz für dieses Phänomen ist, dass das Vorliegen einer Insulinresistenz in der vorliegenden Studie mit einem höheren BMI-SDS assoziiert war. Ein höherer BMI-SDS war kurzfristig wiederum ebenfalls mit einer geringeren Gewichtsreduktion verknüpft, sodass von einer Vermittlung über diesen Parameter ausgegangen werden kann.

Zudem war das Vorliegen einer psychiatrischen Diagnose über alle Zeiträume ebenfalls mit einer geringeren BMI-SDS-Reduktion assoziiert. Diese Ergebnisse waren jedoch statistisch nicht signifikant. Dagegen konnte kurzfristig ein signifikant geringerer Therapieerfolg von Patienten erreicht werden, die neben der psychologischen Betreuung im Rahmen der Adipositastherapie noch anderweitige kinder- und jugendpsychiatrische Interventionen erhalten hatten. Langfristig stellte sich dieser Zusammenhang jedoch nicht mehr dar. Auch an dieser Stelle ist die fehlende Erfassung einer Assoziation möglicherweise aber auch auf das vergleichsweise kleineren Patientenkollektiv zum Follow-up-Zeitpunkt zurückzuführen. Es muss weiterhin berücksichtigt werden, dass die Kinder und Jugendlichen mit Erhalt zusätzlicher psychiatrischer Interventionen bei Therapiebeginn auch signifikant älter waren und höhere BMI-SDS aufwiesen. Letztere Faktoren waren jedoch ebenfalls mit einer geringeren kurzfristigen BMI-SDS-Reduktion assoziiert, sodass sich die Frage stellt, inwieweit die Parameter sich gegenseitig und die Gewichtsreduktion kausal beeinflussen. Weiterhin ist zu bemängeln, dass die Erfassung von psychologischen Auffälligkeiten nicht für alle teilnehmenden Kinder erfolgte, was zu repräsentativeren Ergebnissen geführt hätte. 
Auch Epstein et al. kamen in einer Follow-up-Erhebung - auch langfristig - zu ähnlichen Ergebnissen: Zum Follow-up-Zeitpunkt zehn Jahre nach Therapiebeginn waren Kinder und Jugendliche, die psychiatrische Auffälligkeiten entwickelt hatten, weniger erfolgreich in der Kontrolle ihres Gewichts als nicht-psychiatrisch Erkrankte (Epstein et al. 1994).

Die dargestellten Ergebnisse legen nahe, dass eine erfolgreiche Gewichtsreduktion für Kinder mit psychiatrischen Erkrankungen schwerer zu erreichen ist. Weitere zukünftige Untersuchungen könnten weiterhin spezifische Erkrankungen als Negativprädiktoren für den Therapieerfolg identifizieren. Eine mögliche Reaktion auf diese Ergebnisse ist die Durchführung einer psychologischen/psychiatrischen Evaluation jedes Patienten vor Therapiebeginn, um so die Therapiestrategien speziell an den jeweiligen Patienten anpassen zu können.

\subsubsection{Geschlecht}

In Bezug auf das Geschlecht waren über alle drei untersuchte Zeiträume Jungen signifikant erfolgreicher als Mädchen. Es muss jedoch berücksichtigt werden, dass ein weibliches Geschlecht in der vorliegenden Arbeit auch mit höheren BMI-SDS sowie einem höheren Alter bei Therapiebeginn verknüpft war. Beide Faktoren gingen jedoch mit einer geringeren Gewichtsreduktion einher.

Diese Ergebnisse in Bezug auf das Geschlecht stehen im Einklang mit denen von Wiegand et al., in deren Zwei-Jahres-Follow-up-Erhebung Jungen im Vergleich zu Mädchen eine signifikant höhere Gewichtsreduktion erreichten (Wiegand et al. 2014). Im Rahmen der EvAKuJ-Studie konnte dieser Zusammenhang dagegen nur kurzfristig für ambulante Patienten erhoben werden (Böhler et al. 2012). Langfristig konnte für die ambulante Therapie kein Unterschied in Bezug auf das Patientengeschlecht mehr dargestellt werden. Bei der stationären Behandlung zeigten sich dagegen kurzfristig keine Unterschiede, während langfristig Mädchen signifikant erfolgreicher waren.

In verschiedenen anderen Studien lässt sich kein Unterschied für die Gewichtsreduktion zwischen den Geschlechtern feststellen (Braet 2006; Reinehr und Temmesfeld et al. 2007). Insgesamt entsteht auch im Hinblick auf die inkonsistenten Ergebnisse dieser genannten Studien der Eindruck, dass die höhere Gewichtsreduktion des männlichen Geschlechts eher über das jüngere Alter und den geringeren BMI-SDS bei Therapiebeginn vermittelt wird.

\subsubsection{Familiäre Situation und SES}

Der SES setzt sich aus verschiedenen Komponenten zusammen. Zwei Indizes zur Bestimmung des SES sind der Sozialschichtindex nach Winkler (Winkler und Stolzenberg 1999) sowie der Hollingshead-Index (Hollingshead 1975). Ersterer umfasst die Komponenten Bildung, berufliche Stellung sowie Einkommen. Letzterer schließt die Faktoren Familiensituation, Berufssituation, Bildungsstand und berufsbezogenes Prestige mit ein. Auch der Migrationsstatus wird oft im Zusammenhang mit dem SES besprochen (Röbl et al. 2013). Von diesen genannten Komponenten wurden in der vorliegenden Studie alle bis auf Einkommen und berufliches Prestige untersucht.

In Bezug auf die Schulbildung und Beruf wurde sowohl der Einfluss der kindlichen als auch der elterlichen Schulbildung überprüft. Dabei fiel auf, dass Patienten ohne im Vergleich zu Patienten mit abgeschlossener Schulausbildung (Haupt-/ Realschulabschluss oder Abitur) eine durchschnittlich schlechtere Reduktion über alle Zeiträume erreichten. Diese Ergebnisse waren jedoch nicht signifikant, sodass keine statistisch gesicherte Assoziation vorliegt. Ein Zusammenhang zwischen kindlichem Bildungsstand und Gewichtsreduktion konnte auch in einer Studie von Röbl et al. nachgewiesen werden: Eine Untersuchung von 62.147 Patienten mit einer Nachbeobachtungszeit von bis zu 3 Jahren nach einer Interventionszeit von sechs bis 24 Monaten ergab für Patienten, die eine Sonderschule besuchten, im Vergleich zu den übrigen Schulformen (Hauptschule, Realschule und Gymnasium) einen signifikant geringeren Therapieerfolg (Röbl et al. 2013).

Bezüglich der Berufssituation des Patienten bestand in der vorliegenden Studie kurzfristig ein signifikanter Unterschied in der Gewichtsreduktion. Dabei waren im Vergleich von Patienten mit bzw. ohne abgeschlossene Berufsausbildung erstere signifikant erfolgreicher. Auch über den 
Gesamtverlauf ließ sich ein, wenn auch nicht signifikanter, Zusammenhang darstellen. Ein direkter Vergleich dieser Ergebnisse mit anderen Untersuchungen ist nicht möglich, da sich unserer Kenntnis nach aktuell noch keine andere Studie mit dem Einfluss der beruflichen Situation des Patienten selbst auf die Gewichtsreduktion beschäftigt hat. Dagegen existieren verschiedene Untersuchungen bezüglich der elterlichen Berufssituation, die im folgenden besprochen werden.

Während sich für die elterliche Schulbildung kein Zusammenhang mit der kindlichen Gewichtsreduktion feststellen ließ, war eine fehlende mütterliche bzw. väterliche Berufsausbildung über alle drei Zeiträume mit einer geringeren Reduktion assoziiert. Ebenso waren sowohl niedrigere mütterliche als auch väterliche Wochenarbeitszeiten mit einer geringeren kurzfristigen, langfristigen und gesamten BMI-SDS-Reduktion verknüpft. Dennoch waren diese Ergebnisse statistisch nicht signifikant, sodass ein Zusammenhang lediglich angenommen werden kann. Auch an dieser Stelle bestehen Parallelen zu den Ergebnissen von Röbl et al.: Hier wurde eine signifikant höhere Gewichtsreduktion bei Kindern von vollzeitlich im Gegensatz zu teilzeitlich arbeitenden Eltern erhoben (Röbl et al. 2013). Im Rahmen der EvAKuJ-Studie erfolgte dagegen keine separate Untersuchung der Komponenten, sondern eine Erfassung des Sozialschichtindex nach Winkler, in den sowohl der elterliche Bildungsstand als auch der elterliche Beruf mit eingehen (Böhler et al. 2012). Dabei erzielten ambulante Patienten aus Familien, die einem niedrigeren SES zugeordnet wurden, langfristig geringere Erfolge in der Gewichtsreduktion. Eine genaue Vergleichbarkeit mit den vorliegenden Ergebnissen ist jedoch nicht möglich, da in den Sozialschichtindex neben den genannten Faktoren auch das elterliche Einkommen mit einfließt.

Bezüglich des Migrationshintergrunds konnte in der vorliegenden Studie kein Zusammenhang zur BMI-SDS-Reduktion erhoben werden. Dieses Ergebnis entspricht dem der EvAKuJ-Studie (Böhler et al. 2012). Dagegen erhoben Wiegand et al. in ihrer Analyse der APV-Datenbank zum Zwei-Jahres-Follow-up eine signifikant schlechtere Gewichtsreduktion für Patienten mit mindestens einem migrierten Elternteil (Wiegand et al. 2014). Zu diesem Ergebnis kamen auch Röbl et al. (Röbl et al. 2013). Auffällig in letzterer Studie war weiterhin, dass der Anteil von Patienten mit Migrationshintergrund mit elf Prozent wesentlich geringer war als in der Gesamtbevölkerung mit 25,4 \% (Röbl et al. 2013; Schenk et al. 2007). Auch in der vorliegenden Studie lag der Anteil mit insgesamt 15,7 \% unter dem der Gesamtbevölkerung. Nach Röbl ist ein Interpretationsansatz für diese Ergebnisse, dass Kinder und Jugendliche mit Migrationshintergrund seltener in Adipositastherapie-Programme eingegliedert werden. Ein möglicher Grund hierfür ist eine insgesamt erschwerte Inanspruchnahme medizinischer Betreuung, nicht zuletzt auch aufgrund häufig bestehender sprachlicher Barrieren. Um dem entgegenzuwirken ist eine Ressourcenausweitung beispielsweise im Rahmen von Sozialfürsorgestätten mit Erfassung von gefährdeten Kindern und Jugendlichen mit Zuführung in entsprechende Therapieprogramme nötig (Röbl et al. 2013).

Weiterhin erfolgte eine Untersuchung der Familien- bzw. Wohnsituation des Kindes. Dabei erreichten Patienten, die in einer Einrichtung lebten, im Gegensatz zu Patienten, die mit beiden oder einem Elternteil zusammen, in einer Patchwork-Familie, bei Pflegeeltern oder allein lebten geringere Reduktionen über alle Zeiträume. Dennoch sind diese Ergebnisse auch an dieser Stelle nicht signifikant und damit statistisch nicht gesichert. Eine mögliche Ursache für diesen Zusammenhang ist jedoch die häufige Unterbringung psychiatrisch auffälliger und innerhalb ihrer Familien nicht mehr führbarer Patienten in therapeutischen Einrichtungen. Wie im Absatz „Diagnosen und Komorbiditäten“ beschrieben, ging ein Vorliegen psychiatrischer Komorbiditäten in der vorliegenden Studie mit einer geringeren Gewichtsreduktion einher. Folglich besteht möglicherweise ein indirekter Zusammenhang der sich kausal auf das Vorhandensein psychiatrischer Begleiterkrankungen zurückführen lässt.

Es konnte bereits in mehreren Studien ein Zusammenhang zwischen der Familiensituation und dem Vorliegen von kindlicher Adipositas festgestellt werden. Dabei besteht für Kinder alleinerziehender sowie getrennt lebender Eltern eine höhere Wahrscheinlichkeit, adipös zu sein (Biehl et al. 2014; Chen und Escarce 2010). In der vorliegenden Studie konnte eine solche 
Familienstruktur jedoch nicht als Prädiktor für den Therapieerfolg erfasst werden. Dasselbe gilt die APV-Analyse nach Wiegand et al.: Auch hier konnte kein signifikanter Zusammenhang zwischen Familiensituation (Zusammenleben mit einem bzw. zwei Elternteilen) und der Gewichtsentwicklung dargestellt werden. In einer Arbeit von Wilfley et al. geht die Familienstruktur als Komponente des Hollingshead-Index mit ein: Anhand von 204 Patienten wurden die Erfolge verschiedener Erhaltungsprogramme nach stattgehabter Adipositastherapie untersucht. Vor Therapiebeginn erfolgte neben anderen Parametern eine Erfassung des SES (Wilfley et al. 2007). Während sich kurzfristig kein Zusammenhang zeigte, stellten sich zur Follow-up-Erhebung nach 2 Jahren für beide Erhaltungsprogramme Patienten mit niedrigerem SES weniger erfolgreich dar. Auch an dieser Stelle ist ein Vergleich aufgrund der übrigen in den Index eingehenden Faktoren jedoch schwierig.

Insgesamt scheint ein niedrigerer SES mit einem schlechteren Therapie-Outcome assoziiert zu sein. Es werden verschiedene Ursachen für diesen Zusammenhang diskutiert. Hierzu zählen ein geringeres Problembewusstsein in Bezug auf Übergewicht und Adipositas, begrenzte elterliche Ressourcen zur Unterstützung ihrer Kinder im Rahmen von Therapieprogrammen und mangelnde kognitive Fähigkeiten (Kersting und Clausen 2007; Röbl et al. 2013; Story et al. 2008). Für die berufliche Situation der Patienten ist ebenfalls zu diskutieren, ob die Adipositas ein Resultat des niedrigen SES ist oder ob andersherum auch die Adipositas selbst zu verminderten beruflichen Erfolgen führte. Dass ein vergleichsweise niedriger SES auch die Folge einer bestehenden Adipositas sein kann zeigten bereits Gortmarker et al. Im Jahr 1993 (Gortmaker et al. 1993).

Die Ergebnisse legen nahe, dass gerade für Patienten mit niedrigerem SES intensivere Therapieprogramme, insbesondere unter Einbindung der Familie, nötig sind. Auch eine Steigerung der Aufklärungsarbeit mit konsekutiver Erhöhung des Problembewusstseins könnte ein sinnvoller Schritt sein (Barlow 2007; Ebbeling et al. 2002). Schließlich ist anzunehmen, dass Familien mit niedrigerem SES häufig geringere Ressourcen aufweisen, um der kindlichen Adipositas zu begegnen. Es sollte vor Therapiebeginn eine Erfassung der Familienverhältnisse erfolgen. Bei instabilen Familiensituationen und geringen Ressourcen zur Unterstützung des Kindes empfiehlt sich gegebenenfalls eine Einbeziehung weiterer Modalitäten, wie etwa eines Kinder- und Jugendpsychiaters bzw. Kinder- und Jugendpsychotherapeuten, eine Erziehungsberatung oder in Kooperation mit dem Jugendamt der Einsatz eines Familienhelfers (BABELUGA o.J.).

\subsubsection{Elterlicher Gewichtsstatus}

In Bezug auf das Elterngewicht konnte sowohl für das mütterliche als auch für das väterliche Gewicht über den Therapieverlauf ein Zusammenhang zur Gewichtsentwicklung festgestellt werden. In beiden Fällen lag jedoch keine statistische Signifikanz vor, sodass nur eine mutmaßliche Assoziation besteht. Langfristig und über den Gesamtverlauf ließ sich keine Korrelation darstellen, wobei dies auch auf das vergleichsweise kleinere Kollektiv über diese Zeiträume zurückzuführen sein könnte.

Ein Zusammenhang zwischen dem Vorliegen von kindlichem Übergewicht mit einem überhöhten Elterngewicht konnte bereits mehrfach nachgewiesen werden (Röbl et al. 2008; Whitaker et al. 1997). In einer Studie von Fröhlich et al. mit Untersuchung von 111 Kindern erwies sich der familiäre Gewichtsstatus (unter Einschluss von mütterlichem sowie Gewicht der Geschwister) ebenfalls als Prädiktor für die Gewichtsreduktion: Bei einer Erfolgsdefinition von einer Reduktion des Ausgangsgewichts um mindestens $5 \%$ (entsprechend einer BMI-SDSReduktion von 0,2), waren Kinder mit niedrigerem familiären Gewichtsstatus zum Follow-upZeitpunkt nach einem Jahr signifikant erfolgreicher (Fröhlich et al. 2011). Dies entspricht damit zeitlich in etwa den kurzfristigen Ergebnissen der vorliegenden Untersuchung.

Epstein et al. konnten im Gegensatz zur vorliegenden Studie auch für den Langzeitverlauf einen Zusammenhang zwischen Elterngewicht und kindlichem Therapieerfolg nachweisen, wobei ein höheres Elterngewicht mit einer geringeren kindlichen Gewichtsreduktion zehn Jahre nach Therapiebeginn assoziiert war (Epstein et al. 1994). 
Mögliche Gründe für den geringeren Therapieerfolg von Kindern adipöser Eltern sind ein Zusammenspiel von genetischen und Umweltfaktoren mit Beeinflussung der kindlichen Gewichtsreduktion (Epstein und Wing 1987). So ist beispielsweise zu erwarten, dass übergewichtige und adipöse Eltern selbst adipogene Verhaltensweisen aufweisen, die sie in Ihrer Funktion als Vorbild und Autoritätsperson auf ihre Kinder übertragen (Roth et al. 2002; van der Horst et al. 2007). In diesem Zusammenhang scheint im Rahmen einer Adipositastherapie immer auch die Erfassung des Elterngewichts sinnvoll. Wie auch im Fall des SES könnte eine stärkere Einbindung der Familie mit entsprechenden Elternschulungen zu einer Steigerung der Therapieeffekte beitragen.

\subsubsection{Untersuchung des Einflusses sonstiger Parameter auf die BMI-SDS- Reduktion}

\subsubsection{Sportliche Aktivität}

Es konnte über den Gesamtzeitraum eine signifikant höhere Reduktion von Patienten, die um den Therapiezeitraum einen Vereinssport ausübten, im Vergleich zu Patienten ohne sportliche Betätigung erreicht werden. Zu bedenken ist jedoch, dass Patienten mit Aktivität im Sportverein durchschnittlich signifikant jünger waren. Da ein jüngeres Alter bei Therapiebeginn kurzfristig ebenfalls mit einer höheren BMI-SDS-Reduktion assoziiert war, stellt sich die Frage, ob tatsächlich beide Faktoren einen kausalen Einfluss auf die Gewichtsentwicklung nehmen.

Für einen Einfluss der sportlichen Aktivität spricht, dass über alle Zeiträume (mit Ausnahme der vereinssportlichen Aktivität zum Follow-up-Zeitpunkt im Bezug auf die langfristigen BMI-SDSReduktionen) Patienten mit vereinssportlicher Betätigung höhere durchschnittliche Reduktionen als Patienten erreichten, die keinen Sport ausübten. Diese Ergebnisse sind jedoch nicht signifikant und damit statistisch nicht gesichert sind.

Für eine sportliche Aktivität außerhalb eines Vereins konnten keine wesentlichen Zusammenhänge erhoben werden. Diese Ergebnisse stützen die im Kapitel „Entwicklung des Freizeitverhaltens" geäußerte Theorie, dass Sportarten in einem kontrollierten, vorgegebenen Setting, wie Vereinssportarten, sich effektiver auf die Gewichtsreduktion auswirken. Generell scheint eine intensivere Förderung von Kindern und Jugendlichen ohne sportliche Betätigung mit dem Versuch einer Motivationssteigerung in diesem Bereich sinnvoll. Anhand der dargestellten Ergebnisse bieten sich Trainingseinheiten in einem kontrollierten Setting im Gegensatz zu freier Betätigung der Kinder und Jugendlichen an (vgl. Ergebnisse Epstein et al. (Epstein et al., 1994), siehe Kapitel „Entwicklung des Freizeitverhaltens“). Bezüglich der Stundenzahl der sportlichen Aktivität pro Monat konnte ebenfalls kein signifikanter Zusammenhang erhoben werden. Auch in diesem Zusammenhang ist dies womöglich auf die Selbstangaben der Patienten mit Schätzung der Dauer der sportlichen Betätigung zurückzuführen.

\subsubsection{Regelmäßiges Wiegen}

Bezüglich eines regelmäßigen Wiegens konnte kein Zusammenhang mit dem Erfolg der Gewichtsreduktion festgestellt werden. Diese Ergebnisse sind konsistent mit denen einer Untersuchung von Neumark-Sztainer et al. (Neumark-Sztainer et al. 2006): In einer Longitudinalstudie über einen Zeitraum von fünf Jahren wurden 2516 Jugendliche bezüglich ihrer Ernährungs- und Wiegegewohnheiten sowie anthropometrischer Daten befragt. Es zeigte sich kein Zusammenhang zwischen einer Gewichtsentwicklung über diesen Zeitraum und regelmäßigem Wiegen durch den Patienten. Für jüngere weibliche Teilnehmer konnte stattdessen sogar eine Assoziation zwischen der Wiegehäufigkeit und einem Gewichtsanstieg beobachtet werden. Außerdem war bei den weiblichen Teilnehmern ein Zusammenhang zwischen ungesunden Gewichtskontrollmechanismen und der Gewohnheit, sich regelmäßig zu wiegen, festzustellen. Wie auch in der vorliegenden Studie beruhen diese Ergebnisse jedoch auf Selbstangaben, was sich negativ auf deren Reliabilität auswirkt.

VanWormer et al. konnten dagegen in einem Review mit Einschluss von zwölf Studien zum Einfluss der Wiegegewohnheiten Erwachsener einen positiven Einfluss regelmäßigen Wiegens 
auf die Gewichtsentwicklung feststellen (VanWormer et al. 2008): Dabei lag der erhobene BMI von Teilnehmern, die sich regelmäßig täglich oder wöchentlich wogen, um $1-3 \mathrm{~kg} / \mathrm{m}^{2}$ unter dem der übrigen Teilnehmer. Zu bemängeln ist jedoch, dass sich nur drei dieser zwölf Studien ausschließlich mit dem Effekt regelmäßigen Wiegens beschäftigten. Die übrigen erfassten die Wiegegewohnheiten nur als Teilaspekt eines umfangreicheren Therapieprogramms. Die Ergebnisse dieser drei Studien waren dagegen wenig valide und widersprüchlich. Weiterhin ist fraglich, inwiefern die Resultate Erwachsener auf Kinder und Jugendliche übertragbar sind. Gerade in der emotionalen Pubertätszeit, in der Selbstbild und Selbstwertgefühl leicht durch externe Einflüsse manipuliert werden können, lässt sich eine begrenzte Vergleichbarkeit mit den Auswirkungen auf Erwachsene annehmen.

Nach Barlow et al. kann das regelmäßige Wiegen als Gewichtskontrollmechanismus im Kindesund Jugendalter sowohl mit positiven als auch mit negativen (z. B. bestrafende Verhaltensweisen bei Gewichtsanstieg, Vernachlässigung gesunder Verhaltensweisen zu Gunsten einer höheren Gewichtsreduktion) Folgen einhergehen (Barlow und Dietz 1998). Zukünftige Untersuchungen sind nötig, um eindeutigere Aussagen zu diesem Aspekt machen zu können.

\subsection{Ausblick: „Wer braucht was?“}

In der vorliegenden Studie konnten verschiedene patientenbezogene Prädiktoren für den Verlauf einer Adipositastherapie erhoben werden. Faktoren, die mit einer geringeren Gewichtsreduktion assoziiert waren, waren das Fehlen eines konkreten Zielgewichts, ein höheres Alter und ein höherer BMI bei Therapiebeginn, ein niedriger SES sowie ein hohes Elterngewicht, das Vorhandensein von physischen oder psychischen Komorbiditäten (konkret: das Vorliegen einer Insulinresistenz oder Erhalt einer Psychotherapie unabhängig von der Adipositastherapie) sowie eine geringe sportliche Aktivität. Auch eine Unzufriedenheit mit der erhaltenen Therapie war mit geringeren Gewichtsreduktionen verknüpft. Das Vorhandensein solcher Einflussfaktoren auf die Gewichtsreduktion führt zwangsläufig zu dem Schluss, dass nicht jedes Kind unter der gleichen Therapie auch gleich erfolgreich ist, sondern das Therapieergebnis immer von verschiedenen Faktoren beeinflusst wird. Dies wiederum legt nahe, dass nur mithilfe einer Individualisierung von Therapiemaßnahmen unter Anpassung an die jeweiligen persönlichen Umstände ein optimales Therapieergebnis erreicht werden kann.

Betrachtet man die aktuellen Therapieprogramme in Deutschland, so fällt auf, dass auch die BABELUGA-Baustellen-Methode der Charité-Universitätsmedizin Berlin diesen Ansatz aufgreift: Vor Therapiebeginn erfolgt hier eine Erfassung der Parameter BMI, familiäre Situation, psychosoziale Problematik, Komorbidität, Leidensdruck, Veränderungsmotivation, Zielplanung sowie der Aktivität des Lebensstils (eine genaue Darstellung findet sich auf Seite 15). Anhand der erhobenen Daten erfolgt eine Einteilung der Patienten in Gruppen von A bis D, wobei Gruppe A die günstigsten Therapiebedingungen aufweist, Gruppe D die ungünstigsten. Vergleicht man die Prädiktoren für den Therapieerfolg in der vorliegenden Studie mit den Parametern der BABELUGA-Methode, so fallen deutliche Parallelen auf: Das Vorhandensein von den in der vorliegenden Studie erfassten Negativprädiktoren ist auch mit einem Abstieg in ungünstigere Gruppen im Rahmen der BABELUGA-Gruppeneinteilung verknüpft. Als zusätzlicher Parameter wurde in der vorliegenden Studie jedoch außerdem das Patientenalter erfasst, dagegen erfolgte keine bzw. nur eine geringgrade Erfassung von Leidensdruck und Veränderungsmotivation.

In Bezug auf das Therapiekonzept selbst waren in der vorliegenden Studie Patienten mit einer höheren Anzahl an beteiligten Disziplinen (Arzt, Ernährung, Psychologie) kurzfristig und einer längeren Therapiedauer insgesamt erfolgreicher. Im Umkehrschluss scheint eine Steigerung der beteiligten Disziplinen sowie eine Verlängerung der Therapiedauer bei einer zunehmenden Anzahl vorliegender Negativprädiktoren sinnvoll. Betrachtet man auch an dieser Stelle das Vorgehen nach der BABELUGA-Methode, so ist die oben genannte Einteilung der Patienten in Gruppen von A bis D anhand ihrer Voraussetzungen darin begründet, dass auch für jede dieser Gruppen ein eigenes Therapiekonzept mit Zunahme der beteiligten Modalitäten und der 
Behandlungsdauer besteht. Ernst et al. verwenden in diesem Zusammenhang den Begriff einer „Behandlungskette“ (Ernst und Wiegand 2010). Damit ist gemeint, dass verschiedene standardisierte Therapiemodule entsprechend den Gliedern einer Kette nebeneinander oder hintereinander gereiht werden können, damit also in verschiedenen Disziplinen gleichzeitig oder aufeinander folgend therapiert werden kann (für eine genaue Darstellung vgl. Seite 15). Eine ähnliche Strategie empfiehlt auch die KgAS (vgl. Abbildung 45) (Stachow et al. 2004).

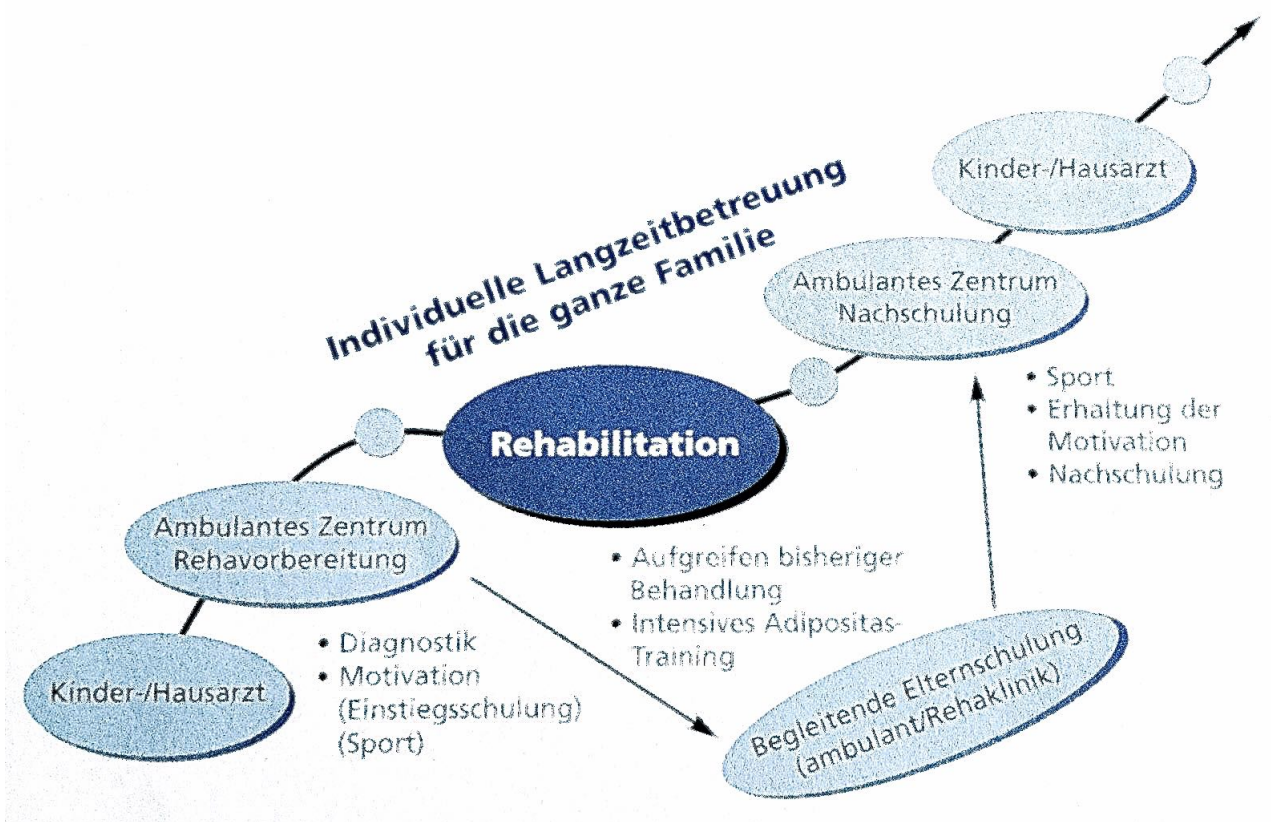

Abbildung 45: Behandlungskette für eine interdisziplinäre Adipositastherapie im Kindes- und Jugendalter entsprechend den Empfehlungen der KgAS (aus: Stachow et al. 2004)

Ein solches individualisiertes Vorgehen empfiehlt sich auch anhand der Ergebnisse der vorliegenden Untersuchung: Auch hier erscheint eine Anpassung der Therapiemodalität an die jeweils unterschiedlichen Therapievoraussetzungen und Problematiken der individuellen Situation adäquat.

Einige der genannten Faktoren sollten dabei bereits im Vorhinein evaluiert werden. Hierzu zählt eine Abklärung der Zielvorstellungen der Patienten. So waren z. B. in der vorliegenden Arbeit überhöhte und unrealistische Vorstellungen in Bezug auf die zu erwartende Gewichtsreduktion keine Seltenheit. Eine vorherige Erfassung solcher Vorstellungen und eine entsprechende Aufklärung des Patienten könnten einer Demotivation im Rahmen der Therapie vorbeugen. Weiterhin erhoffte sich nur ein geringer Teil der Patienten eine Abklärung von Komorbiditäten der Adipositas. Auch hier könnte vorab eine Evaluation hilfreich sein, um ggf. das Wissen um Bedeutung und Vermeidbarkeit von Begleiterkrankungen in den Fokus der Patienten zu rücken. Sowohl im Rahmen des BABELUGA- als auch des Obeldicks-Therapiekonzepts (Dobe, 2011) findet weiterhin im Vorhinein eine Beurteilung der Patientenmotivation statt. Bei zu geringer Motivationslage erfolgt im Rahmen des Obeldicks-Programms kein Therapieeinschluss, im Rahmen der BABELUGA-Methode wird dagegen vor Therapiebeginn der Versuch unternommen, die Patientenmotivation zu steigern. Letzteres Vorgehen erscheint sinnvoll, da auf diese Art nicht von vorherein ein Kind von der Behandlung ausgeschlossen wird. In der vorliegenden Studie könnte das Ziel einer Gewichtsreduktion im Gegensatz zu einer Stabilisierung sowie das Vorhandensein konkreter Zielgewichte im Gegensatz zum Fehlen solcher als Erfassung der Patientenmotivation aufgefasst werden. Es besteht jedoch aktuell noch keine einheitliche Meinung dazu, auf welche Weise der Motivationsgrad am besten überprüft bzw. gesteigert werden kann.

Weiterhin erscheint es von Vorteil, bereits im Vorhinein eine Abklärung psychischer Auffälligkeiten bzw. Erkrankungen vorzunehmen, da deren Vorliegen mit einer geringeren Gewichtsreduktion assoziiert ist. Eine beispielhafte Möglichkeit hierzu ist das Ausfüllen eines 
SDQ-Fragebogens vor Therapieplanung. Bei etwaigen Auffälligkeiten könnte eine weitere Abklärung durch einen Kinder- und Jugendpsychiater erfolgen. Sollte sich das Vorliegen psychischer Komorbiditäten bestätigen, kann eine Kinder- und Jugendpsychotherapie oder eine kinder- und jugendpsychiatrische Mitbehandlung in die Adipositastherapie integriert werden.

Daneben gewinnen auch die von der AGA empfohlenen Therapiebausteine einer Adipositastherapie im Kindes- und Jugendalter („Medizin“, „Ernährung“, „Bewegung“, „Verhaltenstherapie“, „Elternschulung“) im Hinblick auf die Ergebnisse der vorliegenden Untersuchung abhängig von der jeweiligen Situation an Bedeutung (Wabitsch und Kunze 2015). So fällt offenkundig dem Baustein „Medizin“ beim Vorliegen von Komorbiditäten ein größeres Gewicht zu. Das Einführen einer sportlichen Aktivität scheint insbesondere für Kinder und Jugendliche von Vorteil, die nicht ohnehin einer sportlichen Betätigung (insbesondere im Verein) nachgehen. Gerade eine sportliche Betätigung in extern vorgegebener Häufigkeit und Dauer erscheint im Hinblick auf die Ergebnisse der vorliegenden Arbeit empfehlenswert, beispielsweise auch in Form einer Adipositassportgruppe. Der Vorteil einer solchen Gruppe ist ein Anheben der Hemmschwelle durch die gemeinsame Aktivität mit anderen adipösen Kindern und Jugendlichen sowie ein Fehlen von Leistungsorientiertheit, welche mit einer Demotivation einhergehen kann (McWhorter et al. 2003). Einer Elternschulung bzw. Familieneinbindung muss ein größeres Gewicht zufallen, wenn nur geringe elterliche Ressourcen vorhanden sind. Anhand der Ergebnisse der vorliegenden Studie ist dies zum einen bei elterlicher Adipositas anzunehmen. Da neben genetischen Komponenten auch eine Übertragung elterlicher Gewohnheiten auf ihre Kinder angenommen wird, kann von einem durchschnittlich geringeren elterlichen Handlungswissen bzw. geringerer elterlicher Handlungsmotivation bezüglich adipogener Verhaltensweisen bei adipösen Eltern ausgegangen werden. Zum anderen scheint auch ein niedriger SES mit einem geringeren Therapieerfolg einherzugehen. Auch an dieser Stelle kann von geringeren Ressourcen von Patient und Familie ausgegangen werden. Um dieser Situation zu begegnen kann neben einer intensivierten Elterneinbindung in die Therapie auch eine Unterstützung durch das Jugendamt, z. B. mit Einsatz eines Familienhelfers, in Betracht gezogen werden. Gegebenenfalls kann auch ein stationärer Aufenthalt mit vorübergehender Ausschaltung eines obesigenen destruktiven Umfelds erwogen werden.

Insgesamt wird durch eine solche Individualisierung der Therapie neben dem Einsatz der bewährten Therapiebausteine „Medizin“, „Ernährung“, „Bewegung“, „Verhaltenstherapie“ und „Elternschulung“ auch ein Fokus auf die psychosozialen Ressourcen gelegt. Durch Versuche eines Ressourcenaufbaus bzw. einer Motivationssteigerung, gegebenenfalls auch einer Psychotherapie und einer sozialen Unterstützung durch das Jugendamt kann es Patienten und ihren Familien gezielter möglich gemacht werden, von Therapieangeboten $\mathrm{zu}$ profitieren (Wiegand et al. 2005). Eine Steuerung der verschiedenen Behandlungsmodule kann beispielsweise durch den Haus- oder Kinderarzt erfolgen. Wiegand et al. betonen außerdem, dass ein solches Therapiekonzept nur langfristig wirksam sein kann. Wie bereits in der Einleitung angesprochen, wird Adipositas im Kindes- und Jugendalter zunehmend als chronische Erkrankung wahrgenommen (Disse und Zimmer 2014). Studienergebnisse, nach denen höhere Therapiedauern oder Erhaltungsprogramme nach Therapieende mit besseren Therapieerfolgen einhergehen, stützen diese Klassifizierung (Deforche et al. 2005; Wilfley et al. 2007). Damit erscheint eine langfristige Planung von Therapieangeboten oder zumindest ein Anschluss von speziellen Erhaltungsprogrammen sinnvoll.

Ein Vorschlag für die Modifikation einer Adipositastherapie kann naturgemäß nicht anhand einiger weniger Untersuchungen festgemacht werden. Um die Effektivität der besprochenen Vorgehensweisen zu bestätigen, sind folglich zukünftig weitere Studien nötig. Um auf Einflussfaktoren präziser reagieren zu können, müssen dabei auch weiterhin die Prädiktoren für eine erfolgreiche Adipositastherapie im Kindes- und Jugendalter Gegenstand der Forschung sein. Insgesamt ist jedoch eine „maßgeschneiderte“ Adipositastherapie momentan ein vielversprechender Ansatz zur Optimierung der bisher eher limitierten Therapieerfolge. 


\section{$5 \quad$ Zusammenfassung}

Die Erfolge in der Adipositastherapie von Kindern und Jugendlichen sind aktuell insgesamt eher begrenzt. Es stellt sich daher die Frage, wie man heutige Therapieangebote noch optimieren kann. In diesem Kontext kommt der Erfassung von Prädiktoren, die den Erfolg positiv oder aber negativ beeinflussen können, eine zentrale Bedeutung zu. Dies gilt nicht nur für den unmittelbaren Therapieerfolg sondern insbesondere auch für langfristige Ergebnisse noch Jahre nach der Behandlung.

$\mathrm{Zu}$ diesem Zweck erfolgte in der vorliegenden Kohortenstudie ein Einschluss von 358 Kindern und Jugendlichen, die zwischen dem 01.10.2004 und dem 30.09.2013 eine Adipositastherapie im Sozialpädiatrischen Zentrum der Universitätsmedizin Göttingen erhalten haben. Neben Gewichts- und Größendaten zu Therapiebeginn ( $\left.\mathrm{T}_{0}\right)$, Therapieende $\left(\mathrm{T}_{1}\right)$ und zu einem Followup-Zeitpunkt ( $T_{2}$, frühestens ein Jahr nach Beendigung der Therapie) wurden für diese Patienten verschiedene Parameter erfasst. Die Gewichtsentwicklung wurde in Form der BMI-SDSReduktion für drei verschiedene Zeiträume erhoben: Kurzfristig von Therapiebeginn bis -ende $\left(\mathrm{T}_{0}-\mathrm{T}_{1}\right)$, langfristig vom Therapieende bis zum Follow-up-Zeitpunkt $\left(\mathrm{T}_{1}-\mathrm{T}_{2}\right)$ sowie für den gesamten Verlauf $\left(\mathrm{T}_{0}-\mathrm{T}_{2}\right)$. Es erfolgte eine Untersuchung auf Zusammenhänge zwischen der Gewichtsentwicklung über die verschiedenen Zeiträume sowie den unterschiedlichen erfassten möglichen Einflussgrößen.

Von den eingeschlossenen Kindern und Jugendlichen waren 192 männlich und 166 weiblich, das durchschnittliche Alter bei Therapiebeginn lag bei 13,6 Jahren (KI: \pm 3,5). Die durchschnittliche Therapiedauer betrug 12,1 Monate (KI: $\pm 23,4$ ). In die Follow-up-Erhebung gingen Daten von 149 Patienten ein. Die durchschnittliche postinterventionelle Zeit umfasste 4,1 Jahre (KI: $\pm 4,8)$. Im Schnitt lag die kurzfristige erreichte BMI-SDS-Reduktion bei 0,07 (KI: $\pm 0,08$ ), langfristig wurde ein Wert von 0,47 (KI: $\pm 1,36$ ) und insgesamt von 0,56 (KI: $\pm 0,56$ ) erreicht (entsprechend den Ergebnissen einer pP-Analyse).

Es konnten verschiedene Parameter mit Assoziation zur BMI-SDS-Reduktion der eingeschlossenen Patienten erfasst werden: Grundsätzlich konnten Patienten, die über den Therapiezeitraum hohe BMI-SDS-Reduktionen erzielten, auch über den gesamten Verlauf höhere Reduktionen verzeichnen. Damit stellt sich in der vorliegenden Studie der kurzfristige Therapieerfolg selbst als wichtiger Prädiktor für den langfristigen Verlauf dar. Zudem waren verschiedene Patienten-Charakteristika mit signifikant höheren Reduktionen assoziiert: Hierzu zählten ein niedrigerer BMI-SDS zu Therapiebeginn sowie das Vorliegen konkreter Vorstellungen bezüglich des angestrebten Zielgewichts. In Bezug auf das Alter zum Zeitpunkt des Therapiebeginn zeigten sich kurzfristig jüngere Patienten erfolgreicher, während auf lange Sicht ältere Patienten höhere Reduktionen erzielten. Als wichtiger, auch im Rahmen der Therapie beeinflussbarer Faktor, präsentierte sich die körperliche Aktivität: So war eine Steigerung der körperlichen Aktivität über den Therapiezeitraum auch mit einer signifikant höheren BMI-SDS-Reduktion über diese Zeit verknüpft. Auch generell erreichten Patienten, die einen Vereinssport ausübten, signifikant höhere Reduktionen. Weiterhin gingen positivere Bewertungen der Therapie mit höheren Reduktionen einher. Schließlich waren auch intensivere Therapiekonzepte mit einer höheren Zahl an eingesetzten Modalitäten ( $d . h$. Ernährungsschulung, psychologische und ärztliche Betreuung) sowie einer längeren Therapiedauer mit höheren Reduktionen assoziiert, wenngleich hierbei die erreichten p-Werte oberhalb des Signifikanzniveaus von $\mathrm{p}=0,05$ angesiedelt waren.

Dagegen erreichten im Rahmen einer Analyse des sozioökonomischen Status Patienten ohne abgeschlossene Berufsausbildung nur geringere BMI-SDS-Reduktionen. Gleiches galt für Patienten, die sich neben der Adipositastherapie in zusätzlicher kinder- und jugendpsychiatrischer Behandlung befanden. Für den Parameter „Medienkonsum“ sowie den Migrationsstatus konnte keinerlei Zusammenhang zur BMI-SDS-Reduktion erhoben werden.

Das Wissen um die Faktoren, die einen positiven bzw. negativen Einfluss auf die Gewichtsreduktion nehmen, eröffnet neue Möglichkeiten in der Behandlung von übergewichtigen und adipösen Kindern und Jugendlichen. So kann durch dieses Wissen eine 
gezielte Anpassung und Individualisierung der Therapie erfolgen: Eine Ausrichtung der beteiligten Professionen an den jeweiligen Voraussetzungen des Patienten mit begleitender Intensivierung der Therapie bei Vorliegen von Negativprädiktoren wird möglich. Um dieses Konzept zu stützen, sind gleichwohl zukünftige Studien mit Erfassung des tatsächlichen Erfolgs solcher „maßgeschneiderter" Behandlungen nötig. Insgesamt handelt es sich jedoch um einen vielversprechenden Ansatz zur Optimierung heutiger Therapieangebote. 


\section{Anhang}

Patientenfragebogen zum Zweck der Follow-Up-Datenerhebung:

Frau/ Herr

\section{I) Anmeldung und Erwartungen}

Sie haben erstmalig am

mit Ihrer Tochter/ Ihrem Sohn aufgesucht.

(Name) das SPZ wegen Ihres/ Seines Gewichtsproblems

1. Wie haben Sie von dieser Sprechstunde erfahren?
a. aus der Kinderklinik
b. vom Kinderarzt
c. durch Freunde/ Verwandte
d. aus dem Kindergarten/ der Schule
e. aus den Medien

2. Von wem ging die Motivation aus?

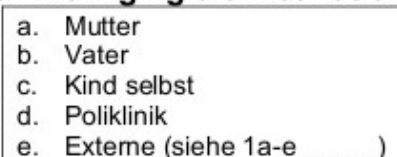

3. Hatten Sie eine Vorstellung davon, was Sie in der Sprechstunde erwartet?

a. Nein

b. Ja

4. Wenn ja, was haben Sie sich davon versprochen?
a. Abklärung einer medizinischen Grund-bzw. Folgeerkrankung
b. Abklärung anderer Ursachen
c. Ernährungsschulung
d Psychologische Unterstützung
e. Einzeltherapie
f. Gruppentherapie
g. Kuraufenthalt

h. Hatten Sie ein bestimmtest Ziel vor Augen?
a. Gewicht abnehmen
b. Gewicht halten
c. Kein bestimmtes Ziel

\section{II) Beurteilung der SPZ-Arbeit}

1.Wie zufrieden waren Sie mit der Arbeit des SPZ bzw. den einzelnen Therapeuten?

\begin{tabular}{|l|l|l|l|l|l|}
\hline & $\begin{array}{l}1 \\
\text { (sehr zufrieden) }\end{array}$ & 2 & 3 & 4 & 5 \\
Anmeldung/Terminmitteilung & & & & & \\
\hline Wartezeit & & & & & \\
\hline Arzt & & & & & \\
\hline Ernährungsberatung & & & & & \\
\hline Psychologie & & & & & \\
\hline
\end{tabular}


2. Haben Sie von der ärztlichen Betreuung profitiert?

\begin{tabular}{|l|l|l|l|l|}
\hline $\mathbf{1}$ (sehr) & $\mathbf{2}$ & $\mathbf{3}$ & $\mathbf{4}$ & $\mathbf{5}$ (gar nicht) \\
\hline
\end{tabular}

Was hat Ihnen besonders gefallen?

Was hat lhnen nicht gefallen?

3. Haben Sie von der Ernährungsberatung profitiert?

\begin{tabular}{|l|l|l|l|l|}
\hline $\mathbf{1}$ (sehr) & 2 & 3 & 4 & (gar nicht) \\
\hline
\end{tabular}

Was hat Ihnen besonders gefallen?

Was hat Ihnen nicht gefallen?

4. Haben Sie von der psychologischen Unterstützung profitiert?

\begin{tabular}{|l|l|l|l|l|}
\hline $\mathbf{1}$ (sehr) & $\mathbf{2}$ & $\mathbf{3}$ & $\mathbf{4}$ & $\mathbf{5}$ (gar nicht) \\
\hline
\end{tabular}

Was hat Ihnen besonders gefallen?

Was hat Ihnen nicht gefallen?

\section{III) Umsetzung der Therapievorschläge und aktuelles Verhalten}

1. Hat sich das Freizeitverhalten ihres Kindes nach dem Besuch im SPZ verändert?

\begin{tabular}{|l|l|l|l|}
\hline & öfter & gleich & weniger \\
\hline Medienkonsum & & & \\
\hline Körperliche & & & \\
Aktivtät & & & \\
\hline Sport & & & \\
\hline Freunde treffen & & & \\
\hline Familienaktivitäten & & & \\
\hline
\end{tabular}

2. Ist sie/ er Mitglied im Sportverein

\begin{tabular}{|c|c|c|}
\hline a. & $\begin{array}{l}\text { Ja (Sport: } \\
\text { Monat) } \\
\text { Nein }\end{array}$ & $L^{x /}$ \\
\hline & $\begin{array}{l}\text { Gibt es eine andere regelmäßige sportliche Betätigung? } \\
\text { ( }\end{array}$ & \\
\hline
\end{tabular}

3. Wiegt sich regelmäßig?

\begin{tabular}{|l|l|l|l|}
\hline Nein & täglich & wöchentlich & monatlich \\
\hline
\end{tabular}


4. Wissen Sie das aktuelle Gewicht und die Größe von

(Name)?

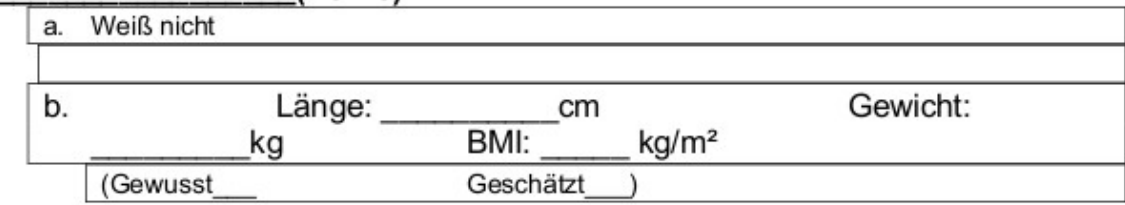

Sie sind seit mehr als einem halben Jahr nicht mehr bei uns gewesen.

5. Warum haben Sie die Therapie beendet?

\begin{tabular}{|ll|}
\hline a. & Therapie-Erfolg \\
\hline b. & Wiedervorstellungstermin vergessen \\
\hline c. & Anfahrt zu weit \\
\hline d. & Lange Wartezeit \\
\hline e. & Mangelnder Erfolg \\
\hline f. & Zeitmangel der Eltern \\
\hline g. & Zeitmangel der Kinder \\
\hline h. & Mein Kind wollte nicht mehr weitermachen \\
\hline i. & Umsetzung zu schwierig \\
\hline j. & Wir sind mit den Ärzten/ Therapeuten nicht klargekommen \\
\hline k. & Sonstiges \\
\hline
\end{tabular}

(Kompositionelle Frage, Mehrfachantwort möglich)

6. Würden Sie erneut ein Unterstützungsangebot des Sozialpädiatrischen Zentrums nutzen wollen?

\begin{tabular}{|l|l}
\hline Ja & Nein
\end{tabular}

\section{Fragen zu den allgemeinen Lebensbedingungen}

\section{Bei wem lebt das Kind?}

\begin{tabular}{|l|}
\hline a) Eltern \\
\hline b) Vater \\
\hline c) Mutter \\
\hline d) Patchwork-Familie \\
\hline d) Pflegeeltern \\
\hline e) Einrichtung \\
\hline f) ohne Eltern \\
\hline
\end{tabular}

\section{Welche Schule/Ausbildung/Beruf geht ihr Kind nach?}

\begin{tabular}{|l|}
\hline Kindergarten \\
\hline Grundschule \\
\hline Hauptschule \\
\hline Realschule \\
\hline Regelschule \\
\hline Gesamtschule \\
\hline Gymnasium \\
\hline Schulabschluß: \\
Studium/Berufsausbildung: \\
\hline
\end{tabular}


Ausgeübter Beruf:

3. Ausbildung/Beruf der Eltern
Mutter:
Schulabschluss:
Berufsausbildung:
Jetzige Berufstätigkeit:
Wochenarbeitszeit:
Vater:
Schulabschluss:
Berufsausbildung:
Jetzige Berufstätigkeit:
Wochenarbeitszeit:

Wissen Sie das aktuelle Gewicht und die Größe von Ihnen und dem anderen Elternteil?

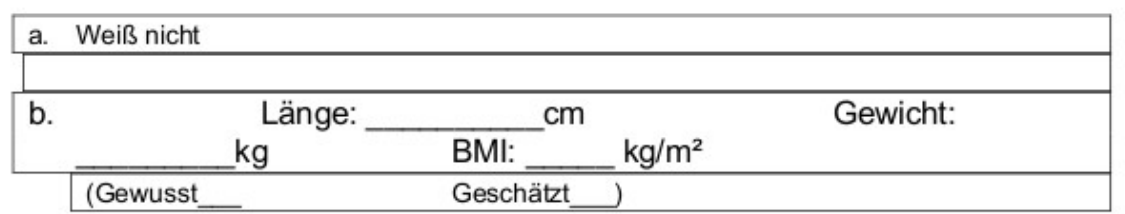

\section{Kontaktfragen:}

\begin{tabular}{|ll|}
\hline c. & Anzahl der Kontaktversuche: \\
d. & Dauer des Interviews: \\
\hline e. & Einschätzung des Antwortverhaltens: \\
& $1-5:$ \\
\hline
\end{tabular}


Patienteninformations- und Einwilligungsbögen:

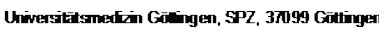

Patienteninformation

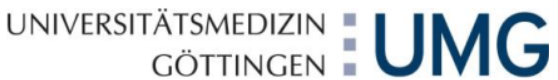

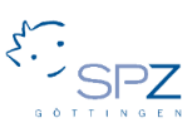

Klinik für Kinder- und Jugendmedizin Direktain: Prof. Dr. med. Jutta Gärtner

Sozialpädiatrisches Zentrum Ât diche Leitung: Prof. Dr. med Krut Brockmamn

37099 Götingen Brietpost Rotest Koch Straße 40, 37075 Gätingen Adresse
0561 / 39-10358 Teteton Setretariat 0xk1 / 39-10368 Telefon Selvetariat 0561 / 3913241 Teletion Annethung
OE51 / 39 13245 Fa makas roetimed uri-goetingen de $E$-and 10.08 .2016 Dr. Rö OE561 / 3913241

Studie " Charakterisierung von positiven und negativen Prādiktoren für eine erfolgreiche AdipositasBehandlung nach Abschluss der Behandlung."

Liebe Eltem,

Wie Sie sehr wahrscheinlich wissen, ist Adipositas („krankhaftes Ūbergewicht) eine Erkrankung, die in unserer Bevōlkerung stetig zunimmt. Sie haben ihr Kind in unserer Klinik zur Diagnostik und/oder Therapie vorgestellt. Ziel unserer Schwerpunktsprechstunde zur Adipositas im Sozialpādiatrischen Zentrum ist es, durch ein umfangreiches Konzept, das neben der AbkJārung organischer Ursachen auch psychosoziale Aspekte berücksichtigt, betroffenen Kinder und Jugendlichen zu helfen. Mehr als 1000 Kinder und Jugendliche mit Adipositas, die aus Südniedersachsen, Nordhessen und Ostthüringen überwiesen wurden, haben wir bislang betreut. Leider ist der langfristige klinische Verlauf bei Kindem und Jugendlichen mit Adipositas bis heute nur ungenügend erforscht und hāufig sind die langfristigen Behandlungserfolge nicht befriedigend. Daher mōchten wir jetzt die Gründe, die langfristig zu einer erfolgreichen oder nicht erfolgreichen Ānderung der Lebensstilfaktoren Emāhrung und Bewegung führen, untersuchen.

Wir mōchten Sie daher bitten an einer Befragung teilzunehmen und uns Auskunft über den weiteren Verlauf der Erkrankung ihres Kindes zu geben. In einem telefonischen Gesprāch werden wir Ihnen verschiedene Fragen stellen. Der dabei verwendete Fragebogen liegt zu Ihrer Ansicht diesem Schreiben bei. Sie tragen mit Ihrer Telnahme dazu bei, dass wir die Behandlung des Ūbergewichtes bei Kindem und Jugendlichen weiter verbessem kōnnen. Die Daten, die wir erheben, werden anonym isiert gespeichert und dienen lediglich der internen Auswertung und Verbesserung unserer Therapieangebote. Sollten Sie zu unserer Studie weitere Fragen haben, kōnnen Sie sich geme telefonisch oder per Emal an uns wenden. Bitte senden sie uns die Patienteneinverstāndniserklārung in dem vorfrankierten Briefumschlag in den nāchsten 14 Tagen zurück.

Mit freundlichen Grüßen,
Prof. Dr. J. Gārtner
Prof. Dr. K. Brockmann
Dr. M. Lingen
Dr. M. Rōbl 


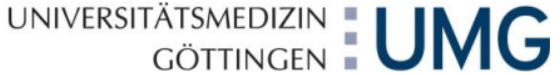

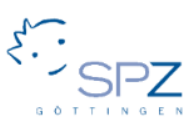

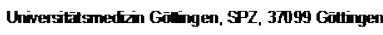

Patienteninformation (12-16 Jahre)
Klinik für Kinder- und Jugendmedizin Direktain: Prof. Dr. med. Jutta Gärtner

\section{Sozialpädiatrisches Zentrum}

Ärdliche Leitung Prof. Dr. med Knut Brockmann

37009 Göttingen Brietpost

Rowet-Knch-Strake 40, 37075 Gatingen Adresse

D551 / 39-10348 Teleton Selvetariat

D651 / 3913241 Telefion Amnetdrng

D551 / 39-13245 Fax

makus. methomed uni goetingen.de E-m:-

10.08 .2016

Dr. Rṓt

D551 / 3913241

Studie

"Telefon Case-Management"Angebot zur Verbesserung der Therapie-Compliance und des Therapie-Erfolges adipōser Kinder und Jugendlicher

Liebe / $r$

wir mōchten Dich fragen, ob Du bereit bist, an der nachfolgend beschriebenen Studie telzunehmen. Die Teilnahme an der Studie ist natürlich freiwillig.

Studien dienen der Forschung in der Medizin. Ob Du bei der Studie mitmachen mōchtest, kannst Du zusammen mit Deinen Eltern selbst entscheiden. Deine Telnahme ist, auch wenn Deine Eltem zustimmen, freiwillig; keiner zwingt Dich. Wenn Du nicht an der Studie teinehmen oder spāter aus ihr ausscheiden mōchtest, entstehen Dir daraus keine Nachteile. Ob Du mitmachen willst, musst Du nicht gleich entscheiden; lass Dir ruhig Zeit, um darüber nachzudenken. Diese Information soll Dir bei Deiner Entscheidung helfen, denn es ist wichtig, dass Du alles verstehst Auch Deine Eltem haben von uns eine schriftiche Information bekommen. Wenn Ihr alles gelesen habt, werdet Ihr Euch darüber unterhalten. Deine Eltern werden sicher die meisten Fragen, die Du hast, beantworten kōnnen. Wenn Du noch mehr über die Studie wissen mōchtest, frage uns, wir werden Dir alles erklären.

Warum wird die Studie durchgeführt?

Du bist mit Deinen Eltem in unsere Sprechstunde für übergewichtige Kinder gekommen. Ūbergewicht bedeutet, dass das Kōrpergewicht bezogen auf die Kōrpergrōße zu hoch ist. Sehr selten kann als Ursache des Ūbergewichtes eine Erkrankung gefunden werden. Viel hāufiger stimmt jedoch in Gleichgewicht zwischen der Nahrungszufuhr und dem Energieverbrauch des Kōrpers etwas nicht. Deine Eltern sind zum einen mit Dir in unsere Sprechstunde gekommen, damit wir krankhafte Ursachen des Übergewichtes ausschließen und zum anderen wünschen Sie sich Unterstützung und Tipps, wie es mōglich ist, dass Dein Kōrpergewicht zu Deiner Kōrpergrōße wieder passt. Es ist sehr wichtig, dass die Nahrungszufuhr und der Energieverbrauch wieder ins Gleichgewicht kommen, damit die Gefahr, dass man spāter andere Erkrankungen wie Z. B. Zuckerkrankheit, Leberverfettung, Galensteinleiden oder Bluthochdruck, aufgrund des Ūbergewichtes bekommt, vermindert werden kann. In der Sprechstunde für übergewichtige Kinder und Jugendliche hilft Dir und Deinen Eltem ein Team von Ārzten, Emāhrungsberaterinnen und Psychologen. Wenn eine krankhafte Ursache des Ūbergewichtes ausgeschlossen werden konnte, ist die Therapie des Ūbergewichtes eine Verānderung der Emāhrung und von Bewegung. Das bedeutet, dass die Nahrungsmenge für Dich optimal angepasst werden muss und dass deine Aktivitāt gesteigert werden sollte. Leider ist die Verānderung der Nahrungsaufnahme und des Energieverbrauchs in Altag hāufig sehr schwierig. Daher bieten wir Dir und Deinen Eltern mit dieser Studie ein zusātzliches Angebot zu den ambulanten Terminen in unserer Klinik. Das 


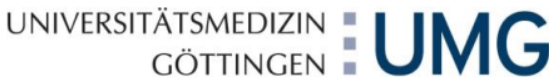

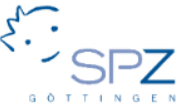

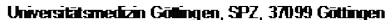

Patienteninformation (6-11 Jahre)
Klinik für Kinder- und Jugendmedizin Direktain: Prof. Dr. med. Jutta Gärner

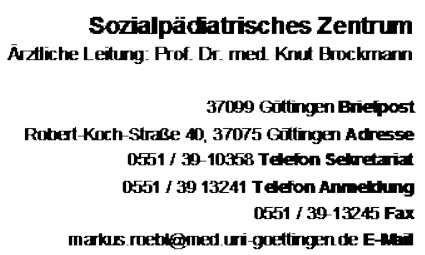

\subsubsection{6} Dr. Rän $0651 / 3913241$

Studie

Charakterisierung von positiven und negativen Prädiktoren für eine erfolgreiche Adipositas-

Behandlung nach Abschluss der Behandlung.

Liebe / $\mathbf{r}$

Wr mōchten Dich und Deine Eltem um die Teinahme an unserer kFischen Studie bätten.

Studien dienen der Forschung in der Medizin.

Du bist mit Deinen Eltern in unsere Sprechstunde für übergewichtige Kinder gekommen. Ūbergewicht bedeutet, dass das Körpergewicht bezogen auf die Körpergröße zu hoch ist. Sehr selten kann als Ursache des Ūbergewichtes eine Erkrankung gefunden werden. Viel häufiger stimmt jedoch im Gleichgewicht zwischen der Nahrungsaufhahme und dem Energieverbrauch des Kōrpers etwas nicht. Deine Eltern sind zum einen mit $D^{-}$i in unsere Sprechstunde gekommen, damit wi krankhafte Ursachen des Ūbergewichtes ausschließen und zum anderen wünschen Sie sich Unterstützung und Tipps, wie es mōglich ist, dass Dein Kōrpergewicht wieder zu Deiner Kōrpergrōße passt. Es ist sehr wichtig, dass Nahrungszufuhr und der Energieverbrauch wieder ins Geichgewicht kommen, damit die Gefahr, dass man spāter andere Erkrankungen aufgrund des Ūbergewichtes bekommt, vermindert werden kann. In der Sprechstunde für übergewichtige Kinder hat Dich und Deinen Btem ein Team von Ärzten, Emāhrungsberaterinnen und Psychologen betreut. Manchen Familien fält es leichter, die Nahrungszufuhr und den Energieverbrauch wieder ins Geichgewicht zu bringen als anderen Familien. Nachdem nach Deinem letzen Vorstelhngstermin in unserer Adpositas-Sprechstunde mehr als 6 Monate vergangen sind, mōchten wir Deine Etem bïtten, uns telefonisch ein paar Fragen zum Verlauf deines Gewichtes und der Begleitumstānde zu beantworten. Anhand der ehaltenen Informationen mōchten besser verstehen, warum es einigen Familen gelingt das Gleichgewicht von Nahrungszufuhr und Energieverbrauch herzustelen und anderen Famile nicht. Mì diesen Informationen mōchten wir das Therapie-Angebơt für übergewichtige Kinder in unserem Sozialpādiatrischen Zentrum verbessern.

AN WEN KANNST DU NOCH FRAGEN STELLEN?

Wenn Du weitere Fragen hast, kannst Du Dich geme an uns wenden. Wir beentworten alle Deine Fragen sehr gerne Mit freundichen Grüßen,
Prof. Dr. J. Gärner
Prof. Dr. K Brockmann
D. M. Lingen
Dr. M. Rōb 


\section{$7 \quad$ Literaturverzeichnis}

Accurso EC, Norman GJ, Crow SJ, Rock CL, Boutelle KN (2014): The role of motivation in family-based guided self-help treatment for pediatric obesity. Child Obes 10, 392-399

Alexy U, Reinehr T, Sichert-Hellert W, Wollenhaupt A, Kersting M, Andler W (2006): Positive changes of dietary habits after an outpatient training program for overweight children. Nutr Res $\underline{26}, 202-208$

Alexy U, Clausen K, Kersting M (2008): Die Ernährung gesunder Kinder und Jugendlicher nach dem Konzept der Optimierten Mischkost. Ernährungs Umschau 08, 168-177

Alviola PAt, Nayga RM, Jr., Thomsen MR, Danforth D, Smartt J (2014): The effect of fast-food restaurants on childhood obesity: a school level analysis. Econ Hum Biol $\underline{12}$, 110-119

Andersen RE, Crespo CJ, Bartlett SJ, Cheskin LJ, Pratt M (1998): Relationship of physical activity and television watching with body weight and level of fatness among children: results from the Third National Health and Nutrition Examination. Survey Jama 279, 938-942

Anderson P, Butcher K (2006): Childhood obesity: trends and potential causes. Future Child 16, 19-45

Anderson SE, Must A (2005): Interpreting the continued decline in the average age at menarche: results from two nationally representative surveys of U.S. girls studied 10 years apart. J Pediatr $\underline{147}$, 753760

Angrisani L, Favretti F, Furbetta F, Paganelli M, Basso N, Doldi SB, Iuppa A, Lucchese M, Lattuada E, Lesti G, et al. (2005): Obese teenagers treated by Lap-Band System: the Italian experience Surgery. 138 , 877-881

Atlantis E, Barnes EH, Singh MA (2006): Efficacy of exercise for treating overweight in children and adolescents: a systematic review. Int J Obes $\underline{30}$, 1027-1040

Baker JL, Olsen LW, Sorensen TI (2008): Childhood body mass index and the risk of coronary heart disease in adulthood. Ugeskr Laeger 170, 2434-2437

Barlow SE (2007): Expert committee recommendations regarding the prevention, assessment, and treatment of child and adolescent overweight and obesity: summary report. Pediatrics 120 Suppl 4, S164-192

Barlow SE, Dietz WH (1998): Obesity evaluation and treatment: Expert Committee recommendations. The Maternal and Child Health Bureau, Health Resources and Services Administration and the Department of Health and Human Services. Pediatrics 102, E29

Barlow SE, Ohlemeyer CL (2006): Parent reasons for nonreturn to a pediatric weight management program. Clin Pediatr 45 , 355-360

Biehl A, Hovengen R, Grøholt E-K, Hjelmesæth J, Strand BH, Meyer HE (2014): Parental marital status and childhood overweight and obesity in Norway: a nationally representative cross-sectional study. BMJ open $\underline{4}$ e 004502

Blossfeld HP, Kiernan K: The new role of women: family formation in modern societies. Westview Press, Boulder 1995

Böhler T, Goldapp C, Mann R, Reinehr T, Bullinger M, Holl R, Hoffmeister U, van Egmond-Frohlich A, Ravens-Sieberer U, Wille N, et al. (2013): Sensitivity analysis of weight reduction results of an observational cohort study in overweight and obese children and adolescents in Germany: the evakuj study. Pediatr Rep $\underline{5}$, e16

Bosch J, Stradmeijer M, Seidell J (2004): Psychosocial characteristics of obese children/youngsters and their families: implications for preventive and curative interventions. Patient Educ Couns 55, 353362

Braet C (2006): Patient Characteristics as Predictors of Weight Loss after an Obesity Treatment for Children. Obesity 14, 148-155

Brettschneider AK, Schaffrath Rosario A, Kuhnert R, Schmidt S, Wiegand S, Ellert U, Kurth BM (2015): Updated prevalence rates of overweight and obesity in 11- to 17-year-old adolescents in Germany. Results from the telephone-based KiGGS Wave 1 after correction for bias in selfreports. BMC Public Health $\underline{15}, 1101$

Burniat W, Cole TJ, Lissau I, Poskitt EM: Child and adolescent obesity: Causes and consequences, prevention and management. Cambridge University Press, Cambridge 2006

Byrne S, Cooper Z, Fairburn C (2003): Weight maintenance and relapse in obesity: a qualitative study. International journal of obesity $\underline{27}, 955-962$ 
Caroli M, Burniat W: Dietary Management. In: Burniat W, Cole TJ, Lissau I, Poskitt EM (Hrsg.): Child and Adolescent Obesity. Causes and Consequences; Prevention and Management. Cambridge University Press, Camebridge 2006

Caroli M, Argentieri L, Cardone M, Masi A (2004): Role of television in childhood obesity prevention. Int J Obes Relat Metab Disord 28 Suppl 3, S104-108

Chan GM (1992): Performance of dual-energy x-ray absorptiometry in evaluating bone, lean body mass, and fat in pediatric subjects. J Bone Miner Res $\underline{7}, 369-374$

Chen AY, Escarce JJ (2010): Family structure and childhood obesity, early childhood longitudinal studykindergarten cohort. Prev Chronic Dis $\underline{7}$, A50

Civantos Modino S, Guijarro de Armas MG, Monereo Mejias S, Montano Martinez JM, Iglesias Bolanos P, Merino Viveros M, Ladero Quesada JM (2012): Hyperuricemia and metabolic syndrome in children with overweight and obesity. Endocrinol Nutr 59, 533-538

Cole TJ (1990): The LMS method for constructing normalized growth standards. Eur J Clin Nutr $\underline{44}$ 45-60

Cole TJ, Freeman JV, Preece MA (1995): Body mass index reference curves for the UK, 1990. Arch Dis Child 73, 25-29

Cole TJ, Bellizzi MC, Flegal KM, Dietz WH (2000): Establishing a standard definition for child overweight and obesity worldwide: international survey. Bmj $\underline{320}, 1240-1243$

Coon KA, Goldberg J, Rogers BL, Tucker KL (2001): Relationships between use of television during meals and children's food consumption patterns. Pediatrics 107, E7

Correll CU, Lencz T, Malhotra AK (2011): Antipsychotic drugs and obesity Trends. Mol Med 17, 97-107

Cousins JH, Rubovits DS, Dunn JK, Reeves RS, Ramirez AG, Foreyt JP (1992): Family versus individually oriented intervention for weight loss in Mexican American women. Public Health Rep 107, 549555

Craig L (2006): Does father care mean fathers share? A comparison of how mothers and fathers in intact families spend time with children. Gend Soc 20, 259-281

Crujeiras AB, Goyenechea E, Abete I, Lage M, Carreira MC, Martinez JA, Casanueva FF (2010): Weight regain after a diet-induced loss is predicted by higher baseline leptin and lower ghrelin plasma levels. J Clin Endocrinol Metab 95, 5037-5044

Daniels SR, Khoury PR, Morrison JA (1997): The utility of body mass index as a measure of body fatness in children and adolescents: differences by race and gender. Pediatrics $\underline{99}, 804-807$

de Onis M, Onyango AW, Borghi E, Siyam A, Nishida C, Siekmann J (2007): Development of a WHO growth reference for school-aged children and adolescents. Bull World Health Organ 85, 660-667

Deforche B, De Bourdeaudhuij I, Tanghe A, Debode P, Hills AP, Bouckaert J (2005): Post-treatment phone contact: a weight maintenance strategy in obese youngsters. Int J Obes 29, 543-546

Disse S, Zimmer K-P (2014): Adipositas bei Kindern ñ eine chronische Erkrankung? Dtsch Arztebl International $\underline{111}$ 816-817

Dobe M, Geisler A, Hoffmann D, Kleber M, von Köding P, Lass N, Müther S, Pohl B, Rose K, Schaefer A (2011): Das Obeldicks-Konzept. Bundesgesundheitsblatt-GesundheitsforschungGesundheitsschutz $\underline{54}$, 628-635

Ebbeling CB, Pawlak DB, Ludwig DS (2002): Childhood obesity: public-health crisis, common sense cure. Lancet $360,473-482$

Eberding A, Lemme M (2006): Adipositasschulung im Kindes- und Jugendalter. Familiärer Einfluss Elternaufgaben. Ernährungs Umschau $\underline{53}$, 25-29

Epstein LH, Wing RR (1987): Behavioral treatment of childhood obesity. Psychol Bull 101, 331-342

Epstein LH, Wing RR, Penner BC, Kress MJ (1985): Effect of diet and controlled exercise on weight loss in obese children. J Pediatr 107, 358-361

Epstein LH, Koeske R, Wing RR, Valoski A, Whitehead WE, Busch CM, Heller BR, Costa PT, Lau RR, Hartman KA (1988): The effect of family variables on child weight change. Health Psychol $\underline{5}, 1-11$

Epstein LH, McCurley J, Wing RR, Valoski A (1990): Five-year follow-up of family-based behavioral treatments for childhood obesity. J Consult Clin Psychol $\underline{58}$ 661-664

Epstein LH, Valoski A, Wing RR, McCurley J (1990): Ten-year follow-up of behavioral, family-based treatment for obese children. Jama 264, 2519-2523

Epstein LH, Valoski A, Wing RR, McCurley J (1994): Ten-year outcomes of behavioral family-based treatment for childhood obesity. Health Psychol $\underline{13}$, 373-383 
Epstein LH, Valoski AM, Vara LS, McCurley J, Wisniewski L, Kalarchian MA, Klein KR, Shrager LR (1995): Effects of decreasing sedentary behavior and increasing activity on weight change in obese children. Health Psychol 14, 109-115

Epstein LH, Gordy CC, Raynor HA, Beddome M, Kilanowski CK, Paluch R (2001): Increasing fruit and vegetable intake and decreasing fat and sugar intake in families at risk for childhood obesity. Obes Res 9 , 171-178

Ernst M, Wiegand S: Adipositastherapie bei Kindern und Jugendlichen einmal anders. Die BABELUGAMethode, Prävention Therapie, Selbstmanagement. Huber, Bern 2010

Fabricatore A, Wadden T, Womble L, Sarwer D, Berkowitz R, Foster GD, Brock J (2007): The role of patients' expectations and goals in the behavioral and pharmacological treatment of obesity. Int J Obes $\underline{31}, 1739-1745$

Fatima Y, Doi SA, Mamun AA (2015): Longitudinal impact of sleep on overweight and obesity in children and adolescents: a systematic review and bias-adjusted meta-analysis. Obes Rev 16, 137-149

Flechtner-Mors M, Thamm M, Wiegand S, Reinehr T, Schwab KO, Kiess W, Widhalm K, Holl RW (2012): Comorbidities related to BMI category in children and adolescents: German/Austrian/Swiss Obesity Register APV compared to the German KiGGS Study. Horm Res Paediatr 77, 19-26

Flores R (1995): Dance for health: improving fitness in African American and Hispanic adolescents. Public Health Rep $\underline{110}$ 189-193

Foster GD, Wadden TA, Vogt RA, Brewer G (1997): What is a reasonable weight loss? Patients' expectations and evaluations of obesity treatment outcomes. J Consult Clin Psychol $\underline{65}$, 79-85

French SA, Story M, Perry CL (1995): Self-esteem and obesity in children and adolescents: a literature review. Obes Res $\underline{3}$, 479-490

Fröhlich G, Pott W, Albayrak 0, Hebebrand J, Pauli-Pott U (2011): Conditions of long-term success in a lifestyle intervention for overweight and obese youths. Pediatrics $\underline{128}$, e779-785

Galan I, Gandarillas A, Febrel C, Meseguer C (2001): Validation of self-reported weight and height in an adolescent population. Gac Sanit 15, 490-497

Garcia-Morales LM, Berber A, Macias-Lara CC, Lucio-Ortiz C, Del-Rio-Navarro BE, Dorantes-Alvarez LM (2006): Use of sibutramine in obese mexican adolescents: a 6-month, randomized, double-blind, placebo-controlled, parallel-group trial. Clin Ther $\underline{28}$, 770-782

Gidding SS, Dennison BA, Birch LL, Daniels SR, Gillman MW, Lichtenstein AH, Rattay KT, Steinberger J, Stettler N, Van Horn L (2006): Dietary recommendations for children and adolescents: a guide for practitioners. Pediatrics $\underline{117}$, 544-559

Goran MI, Treuth MS (2001): Energy expenditure, physical activity, and obesity in children. Pediatr Clin North Am $\underline{48}, 931-953$

Gordon-Larsen P, McMurray RG, Popkin BM (2000): Determinants of adolescent physical activity and inactivity patterns. Pediatrics 105, E83

Gortmaker SL, Must A, Perrin JM, Sobol AM, Dietz WH (1993): Social and economic consequences of overweight in adolescence and young adulthood. N Engl J Med $\underline{329}$ 1008-1012

Goulding A, Taylor RW, Gold E, Lewis-Barned NJ (1996): Regional body fat distribution in relation to pubertal stage: a dual-energy X-ray absorptiometry study of New Zealand girls and young women. Am J Clin Nutr $\underline{64}, 546-551$

Guillaume M (1999): Defining obesity in childhood: current practice. Am J Clin Nutr $\underline{70}$, 126s-130s

Hansson LM, Karnehed N, Tynelius P, Rasmussen F (2009): Prejudice against obesity among 10-year-olds: a nationwide population-based study. Acta Paediatr 98, 1176-1182

Hauner H, Meier M, Wendland G, Kurscheid T, Lauterbach K (2004): Weight reduction by sibutramine in obese subjects in primary care medicine: the SAT Study. Exp Clin Endocrinol Diabetes 112, 201207

Hemmelmann C, Brose S, Vens M, Hebebrand J, Ziegler A (2010): Percentiles of body mass index of 18-80year-old German adults based on data from the Second National Nutrition Survey. Dtsch Med Wochenschr 135 , 848-852

Hill AJ, Silver EK (1995): Fat, friendless and unhealthy: 9-year old children's perception of body shape stereotypes. Int J Obes Relat Metab Disord 19, 423-430

Hoffmeister U, Bullinger M, Egmond-Frohlich A, Goldapp C, Mann R, Ravens-Sieberer U, Reinehr T, Westenhofer J, Holl RW (2010): Treatment of obesity in pediatric patients in Germany: 
anthropometry, comorbidity and socioeconomic gradients based on the BZgA Observational Study. Klin Padiatr 222, 274-278

Hollingshead AB (2011): Four factor index of social status: working paper. Yale Journal of Sociology $\underline{8}$, 1119

Holm JC, Nowicka P, Farpour-Lambert NJ, O'Malley G, Hassapidou M, Weiss R, Baker JL (2014): The Ethics of Childhood Obesity Treatment - from the Childhood Obesity Task Force (COTF) of European Association for the Study of Obesity (EASO). Obesity Facts $\underline{7}, 274-281$

Hutton B, Fergusson D (2004): Changes in body weight and serum lipid profile in obese patients treated with orlistat in addition to a hypocaloric diet: a systematic review of randomized clinical trials. Am J Clin Nutr 80, 1461-1468

Jaeschke R (2006): Effekte von körperlichem Training bei Kindern und Jugendlichen mit Formen des Asthma bronchiale und, oder der Adipositas: Meta-Analysen empirischer Studien von 1950 bis 2002. Sportwiss. Diss. Köln 2006

Jeffrey RW, Wing RR, Mayer RR (1998): Are smaller weight losses or more achievable weight loss goals better in the long term for obese patients? J Consult Clin Psychol 66, 641-645

Johansson K, Sundstrom J, Marcus C, Hemmingsson E, Neovius M (2014): Risk of symptomatic gallstones and cholecystectomy after a very-low-calorie diet or low-calorie diet in a commercial weight loss program: 1-year matched cohort study. Int J Obes $\underline{38}$, 279-284

Kahlert D, Brand R (2011): Fragebogenerfassung von körperlicher Aktivität. Zeitschr Gesundheitspsychol $19,96-100$

Kersting M, Clausen K (2007): Wie teuer ist eine gesunde Ernährung für Kinder und Jugendliche? Die Lebensmittelkosten der Optimierten Mischkost als Referenz für sozialpolitische Regelleistungen. Ernährungsumschau $\underline{54}, 508-513$

Kirschenbaum DS, Harris ES, Tomarken AJ (1984): Effects of parental involvement in behavioral weight loss therapy for preadolescents Behavior Therapy. 15, 485-500

Kitsantas P, Gallo S, Palla H, Nguyen V, Gaffney K (2016): Nature and nurture in the development of childhood obesity: early infant feeding practices of overweight/obese mothers differ compared to mothers of normal body mass index. J Matern Fetal Neonatal Med 29, 290-293

Kliche T, Mann R (2008): Prevention and care for obese children and adolescents in Germany: Quality and eficiencies of programs and interventions. Bundesgesundheitsblatt - Gesundheitsforschung Gesundheitsschutz $\underline{51}$, 646-656

Kmietowicz Z (2015): Nurture is more important than nature in childhood obesity, study finds. Bmj $\underline{350}$, h817

Knerr I, Keller K, Jaeschke R, Hoffmeister U, Reinehr T, Holl R (2008): Adipositas bei Kindern, Jugendlichen und ihren Eltern. Assoziation des standardisierten BMI zwischen Patienten, Eltern und Geschwistern anhand multizentrischer APV-Daten. Dtsch Med Wochenschr 133, 2448-2453

Korsten-Reck U, Bauer S, Keul J (1994): Sports and nutrition - an out-patient program for adipose children (long-term experience). Int J Sports Med 15, 242-248

Korsten-Reck U, Rudloff C, Kayser R, Eßer K-J, Gruppe M, Emunds U, Kromeyer-Hauschild K, Rücker G, Wolfarth B, Berg A (2002): Freiburger Interventionsprogramm zur ambulanten Therapie der Adipositas im Kindesalter (FITOC). Versicherungsmedizin $\underline{54}$

Korsten-Reck U, Kromeyer-Hauschild K, Wolfarth B, Dickhuth HH, Berg A (2005): Freiburg Intervention Trial for Obese Children (FITOC): results of a clinical observation study. Int J Obes (Lond) 29, 356-361

Kramer MS (1981): Do breast-feeding and delayed introduction of solid foods protect against subsequent obesity? J Pediatr 98, 883-887

Kromeyer-Hauschild K, Wabitsch M, Kunze D, Geller F, Geiß HC, Hesse V, Hippel Av, Jaeger U, Johnsen D, Korte Wea (2001): Perzentile für den Body-mass-Index für das Kindes- und Jugendalter unter Heranziehung verschiedener deutscher Stichproben. Monatsschr Kinderheilkd 807-818

Kromeyer-Hauschild K, Moss A, Wabitsch M (2015): Referenzwerte für den Body-Mass-Index für Kinder, Jugendliche und Erwachsene in Deutschland. Anpassung der AGA-BMI-Referenz im Altersbereich von 15 bis 18 Jahren. Adipositas - Ursachen, Folgeerkrankungen, Therapie. $\underline{9}$, 123-127

Kurth BM (2007): The German Health Interview and Examination Survey for Children and Adolescents (KiGGS): an overview of its planning, implementation and results taking into account aspects of 
quality management. Bundesgesundheitsblatt Gesundheitsforschung Gesundheitsschutz $\underline{50}$, 533546

Kurth BM, Schaffrath Rosario A (2007): The prevalence of overweight and obese children and adolescents living in Germany. Results of the German Health Interview and Examination Survey for Children and Adolescents (KiGGS). Bundesgesundheitsblatt Gesundheitsforschung Gesundheitsschutz $\underline{50}$, 736-743

Levine JA, Eberhardt NL, Jensen MD (1999): Role of nonexercise activity thermogenesis in resistance to fat gain in humans. Science 283, 212-214

Li S, Jin X, Wu S, Jiang F, Yan C, Shen X (2007): The impact of media use on sleep patterns and sleep disorders among school-aged children in China. Sleep 30, 361-367

Linde JA, Jeffery RW, Finch EA, Ng DM, Rothman AJ (2004): Are unrealistic weight loss goals associated with outcomes for overweight women? Obesity Research $\underline{12}$, 569-576

Lindgren G, Strandell A, Cole T, Healy M, Tanner J (1995): Swedish population reference standards for height, weight and body mass index attained at 6 to 16 years (girls) or 19 years (boys). Acta Paediatr $\underline{84}$, 1019-1028

Lioret S, Touvier M, Dubuisson C, Dufour A, Calamassi-Tran G, Lafay L, Volatier JL, Maire B (2009): Trends in child overweight rates and energy intake in France from 1999 to 2007: relationships with socioeconomic status Obesity. (Silver Spring) 17, 1092-1100

Lloyd LJ, Langley-Evans SC, McMullen S (2010): Childhood obesity and adult cardiovascular disease risk: a systematic review. Int J Obes (Lond) $\underline{34}, 18-28$

Lowell BB, Spiegelman BM (2000): Towards a molecular understanding of adaptive thermogenesis. Nature $\underline{404}, 652-660$

MacDonald A, Stunkard A (1990): Body-mass indexes of British separated twins. N Engl J Med $\underline{322} 1530$

Mast M, Kortzinger I, Konig E, Muller MJ (1998): Gender differences in fat mass of 5-7-year old children. Int J Obes Relat Metab Disord 22, 878-884

Matthiessen J, Velsing Groth M, Fagt S, Biltoft-Jensen A, Stockmarr A, Andersen JS, Trolle E (2008): Prevalence and trends in overweight and obesity among children and adolescents in Denmark. Scand J Public Health $\underline{36}$, 153-160

McCarthy HD, Cole TJ, Fry T, Jebb SA, Prentice AM (2006): Body fat reference curves for children. Int J Obes (Lond) 30, 598-602

McLean N, Griffin S, Toney K, Hardeman W (2003): Family involvement in weight control, weight maintenance and weight-loss interventions: a systematic review of randomised trials. Int J Obes Relat Metab Disord 27, 987-1005

McWhorter JW, Wallmann HW, Alpert PT (2003): The obese child: Motivation as a tool for exercise. Journal of Pediatric Health Care 17, 11-17

Mellin LM, Slinkard LA, Irwin CE, Jr. (1987): Adolescent obesity intervention: validation of the SHAPEDOWN program. J Am Diet Assoc 87, 333-338

Molnar D, Torok K, Erhardt E, Jeges S (2000): Safety and efficacy of treatment with an ephedrine/caffeine mixture. The first double-blind placebo-controlled pilot study in adolescents. Int J Obes Relat Metab Disord 24, 1573-1578

Moss A, Wabitsch M, Kromeyer-Hauschild K, Reinehr T, Kurth BM (2007): Prevalence of overweight and adiposity in German school children. Bundesgesundheitsblatt Gesundheitsforschung Gesundheitsschutz $\underline{50}$, 1424-1431

Moss A, Klenk J, Simon K, Thaiss H, Reinehr T, Wabitsch M (2012): Declining prevalence rates for overweight and obesity in German children starting school. Eur J Pediatr 171, 289-299

Mossberg HO (1989): 40-year follow-up of overweight children. Lancet $\underline{2}$, 491-493

Mühlig Y, Wabitsch M, Moss A, Hebebrand J (2014): Gewichtsabnahme bei Kindern und Jugendlichen. Dtsch Arztebl International 111, 818-824

Must A, Strauss RS (1999): Risks and consequences of childhood and adolescent obesity. Int J Obes Relat Metab Disord 23 Suppl 2, S2-11

Neel JV (1962): Diabetes mellitus: a "thrifty" genotype rendered detrimental by "progress"? Am J Hum Genet 14, 353-362

Neumark-Sztainer D, van den Berg P, Hannan PJ, Story M (2006): Self-weighing in adolescents: helpful or harmful? Longitudinal associations with body weight changes and disordered eating. J Adolesc Health $39,811-818$ 
Norgren S, Danielsson P, Jurold R, Lotborn M, Marcus C (2003): Orlistat treatment in obese prepubertal children: a pilot study. Acta Paediatr 92, 666-670

O'Brien PE, Sawyer SM, Laurie C, Brown WA, Skinner S, Veit F, Paul E, Burton PR, McGrice M, Anderson M, et al. (2010): Laparoscopic adjustable gastric banding in severely obese adolescents: a randomized trial. Jama $\underline{303}$, 519-526

Ogden CL, Carroll MD, Flegal KM (2008): High body mass index for age among US children and adolescents, 2003-2006. Jama 299, 2401-2405

Ogden CL, Lamb MM, Carroll MD, Flegal KM (2010): Obesity and socioeconomic status in children and adolescents: United States, 2005-2008. NCHS Data Brief, 1-8

Olds TS, Tomkinson GR, Ferrar KE, Maher CA (2010): Trends in the prevalence of childhood overweight and obesity in Australia between 1985 and 2008. Int J Obes (Lond) 34, 57-66

Olson EA, Visek AJ, McDonnell KA, DiPietro L (2012): Thinness expectations and weight cycling in a sample of middle-aged adults Eating behaviors. $\underline{13}, 142-145$

Oude Luttikhuis H, Baur L, Jansen H, Shrewsbury VA, O'Malley C, Stolk RP, Summerbell CD (2009): Interventions for treating obesity in children. Cochrane Database Syst Rev, Cd001872

Parizkova J, Maffeis C, Poskitt EM: Management through activity. In: Burniat W, Cole TJ, Lissau I, Poskitt EM (Hrsg.): Child and Adolescent Obesity. Causes and consequences, Prevention and Management. Cambridge University Press, Cambridge 2002, 307-326

Petersen C, Schlesinger S (2009): Moby Dick Adipositas-Ursachen, Folgeerkrankungen, Therapie. $\underline{3}, 22-26$

Pettee Gabriel KK, Morrow JR, Jr., Woolsey AL (2012): Framework for physical activity as a complex and multidimensional behavior. J Phys Act Health 9 Suppl 1, S11-18

Pietrobelli A, Faith MS, Allison DB, Gallagher D, Chiumello G, Heymsfield SB (1998): Body mass index as a measure of adiposity among children and adolescents: a validation study. J Pediatr 132, 204-210

Pietrobelli A, Tato L (2005): Body composition measurements: from the past to the future. Acta Paediatr Suppl $\underline{94}, 8-13$

Pinhas-Hamiel O, Dolan LM, Daniels SR, Standiford D, Khoury PR, Zeitler P (1996): Increased incidence of non-insulin-dependent diabetes mellitus among adolescents. J Pediatr $\underline{128}$, 608-615

Polivy J (2001): The false hope syndrome: unrealistic expectations of self-change Int J Obes Rel Metab Disord $\underline{25}$

Poskitt EM (1995): Defining childhood obesity: the relative body mass index (BMI). European Childhood Obesity group. Acta Paediatr $\underline{84}, 961-963$

Reinehr T, Wabitsch M (2003): Strukturierte Erfassung der Therapieangebote für adipöse Kinder und Jugendliche. Monatsschr Kinderheilkd 151, 757-761

Reinehr T, Brylak K, Alexy U, Kersting M, Andler W (2003): Predictors to success in outpatient training in obese children and adolescents. Int J Obes Relat Metab Disord 27, 1087-1092

Reinehr T, Wabitsch M, Andler W, Beyer P, Bottner A, Chen-Stute A, Fromme C, Hampel O, Keller KM, Kilian U, et al. (2004): Medical care of obese children and adolescents. APV: a standardised multicentre documentation derived to study initial presentation and cardiovascular risk factors in patients transferred to specialised treatment institutions. Eur J Pediatr $\underline{163}, 308-312$

Reinehr T, Andler W, Denzer C, Siegried W, Mayer H, Wabitsch M (2005): Cardiovascular risk factors in overweight German children and adolescents: relation to gender, age and degree of overweight. Nutr Metab Cardiovasc Dis $\underline{15}$, 181-187

Reinehr T, de Sousa G, Wabitsch M (2006): Changes of cardiovascular risk factors in obese children effects of inpatient and outpatient interventions. J Pediatr Gastroenterol Nutr 43 , 506-511

Reinehr T, Kiess W, De Sousa G, Stoffel-Wagner B, Wunsch R (2006): Intima media thickness in childhood obesity: relations to inflammatory marker, glucose metabolism, and blood pressure Metabolism. $\underline{55}, 113-118$

Reinehr T, Hinney A, de Sousa G, Austrup F, Hebebrand J, Andler W (2007): Definable somatic disorders in overweight children and adolescents. J Pediatr 150, 618-622, 622.e611-615

Reinehr T, Temmesfeld M, Kersting M, de Sousa G, Toschke AM (2007): Four-year follow-up of children and adolescents participating in an obesity intervention program. Int J Obes (Lond) $\underline{31}$, 10741077

Reinehr T, Widhalm K, l'Allemand D, Wiegand S, Wabitsch M, Holl RW (2009): Two-year follow-up in 21,784 overweight children and adolescents with lifestyle intervention. Obesity (Silver Spring) 17, 1196-1199 
Reinehr T, Kleber M, Lass N, Toschke AM (2010): Body mass index patterns over 5 y in obese children motivated to participate in a 1-y lifestyle intervention: age as a predictor of long-term success. Am J Clin Nutr 91, 1165-1171

Röbl M, Knerr I, Keller KM, Jaeschke R, Hoffmeister U, Reinehr T, Holl RW (2008): Adipositas bei Kindern, Jugendlichen und ihren Eltern: Assoziation des standardisierten BMI zwischen Patienten, Eltern und Geschwistern anhand multizentrischer APV-Daten. Deutsche Medizinische Wochenschrift $133,2448-2453$

Röbl M, de Souza M, Schiel R, Gellhaus I, Zwiauer K, Holl RW, Wiegand S (2013): The key role of psychosocial risk on therapeutic outcome in obese children and adolescents. Results from a longitudinal multicenter study. Obes Facts $\underline{6}$, 297-305

Rolland-Cachera MF (2011): Childhood obesity: current definitions and recommendations for their use. Int J Pediatr Obes 6 , 325-331

Rolland-Cachera MF, Cole TJ, Sempe M, Tichet J, Rossignol C, Charraud A (1991): Body Mass Index variations: centiles from birth to 87 years. Eur J Clin Nutr $\underline{45}$, 13-21

Rosario AS, Kurth BM, Stolzenberg H, Ellert U, Neuhauser H (2010): Body mass index percentiles for children and adolescents in Germany based on a nationally representative sample (KiGGS 20032006). Eur J Clin Nutr $\underline{64}$, 341-349

Roth C, Lakomek M, Müller H, Harz KJ (2002): Adipositas im Kindesalter - Ursachen und Therapiemöglichkeiten. Monatsschr Kinderheilkd 150, 329-336

Saris WH, Blair SN, van Baak MA, Eaton SB, Davies PS, Di Pietro L, Fogelholm M, Rissanen A, Schoeller D, Swinburn B, et al. (2003): How much physical activity is enough to prevent unhealthy weight gain? Outcome of the IASO 1st Stock Conference and consensus statement. Obes Rev $\underline{4}, 101-114$

Schenk L, Ellert U, Neuhauser H (2007): Kinder und Jugendliche mit Migrationshintergrund in

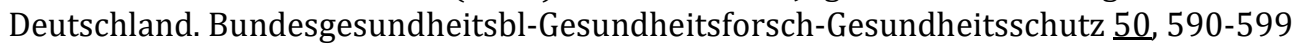

Sekine M, Yamagami T, Handa K, Saito T, Nanri S, Kawaminami K, Tokui N, Yoshida K, Kagamimori S (2002): A dose-response relationship between short sleeping hours and childhood obesity: results of the Toyama Birth Cohort Study. Child Care Health Dev 28, 163-170

Sherry B, Jefferds ME, Grummer-Strawn LM (2007): Accuracy of adolescent self-report of height and weight in assessing overweight status: a literature review. Arch Pediatr Adolesc Med 161, 11541161

Siegfried W, Kromeyer-Hauschild K, Zabel G, Siegfried A, Wabitsch M, Holl RW (2006): Long-term inpatient treatment of extreme juvenile obesity: an 18-month catamnestic study. MMW Fortschr Med 148, 39-41

Skelton JA, Beech B (2011): Attrition in paediatric weight management: a review of the literature and new directions. Obesity Reviews $\underline{12}$, e273-e281

Skelton JA, Irby MB, Grzywacz JG, Miller G (2011): Etiologies of obesity in children: nature and nurture. Pediatr Clin North Am $\underline{58}$, 1333-1354, ix

Spruijt-Metz D (2011): Etiology, Treatment and Prevention of Obesity in Childhood and Adolescence: A Decade in Review. J Res Adolesc 21, 129-152

Spyckerelle Y, Gueguen R, Guillemot M, Tosi E, Deschamps JP (1988): Adiposity indices and clinical opinion. Ann Hum Biol $\underline{15}$ 45-54

Srinivasan S, Ambler GR, Baur LA, Garnett SP, Tepsa M, Yap F, Ward GM, Cowell CT (2006): Randomized, controlled trial of metformin for obesity and insulin resistance in children and adolescents: improvement in body composition and fasting insulin. J Clin Endocrinol Metab 91, 2074-2080

Stachow R, Stübing $K$, van Egmond-Fröhlich A: Trainermanual leichter, aktiver, gesünder: interdisziplinäres Konzept für die Schulung übergewichtiger oder adipöser Kinder und Jugendlicher. Aid-Infodienst Verbraucherschutz, Ernährung, Landwirtschaft, Bonn 2004

Staffieri JR (1967): A study of social stereotype of body image in children. J Pers Soc Psychol $\underline{7}, 101-104$

Story M, Kaphingst KM, Robinson-O'Brien R, Glanz K (2008): Creating healthy food and eating environments: policy and environmental approaches. Annu Rev Public Health $\underline{29}$, 253-272

Strauss R (1999): Childhood obesity. Curr Probl Pediatr 29, 1-29

Sugerman HJ, Londrey GL, Kellum JM, Wolf L, Liszka T, Engle KM, Birkenhauer R, Starkey JV (1989): Weight loss with vertical banded gastroplasty and Roux-Y gastric bypass for morbid obesity with selective versus random assignment. Am J Surg 157, 93-102 
Sundblom E, Petzold M, Rasmussen F, Callmer E, Lissner L (2008): Childhood overweight and obesity prevalences levelling off in Stockholm but socioeconomic differences persist. Int J Obes (Lond) $\underline{32}$, 1525-1530

Tambalis KD, Panagiotakos DB, Kavouras SA, Kallistratos AA, Moraiti IP, Douvis SJ, Toutouzas PK, Sidossis LS (2010): Eleven-year prevalence trends of obesity in Greek children: first evidence that prevalence of obesity is leveling off. Obesity (Silver Spring) 18, 161-166

Telch CF, Agras WS (1993): The effects of a very low calorie diet on binge eating. Behavior Therapy $\underline{24}$, 177-193

Togashi K, Masuda H, Rankinen T, Tanaka S, Bouchard C, Kamiya H (2002): A 12-year follow-up study of treated obese children in Japan Int J Obes Relat Metab Disord 26, 770-777

Toschke AM, Ludde R, Eisele R, von Kries R (2005): The obesity epidemic in young men is not confined to low social classes--a time series of 18-year-old German men at medical examination for military service with different educational attainment. Int J Obes $\underline{29}, 875-877$

van der Horst K, Oenema A, Ferreira I, Wendel-Vos W, Giskes K, van Lenthe F, Brug J (2007): A systematic review of environmental correlates of obesity-related dietary behaviors in youth. Health Educ Res 22, 203-226

Vanhala M, Vanhala P, Kumpusalo E, Halonen P, Takala J (1998): Relation between obesity from childhood to adulthood and the metabolic syndrome: population based study. Bmj $\underline{317} 319$

Vanwormer JJ, French SA, Pereira MA, Welsh EM (2008): The impact of regular self-weighing on weight management: a systematic literature review. Int J Behav Nutr Phys Act $\underline{5}, 54$

Wabitsch M (2000): Overweight and obesity in European children: definition and diagnostic procedures, risk factors and consequences for later health outcome. Eur J Pediatr 159 Suppl 1, 8-13

Wabitsch M, Hauner H, Heinze E, Muche R, Bockmann A, Parthon W, Mayer H, Teller W (1994): Body-fat distribution and changes in the atherogenic risk-factor profile in obese adolescent girls during weight reduction. Am J Clin Nutr 60, 54-60

Wabitsch M, Kiess W, Neef M, Reinehr T: Adipositas bei Kindern und Jugendlichen. In: Hauner A, Wirth H (Hrsg.): Adipositas. Ätiologie, Folgekrankheiten, Diagnostik, Therapie. 4. Auflage; Springer, Berlin Heidelberg 2013, 367 - 388

Westenhoefer J (2001): The therapeutic challenge: behavioral changes for long-term weight maintenance. Int J Obes Relat Metab Disord 25, 85-88

Whitaker RC, Wright JA, Pepe MS, Seidel KD, Dietz WH (1997): Predicting obesity in young adulthood from childhood and parental obesity. N Engl J Med 337, 869-873

Whitaker RC, Pepe MS, Wright JA, Seidel KD, Dietz WH (1998): Early adiposity rebound and the risk of adult obesity. Pediatrics $\underline{101}$, E5

Whitman SC, Miller DB, Wolfe BM, Hegele RA, Huff MW (1997): Uptake of type III hypertriglyceridemic VLDL by macrophages is enhanced by oxidation, especially after remnant formation. Arterioscler Thromb Vasc Biol 17, 1707-1715

WHO (2000): Obesity: preventing and managing the global epidemic. Report of a WHO consultation. World Health Organ Tech Rep Ser 894, i-xii, 1-253

WHO (2006): WHO Child Growth Standards based on length/height, weight and age. Acta Paediatr Suppl $\underline{450}, 76-85$

Wiegand S, Dannemann A, Vahabzadeh Z, Ernst M, Krude H, Grüters A (2005): Who needs what? New approaches to multidisciplinary diagnostics and therapy for adipose children and youths in a multiethnic city. Bundesgesundheitsblatt Gesundheitsforsch.Gesundheitsschutz 48, 307-314

Wiegand S, Keller KM, Lob-Corzilius T, Pott W, Reinehr T, Röbl M, Stachow R, Tuschy S, Weidanz I, Widhalm K, et al. (2014): Predicting weight loss and maintenance in overweight/obese pediatric patients. Horm Res Paediatr $\underline{82}$, 380-387

Wilfley DE, Stein RI, Saelens BE, al. e (2007): Efficacy of maintenance treatment approaches for childhood overweight: a randomized controlled trial. JAMA 298, 1661-1673

Winkler J, Stolzenberg H (1999): Der Sozialschichtindex im Bundes-Gesundheitssurvey. Gesundheitswesen 61, 178-S183

Yin TJ, Wu FL, Liu YL, Yu S (2005): Effects of a weight-loss program for obese children: a "mix of attributes" approach. J Nurs Res $\underline{13}, 21-30$

Yoo JH, Lee EJ, Kwak CK, Sohn EH, Koh BH, Song IB, Lee KS (2005): Clinical trial of herbal formula on weight loss in obese Korean children. Am J Chin Med 33, 713-722 
Zwiauer KF, Howanietz H, Steger H, Widhalm K (1989): Glucose tolerance test in morbidly obese children and adolescents. Klin Padiatr 201, 118-122

Zwiauer KF, Pakosta R, Mueller T, Widhalm K (1992): Cardiovascular risk factors in obese children in relation to weight and body fat distribution. J Am Coll Nutr 11 Suppl, 41s-50s

\section{Internetquellen}

AGA: Informationen und Stellungnahme zu bariatrisch-chirurgischen Maßnahmen bei Jugendlichen mit extremer Adipositas der Arbeitsgemeinschaft Adipositas im Kindes- und Jugendalter (AGA) (2012) unter:

http://aga.adipositasgesellschaft.de/fileadmin/PDF/Adipositas/XXL_Stellungnahme zu bariatrischchirurgischen Eingriffen bei Jugendlichen finale Version AGA Vorstand.pdf: Zugriff am 11.02.2016

AGA: Komorbidität (2011) unter: http://www.aga.adipositas-gesellschaft.de/index.php?id=321: Zugriff am 12.02.2016

AGA: Konsensbasierte (S2) Leitlinie zur Diagnostik, Therapie und Prävention von Übergewicht und Adipositas im Kindes- und Jugendalter (2015) unter: http://www.adipositasgesellschaft.de/fileadmin/PDF/Leitlinien/AGA S2 Leitlinie.pdf: Zugriff am 07.01.2018

AGA: Konsensuspapier - Patientenschulungsprogramme für Kinder und Jugendliche mit Adipositas (2004) unter: http://www.aga.adipositasgesellschaft.de/fileadmin/PDF/daten/Konsensuspapier Patientenschulung.pdf: Zugriff am 07.01.2018

AGA: Therapie der Adipositas im Kindes- und Jugendalter - Evidenzbasierte Leitlinie der Arbeitsgemeinschaft Adipositas im Kindes- und Jugendalter (AGA) und der beteiligten medizinischen-wissenschaftlichen Fachgesellschaften, Berufsverbände und weiterer Organisationen (2009) unter: http://www.aga.adipositasgesellschaft.de/fileadmin/PDF/Leitlinien/Leitlinie-AGA-S3-2009.pdf: Zugriff am 07.01.2018

AGA: Zertifizierung von Therapieeinrichtungen mit Patientenschulungsprogrammen für adipöse Kinder und Jugendliche durch die AGA / DAG (2008) unter: http://www.aga.adipositasgesellschaft.de/fileadmin/PDF/daten/Zertifizierung.pdf: Zugriff am 04.04.2016

BABELUGA: Das BABELUGA-Baustellen-Material (o.J.) unter: $\underline{\text { http://www.babeluga- }}$ berlin.de/adipositas/index.php/babeluga-baustellen-material-i: Zugriff am 03.05.2016

BZgA: Bericht zur EvAKuJ-Studie im Rahmen des Qualitätssicherungsprozesses der BZgA zur Prävention und Therapie von Übergewicht bei Kindern und Jugendlichen (2011) unter: https://www.bzgakinderuebergewicht.de/fileadmin/user upload/pdf/fachkraefte/Abschlussbericht Beobachtungs studie 04102012.pdf: Zugriff am 07.01.2018 
IOTF: International Obesity Task Force EU Platform Briefing Paper (2005) unter: http://static.publico.pt/docs/pesoemedida/EU Platform Diet PA Health 2005.pdf: Zugriff am 07.01 .2018

KgAS: Schulungszentren (2011) unter: http://www.adipositas-schulung.de/schulungszentren.php: Zugriff am 03.05.2016

RKI: Referenzperzentile für anthropometrische Maßzahlen und Blutdruck aus der Studie zur Gesundheit von Kindern und Jugendlichen in Deutschland (KiGGS) (2013) unter: https://www.rki.de/DE/Content/Gesundheitsmonitoring/Gesundheitsberichterstattung/GBEDo wnloadsB/KiGGS Referenzperzentile.pdf? blob=publicationFile: Zugriff am 07.01.2018

WHO: $\quad$ Growth reference 5 - 19 years (2007) unter: http://www.who.int/growthref/who2007 bmi for age/en/: Zugriff am 04.02.2016

WHO: The WHO Child Growth Standards: BMI-for-age, simplified field tabels (o.J.) unter: http://www.who.int/childgrowth/standards/bmi for age field/en/: Zugriff am 04.02.2016 


\section{Danksagung}

An dieser Stelle möchte ich mich bei Frau Prof. Dr. med. Jutta Gärtner für die Möglichkeit zur Promotion in der Abteilung für Kinder- und Jugendmedizin bedanken.

Weiterhin danke ich Dr. med. Markus Röbl für die sehr gute Betreuung und die Zeit, die er sich stets für meine Fragen genommen hat.

Nicht zuletzt gilt mein besonderer Dank meinen Geschwistern, insbesondere meinem Bruder Yassin, für die unermüdliche Hilfestellung und Unterstützung bei der statistischen Auswertung. 